\title{
Machine Learning Techniques for Program Representation and Comprehension with Applications to Mobile Security
}

\author{
Narayanan Annamalai
}

School of Electrical and Electronic Engineering

A thesis submitted to the Nanyang Technological University in partial fulfillment of the requirement for the degree of

Doctor of Philosophy 



\section{Acknowledgments}

This dissertation would not have been possible without the support of many people who in one way or another have contributed and extended their precious knowledge and experience in my $\mathrm{PhD}$ studies. It is my pleasure to express my gratitude to them.

First of all, I would like to express my gratitude to my Ph.D. advisor, Assoc. Prof. Dr. Chen Lihui, for her advice, constant support and encouragement. Second, I am equally grateful to Asst. Prof. Dr. Yang Liu for his guidance and support throughout my Ph.D. study. It is their guidance and timely help that took me towards the completion of this thesis. They have always been appreciative of new (often unconventional) ideas and encouraged critical thinking and technical writing, which have helped me improve my skills.

Third, I am grateful to Prof. Tan Hee Beng Kuan and Prof. Cong Gao, who were in my Thesis Advisory Committee, for their valuable suggestions and comments on my work.

I would like to thank Anand Vetrivel and Mahinthan Chandramohan, who have contributed immensely with their suggestions to my research. Without them, the works in this thesis would not have been possible. I am grateful to Rajasekar Venkatesan, Meng Guozhu, Dr. Yinxing Xue, Dr. Sathya Narayanan Sekar, Dr. Deepan Balakrishnan and Prabhash Kumarasinghe for their valuable suggestions and brainstorming sessions that helped me to improve my work.

I am thankful to the School of Electrical and Electronics Engineering, Nanyang Technological University (NTU) for providing me the research student scholarship. I would like to thank the Media Technology Laboratory executive, Mrs. Leow How for her support (especially for granting me access to servers \& workstations!).

I would like to thank my friends Ajith, Karthik Narayanan, Sridhar and Balasubramanian for the all the good times and memories that they have offered me over the years.

Last but not least, I wish to express my utmost gratitude to my mother and sister for their unconditional support, understanding and trust. 


\section{Contents}

Acknowledgments

Contents ii

List of Figures $\quad$ v

List of Tables vii

$\begin{array}{ll}\text { List of Abbreviations } & \text { ix }\end{array}$

List of General Notations $\quad$ xi

Summary xiii

1 Introduction $\quad 1$

1.1 Motivations and Goals . . . . . . . . . . . . . . . . 3

1.2 Main Works and Contributions . . . . . . . . . . . . . 6

1.3 Thesis Outline . . . . . . . . . . . . . . . . . 12

2 Background and Preliminaries $\quad \mathbf{1 4}$

2.1 Background on Android Security and Defense . . . . . . . . . . . . . . . 14

2.1 .1 Android System . . . . . . . . . . . . . . . . . . . 14

2.1.2 Android Malware Detection . . . . . . . . . . . . . . 15

2.1.3 Android Clone Detection . . . . . . . . . . . . . . . . . . 17

2.1.4 Defense: Existing Techniques and Analysis Paradigms . . . . . . . 19

2.2 Machine Learning Preliminaries . . . . . . . . . . . . . . . . . 20

2.2.1 Kernel Methods and Graph Kernels . . . . . . . . . . . . . . . 20

2.2.2 Multiple Kernel Learning . . . . . . . . . . . . . . . . . 21

2.2 .3 Online Learning . . . . . . . . . . . . . . . . . . . . . . . . . . . 23

2.2.4 Representation (Deep) Learning . . . . . . . . . . . . . . . . 27

3 Contextual Weisfeiler-Lehman Kernel for Malware Detection 29

3.1 Introduction . . . . . . . . . . . . . . . . . . . . 29

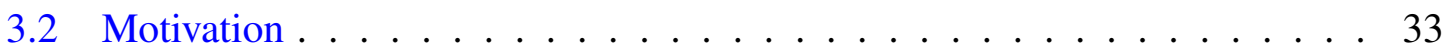

3.3 Definitions and Notations . . . . . . . . . . . . . . 35 
3.4 Contextual Weisfeiler-Lehman Graph Kernel . . . . . . . . . . . . . . 36

3.4.1 Regular Weisfeiler-Lehman Graph Kernel . . . . . . . . . . . . . . 36

3.4.2 Contextual Weisfeiler-Lehman Graph Kernel . . . . . . . . . . . . 37

3.5 Evaluation . . . . . . . . . . . . . . . . . . 42

3.5 .1 Datasets ........................ 43

3.5.2 Implementation \& Comparative Analysis . . . . . . . . . . . . 44

3.5.3 Experimental Design . . . . . . . . . . . . . . . . . . 45

3.5.4 Results and Discussions . . . . . . . . . . . . . . . 47

3.6 Related Work . . . . . . . . . . . . . . . . . . . . 53

3.6.1 General Purpose Graph Kernels . . . . . . . . . . . . . . . . . 54

3.6.2 Application-specific Graph Kernels . . . . . . . . . . . . . 55

3.7 Limitations . . . . . . . . . . . . . . . . . . . . . 55

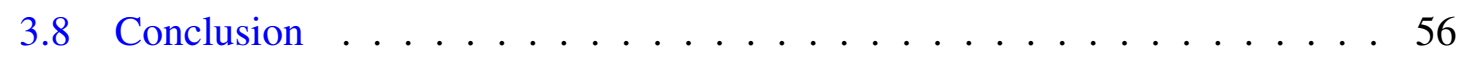

4 Context-aware, Adaptive and Scalable Android Malware Detection through Online Learning $\quad 57$

4.1 Introduction . . . . . . . . . . . . . . . . . 57

4.1.1 Malware Detection using Graph Representations . . . . . . . . . 58

4.1.2 Challenges in ML based Malware Detection . . . . . . . . . . . . 59

4.1 .3 Our Approach . . . . . . . . . . . . . . . . . 6 61

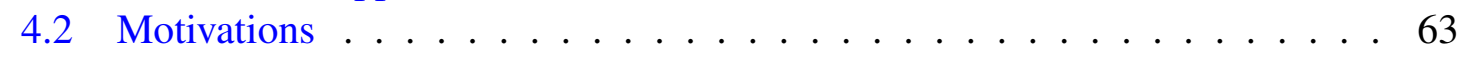

4.2.1 Handling Population Drift . . . . . . . . . . . . . . 63

4.2.2 Handling Large High-dimensional Data . . . . . . . . . . . . . . . . . 64

4.2.3 Handling Real-time Streaming . . . . . . . . . . . . . . . . . 65

4.3 Methodology . . . . . . . . . . . . . . . . 65

4.3.1 CaSANDRA: Framework overview . . . . . . . . . . . 66

4.3.2 Static Analysis . . . . . . . . . . . . . . . . 67

4.3.3 Feature Extraction \& Representation using CWLK . . . . . . . . . 70

4.3 .4 Online Learning . . . . . . . . . . . . . . . . . . . . 72

4.3.5 Explaining CaSANDRA's predictions . . . . . . . . . . . . 75

4.4 Experimental Design \& Implementation . . . . . . . . . . . . . . . . . . 76

4.4.1 Research Questions . . . . . . . . . . . . . . . 76

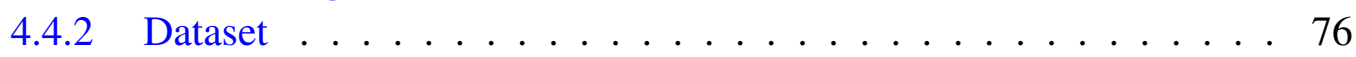

4.4 .3 Experimental Setup . . . . . . . . . . . . . . . . 78

4.4.4 Implementation and Comparative Analysis . . . . . . . . . . 78

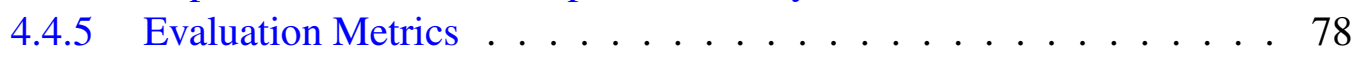

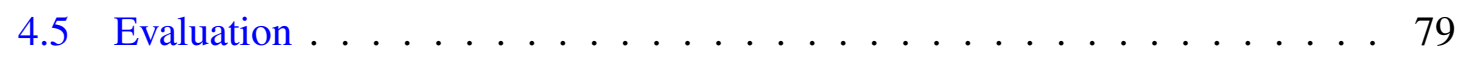

4.5.1 RQ1: Accuracy . . . . . . . . . . . . . . . 79

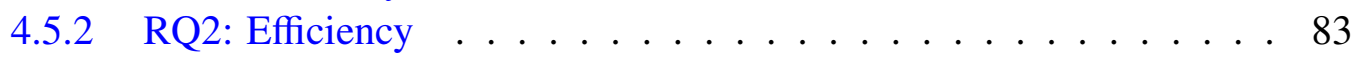

4.5.3 RQ3: Explainablity . . . . . . . . . . . . . . 85

4.5.4 RQ4: Adaptiveness . . . . . . . . . . . . . . . . . . . . . . . . . . . . . . . . . .

4.6 Related Work . . . . . . . . . . . . . . . . . . . 92

4.7 Limitations . . . . . . . . . . . . . . . . . . . . . . . 94 
5 A Multi-view Context-aware Approach to Android Malware Detection and Malicious Code Localization $\quad 96$

5.1 Introduction . . . . . . . . . . . . . . . . . . . 96

5.1.1 Challenges in Single-perspective Malware Detection . . . . . . . 97

5.1 .2 Our Approach . . . . . . . . . . . . . . . . . . . . 989

5.2 Motivations . . . . . . . . . . . . . . . . . . . 101

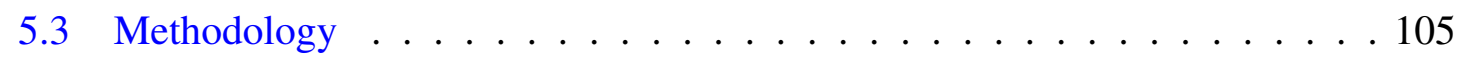

5.3.1 MKLDROID - Framework Overview . . . . . . . . . . . . . 105

5.3 .2 Static Analysis . . . . . . . . . . . . . . . . . 107

5.3.3 Feature Extraction, Selection and Representation . . . . . . . . 110

5.3.4 Multiple Kernel Learning . . . . . . . . . . . . . . . . . . . . . 111

5.3.5 Malicious Code Localization . . . . . . . . . . . . . . . . . . . 114

5.4 Experimental Design and Implementation . . . . . . . . . . . . . . 120

5.4.1 Research Questions . . . . . . . . . . . . . . . . . 120

5.4 .2 Datasets \& Experiments . . . . . . . . . . . . . . . . 121

5.4 .3 Experimental Setup . . . . . . . . . . . . . . . . 126

5.4.4 Implementation and Comparative Analysis . . . . . . . . . . . 126

5.4.5 Evaluation Metrics . . . . . . . . . . . . . . . . . 127

5.5 Results and Discussions . . . . . . . . . . . . . . . . . . 127

5.5.1 RQ1: Accuracy . . . . . . . . . . . . . . . . 127

5.5 .2 RQ2: Efficiency . . . . . . . . . . . . . . . . . 137

5.5.3 RQ3: Locating Malice Code . . . . . . . . . . . . . . . . . . 141

5.6 Related Work . . . . . . . . . . . . . . . . . . . . . . . 153

5.6.1 PRG based Android Malware Detection . . . . . . . . . . . . . 153

5.6.2 Multi-perspective Approaches . . . . . . . . . . . . . . . . 154

5.6 .3 Malicious Code Localization . . . . . . . . . . . . . . . . . . . . . . . . . . . . . . . . . . . . . . .

5.7 Limitations . . . . . . . . . . . . . . . . . . . . . . . 157

5.8 Conclusions . . . . . . . . . . . . . . . . . . . . 158

6 Subgraph2vec: Learning Distributed Representations of Rooted Sub-graphs $\begin{array}{lr}\text { from Large Graphs } & 160\end{array}$

6.1 Introduction . . . . . . . . . . . . . . . . . . . . . . . 160

6.1 .1 Graph Kernels . . . . . . . . . . . . . . . . . . 161

6.1.2 Limitations of Existing Graph Kernels . . . . . . . . . . . . . . . 163

6.1.3 Existing Solution: Deep Graph Kernels . . . . . . . . . . . . . . 164

6.1 .4 Our Approach . . . . . . . . . . . . . . . . . . . . . 166

6.2 Related Work . . . . . . . . . . . . . . . . . . . . . 167

6.3 Problem Statement . . . . . . . . . . . . . . . . . . . 168

6.4 Background: Language Models . . . . . . . . . . . . . . . . . . . . . . . . . . . . . . . . . . . . . . . . . . .

6.4.1 Skip Gram . . . . . . . . . . . . . . . . . . 170

6.4 .2 Negative Sampling . . . . . . . . . . . . . . . . . . . . 171

6.5 Method: Learning Sub-graph Representations . . . . . . . . . . . . . . 172 
6.5 .1 Overview ............................ 172

6.5 .2 Algorithm: SubGRAPH2VEC . . . . . . . . . . . . . 172

6.5.3 Relation to Deep WL kernel . . . . . . . . . . . . . . . . . . 177

6.5 .4 Use Cases . . . . . . . . . . . . . . . . . . . . . . . 177

6.6 Evaluation . . . . . . . . . . . . . . . . . . 178

6.6.1 Classification on Benchmark Datasets . . . . . . . . . . . . . 179

6.6 .2 Clone Detection . . . . . . . . . . . . . . . . 181

6.6 .3 Malware Detection . . . . . . . . . . . . . . 183

6.7 Conclusion . . . . . . . . . . . . . . . . 185

7 Conclusion and Future Research 186

7.1 Summary of Completed Work . . . . . . . . . . . . . . . . 186

7.2 Future Work . . . . . . . . . . . . . . . . . 188

$\begin{array}{ll}\text { Author's Publications } & 191\end{array}$

$\begin{array}{lr}\text { References } & 193\end{array}$ 


\section{List of Figures}

1.1 Overview of the thesis . . . . . . . . . . . . 7

1.2 Road-map of contribution chapters in this dissertation . . . . . . . . . 12

2.1 Android OS Architecture . . . . . . . . . . . . . . . . 15

3.1 Location information being leaked in Geinimi (malware) and Yahoo Weather (benign) apps. (a) code snippet corresponding to leaking location information in both the apps. (b) DDG corresponding to the location leak. (c) Geinimi's DDG illustrating that it leaks information without the user's knowledge. (d) Yahoo Weather's DDG illustrating that it leaks information with the user's knowledge. . . . . . . . . . . . . . . . . 33

3.2 WLK and CWLK neighborhood labels for the node getLatitude from Geinimi and Yahoo Weather apps. . . . . . . . . . . . . . . . 40

3.3 No. of features obtained for various heights of CWLK. . . . . . . . . . 46

3.4 Training and Testing durations for different values of $h$ on ICFG representation (avg. over 5 runs) . . . . . . . . . . . . . 50 50

3.5 Comparison on Efficiency of CWLK with state-of-the-art malware detectors $($ avg. over 5 runs $) \ldots \ldots . \ldots . \ldots 52$

4.1 Casandra: Framework overview . . . . . . . . . . . . . . 66

4.2 Timeline based distribution of apps in our large-scale ITW dataset (with malware and benign apps proportions). . . . . . . . . . . . 78

4.3 Cumulative number of features observed over time for our ITW dataset. . . 80

4.4 Casandra: : Cumulative error rates for Casandra vs. batch learning algorithms (a) CASANDRA Vs. four variants of SVM, (b) CASANDRA Vs. four variants of Drebin, (c) CASANdRa Vs. four variants of CSBD. . . . . . . . 82

4.5 Casandra Vs. Drebin: Comparing explainability on three popular families from the DR dataset . . . . . . . . . . . . . . . . 887

4.6 Illustration of how CASANDRA adapts to the malware evolution and population drift in the DR dataset. The $\mathrm{X}$ axis shows the distribution of familyspecific malware samples. Y axis (left) shows the number of samples and Y axis (right) shows the weights of individual features. The legend contains CWLK subgraph features (format: $<$ feature $\mathrm{id}>$ : $<$ context $>\{<$ root-node $>$ $+<$ neighbor-list $>\}) \ldots \ldots \ldots \ldots$. . . . . . . . . . 89 
5.1 Extracting different five semantic perspectives of the Geinimi variant. (a) code snippet corresponding to leaking location, IMEI and contacts. (b) ICFG representation of the code. (c) Contextual API Dependency Graph corresponding to the code snippet. (d) Contextual Permission Dependency Graph. (e) Contextual Source \& Sink Dependency Graph. (f) Contextual ICFG with Dalvik bytecode instructions used as node labels. (g) Contextual ICFG with textual CFG signatures used as node labels. . . . . . . . . 102

5.2 MKLDroID - Overview . . . . . . . . . . . . . . . . . . . . . 105

5.3 Qualitative Analysis: Comparison of base kernels and kernel combinations through visualizing kernel matrices as heatmaps . . . . . . . . . . 136

5.4 Depictions of CICFG of the ADRD sample from five different perspectives. (a) API view: the CICFG nodes are assigned m-scores according to their CADG features i.e., API m-score (b) Permission view (c) Src-sink view (d) Instruction view (e) Signature view (f) MKL view: for every CICFG node, the m-scores assigned are MKL based linear combinations of their individual views' m-scores. . . . . . . . . . . . . . . . . . . . . . 142

5.5 Depictions of CICFG of the Geinimi sample from five different perspectives. (a) API view: the ICFG nodes are assigned m-scores according to their CADG features i.e., API m-score (b) Permission view (c) Src-sink view (d) Instruction view (e) Signature view (f) MKL view:for every CICFG node, the m-scores assigned are MKL based linear combinations of their individual views' m-scores. . . . . . . . . . . . . . . . . . . . . 148

6.1 Dependency schema of a set of rooted subgraphs of degree 1 ((a), (d)), 2 ((b)) and 3 ((c)) in an Android malware's API dependency graph. The root nodes are marked with a star. Graph (b) can be derived from (a) by adding a node and an edge. Graph (c) can be derived from (b) in a similar fashion. Graph (d) is highly dissimilar from all the other graphs and is not readily derivable from any of them. . . . . . . . . . . . . . . . 162

6.2 Deep WL kernel Vs Subgraph2vecPre-training Durations. . . . . . . . . . 181 


\section{List of Tables}

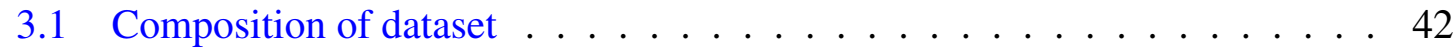

3.2 Dataset Statistics . . . . . . . . . . . . . . . . . . . . 43

3.3 Comparison of CWLK against state-of-the-art Graph Kernels (metrics expressed as $\%$ values) - avg. over 5 runs . . . . . . . . . . . . . 48

3.4 Comparison of CWLK based malware detection against the state-of-the-art detectors (avg. over 5 runs) . . . . . . . . . . . . . . . 51

4.1 In-the-wild Dataset with apps dated from Jan 12014 to Aug 122014 . . . 77

4.2 Comparing CASANDRA's efficiency against that state-of-the-art methods in terms of training and testing durations $\ldots \ldots \ldots \ldots$. . . . . 84

5.1 Datasets used in evaluation . . . . . . . . . . . . . . . . . 122

5.2 List of experiments conducted and the composition of training and test sets 123

5.3 CE1 Results: Precision, Recall \& F-measure (avg \pm std.) . . . . . . . . 128

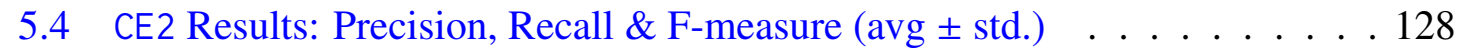

5.5 CE3 Results: Precision, Recall and F-measure . . . . . . . . . . . . . . 131

5.6 WEx Results: Precision, Recall and F-measure . . . . . . . . . . . 133

5.7 Weights of MKLDroID's base kernels - learnt using SMO-MKL [92] . . . 134

5.8 CE1 and CE2 Efficiency: Average training and testing durations . . . . . 138

5.9 MCLEx: Quantitative Evaluation of malicious code localization on the MYST dataset . . . . . . . . . . . . . . . . . . . 151

6.1 Representation Learning from Graphs . . . . . . . . . . . . . . . 168

6.2 Benchmark dataset statistics . . . . . . . . . . . . . . . . . . . 179

6.3 Average Accuracy ( \pm std dev.) for SubGRAPH2 vec and state-of-the-art graph kernels on benchmark graph classification datasets . . . . . . . . . 179

6.4 Clone Detection Dataset Statistics _. . . . . . . . . . . . . . . . 181

6.5 Clone Detection - Results . . . . . . . . . . . . . . . . . . . . . . . . . 182

6.6 Malware Detection Dataset Statistics . . . . . . . . . . . . . . . . . . 183

6.7 Malware Detection - Results . . . . . . . . . . . . . . . . . . . 184 


\section{List of Abbreviations}

\begin{tabular}{|c|c|}
\hline ADG & API Dependency Graph \\
\hline API & Application Programming Interface \\
\hline $\mathbf{A P}$ & Affinity Propagation \\
\hline ARI & Adjusted Rand Index \\
\hline BoF & Bag-of-Features \\
\hline CADG & Contextual API Dependency Graph \\
\hline CFG & Control Flow Graph \\
\hline CG & Call Graph \\
\hline CICFG & Contextual Inter-procedural Control Flow Graph \\
\hline CPDG & Contextual Permission Dependency Graph \\
\hline CSBD & CFG Signature Based Detection \\
\hline CSSDG & Contextual Source and sink Dependency Graph \\
\hline CWLK & Contextual Weisfeiler-Lehman Graph Kernel \\
\hline $\mathbf{C \& C}$ & Command and Control \\
\hline DGK & Deep Graph Kernel \\
\hline DKF & DroidKungFu malware \\
\hline FNR & False Negative Rate \\
\hline FPR & False Positive Rate \\
\hline ICFG & Inter-procedural Control Flow Graph \\
\hline IG & Information Gain \\
\hline ITW & In-the-wild \\
\hline
\end{tabular}


LR Logistic Regression classifier

MKL Multiple Kernel Learning

ML Machine Learning

NHGK Neighborhood Hashing Graph Kernel

NSPDK Neighborhood Subgraph Pairwise Distance kernel

OS Operating System

PDG Program Dependency Graph

PRG Program Representation Graph

RF Random Forest

RKHS Reproducing Kernel Hilbert space

SGD Stochastic Gradient Descent

SMO Sequential Minimal Optimization

SVM Support Vector Machine

WLK Weisfeiler-Lehman Graph Kernel

WL $_{\mathbf{Y V}} \quad$ Deep Weisfeiler-Lehman Kernel by Yanardag and Vishwanathan [85]

kNN $\quad$ Nearest Neighbor

CNN Convolution Neural Network

CW Confidence Weighted classifier

DDG Data Dependency Graph

GUI Graphical User Interface

HI Histogram Intersection

NLP Natural Language Processing

OP Online Perceptron classifier

PA Passive Aggressive classifier

RNN Recurrent Neural Network

RW Random Walk

SP Shortest Path 


\section{List of General Notations}

\begin{tabular}{|c|c|}
\hline$G$ & Program Representation Graph (PRG) \\
\hline$N$ & Set of nodes in PRG \\
\hline$E$ & Set of edges in PRG \\
\hline$\lambda$ & Set of PRG nodes labels \\
\hline$\xi$ & Set of PRG nodes' reachability contexts \\
\hline $\mathscr{G}$ & WLK relabeled PRG \\
\hline $\mathcal{G}$ & CWLK relabeled PRG \\
\hline$f_{c}$ & Label compression function \\
\hline $\mathbf{x}$ & Data instance or sample \\
\hline $\overrightarrow{\mathbf{x}}$ & Feature vector representation of sample $\mathbf{x}$ \\
\hline$y$ & Actual label of a sample \\
\hline$\hat{y}$ & Predicted label of a sample \\
\hline$f(\mathbf{x})$ & Predicted label of sample $\mathbf{x}$ \\
\hline $\mathfrak{f}$ & A feature in $\overrightarrow{\mathbf{x}}$ \\
\hline $\overrightarrow{\mathbf{w}}$ & Weight vector used by linear classifiers \\
\hline$\alpha$ & A support vector instance \\
\hline $\mathbb{X}$ & Set of feature vector representations \\
\hline $\mathbb{V}$ & Vocabulary of features \\
\hline$\langle\cdot, \cdot\rangle$ & Dot product between a pair of samples $h$ \\
\hline$\Phi$ & Feature map used by kernels \\
\hline$k(\cdot, \cdot)$ & Kernel function between a pair of samples \\
\hline
\end{tabular}




$\begin{array}{ll}\mathcal{K}(\cdot, \cdot) & \text { Deep kernel function between a pair of samples } \\ k_{W L}^{(h)}(\cdot, \cdot) & \text { WLK value between a pair of samples at height } h \\ k_{C W L}^{(h)}(\cdot, \cdot) & \text { CWLK value between a pair of samples at height } h \\ \mathbf{k} & \text { Kernel (gram) matrix }\end{array}$





\section{Summary}

Android has evolved to be the most popular mobile operating system over the past several years, since its inception. Millions of Android apps provide a variety of functionalities to users, such as instant messaging, gaming and online shopping. However, due to its prevalence, Android also becomes a prime attack target of cybercriminals. According to Symantec's 2016 Annual Threat Report, 13 million Android malware have been found in the wild, so far. These malware may steal sensitive information, control devices remotely or encrypt on-device data for ransom thus putting the end users at high risk. Besides malware authors, some unscrupulous developers clone the code from popular apps and repackage it with advertisements and new functionalities, thus stealing revenue from the original developers. The sheer volume, growth rate and evolution of malware and clone apps highlight an imperative need for developing effective and scalable automated techniques to detect them.

To perform automated detection, recent approaches both from academia and industry increasingly resort to Machine Learning (ML) techniques. Typically, the detection process involves extracting semantic features from suitable representations of programs (e.g., assembly code, call graphs) and identifying malice or clone code patterns. However, most of these ML algorithms could be applied only on data represented as vectors. Hence, a pivotal factor in determining the effectiveness of these detection processes, is building suitable vector representations of programs. We intend to address this as the primary motive of this thesis. Recognizing that higher level abstractions of programs such as call graphs, controland data-flow graphs retain their semantics well, we intend to learn their representations 
(i.e., graph embeddings) and use them to perform malware and clone detection. We use a common term, Program Representation Graphs (PRGs) to refer to any of the aforementioned graphs. Once appropriate PRG embeddings are built, as a secondary motive, we intend to address specific issues in malware and clone detection processes. In the case of malware detection, three issues: (1) population drift induced by malware's evolution, (2) systematic integration of malware characteristics (i.e., features) from different data sources and (3) precisely locating malicious code portions in PRGs have been addressed. In the case of clone detection, detecting semantic clones (apart from detecting syntactic clones) which remains as a crucial challenge has been addressed. The following are the achievements made in this thesis:

1. We propose a novel graph kernel specifically designed to address the malware detection problem. Previous research has revealed that besides capturing topological neighbourhoods (i.e., structural information) from these graphs it is important to capture the context under which the neighbourhoods are reachable to accurately detect malicious neighbourhoods. We observe that state-of-the-art graph kernels, such as Weisfeiler-Lehman Kernel (WLK) capture the structural information well, but fail to capture contextual in-

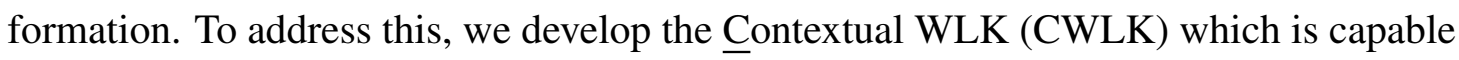
of capturing both these types of information. To the best of our knowledge, this is the first graph kernel specifically addressing a problem from the field of program analysis.

2. It is well-known that malware constantly evolves so as to evade detection. This causes the entire malware population to be non-stationary. Contrary to this fact, most of the prior works on ML based malware detection have assumed that the distribution of the observed malware features does not change or evolve over time. We address the problem of malware population drift and propose a novel online learning based framework to detect malware, named CASANDRA (Context-aware, Adaptive and $\underline{S}$ calable ANDRoid mAlware detector). Towards performing accurate and scalable detection, CASANDRA uses 
CWLK to capture and represent security-sensitive behaviors in PRGs. Towards automatically adapting to population drift, it uses an online classifier. When evaluated with more than 87,000 apps, CASANDRA achieves $89.92 \%$ accuracy, outperforming existing techniques by more than $25 \%$ in their typical batch learning setting and more than $7 \%$ when they are continuously retained.

3. Existing malware detection approaches have typically used classifiers with a variety of features such as security-sensitive APIs, instruction sequences and information flows. These feature sets provide complementary perspectives (interchangeably referred as views) of apps' behaviors with inherent strengths and limitations. Meaning, some views are more amenable to detect certain attacks while they may not characterize several other attacks well. Existing approaches (incl. CASANDRA) use either one or a selected few of the aforementioned feature sets which prevents them from detecting a substantial majority of attacks. To address this, we propose MKLDroID, a unified framework that systematically integrates multiple views in the hopes that, while a malware app can disguise itself in some views, disguising in every view while maintaining malicious intent will prove to be substantially more difficult. MKLDroId leverages on CWLK for extracting PRG embeddings from different views and then employs Multiple Kernel Learning (MKL) to find a weighted combination of the views which yields best detection rates. Besides integrating multiple views, its salient trait is its ability to localize and rank malice code portions (e.g., classes/methods) in PRGs based on their degree of maliciousness.

4. We observe that existing graph kernels such as WLK exhibit an inherent inability to model subgraph-level similarities which results in poor generalization when used with large graphs (such as PRGs). To address this, we propose SubGRAPH2vec, a novel approach for learning latent representations of rooted subgraphs inspired by recent advancements in Deep Learning. We demonstrate that these subgraph vectors could be 
used for building a deep learning variant of WLK. This deep graph kernel exhibits potentials to detect semantic malware and clone variants. Our experiments on several large-scale datasets reveal that SUBGRAPH2VEC achieves significant improvements in generalization and thereby, on accuracies over existing kernels on both malware and clone detection tasks.

In sum, this thesis proposes two methods for learning representations of PRGs namely, CWLK and SubgraPh2vec. With the PRG embeddings thus built, we address four specific issues that plague Android malware and clone detection approaches, namely, population drift, integrating multi-view features for comprehensive detection, localization of malicious code portions and semantics based detection, through leveraging on ML techniques such as Online Learning, Kernel Methods, MKL and Deep Learning. 



\section{Chapter 1}

\section{Introduction}

Over the past few years, Android has evolved to be the most popular mobile Operating System (OS) with nearly 1.8 billion active devices constituting more than $80 \%$ of market share. To serve its vast user base, Google Play, the official market hosts nearly 2 million application (apps) which have penetrated into different walks of life of its users with activities such as social networking, games and online shopping. However, this prevalence has also opened doors for a large number of malicious apps that are capable of inflicting severe damages such as stealing users' private data and controlling devices remotely. For instance, Kaspersky reports [1] discovering nearly 4.1 million new malware in 2016 which is a $17 \%$ increase over 2015. A major reason for such tremendous volume is the production of malware variants. Typically, the attackers produce large number of variants of the same malware by resorting to code transformation and obfuscation techniques. Aggravating the situation, Android app packaging model offers painless and straight-forward opportunities for attackers to piggyback (i.e., inject) malice code on popular benign apps which could then be spread through several third-party markets. Besides malware authors, many deceitful developers exploit this easy repackaging to clone popular apps and inject advertisements or new functionalities and thereby steal revenues from legitimate developers. The mammoth volume and growth rate of malware and clone apps highlights an 
imperative need for developing sound and scalable automated techniques to detect them.

Machine Learning based detection. For over a decade, Machine Learning (ML) techniques have been predominantly used both in academia and industry to perform malware detection. This is because, ML methods automatically learn the characteristics that distinguish malice behaviors, when trained using a collection of malware and benign samples making them amenable for automated detection. Typically, these approaches extract semantic features (e.g., system calls invoked, control- and data-flows, etc.) from suitable representations of programs and apply standard classification algorithms (e.g., Support Vector Machine (SVM), etc.) to perform binary classification. Similarly, in the case of clone detection, semantic representations of apps are subjected to pair-wise comparisons or clustering (using algorithms such as k-means) to detect groups of cloned apps.

Malware and clone detection based on graph representations. Understandably, malware variants perform same or similar malicious functionality, with apparently different syntax, so as to evade syntax-based detectors. However, an important revelation from recent research is that higher level semantic representations such as call graphs (CGs), control- and data-flow graphs mostly stay similar even when the code is considerably altered [26, 29, 32, 63]. In this work, we use a common term, Program Representation Graph (PRG) to refer to any of the aforementioned graphs. As PRGs are resilient against variants, they are being increasingly used to perform malware detection [28, 29, 29]. In essence, such works cast malware detection as a graph classification problem. On the other hand, clone detection techniques also leverage on this invariance property of PRGs and cast semantic clone detection as a graph clustering problem [109].

PRG representations and problem-specific challenges. It is well-known that most of these classification and clustering algorithms could be applied only on data represented as vectors. Hence, a pivotal factor in determining the effectiveness of these detection processes, is building suitable vector representations (aka embeddings) of PRGs. We intend 
to address this as the primary motive of this thesis. Once appropriate PRG embeddings are built, as a secondary motive, we intend to address malware and clone detection problemspecific challenges, separately.

\subsection{Motivations and Goals}

In particular, according to the investigation of ML and PRG based Android malware and clone detection state of affairs, we identify the following problems to be answered in this thesis:

- P1. Building accurate and scalable PRG embeddings. PRGs are rich, complex and highly expressive data structures, facilitating accurate depictions of security-sensitive operations that happen in apps. Building accurate and scalable PRG embeddings that maximally retain their expressiveness is the first and fundamental step to effective malware detection and conducting further research works.

- P2. Adaptive and explainable malware detection. A well-known phenomenon with malware is that their behaviors tend to evolve over time becoming increasingly sophisticated. This renders static detection models obsolete, resulting in gradual degradation of their performance. Hence, to achieve effective detection, a technique must be capable of adapting itself to malware evolution. Furthermore, if it offers humanly comprehensible explanations of its predictions, it will help analysts in studying malware evolution.

- P3. Multi-view malware detection and malicious code localization. Existing malware detection approaches use a variety of features (e.g., system call, API or instruction sequences) characterize and distinguish benign and malice behaviors. These feature sets provide complementary views (or perspectives) of apps' behaviors with inherent strengths and limitations. Understandably, a highly comprehensive and robust detection could be achieved through integrating multiple such views. Also, rather than predicting 
a sample as a whole to be malicious, precisely locating malice code in the PRGs (e.g., classes/methods containing such code) could help analysts to better understand such behaviors and engineer effective counter-measures.

- P4. Learning substructure similarities for better generalization. Many existing embedding approaches decompose graphs into atomic substructures or motifs (e.g., walk, subgraphs, etc.) and represent them as vectors of such motifs. However, the number of unique motifs tend to be extremely large in the case of large datasets, yielding very high dimensional embeddings (see [85]). This hampers the generalization ability and thereby the accuracy of these approaches. This dimensionality issues has to be appropriately addressed for effective detection.

These problems motivate the main works in the thesis. It is non-trivial to solve the aforementioned problems since there exist many technical challenges. We list the following challenges for each problem, respectively:

- C1.1. Expressiveness. PRGs are complex data structures with several attributes that characterize topological relationships among program entities. Representing them as vectors or other formats amenable for applying ML algorithms is a non-trivial task.

- C1.2. Efficiency. The scale of malware detection problem is such that we have millions of samples already and thousands streaming in every day. Many classic graph mining based detection approaches (e.g., [63]) are NP-hard and have severe scalability issues, making them impractical for real-world malware detection [32, 64]. Hence, PRG embedding and subsequent classification or clustering methods have to be highly efficient.

- C2.1. Population drift. Malware apps keep constantly evolving due to various reasons such as exploiting new-found vulnerabilities and evading novel detection techniques. This evolution has a profound impact on malware characteristics and thereby on the features used by ML detection models. This makes the malware features identified today 
unrepresentative of the ones generated in the future. Hence, for effective detection in the wild, it is indispensable for a model to automatically adapt to this population drift.

- C2.2. Explainablity. In general, ML based detection models just predict the labels of a given sample without offering insights or explanations into how those predictions are arrived at. In other words, they act as black-box solutions. However, for malware detection models, understanding the reasons behind their predictions is important in assessing their trustworthiness. This is fundamental if one plans to take action such as deploying a new model or studying malware evolution based on these predictions.

- C3.1. Integrating multiple views. Each of the feature sets mentioned above (API sequences, information flows, etc.) provide a distinctive view of the app. Integrating multiple such views in a systematic and scalable manner in such a way that they complement each other, yielding a comprehensive detection is a highly challenging task.

- C3.2. Malicious code localization. Unlike traditional desktop malware, a substantial portion of code in mobile malware is benign and only a small portion (often called piggybacked portion) is attack related [34, 70-72]. Contrary to this fact, almost all the ML based approaches just predict whether or not a given app, as a whole, is malicious. Such approaches are incapable of locating malice code portions (e.g., classes/methods containing such code). However, precisely locating and ranking malice code portions based on their severity which would cater several important applications is still a largely unexplored and challenging task.

- C4.1. Subgraph similarity. When using PRGs, the efficacy of subgraph features is pivotal in determining the accuracy of the application such as malware or clone detection. Understandably, PRG subgraphs for syntactically different but semantically equivalent code exhibit high similarity. This subgraph level semantic similarities have to be meticulously captured by the PRG embedding approaches to facilitate accurate detection.

- C4.2. Diagonal dominance. Graph kernels (e.g., WLK) which are widely used for graph 
embedding tend to produce extremely high number of motif features when used with large graph datasets. Consequently, only a few motifs will be common across graphs. This leads to a problem such that a given graph is similar to itself but not to any other graph in the dataset. This phenomenon is known diagonal dominance and has to be appropriately addressed to achieve better generalization.

\subsection{Main Works and Contributions}

In the following, four main works are conducted to solve the aforementioned problems and

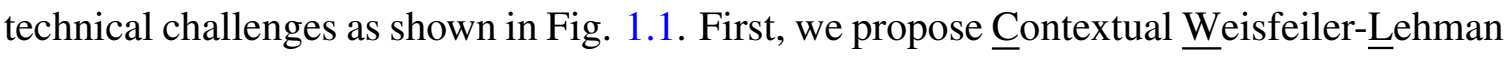
Kernel (CWLK) that is specifically designed to cater malware detection by precisely capturing sensitive information from PRGs. Second, with the proposed kernel, we use an online classifier which is capable of automatically updating its knowledge from samples that stream-in, thus addressing malware population drift. Furthermore, we identify salient PRG subgraph features that led to samples' predictions and thus explain their malice or benign behaviors. Third, we use CWLK to extract five different embeddings of apps with different complementary set of features each representing a semantic view and combine them using Multiple Kernel Learning (MKL). Moreover, we use this multi-view learning to precisely locate malice code portions from PRGs. Finally, to address the diagonal dominance problem in existing graph kernels, we propose an unsupervised representation learning technique to learn subgraph embeddings and build more accurate PRG representations. We elaborate these works as follows:

1. CWLK for malware detection-specific PRG embedding. Building precise and scalable representation of PRGs capturing security-sensitive information could facilitate effective and efficient malware detection. Such a representation could be appropriately leveraged for achieving adaptive, explainable, multi-view detection and malicious code localization. These four motives construct the majority of this thesis. Hence, as the basis of 


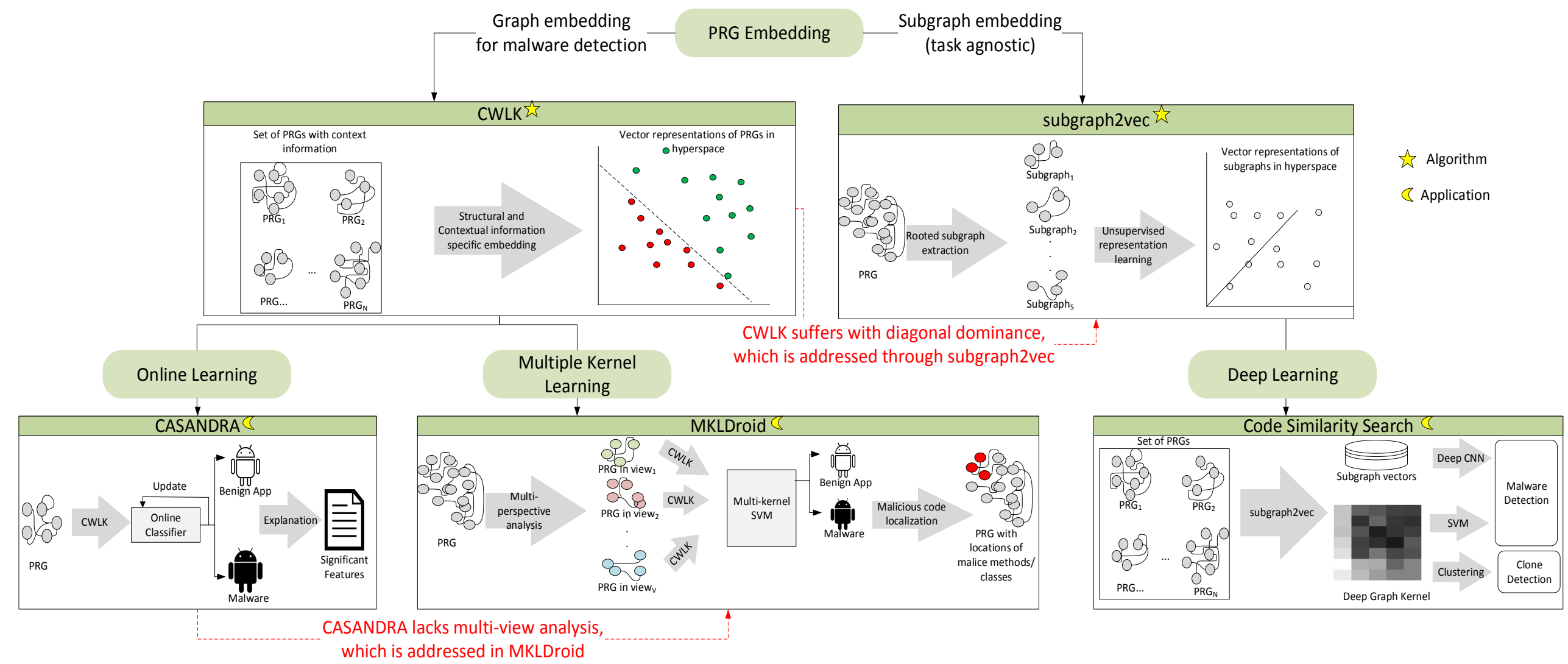

Figure 1.1: Overview of the thesis 
the thesis, we conduct a work to build precise, scalable and task-specific PRG embeddings.

To this end, we note the following revelations from recent research: (i) topological neighbourhoods (i.e., structural information) in PRGs immaculately characterize sensitive behaviors that span across multiple nodes, and (ii) besides capturing sensitive neighbourhoods it is important to capture the context under which they are reachable to accurately determine malicious PRG neighbourhoods. Though existing kernels such as $[74,76-79$, $85,100]$ could capture structural information accurately, they are inherently not equipped to capture contextual information as it is an application- and domain-specific requirement. On the other hand, existing malware detection approaches such $[28,29]$ could capture contextual information, but fail to appropriately model structural information. Bridging this research gap, we develop CWLK, a graph kernel capable of capturing both these types of information.

Contributions. Thus, we make the following contributions in the first work:

- We develop CWLK, a graph kernel specifically designed to perform accurate and scalable malware detection. To the best of our knowledge, this is the first kernel specifically addressing a problem from the field of program analysis.

- Through large-scale experiments and comparative analysis, we show that the proposed kernel outperforms two state-of-the-art graph kernels and three malware detection solutions in terms of accuracy, while maintaining high efficiency.

- We make an efficient implementation of the proposed kernel publicly available at: https://sites.google.com/site/cwlkernel

2. Adaptive, Scalable and Explainable Malware Detection. Leveraging on the proposed CWLK, we intend to address the problem of malware population drift. To this end, we combine CWLK with online learning, a ML paradigm which is inherently capable of handling such drift and develop an adaptive, scalable and explainable malware detection 
framework named CASANDRA. The framework deploys CWLK to extract contextual API dependencies from PRGs facilitating accurate and scalable detection. Besides this, CASANDRA has specific advantages: (i) being adaptive to the evolution in wild malware samples over time (ii) explaining the salient features that led to an app's classification as being malicious or benign. In a large-scale comparative analysis, with more than 87,000 wild apps, CasanDRA achieves $89.92 \%$ accuracy, outperforming existing techniques by more than $25 \%$ in their typical batch learning setting and more than $7 \%$ when they are continuously retained, while maintaining comparable efficiency. In sum, CASANDRA demonstrates potentials as a reliable framework to perform market-scale analysis.

Contributions. We make the following contributions in the second work:

- We propose and develop CASANDra, a context-aware, adaptive, scalable and explainable Android malware detection framework which is based on online learning, where we do not assume that the malware population is stationary.

- Performing explainable malware detection is a salient feature of CASANDRA. As we demonstrate through our experiments, CASANDRA's explanations are more comprehensive and semantically closer to malicious behaviors than those of state-of-the-art approaches.

- Adaptiveness is an unique feature of Casandra. We thoroughly demonstrate how CASANDRA adapts to malware population drift through carefully designed experiments with real-world malware. To the best of our knowledge, none of the existing approaches possess this capability.

3. Multi-view Malware Detection and Malicious Code Localization. Even though CASANDRA could adapt itself to malware population drift, it captures only one set of features (i.e., API dependencies) and therefore one semantic view of the app. This results in sub-par detection rates. However, thanks to their expressiveness, PRGs facilitate capturing multiple set of features to provide more comprehensive detection. To this end, in our 
subsequent work, we propose MKLDroid framework, which uses CWLK to extract five different features sets (each representing a distinct view), namely, API dependencies, permission dependencies, information flows, instruction sequences and control flow structures and subsequently, applies MKL to integrate them in a scalable and systematic manner. The intuition behind MKLDroID is that, while a malware app can disguise itself in some views, disguising in every view while maintaining malicious intent will prove to be substantially more difficult. Besides comprehensive detection, MKLDROID's unique and salient trait is its ability to locate fine-grained malice code portions leveraging on its multi-view analysis. It awards a maliciousness score to every method and class in a predicted malware app and thereby ranks them according to their degree of maliciousness. Such malicious code localization helps immensely in understanding behaviors of malware variants and building malware signatures.

Contributions. We make the following contributions in the third work:

- We propose MKLDRoId, a malware detection framework which integrates multiple semantic views using MKL thereby extracting a richer representation. This helps MKLDRoID achieve superior accuracies over approaches which use either one or a selected few of the views.

- We propose a novel kernel methods based approach to locate and rank malicious code portions (i.e., methods/classes) in PRGs. To the best of our knowledge, ours is the first approach that performs automatic multi-view malicious code localization.

- We contribute to future research on malicious code localization by releasing MKLDroID's results. In particular, all the identified malice classes and methods from apps in two well-known benchmark datasets are made available at: https://sites . google.com/view/mkldroid/home.

\section{Learning Distributed Representations of Rooted Subgraphs.}


Though existing kernel such as WLK and our proposed kernel, CWLK could accurately represent PRGs, when used with large datasets, they suffer with two of the problems mentioned in $\S 1.1$, namely, inability to capture substructure similarity (challenge 4.1) and diagonal dominance (challenge 4.2). We address these challenges in our final work. To this end, we propose, SUbGraph2vec, a novel approach for learning latent representations of rooted subgraphs from large graphs inspired by recent advancements in Deep Learning. Subgraph2vec leverages on local information obtained from neighbourhoods of nodes and subgraphs to learn their distributed representations in an unsupervised fashion. We demonstrate that subgraph vectors learnt by our approach could be used for building a deep learning variant of WLK. Our experiments reveal that SUBGRAPH2VEC achieves better generalization and thereby yielding significant improvements in accuracies over existing graph kernels (incl. WLK) on both malware and clone detection tasks.

Contributions. Thus, we make the following contributions in the fourth and final work:

- We propose Subgraph2vec, an unsupervised representation learning technique to learn embeddings of rooted subgraphs present in large graphs and also build a deep learning variant of WLK.

- Through our large-scale experiments on several benchmark and real-world datasets, we demonstrate that SubgraPh2vec could significantly outperform state-of-the-art graph kernels on malware detection (graph classification) and clone detection (clustering) tasks.

- We make an efficient implementation of Subgraph2vec code (along with relevant libraries) available at: https://sites.google.com/site/subgraph2vec. 


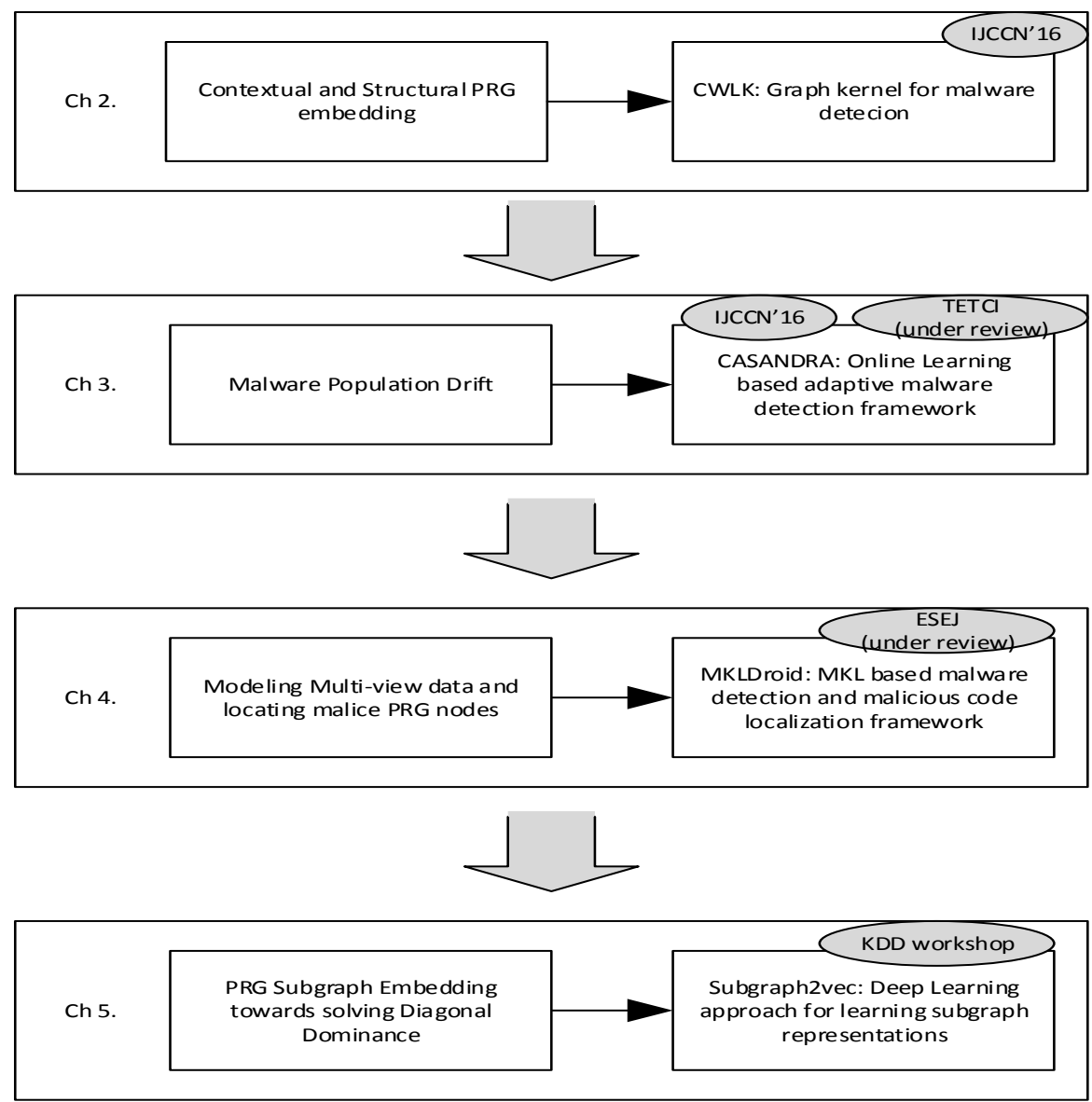

Figure 1.2: Road-map of contribution chapters in this dissertation

\subsection{Thesis Outline}

Fig. 1.2 shows the roadmap for this thesis, which describes the sequence of our research work and thereby reveals the train of our thoughts on PRG based malware and clone detection. The conference and journal publications made as a part of these works are also highlighted. The remaining of the thesis is organized as follows:

In Chapter 2, we discuss the background and related work on two topics: (i) Android security and (ii) relevant ML algorithms and paradigms. In particular, we introduce the status quo of Android malware and clone detection approaches, preliminaries on ML topics such as kernel methods, MKL and representation learning. 
In Chapter 3, we present CWLK, the kernel we propose to embed PRGs specifically catering the malware detection task.

Chapter 4 describes the CASANDRA framework that uses CWLK along with online learning to handle malware population drift and perform context-aware, adaptive, scalable and explainable detection.

In Chapter 5, we present MKLDroid framework that uses CWLK along with MKL to systematically integrate data from multiple semantic views of apps to perform comprehensive malware detection and malicious code localization.

In Chapter 6, we propose SubgraPh2vec, an unsupervised subgraph representation learning approach to build deep graph kernels. We demonstrate Subgraph2vec's ability to achieve better generalization and thereby better malware and clone detection accuracies than the existing kernels.

In Chapter 7, we conclude the works in this thesis, and propose the potential directions of our works in the future. 


\section{Chapter 2}

\section{Background and Preliminaries}

In this chapter, the background on Android system, attacks and defense (i.e., malware/clone detection methods) and preliminaries on Machine Learning paradigms used in this thesis are discussed.

\subsection{Background on Android Security and Defense}

\subsubsection{Android System}

Android is a Linux-based mobile OS developed by the Open Handset Alliance (led by Google) since 2008. As of this writing, it is the most popular mobile OS with more than $86 \%$ of market share [3] running not only on mobile phones, but also on the other platforms such as smart TVs, watches and tablets. Android's architecture is shown in Fig. 2.1. Basically, Android consists of four layers. The bottom layer is Linux kernel, where drivers and system-level services are running. The upper level deploys system libraries and Android Dalvik Virtual Machine (DVM), which provides a variety of functionalities and runtime environment for Android applications. There are many services written in Java in the application framework layer. For example, activity manager is used to manage activi- 


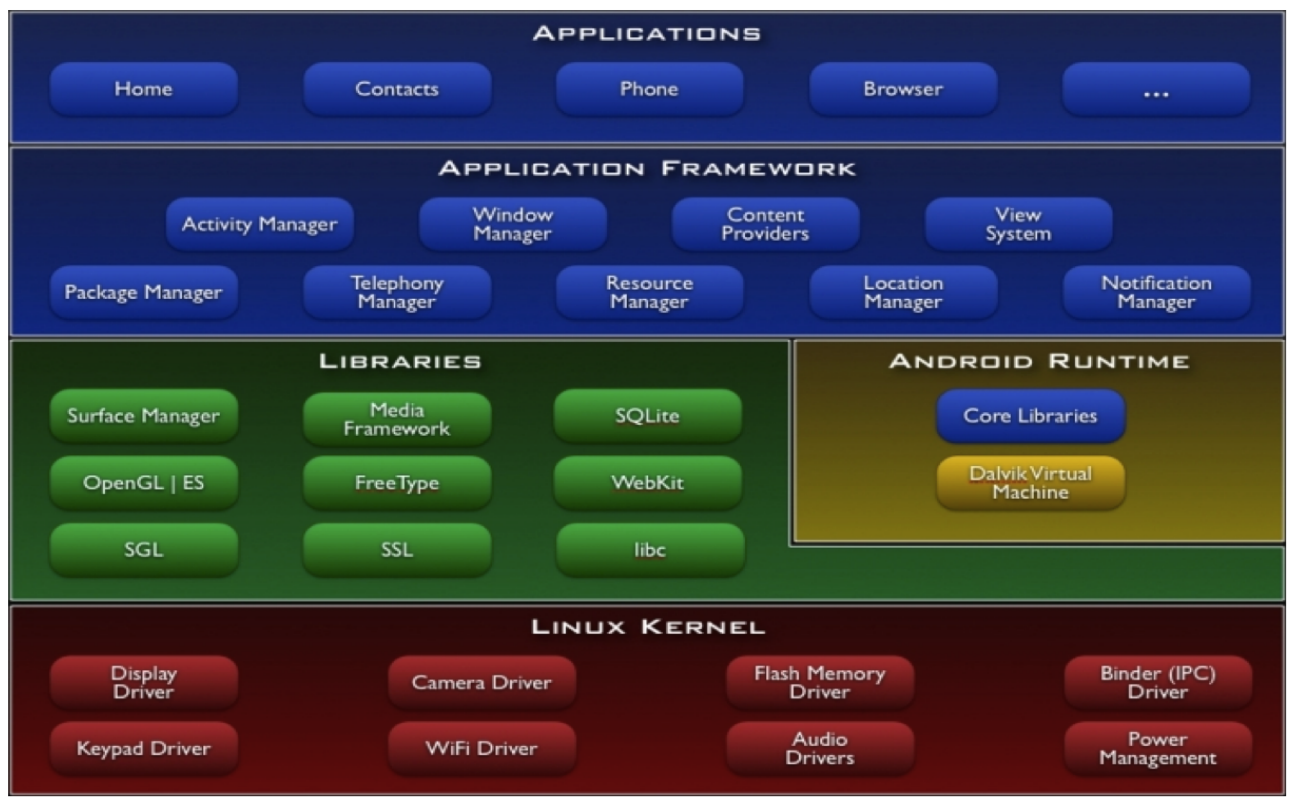

Figure 2.1: Android OS Architecture

ties in the system. The top layer is application layer, where Android apps are supposed to run. Apps can invoke APIs or services in the framework layer and library layer to complete their functionalities.

Android Apps. Apps designed to run on Android devices are predominantly written in Java, and comprise of four building blocks: activity, service, broadcast receiver, and content provider. An activity is the app's entry point for interacting with the user. It usually has a graphical interface; a service is a component that runs in the background supporting long-running or expensive operations (e.g., downloading a file); a broadcast receiver is used to listen to the broadcast messages sent either by the system or other apps (e.g., receiving an email or SMS), and facilitates the apps to react with predefined actions, and; a content provider is used to manage a shared set of app data that once can store in the file system.

\subsubsection{Android Malware Detection}

We have witnessed the rapid development and evolution of Android malware since the first Trojan malware was discovered in 2010 [23]. Android is facing a variety of threats that can 
disturb users' normal life. In this section, we discuss the background on four popular types of Android malware.

Privacy leaks. Privacy leaking malware have received much public attention. They may steal sensitive information from Android devices, such as SMS messages, contact information, geographic locations and call $\operatorname{logs}[24,25]$. The stolen information can be used to track users, make profits, obtain Mobile Transaction Authentication Number (mTAN) and so on. Privacy leakage constitutes a large portion of Android malware (about nearly $80 \%$ of malware in the dataset released by the seminal work, GENOME [24], involve in privacy leaks).

Privilege escalation. It is common to grant privileges (i.e., permissions) to apps during installation or execution to access sensitive services and data. However, vulnerabilities in apps could result in unwanted escalations of privilege authorizations, allowing the apps to perform actions that are not intended by the user. In principle, permissions must be explicitly identified and apps cannot access the resources until the installer grants it the required permissions. However, many malicious apps circumvent the permission mechanism and exploit indirect tactics to access sensitive resources. For instance, as reported in Chabada [43] an app, disguising itself a restaurant recommender, could escalate its privileges easily to read user's phone number and accounts information.

Financial charge. Premium Rate Services are value-added services offered by a telecom provider. Examples of PRS include subscriptions to information, services of gaming, charity donations and so forth, which charge users beyond the standard network charges. Android malware can automatically and stealthily text or call a premium number, and cause additional fees $[24,28]$.

Phishing. This attack uses social engineering techniques and disguises malware to be a normal app, which tricks users into exposing their credentials. Phishing attack is becoming progressively severe, especially after it targets the financial apps [20]. SmiShing, a kind 
of phishing attacks, spreads fake SMS messages to users and tricks them into opening the crafted phishing web page and entering their credentials. In addition, malware can also mimic target apps (e.g., banking apps and social apps) installed on device. The credentials entered by users in the fake app will be sent to the attacker.

In summary, one could observe that malware variants involved in the aforementioned attacks often share similar code, especially while carrying out the same family of attack. Almost all the existing ML and signature based detection techniques (reviewed in §2.1.4) use a code similarity evaluation method (or sometimes develop their own method) to identify portions of code that are similar to known/reported attack codes. To this end, we propose an effective method to identify similar malice code from PRGs in chapter 3 and use the same to identify malware in chapters 4 and 5, addressing practical malware attacks related issues along the way. Finally, in chapter 6, we extend the method proposed in chapter 3 to detect not just malware but also code clones.

\subsubsection{Android Clone Detection}

Clones are nothing but similar snippets of source code, byte code or other intermediate representation of code. Given the ease of reverse engineering Android apps, attackers clone the code from popular legitimate apps and repackage it with monetization code (e.g., ad libraries), purpose-added functionalities or even malicious code. The resulting repackaged apps are known as clone apps or piggybacked apps. Subsequent to cloning an app, attackers would upload it to the same to third-party or official markets. App clones bring the following problems:

(i) Legitimate developers lose their ad revenue and user bases to attackers. According to [24], $14 \%$ of the ad revenue and $10 \%$ of the user base for a legitimate developer are diverted to clone app developers.

(ii) Exploiting on the popularity of benign apps, malware authors piggyback malice code 
on to them and use them for propagation. For instance, in the GENOME dataset [24], around $86 \%$ of the malware apps are nothing but repackaged variants of benign apps.

(iii) Considering the volume of apps in the official and third-party markets is in the order of millions, the clone detection methods need to be extremely scalable.

These threats highlight the motivations to detect and contain them, in a timely manner.

Type4 of clone apps. In general, while detecting app clones from byte code or other similar presentations, we encounter the 4 following types of clones:

Type 1 clones: Identical byte code fragments except for variations in layout.

Type 2 clones: Syntactically identical code fragments except for variations in name of variables, identifiers, literals, types in addition to Type 1's variations.

Type 3 clones: Copied code fragments with further modifications such as changed, added or removed statements in addition to Type 2's variations.

Type 4 clones: Code fragments that perform similar functionalities but implemented through different syntactic variants.

Thus, it is clear that detecting type 1,2 and 3 clone are relatively easy compared to type 4 clones.

Other challenges. A majority of the existing approaches such as [110] and [109] confine to detecting the first three types. In this thesis, we take the first steps in developing Deep Learning based methods to detect type 1, 2 and 3 clones. We intend to explore extending the same approach towards detecting type 4 clones in the future. Also, given the scale of the cross-market clone detection problem, performing a pair-wise comparisons of apps to detect clones (as discussed in [111]) is an impractical approach.

In summary, it could be seen that for detecting app clones, one needs an effective representation of code (or code fragments), leveraging which one could evaluate the similarities among a large collection of apps. To this end, we develop representation techniques in chapter 6 and use clustering to identify clone apps from a given collection, avoiding the 
need for a pair-wise comparison.

\subsubsection{Defense: Existing Techniques and Analysis Paradigms}

There are a plenty of defense techniques against Android malware and clones. In general, they leverage one of the two following analysis paradigms to extract semantic features required for their detection: (i) static analysis, and (ii) dynamic analysis.

Static Analysis. Static analysis based clone and malware detection techniques inspect Android apps without executing them. Generally, they achieve better code coverage and scalability compared to dynamic analysis, as they acquire access to almost all of apps' byte code through disassembly and reverse engineering. However, they are susceptible to obfuscation techniques such as dynamic code loading and reflection, making them imprecise. Majority of existing malware detection (e.g., [25, 28, 29, 31, 32] ) and almost all the existing clone detection techniques (e.g., [109-111]) leverage this paradigm. These methods extract features such as API sequences, permissions, instructions, control- and data-flows and use them for detecting malware and clones.

Dynamic Analysis. Different from static analysis, dynamic analysis needs to execute apps (in a controlled environment) to get evidence with regard to the analysis target. Typically, in this paradigm, Android apps are installed in an emulator or a real mobile device, and executed with a certain crafted input. Dynamic analysis either collects the execution traces or tracks the flow of sensitive information, and then determine whether or not the analyzed app is malicious. Dynamic analysis overcomes the shortcomings of static analysis such as tracking dynamically loaded code, but is limited by poor scalability and code coverage. TaintDroid [62], CrowdDroid [60], and BayesDroid [61] are typical examples of dynamic analysis based malware detection methods. They extract features such as execution traces and system call sequences for precise detection. Due to their poor code coverage, dynamic analysis is not used for app clone detection. 
In summary, due to their excellent scalability and reasonably good code coverage, we prefer using static analysis over dynamic analysis for our clone and malware detection works in this thesis.

\subsection{Machine Learning Preliminaries}

In this subsection, preliminaries on ML paradigms such as kernel methods, MKL, online learning and representation (deep) learning which lay the fundamentals of algorithms proposed and application-specific issues addressed in this thesis are provided.

\subsubsection{Kernel Methods and Graph Kernels}

Kernel methods have been highly successful in solving a specific class of problems where feature vector representations of samples are not readily obtainable. Malware detection using PRGs is one of such problems. For many well-known classifiers, the data samples have to be explicitly represented as feature vectors through a user-specified feature map, $\Phi$. In contrast, kernel methods require only a user-specified kernel $k$, i.e., a similarity function over pairs of samples in their native representations. Kernel methods work by mapping the samples into a feature space, implicitly and finding an appropriate decision boundary in the new feature space. Here, feature map $\Phi(\cdot)$ is realized through the kernel function $k$, which facilitates computing inner products in the feature space using the samples in their native representation, i.e., for a pair of samples $x_{i}$ and $x_{j}, k\left(x_{i}, x_{j}\right)=\left\langle\Phi\left(x_{i}\right), \Phi\left(x_{j}\right)\right\rangle$. The kernel function must be positive definite for most kernel classifiers. Examples of positive definite kernels are the Dirac, Histogram Intersection (HI) and Gaussian kernels [133]. Roughly speaking, a kernel value is a measure of similarity between a pair of samples. Therefore, a graph kernel is a similarity measure between graphs.

Graph Kernels. Formally, a graph kernel $k: \mathbb{G} \times \mathbb{G} \rightarrow R$ is a kernel function defined 
on a domain of graphs, $\mathbb{G}$. Hence, given a labeled graph dataset $D_{g}=\left(g_{1}, y_{1}\right), \ldots,\left(g_{n}, y_{n}\right)$ and a graph kernel $k$, a kernel classifier (e.g., SVM) can be directly used to perform graph classification. Graph kernels usually belong to the family of $R$ convolution kernels. These kernels decompose graphs into substructures such as walks, subgraphs etc. The comparison of two graphs is then based on the similarity between all pairs of such substructures. Several graph kernels have been proposed based on this idea [74, 76-79].

Explicit vs. Implicit Feature mapping. Existing graph kernels can be classified into approaches that use explicit feature mapping $(\Phi)$ and those that directly compute a kernel function (i.e., $\Phi$ is not necessarily known and may be of infinite dimension) [73]. Examples of former category include Weisfeiler-Lehman Kernel (WLK) [77], Neighborhood Hashing Graph Kernel (NHGK) [78] and CWLK, and that of latter category are Random Walk (RW) [74] and Shortest Path (SP) [76] kernels. Kernels that support explicit feature mapping have two advantages that make them particularly suitable for the malware detection problem:

(1) Scalability. If explicit representations are manageable, these approaches usually outperform other kernels regarding runtime on large datasets, since the number of vector representations scales linear with the dataset size $[73,77]$.

(2) Explainability. These kernels support extracting substructures of graphs as features and building a vocabulary of such features. This facilitates building explicit feature vector representation of individual graphs $[77,85]$. This aspect makes this category of kernels amenable for performing explainable malware detection [73].

\subsubsection{Multiple Kernel Learning}

Given a set of $m$ kernels, MKL is an ML paradigm which aims to arrive at a combination of these kernels that maximizes accuracy at a reasonable efficiency. Each of the $m$ kernels are referred as base kernels in the MKL setting. The following are the main use cases of 
MKL: (1) combining data from different sources (e.g., static and dynamic analysis of apps) and (2) given a large set of base kernels, arriving at optimal combination of these kernel and parameters in scalable manner. When combining data from different sources, each source may represent a perspective or view of the data with different modalities. Hence, MKL could be considered as a principled way of performing multi-view learning with multi-modal data.

Many MKL algorithms have been proposed to facilitate classification and clustering tasks through both linear and non-linear combinations of base kernels. In this work, we have focused on using linear MKL classification algorithms. This is because, they facilitate explainable learning (see $§ 5.3 .4$ and $§ 5.3 .5$ for details) compared to their non-linear counter parts.

The basic idea behind linear MKL algorithms is to add an extra parameter that signifies the weights of base kernels to the minimization problem of the learning algorithm. The combined kernel is computed as follows:

$$
\mathbf{k}_{\text {comb }}=\sum_{v=1}^{m} \beta_{v} \mathbf{k}_{v}
$$

where $\beta_{v}$ is the weight of base kernel $\mathbf{k}_{v}$.

Standard optimization methods could be leveraged to solve this optimization problem. Adaptations of existing techniques such as Sequential Minimal Optimization (SMO) have also been developed for multiple kernel SVM-based methods [92]. Once the combined kernel $\mathbf{k}_{\text {comb }}$ is computed, it could be used with a kernel classifier to perform multi-view multi-modal classification. For details on the optimization techniques to solve eq. (2.1), alternative choices of MKL algorithms and discussions on MKL applications, we refer the reader to an excellent survey by Mehmet et al. [91]. 


\subsubsection{Online Learning}

Majority of the classification algorithms such as SVMs, RFs and Logistic Regression are batch learners and are typically unsuitable for classifying a stream of samples. However, in the applications that we consider, namely, malware and clone detection, it is not possible to have a collection of all the apps beforehand and then proceed with detection. Samples keep streaming-in over time. Hence, we advocate replacing batch learning with another incremental learning paradigm, namely, online learning, for effective real-world malware and clone detection. The fundamental differences between these two paradigms are as follows:

Batch Vs. Online Learning. Batch-learning algorithm uses a batch of labeled samples to initially train its model, which subsequently is used to predict the labels of new samples. After some time, the model is re-trained using a new batch of labeled samples. On the other hand, an online algorithm continuously retrains its model upon receiving each labeled sample and makes prediction of a new samples using the latest updated model. Batch-learning algorithms require significantly more memory and training time than online algorithms as they require a batch of data for training a model. Batch learners typically take multiple passes over the data, as it is available in totality and optimize their parameters to achieve high accuracies. On the contrary, online learners take only one pass over the samples (i.e., each samples is used exactly once for training the model).

Given a set of samples $\mathbb{X}=\left\{\mathbf{x}_{1}, \mathbf{x}_{2}, \mathbf{x}_{3}, \ldots\right\}$ and corresponding labels, $\mathbb{Y}=\left\{y_{1}, y_{2}, y_{3}, \ldots\right\}$, where $y_{i} \in\{+1,-1\}$, the goal of batch learners is to learn a model $f: \mathbb{X} \rightarrow\{+1,-1\}$ such that, the classification loss (i.e., error) among all the samples of $\mathbb{X}$ is minimum (often regularization is used to mitigate overfitting and hence the classification loss need not be exactly minimal, always. It could be fine-tuned with techniques such as cross-validation [25]).

On the other hand, online learners receive a stream of sample-label pairs, 
$\left\{\left(\mathbf{x}_{1}, y_{1}\right),\left(\mathbf{x}_{2}, y_{2}\right), \ldots\right\}$, where $\mathbf{x}_{t}$ denotes a sample received at time $t$ and its label is of the form $y_{t} \in\{+1,-1\}$. We denote the feature vector representation of sample $\mathbf{x}_{t}$ as $\overrightarrow{\mathbf{x}}_{t}$. At each time step $t$ during training, the algorithm makes a label prediction $f_{t}\left(\overrightarrow{\mathbf{x}}_{t}\right)$, which for linear classifiers is $f_{t}\left(\overrightarrow{\mathbf{x}}_{t}\right)=\operatorname{sigh}\left(\overrightarrow{\mathbf{w}}_{t} \cdot \overrightarrow{\mathbf{x}}_{t}\right)$. $\overrightarrow{\mathbf{w}}$ is a weight vector that signifies the weight (i.e., relative importance) of each feature. After making a prediction, the algorithm receives the actual label $y_{t}$. (If $f_{t}\left(\overrightarrow{\mathbf{x}}_{t}\right) \neq y_{t}$, an error is recorded for time $t$.) Then, the algorithm constructs the hypothesis for the next time step $f_{t+1}$ using $f_{t}, \mathbf{x}_{t}$ and $y_{t}$. This lightweight training and update procedures of online learners make them amenable for classifying and learning from a high volume, high speed stream of samples. Such a stream is what we encounter in our malware and clone detection applications.

Now, we introduce and briefly review three well-known online learners that we experimented with, in the forthcoming chapters.

1. Online Perceptron (OP) [114]. OP is a classic linear classifier that makes the following update to the weight vector whenever it makes a mistake:

$$
\overrightarrow{\mathbf{w}}_{t+1} \leftarrow \overrightarrow{\mathbf{w}}_{t}+y_{t} \overrightarrow{\mathbf{x}}_{t}
$$

The model (i.e., weight vector) is of the form $\overrightarrow{\mathbf{w}}=\left[w^{(1)}, w^{(2)}, w^{(3)}, \ldots\right]^{T}$, where $w^{(\mathfrak{f})}$ denotes the significance of feature $\mathfrak{f}$. The advantage of the Perceptron is its simple update rule. However, because the update rate is fixed, the Perceptron cannot account for the severity of the misclassification. As a result, the algorithm can overcompensate for mistakes in some cases and under-compensate for mistakes in others.

2. Passive-Aggressive (PA) algorithm [116]. PA addresses the above said shortcoming of OP. The objective of this algorithm is to change the model as minimal as possible to compensate for any mistake and low-confidence prediction it makes. Specifically, with 
each given sample PA solves the following optimization:

$$
\overrightarrow{\mathbf{w}}_{t+1} \leftarrow \overrightarrow{\mathbf{w}}_{t}+\alpha_{t} y_{t} \overrightarrow{\mathbf{x}}_{t}
$$

where $\alpha_{t}$ is defined as $\max \left\{\frac{1-y_{t}\left(\overrightarrow{\mathbf{w}}_{t} \cdot \overrightarrow{\mathbf{x}}_{t}\right)}{\left\|\overrightarrow{\mathbf{x}}_{t}\right\|^{2}}, 0\right\}$

The updates in eq. (2.3) occur only when the dot product does not exceed a fixed confidence margin - i.e., $y_{t}\left(\overrightarrow{\mathbf{w}}_{t} \cdot \overrightarrow{\mathbf{x}}_{t}\right)<1$. The PA algorithm has been successful in practice because the updates explicitly incorporate the notion of classification confidence. However, PA attempts to update the model only when it commits a misclassification or a low confidence prediction. Meaning, the model does not reward itself when it makes correct classifications with high confidence, but just penalizes itself aggressively for misclassifications.

3. Confidence Weighted (CW) learner [117]. The CW learner addresses the abovesaid limitation of PA. The idea behind CW leaner is to maintain a different confidence measure for each feature so that less confident weights are updated more aggressively than more confident weights. The standard deviation update rule for $\mathrm{CW}$ is similar in spirit to PA. However, instead of describing each feature with a single coefficient, CW describes per-feature confidence by modeling uncertainty in weight $w^{(i)}$ as follows.

A Gaussian distribution over the weights with mean $\vec{\mu}$ and covariance matrix $\Sigma$ is maintained by the $\mathrm{CW}$ algorithm. The value $\mu^{(\mathfrak{f})}$ represents mean weight of feature $\mathfrak{f}$ (i.e., mean of $\left.w^{(\mathfrak{f})}\right)$, and the value $\Sigma_{\mathfrak{f}, \mathfrak{f}}$ captures the confidence in $\mathfrak{f}$ 's weight. While classifying a new sample $\mathbf{x}$, the weight $\overrightarrow{\mathbf{w}}$ is drawn from $N(\vec{\mu}, \Sigma)$. In practice, one could choose $\overrightarrow{\mathbf{w}}=\vec{\mu}$, the average weight vector and use the function $\operatorname{sign}\left(\overrightarrow{\mathbf{w}}_{t} \cdot \overrightarrow{\mathbf{x}}_{t}\right)$ to arrive at the predicted label. CW updates the model, (i.e., $\vec{\mu}$ and $\Sigma$ ), continuously on every labeled sample instead of only when committing misclassifications. The rationale is that making correct prediction also suggests that the model should increase its confidence of the current weights. CW's update 
rule is as follows:

$$
\begin{gathered}
\left(\vec{\mu}_{t+1}, \Sigma_{t+1}\right)=\arg \min _{\vec{\mu}, \Sigma} D_{K L}\left(\mathcal{N}(\vec{\mu}, \Sigma) \| \mathcal{N}\left(\vec{\mu}_{t}, \Sigma_{t}\right)\right), \\
\text { s.t.P } \operatorname{Pr}_{\overrightarrow{\mathbf{w}} \sim N(\vec{\mu}, \Sigma)}\left[y_{t}\left(\overrightarrow{\mathbf{w}} \cdot \overrightarrow{\mathbf{x}}_{t}\right)\right] \geq \eta .
\end{gathered}
$$

Eq. (2.4) determines that the new distribution characterized by new $\Sigma$ and $\vec{\mu}$ should be close to the old distribution as much as possible. The Kullback-Leibler (KL) divergence $\left(D_{K L}\right)$ provides the measure of distance between the two distributions. Eq. (2.5) determines that the update should ensure that the probability of making correct prediction if the same sample, $\mathbf{x}_{t}$ is seen in the next round must be bigger than $\eta$, where $\eta$ is a configurable parameter (usually set bigger than 50\%). The computational complexity of the update is linear in the number of non-zero features in $\mathbf{x}_{t}$.

The strength of CW lies in its notion of modeling confidence on features' weights. Evidently, if the weight of a feature does not fluctuate very much over time, one could confidently believe that this weight is what it should be. CW achieves the two following desirable characteristics through this confidence notion, which are not exhibited by both OP and PA:

- The weights of more confident features are updated less aggressively. This makes CW naturally robust to labeling noise.

- The model is updated just enough to adapt to the changing trends in the data while refraining from changing too much. The rationale is that the previous model carries valuable information about the data and should not be modified too abruptly. 


\subsubsection{Representation (Deep) Learning}

Typically, machine learning and PRG based clone and malware detection techniques use hand-crafted features such as PRG node labels, paths and subgraph patterns. These features help to capture structurally similar features. However, they are not capable of effectively capturing semantic similarity among PRG substructures. For instance, two graph portions containing getLastKnownLocation and getLatitude APIs should be considered as exhibiting high similarity as these API render semantically similar functionality. However, PRG node kernels such as WLK [77], NHGK [78] and CWLK and other approaches that consider node labels as features such as AppContex [28] tend to consider them as exhibiting no similarity at all (i.e., kernel values will be zero).

This limitation could be addressed by replacing these hand-crafted features with the ones that are learnt automatically from data itself. This process of learning features is known as representation learning and it typically requires very high volume of data to learn meaningful features [123]. Basically, there are two subcategories of this process, namely, supervised and unsupervised representation learning. Deep Learning techniques such as auto encoders, Convolution Neural Nets (CNNs) and Recurrent Neural Nets (RNNs) are popularly used for representation leaning [123]. In supervised representation learning, large amount of labeled data (e.g., PRGs labeled as malware or benign, images labeled with objects contained in them) are used with learners such as CNNs and RNNs to learn representations of samples that are specific to a task (e.g., malware detection, image categorization). A major bottleneck in such approaches is that, acquiring large amounts of labeled data is an expensive and often laborious and time consuming task. On the other hand, unsupervised representation learning leverages large and easily available unlabeled data to learn features that are task-agnostic. Representation learning has outperformed hand-crafted features by very high margins in fields such as Computer Vision and Natural Language Processing (NLP). For instance, in NLP, word embeddings have been used to 
replace hand-crafted features like word and character n-grams, parts of speech tags etc. in tasks such as sentiment analysis and text summarization where semantic similarity is of more importance than syntactic similarity [124]. This inference is directly relevant to many program analysis tasks such as malware and clone detection.

Recently, several techniques have been proposed to learn representation of substructures from graphs such as nodes [103], walks [125] etc. They have been deployed in graph mining tasks like node classification, link prediction and community detection and have produced excellent results. In this thesis, we propose a technique to learn representations of subgraphs that has shown potentials to yield better accuracies than hand-crafted features used by kernels such as WLK on both clone and malware detection tasks. 


\section{Chapter 3}

\section{Contextual Weisfeiler-Lehman Kernel for Malware Detection}

In this chapter, CWLK, a graph kernel that we propose to embed PRGs specifically to address the malware detection problem is presented. The contents of this chapter as presented in a paper titled 'Contextual Weisfeiler-Lehman Kernel for Malware Detection' in IJCNN 2016. Another paper of ours titled 'Context-aware, Adaptive and Scalable Android Malware Detection' which is published in IEEE Trans. of Emerging Topics in Computational Intelligence uses a portion of this chapter.

\subsection{Introduction}

Malware detection has evolved as one of the challenging problems in the field of cybersecurity as the attackers continuously enhance the sophistication of malware to evade novel detection techniques. Malware for various platforms such as desktop, web and mobile devices is growing at an alarming rate. For instance, Kaspersky reports [1] discovering nearly 4.1 million new malware in 2016 which is a $17 \%$ increase over 2015 . This volume and growth rate clearly highlights an imperative need for automated malware detection solutions.

To perform automated malware detection, security analysts from both academia and in- 
dustry, resort to program analysis and ML techniques. Typically, this process involves extracting semantic features from suitable representations of programs (e.g., assembly code, CGs) and detecting malicious code or behavior patterns using ML classifiers [25, 26, 28, $29,32,38]$.

A major reason for such tremendous growth rate in malware is the production of malware variants. Typically, the attackers produce large number of variants of the same malware by resorting to techniques such as variable renaming and junk code insertion $[25,29,32,63]$. These variants perform same malicious functionality, with apparently different syntax, thus evading syntax-based detectors. However, PRGs (introduced in chapter 1) such as CGs or CFGs mostly stay similar even when the code is considerably altered $[26,29,32,63]$.

As PRGs are resilient against variants, many works in the past have used them to perform malware detection. In essence, such works cast malware detection as a graph classification problem and apply existing graph mining and classification techniques [63]. Some methods such as $[26,28,32]$ note that ML classifiers are readily applicable on data represented as vectors and attempt to encode PRGs as feature vectors. Particularly, these techniques face two challenges:

- (C1.1) Expressiveness. PRGs are complex and expressive data structures that characterize topological relationships among program entities. Representing them as vectors is a non-trivial task. In many cases vectorial representations of PRGs fail to capture all the vital information. For instance, AppContext [28], a well-known Android malware detection approach represents apps as PRGs and ends up capturing features from individual nodes without their topological neighbourhood information. With such loss of expressiveness, attacks that span across multiple PRG nodes could not be effectively detected.

- (C1.2) Efficiency. The scale of malware detection problem is such that we have millions 
of samples already and thousands streaming in every day. Many classic graph mining based approaches (e.g., [63]) are NP-hard and have severe scalability issues, making them impractical for real-world malware detection [32, 64].

Graph kernels for malware detection. One of the increasingly popular approaches in ML for graph-structured data is the use of graph kernels. Recently, efficient and expressive graph kernels such as [77-80] have been proposed and widely adopted in many application areas (e.g, bio- and chemo-informatics [81-84]). Some of them support explicit feature vector representations of graphs (e.g, $[77,78,80])$. Thus both the aforementioned challenges C1.1 and C1.2 are effectively addressed by these graph kernels. Therefore, it just suffices to use a graph kernel together with a kernelized ML classifier (e.g., SVM) and we have a scalable, effective and ready-to-use malware detector. Recently, three approaches [32], [38] and [64], have successfully demonstrated using these general purpose graph kernels for malware detection.

Research Gap. However, a major problem in using these general purpose graph kernels on PRGs is that, they are not designed to take domain- and problem-specific observations into account. For instance, recent research on malware analysis has revealed that besides capturing neighbourhood (i.e., structural) information from PRGs it is important to capture the context under which the neighbourhoods are reachable to accurately detect malicious neighbourhoods $[28,29]$ (explained in detail in §3.2). Many existing graph kernels such as [77] and [78] can capture and compare structural information from PRGs effectively. However, they are not designed to capture the reachability context, as it is a strong domainspecific requirement and hence fail to do so. To address this, we develop a novel graph kernel which is capable of capturing both the aforementioned types of information.

For similar domain-specific reasons, researchers from other fields such as computer vision [84], bio- and chemo-informatics [81-83] have developed a number of kernels that specifically suit their applications. Despite graphs being natural representations of pro- 
grams and amenable for various activities, the program analysis research community has not devoted significant attention to development of domain-specific graph kernels. We take the first step towards this, by developing a kernel on PRGs which specifically suits our task of malware detection.

Our approach. To improve the accuracy of malware detection process, we propose a method to enrich the feature space of a graph kernel that inherently captures structural information with contextual information. We apply this feature-enrichment idea on a stateof-the-art graph kernel, namely, WLK [77] to obtain it contextual variant, named, CWLK. Specifically, CWLK associates to each sub-structure feature of WLK a piece of information about the context under which the sub-structure is reachable in the course of execution of the program. A sub-structure appearing in two different PRGs will match only if it is reachable under the same context in both PRGs. We show that for the malware detection problem, CWLK is more expressive and hence more accurate than WLK and other stateof-the-art kernels while maintaining comparable efficiency.

Experiments. Through our large-scale experiments with more than 50,000 Android apps, we demonstrate that CWLK outperforms two state-of-the-art graph kernels (incl. WLK) and three malware detection techniques by more than $5.27 \%$ and $4.87 \%$ F-measure, respectively, while maintaining high efficiency. This, in essence shows the significance of incorporating the contextual information along with structural information in the graph kernel while performing malware detection.

Contributions. In this chapter, we make the following contributions:

- We develop a graph kernel that captures both structural and contextual information from PRGs to perform accurate and scalable malware detection (§3.4). To the best of our knowledge, this is the first graph kernel specifically addressing a problem from the field of program analysis.

- Through large-scale experiments and comparative analysis, we show that the pro- 


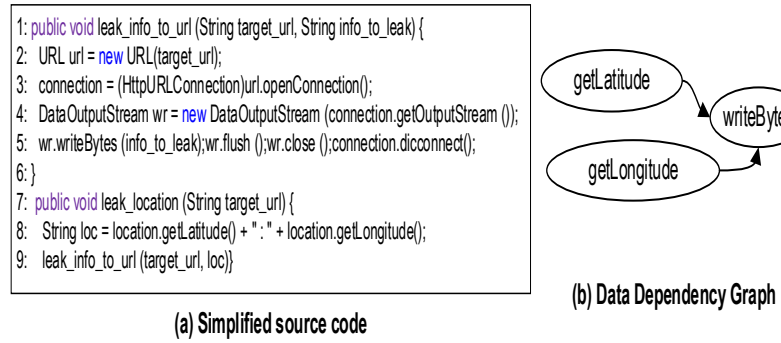

(a) Simplified source code

(b) Data Dependency Graph
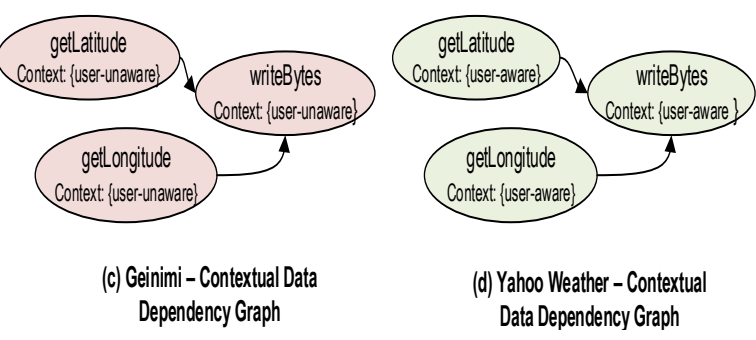

Figure 3.1: Location information being leaked in Geinimi (malware) and Yahoo Weather (benign) apps. (a) code snippet corresponding to leaking location information in both the apps. (b) DDG corresponding to the location leak. (c) Geinimi's DDG illustrating that it leaks information without the user's knowledge. (d) Yahoo Weather's DDG illustrating that it leaks information with the user's knowledge.

posed kernel outperforms two state-of-the-art graph kernels and three malware detection solutions in terms of accuracy, while maintaining high efficiency ( 33.5$)$.

- We make an efficient implementation of the proposed kernel (along with the dataset information) publicly available at: https://sites.google.com/site/cwlkernel.

\subsection{Motivation}

In this section, we motivate the design of our kernel by describing why considering just the structural information from PRGs is insufficient to determine the maliciousness of a sample and how supplementing it with contextual information helps to increase detection accuracy. To this end we use a real-world Android malware ${ }^{1}$ from the Geinimi family which steals users' private information. We contrast its behavior with that of a well-known benign app, Yahoo Weather.

Geinimi's execution. The app is launched through a background event such as receiving a SMS or call. Once launched, it reads the user's personal information such as geographic location and contacts and leaks the same to a remote server. The (simplified) malicious code portion pertaining to the location information leak is shown in Fig. 3.1

\footnotetext{
${ }^{1}$ SHA256:05620032f3a2abd5ebea482b5e5d5b8ff5faa8115019736013d87f442032b6bc
} 
(a). The method leak_location reads the geographic location through getLatitude and getLongitude APIs. Subsequently, it calls leak_info_to_url method to leak the location details (through DataOutputStream.writeBytes) to a specific server. The Data Dependency Graph (DDG) corresponding to the code snippet is shown in Fig. 3.1 (b). The nodes in DDG are labeled with the sensitive APIs that they invoke.

Yahoo Weather's execution. On the other hand, Yahoo Weather could be launched only by user's interaction with the device (e.g., by clicking the app's icon on the dash board). The app then reads the user's location and sends the same to its weather server to retrieve location-specific weather predictions. Hence, DDG portions of Yahoo Weather is same as that of Geinimi.

Contextual information. From the explanations above, it is clear that both the apps leak the same information in the same fashion. However, what makes Geinimi malicious is the fact that its leak happens without the user's consent. In other words, unlike Yahoo Weather, Geinimi leaks private information through an event which is not triggered by user's interaction. We refer to this as a leak happening in user-unaware context. On the same lines, we refer to Yahoo Weather's leak as happening in user-aware context.

As explained in [29] and [28], in the case of Android apps, one could determine whether a PRG node is reachable under user-aware or user-unaware context by examining its entry point nodes (i.e., nodes in PRGs that do not have any incoming edge, meaning, these nodes are invoked by Android framework level actions). Following this procedure we add the context as an attribute to every DDG node. This context annotated DDG of Geinimi and Yahoo Weather are shown in Fig. 3.1 (c) and (d), respectively.

Requirements for effective detection. From the aforementioned example the two key requirements that makes a malware detection process effective can be identified:

(R1) Capturing structural information. Since malicious behaviors often span across multiple nodes in PRGs, just considering individual nodes (and their attributes) in isolation 
is not enough. Capturing the structural (i.e., neighborhood) information from PRGs is of paramount importance.

(R2) Capturing contextual information. Considering just the structural information without the context is not enough to determine whether a sensitive behavior is triggered with or without user's knowledge. For instance, if structural information alone is considered, the features of both Geinimi and Yahoo Weather apps become identical, thus making the latter a false positive. Hence, it is important for the detection process to capture the contextual information as well to make the detection process more accurate.

Many existing graph kernels could address the first requirement well. However, the second requirement which is more domain-specific makes the problem particularly challenging. To the best of our knowledge, none of the existing graph kernels support capturing this reachability context information along with structural information. Hence, this gives us a clear motivation to develop a new kernel that specifically addresses our two-fold requirement.

\subsection{Definitions and Notations}

The formal definitions and notations that will be used throughout this chapter are presented in this section.

Definition 1.1 (Program Representation Graph). A PRG $G=(N, E, \lambda, \xi)$ is a directed graph where $N$ is a set of nodes and each node $n \in N$ denotes program entity such as a function or instruction. $E \subseteq(N \times N)$ is a set of edges and each edge $e\left(n_{1}, n_{2}\right) \in E$ denotes either control- or data-flow or dependency from $n_{1}$ to $n_{2} . \lambda$ is the set of labels that characterize the (security-sensitive) operations of a node and $\ell: N \rightarrow \lambda$, is a labeling function which assigns a label to each node. $\xi$ is a set of events that denote the context of a node and $C: N \rightarrow \xi$, is a function which assigns the context to each node.

Definition 1.2 (Context). The context of a node $n \in N$ in the PRG of a program $P$ is a 
set of attributes $\xi(n)$ that govern the reachability of $n$ in the course of execution of $P$.

Examples of contexts. In the case of Windows executables, the guard conditions that govern the execution of a node could be considered as its context [63]. Unlike Windows (and other desktop OS) binaries, Android and iOS mobile apps typically have multiple entry points $[28,29]$. Hence, in the case of such mobile apps, besides guard conditions, the categories of entry points through which a node is reachable could also be considered as its context. Similar platform-specific constraints and observations could be considered while defining the contexts for executables of other platforms.

\subsection{Contextual Weisfeiler-Lehman Graph Kernel}

In this section, We begin by explaining the regular WLK, then introduce the proposed CWLK and finally discuss how WLK falls short when performing malware detection using CADGs and how CWLK rectifies the same. Finally, we prove CWLK's semi-definitiveness and analyze its time complexity.

\subsubsection{Regular Weisfeiler-Lehman Graph Kernel}

WLK computes the similarities between graphs based on the 1-dimensional WL test of graph isomorphism [77].

WL test of isomorphism. Suppose we are to determine whether a given a pair of graphs $G$ and $G^{\prime}$ are isomorphic. The WL test of isomorphism works by augmenting the node labels by the sorted set of labels of neighboring nodes. This process is referred to as labelenrichment and new labels are referred as neighborhood labels. Thus, in each iteration $i$ of the WL algorithm, for each node $n \in N$, we get a new neighborhood label, $\lambda_{i}(n)$ that encompass the $i^{\text {th }}$ degree neighborhood around $n . \lambda_{i}(n)$ could be optionally compressed using a hash function $f_{c}: \Sigma^{*} \rightarrow \Sigma$ such that $f_{c}\left(\lambda_{i}(n)\right)=f_{c}\left(\lambda_{i}\left(n^{\prime}\right)\right)$, iff $\lambda_{i}(n)=\lambda_{i}\left(n^{\prime}\right)$. To 
test graph isomorphism, the re-labeling process is repeated until the neighborhood label sets of $G$ and $G^{\prime}$ differ, or the number of iterations reaches a specific threshold. Therefore, one iteration of WL relabeling is equivalent to a function $r\left(\left(N, E, \lambda_{i}\right)\right)=\left(N, E, \lambda_{i+1}\right)$ that transforms all graphs in the same manner.

Definition 1.3 (WL sequence). Define the WL graph at height $i$ of the graph $G=$ $(N, E, \lambda)$ as the graph $\mathscr{G}_{i}=\left(V, E, \lambda_{i}\right)$. The sequence of graphs

$$
\mathscr{G}_{0}, \mathscr{G}_{1}, \ldots, \mathscr{G}_{h}=\left(V, E, \lambda_{0}\right),\left(V, E, \lambda_{1}\right), \ldots,\left(V, E, \lambda_{h}\right)
$$

is called the WL sequence up to height $h$ of $G$, where $\mathscr{G}_{0}=G$ (i.e., $\lambda_{0}=\lambda$ ) is the original graph and $\mathscr{G}_{1}=r\left(\mathscr{G}_{0}\right)$ is the graph resulting from the first relabeling, and so on.

Definition 1.4 (WL kernel). Given a valid kernel $k(.,$.$) and the WL sequence of graph$ of a pair of graphs $G$ and $G^{\prime}$, the WL graph kernel with $h$ iterations is defined as

$$
k_{W L}^{(h)}\left(G, G^{\prime}\right)=k\left(\mathscr{G}_{0}, \mathscr{G}_{0}^{\prime}\right)+\ldots+k\left(\mathscr{G}_{h}, \mathscr{G}_{h}^{\prime}\right)
$$

where $h$ is the number of WL iterations and $\mathscr{G}_{0}, \mathscr{G}_{1}, \ldots, \mathscr{G}_{h}$ and $\mathscr{G}_{0}^{\prime}, \mathscr{G}_{1}^{\prime}, \ldots, \mathscr{G}_{h}^{\prime}$ are the WL sequences of $G$ and $G^{\prime}$, respectively. $h$ is referred as height of the kernel.

Intuitively, WLK counts the common neighborhood labels in two graphs. Hence we have $k_{W L}^{(h)}\left(G, G^{\prime}\right)=\left|\left(\lambda_{i}(n), \lambda_{i}\left(n^{\prime}\right)\right)\right|$, iff $f_{c}\left(\lambda_{i}(n)\right)=f_{c}\left(\lambda_{i}\left(n^{\prime}\right)\right)$ for $i \in\{0, \ldots, h\}, n \in N, n^{\prime} \in N^{\prime}$, where $f_{c}$ is injective and the sets $\left\{f_{c}\left(\lambda_{i}(n)\right) \mid n \in N \cup N^{\prime}\right\}$ and $\left\{f_{c}\left(\lambda_{j}(n)\right) \mid n \in N \cup N^{\prime}\right\}$ are disjoint for all $i \neq j$.

\subsubsection{Contextual Weisfeiler-Lehman Graph Kernel}

The goal of CWLK is to capture not only neighborhoods around the node, but also to include the contexts in which each of the neighborhoods is reachable in the PRG. To this 


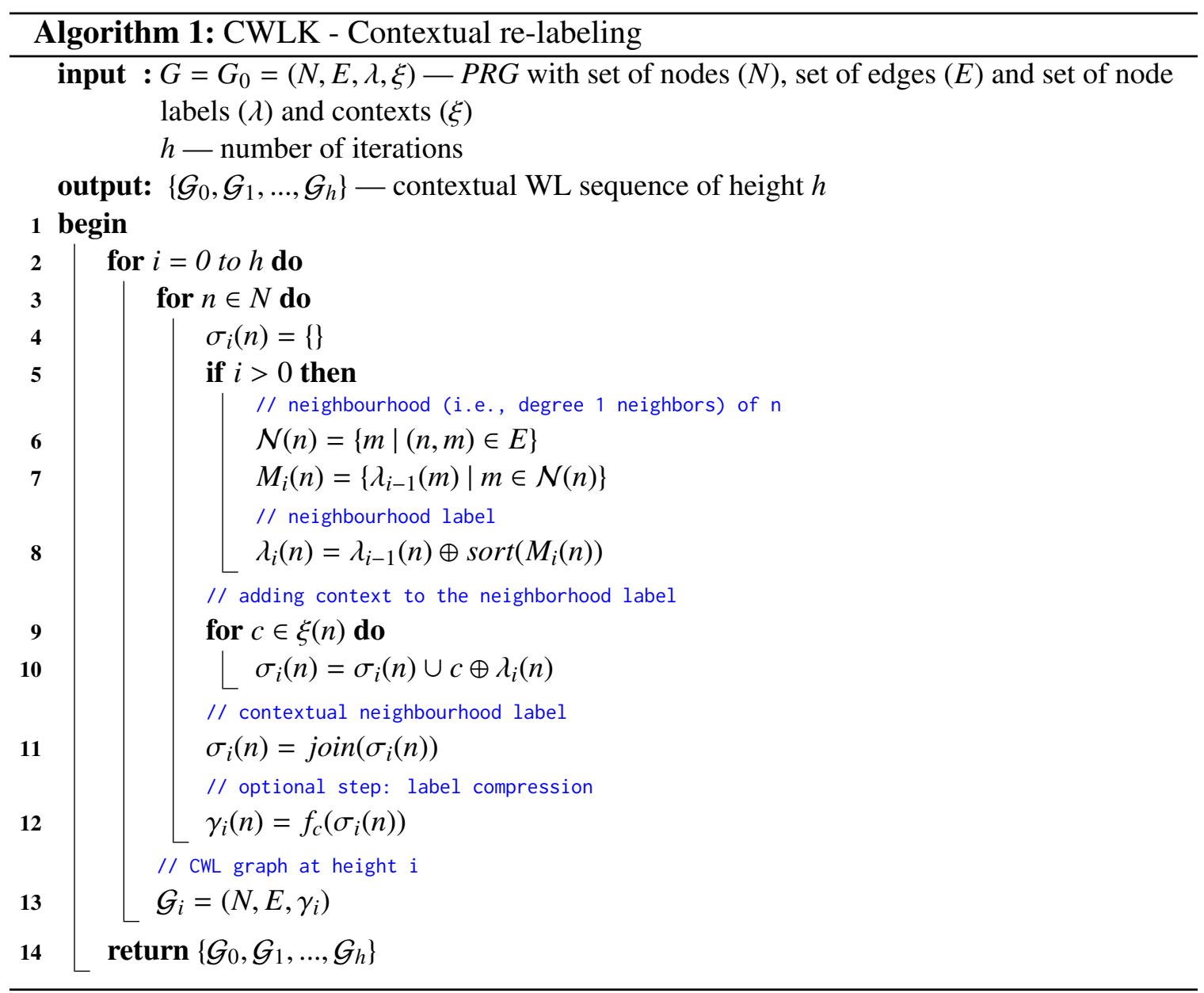

end, we modify the re-labeling step of WLK so as to accommodate the context of every neighborhood. We refer to this process as contextual-relabeling and the sequence of graphs thus obtained as contextual WL sequence.

Contextual re-labeling. Specifically, CWLK performs one additional step in the relabeling process which is to attach the contexts of every node to its neighborhood label in every iteration. This in effect, indicates the contexts under which a particular neighborhood is reachable. The label thus obtained is referred to as contextual neighborhood label. The contextual relabeling process is presented in detail in Algorithm 1.

The inputs to the algorithm are PRG, $G$ and the degree of neighbourhoods to be considered for re-labeling, $h$. The output is the sequence of contextual WL graphs, $\left\{\mathcal{G}_{0}, \mathcal{G}_{1}, \ldots, \mathcal{G}_{h}\right\}=$ 
$\left\{\left(N, E, \gamma_{0}\right),\left(N, E, \gamma_{1}\right), \ldots,\left(N, E, \gamma_{h}\right)\right\}$, where $\gamma_{0}, \ldots, \gamma_{h}$ are constructed using the contextual relabeling procedure.

For the initial iteration $i=0$, no neighborhood information needs to be considered. Hence the contextual neighborhood label $\gamma_{0}(n)$ for all nodes $n \in N$ is obtained by justing prefixing the contexts to the original node labels and compressing the same (lines 9-12). For $i>0$, the following procedure is used for contextual re-labeling. Firstly, for a node $n \in N$, all of its neighboring nodes are obtained and stored in $\mathcal{N}(n)$ (line 6). For each node $m \in \mathcal{N}(n)$ the neighborhood label up to degree $i-1$ is obtained and stored in multiset $M_{i}(n)$ (line 7). $\lambda_{i-1}(n)$, neighborhood label of $n$ till degree $i-1$ is concatenated to the sorted value of $M_{i}(n)$ to obtain the current neighborhood label, $\lambda_{i}(n)$ (line 8$)$. Finally the current neighborhood label is prefixed with the contexts of node $n$ to obtain the string $\sigma_{i}(n)$ which is then compressed using the function $f_{c}$ to obtain the contextual neighborhood label, $\gamma_{i}(n)$ (lines 9-12). For every iteration $i$, this process of contextual relabeling yields CWL graph at height $i, \mathcal{G}_{i}$ (line 13). Finally, the CWL sequence comprising CWL graphs from height 0 to $h$ are returned (line 14).

Definition 1.5 (CWL kernel). Given a valid kernel $k(.,$.$) and the CWL sequence of$ graph of a pair of graphs $G$ and $G^{\prime}$, the contextual WL graph kernel with $h$ iterations is defined as

$$
k_{W L}^{(h)}\left(G, G^{\prime}\right)=k\left(\mathcal{G}_{0}, \mathcal{G}_{0}^{\prime}\right)+\ldots+k\left(\mathcal{G}_{h}, \mathcal{G}_{h}^{\prime}\right)
$$

where $h$ is the number of CWL iterations and $\mathcal{G}_{0}, \mathcal{G}_{1}, \ldots, \mathcal{G}_{h}$ and $\mathcal{G}_{0}^{\prime}, \mathcal{G}_{1}^{\prime}, \ldots, \mathcal{G}_{h}^{\prime}$ are the CWL sequences of $G$ and $G^{\prime}$, respectively.

Intuitively, CWLK counts the common contextual neighborhood labels in two graphs. Hence we have $k_{C W L}^{(h)}\left(G, G^{\prime}\right)=\left|\left(\sigma_{i}(n), \sigma_{i}\left(n^{\prime}\right)\right)\right|$, iff $f_{c}\left(\sigma_{i}(n)\right)=f_{c}\left(\sigma_{i}\left(n^{\prime}\right)\right)$ for $i \in\{0, \ldots, h\}, n \in$ $N, n^{\prime} \in N^{\prime}$.

Illustrating WLK's shortcoming and CWLK's efficacy. Having presented the formulations for both kernels, we now illustrate WLK's shortcomings and explain how CWLK 


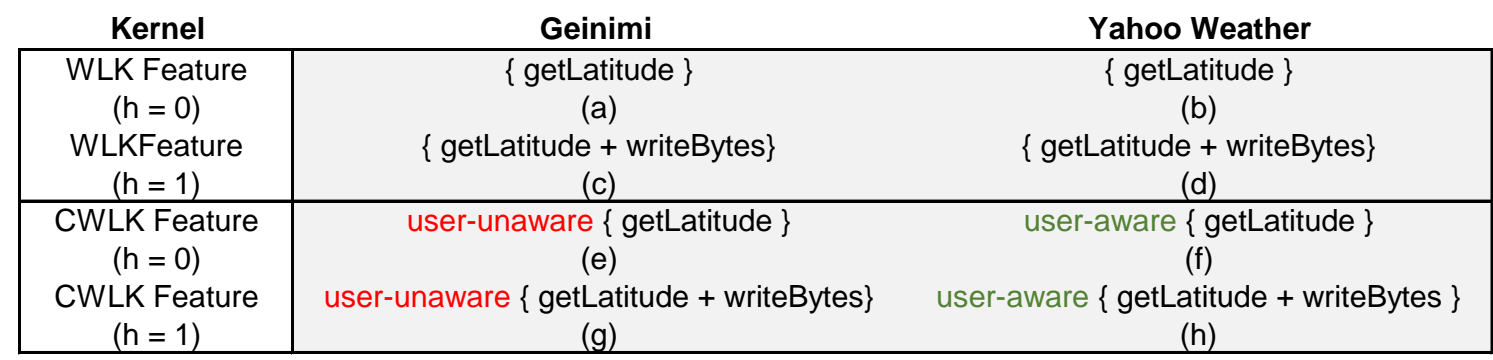

Figure 3.2: WLK and CWLK neighborhood labels for the node getLati tude from Geinimi and Yahoo Weather apps.

addresses the same, with an example. Applying WLK on the CADGs of Geinimi and Yahoo Weather examples (see Fig. 3.1 (c) and (d)), for the node getLatitude, for heights $h=0,1$, we get the neighborhood labels as shown in Fig. 3.2 (a)-(d). In both cases, the node getLatitude has only one degree-1 neighbor, writeBytes and this fact is reflected in the neighborhood label. Clearly, WLK captures the structural information around the node getLatitude, incrementally in every iteration of $h$. In fact, neighborhood label for $h=1$ captures that a sensitive node, wri teBytes lies in the neighborhood of getLati tude, which highlights a possible privacy leak. However, WLK does not capture whether the neighborhood involved in this leak is reached in user-aware or unaware context. Hence, whether or not the leak is malicious still remains inconclusive. This is precisely what CWLK addresses. On applying CWLK for the same node in both apps, we obtain the contextual neighborhood labels as shown in Fig. 3.2 (e)-(h). Clearly, the contextual neighborhood labels of Geinimi reveal that the sensitive operations are performed in the userunaware context, whereas, the same for Yahoo Weather happen in the user-aware context. Hence, it is evident that the CWLK's contextual relabeling provides a means to clearly distinguish malicious CADG neighborhoods from the benign ones. Therefore, unlike WLK, CWLK based classification does not detect Yahoo Weather as a false positive. This example clearly establishes the suitability of CWLK for the malware detection task.

We now prove CWLK's positive definiteness and also analyze its time complexity.

Theorem 1. CWLK is positive definite. 
Proof. Let us define a mapping $\phi$ that counts the occurrences of a particular contextual neighborhood label sequence $\sigma$ in $G$ (generated in $h$ iterations of Algorithm 1). Let $\phi_{\sigma}^{(h)}(G)$ denote the number of occurrences of $\sigma$ in $G$, and analogously $\phi_{\sigma}^{(h)}\left(G^{\prime}\right)$ for $G^{\prime}$. Then,

$$
\begin{array}{r}
k_{\sigma}^{(h)}\left(G, G^{\prime}\right)=\phi_{\sigma}^{(h)}(G), \phi_{\sigma}^{(h)}\left(G^{\prime}\right)=\mid\left\{\left(\sigma_{i}(n), \sigma_{i}\left(n^{\prime}\right)\right) \mid\right. \\
\left.\sigma_{i}(n)=\sigma_{i}\left(n^{\prime}\right), i \in\{0, \ldots, h\}, n \in N, n^{\prime} \in N^{\prime}\right\} \mid
\end{array}
$$

Summing over all $\sigma$ from the vocabulary $\Sigma^{*}$, we get

$$
\begin{aligned}
& k_{C W L}^{(h)}\left(G, G^{\prime}\right)= \sum_{\sigma \in \Sigma^{*}} k_{\sigma}^{(h)}\left(G, G^{\prime}\right)=\sum_{\sigma \in \Sigma^{*}} \phi_{\sigma}^{(h)}(G) \phi_{\sigma}^{(h)}\left(G^{\prime}\right) \\
&=\left|\left\{\left(\sigma_{i}(n), \sigma_{i}\left(n^{\prime}\right)\right) \mid \sigma_{i}(n)=\sigma_{i}\left(n^{\prime}\right), i \in\{0, \ldots, h\}, n \in N, n^{\prime} \in N^{\prime}\right\}\right| \\
&=\mid\left\{\left(\sigma_{i}(n), \sigma_{i}\left(n^{\prime}\right)\right) \mid f_{c}\left(\sigma_{i}(n)\right)=f_{c}\left(\sigma_{i}\left(n^{\prime}\right)\right),\right. \\
&\left.\quad i \in\{0, \ldots, h\}, n \in N, n^{\prime} \in N^{\prime}\right\} \mid
\end{aligned}
$$

where the last equality follows from the fact that $f_{c}$ is injective.

As $f_{c}(\sigma) \neq f_{c}\left(\sigma^{\prime}\right)$ if $\sigma \neq \sigma^{\prime}$, the string $\sigma$ corresponds to exactly one contextual neighborhood label and $k_{C W L}^{(h)}$ defines a kernel with corresponding feature map $\phi_{C W L}^{(h)}$, such that

$$
\phi_{C W L}^{(h)}=\left(\phi_{\sigma}^{(h)}(G)\right)_{\sigma \in \Sigma^{*}}
$$

Complexity. The runtime complexity of CWLK with $h$ iterations on a graph with $n$ nodes and $e$ edges is $O(h e$ ) (assuming that $e>n$ ) which is same as that of WLK. More specifically, the neighborhood label computation with sorting operations (lines 6-8 of Algorithm 1) take $O(e)$ time for one iteration and the same for $h$ iterations take $O(h e)$. The inclusion of context (lines 9 and 10), does not incur additional overhead as $e>>|\xi|$. Hence the final time complexity remains as $O(h e)$. For a detailed derivation and analysis of the time complexity of WLK, we refer the reader to [77]. 
Table 3.1: Composition of dataset

\begin{tabular}{|c|c|c|}
\hline Portion of dataset & Dataset source & \# of samples \\
\hline \hline Malware portion & DREBIN [25], Virus Share [14] & 29877 \\
Benign portion & Google Play [4] & 25000 \\
\hline
\end{tabular}

Efficient computation of CWLK on K graphs. When computing CWLK on $K$ graphs to obtain $K \times K$ kernel matrix, a naïve approach would involve $K^{2}$ comparisons, resulting a time complexity of $O\left(K^{2} h e\right)$. However, as mentioned in [77], a Bag-of-Features (BoF) model based optimization could be performed to arrive the kernel matrix in $O\left(K h e+K^{2} h n\right)$ time. This optimized computation involves the following steps: (1) A vocabulary $\Sigma$ of all the contextual neighbourhood labels of nodes across the $K$ graphs is obtained in $O(K h e)$ time. This facilitates representing each of the $K$ graphs as feature vectors of $|\Sigma|$ dimensions. (2) Subsequently, $K \times K$ kernel matrix can be computed by multiplying these vectors in $O\left(K^{2} h n\right)$ time.

In summary, CWLK has the same efficiency as that of WLK and supports explicit feature vector representations of PRGs.

Relation to other spatial contextual kernels. Two recently proposed graph kernels [80] and [79], consider incorporating the spatial context information to neighborhood subgraph features. They define context of a subgraph feature as another subgraph appearing in its vicinity. As mentioned earlier, in our malware detection problem we refer to attributes of a node which determine its reachability as its context. This reachability context is different from spatial context discussed in [80] and [79]. Hence CWLK is consummately different from these two kernels.

\subsection{Evaluation}

We conducted large scale experiments involving more than 50,000 Android apps from two real-world malware datasets to evaluate the accuracy and efficiency of CWLK. We com- 
Table 3.2: Dataset Statistics

\begin{tabular}{|l|l|l|l|}
\hline \multicolumn{2}{|c|}{ CG (Avg \pm Std) } & \multicolumn{2}{c|}{ ICFG (Avg \pm Std) } \\
\hline \hline \# of Nodes & \# of Edges & \# of Nodes & \# of Edges \\
\hline $1556 \pm 998$ & $3327 \pm 1803$ & $15323 \pm 9844$ & $22745 \pm 20922$ \\
\hline
\end{tabular}

pare CWLK's performance against that of two state-of-the-art kernels and three Android malware detection solutions.

\subsubsection{Datasets}

DrEBIN [25] provides a collection on 5,560 Android malware apps collected from 2010 to 2012. More recently, Virus-share [14] released a collection of 24,317 malware apps collected from 2010 to 2014. We combined these two datasets and use them in our evaluation. For the benign portion of the dataset, we collected 25,000 benign top-selling apps from Google Play store [4] that were released around the same time. Thus, our dataset contains a total of 54,877 apps. The composition of our dataset is presented in Table 3.1.

Graph Representations considered. As mentioned in $\S 3.1$ the proposed CWLK could be applied on any type of PRGs to perform malware detection. In our evaluations, we experimented with two types of PRGs namely, CGs and inter-procedural control-flow graphs (ICFGs). The nodes of a CG represent the methods present in an app; its directed edges represent the calling/called relations between the methods. The nodes of an ICFG represent the individual instructions present in those methods; its edges represent the control-flows among those instructions. These two types of PRGs are chosen since they capture program semantics at different levels of granularities. The statistics on the average number of nodes and edges of the PRGs in our dataset are presented in Table 3.2. Details on construction of these PRGs are presented later in §3.5.2.

Training and Test sets. $60 \%$ of the samples were randomly chosen from the datasets and used for training the classifier and the remaining $40 \%$ samples are used to test their 
performances. The classifiers' hyper-parameters are determined on the training set (using a portion of it as validation set) using 5-fold cross-validation, whereas the test set is only used for determining the final detection performance. We repeat this procedure 5 times and average the results.

\subsubsection{Implementation \& Comparative Analysis}

PRG construction \& Context Identification. Both CG and ICFG of the apps in our datasets are constructed through static analysis using Soot [17], a well-known Android static analysis workbench. The nodes are labeled with the security-sensitive APIs they access and are annotated with context information. Nodes that do not access any sensitive APIs are removed. We use the category of entry points of each of the nodes in these graphs as their contexts. The procedure proposed in DroidSIFT [29] is used to identify and categorize each entry point as being in 'user-aware' or 'user-unaware' context.

Comparison with Graph Kernels. CWLK's accuracy and efficiency is compared against those of WLK [77] and NHGK [78]. Since these kernels cannot capture context information, we use the comparative analysis against them to ascertain whether including context information significantly improves the accuracy without affecting the efficiency. We implemented all these kernels in about 2,170 lines of Python code. For all the kernels, BoF model based implementation similar to the one discussed in $§ 3.4 .2$ is used obtain explicit feature vector representation of samples.

Comparison with Malware Detection Solutions. Also, our approach is compared against three light-weight state-of-the-art ML based Android malware detection solutions, namely, Drebin [25], Allix et al. [26] and Adagio [32]. To this end, we re-implemented DrEBIN and Allix et al.'s approaches through consultations with the authors. For AdAGIO, an open-source implementation provided by the authors is used. 
Since the malware detection accuracy of these solutions predominantly depend on the features they use, we briefly introduce them here. DREBIN [25] considers features such as sensitive Android APIs, permissions and components used by apps. Allix et al. [26] constructs the CFGs of individual methods and represent them as signature strings which are subsequently used as features. Adagio [32] constructs CGs and uses byte-code instructions to assign labels to nodes. NHGK [78] is used to extract CG neighborhoods as features and a HI kernel SVM is trained to detect malware. Due to severe scalability issues (explained in §3.5.4) AdAGIo is ran only once on our dataset. All other techniques are ran 5 times and the average results are reported.

\subsubsection{Experimental Design}

Research Questions (RQs). Through our evaluations, we seek to address the following RQs:

(RQ1) Does including the context information in PRGs through CWLK significantly improve the malware detection accuracy?

(RQ2) Does capturing context information incur significant computation overhead to adversely affect the efficiency?

(RQ3) Does our context-based detection approach outperform existing malware detection solutions?

Two separate experiments are designed to address these questions.

Experiment E1. In order to evaluate the first two questions, the following experiment is conducted: First, the CGs of all the training set apps are constructed. Then, the WLK, NHGK and CWLK kernels are applied on the CGs to obtain respective kernel matrices. Subsequently, a SVM classifier is trained with each of these kernels to detect malicious apps. Finally, the test set apps are subjected to the aforementioned kernel computation processes and are used to evaluate the models. The same procedure is repeated with ICFGs. 


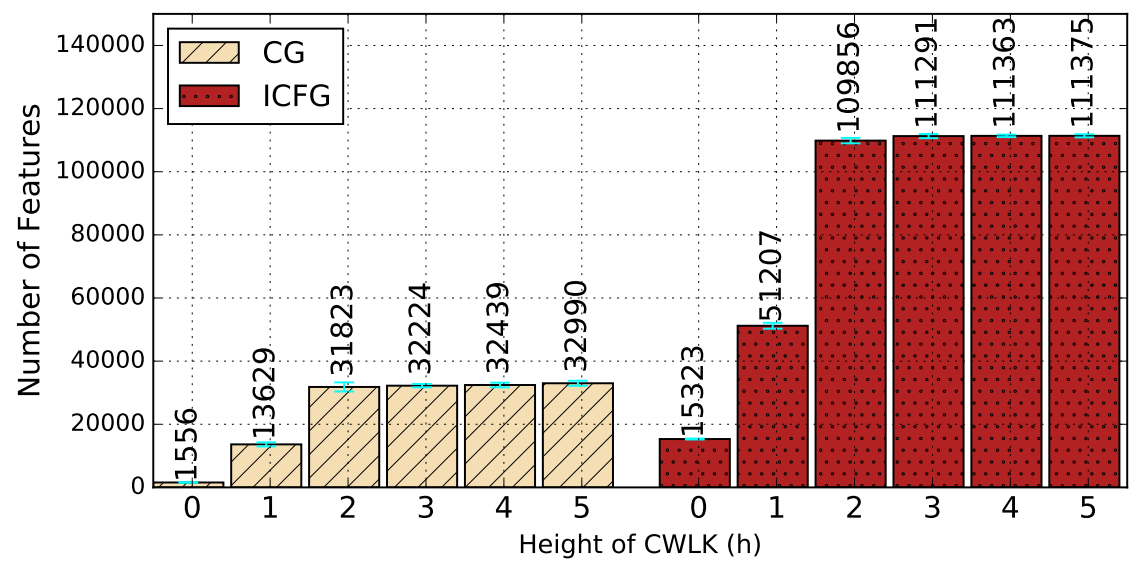

Figure 3.3: No. of features obtained for various heights of CWLK.

Experiment E2. In order to address question (3), we subject Drebin [25], Allix et al. [26] and Adagio [32] solutions to the same training and test sets. We compare them against the best performing model that uses CWLK (obtained from experiment E1).

Setting the parameter $\boldsymbol{h}$ : We experimented with different kernel heights $h=0$ to 5 for CWLK (see eq. (3.3)). The average number of contextual neighborhood features (from 5 runs of E1) for different values of $h$ on the two PRGs is reported in Fig. 3.3. It is evident that the number of features does not increase significantly after $h=2$ on both PRGs. This is because we have removed nodes that do not access sensitive APIs which affects the connectivity and restricts the neighborhood sizes. In other words, we seldom have neighborhoods around nodes that span beyond degree 2. Similar trend is observed in WLK and NHGK features. Hence we restrict the height $h$ to be 0,1 and 2 for all three kernels. Thus, for each kernel applied on each PRG, we have three SVM classifiers (one for each $h$ ). Therefore we have a total of 18 malware detection models under comparison (see Table 3.3 for details).

Evaluation metrics. Standard evaluation metrics such as Precision, Recall and Fmeasure are used to determine the effectiveness of malware detection [26]. All these values are expressed as percentages and higher values indicate accurate detection. Efficiency is determined in terms of training and testing durations. Lower values of these durations indicate 
scalable detection.

Evaluation Setup. All the experiments were conducted on Intel Xeon Hexa-core E52640 processor $(2.50 \mathrm{GHz})$ with $32 \mathrm{~GB}$ RAM running Ubuntu 14.04.

\subsubsection{Results and Discussions}

\section{RQ1: Impact of context information on F-measure}

We compare the Precision, Recall and F-measure of the malware detection process through CWLK with those of WLK and NHGK. This is to ascertain whether incorporating the context information in PRGs boosts the effectiveness of detection. These results for the 18 models are presented in Table 3.3, from which the following inferences are drawn:

- At the outset, two general observations are made: (1) All models perform better on ICFGs compared to CGs. This is because, ICFG is a more fine-grained representation of programs than $\mathrm{CG}$, which enables it to capture program semantics at a finer level and thereby boosting the detecting accuracy. Hence, we conclude that in our experiments ICFG is a more effective representation for malware detection. (2) Considering larger neighborhoods helps capturing the structural information better which in turn reflects in better performances. This is evident as Precision, Recall and F-measure values get better with increasing values of $h$ for all the three kernels. However, this observation may not hold for large values of $h$, as nodes that are far apart will be considered for neighborhood re-labeling, leading to a noisy re-labeling process.

- It is clear that CWLK outperforms both WLK and NHGK in terms of F-measure consistently on both CG and ICFG representations.

- Since the only difference between WLK and CWLK is the latter's capability to capture the context information, evidently this is the reason for CWLK's superior performance.

- Also, CWLK achieves better Precision than WLK in all the experiments, consistently. 
Table 3.3: Comparison of CWLK against state-of-the-art Graph Kernels (metrics expressed as \% values) - avg. over 5 runs

\begin{tabular}{|c|c|c|c|c|c|c|c|c|c|c|}
\hline \multirow{2}{*}{$\begin{array}{l}\text { PRG } \\
\text { Type }\end{array}$} & \multirow{2}{*}{ Kernel } & \multicolumn{3}{|c|}{$h=0$} & \multicolumn{3}{|c|}{$h=1$} & \multicolumn{3}{|c|}{$h=2$} \\
\hline & & Precision & Recall & F-measure & Precision & Recall & F-measure & Precision & Recall & F-measure \\
\hline \multirow{3}{*}{ CG } & $\overline{~ W L K ~[77] ~}$ & $71.21( \pm 1.15)$ & $\overline{880.93( \pm 1.78)}$ & $75.75( \pm 1.28)$ & $85.00( \pm 1.59)$ & $85.93( \pm 2.10)$ & $85.46( \pm 1.39)$ & $87.28( \pm 1.02)$ & $86.15( \pm 0.78)$ & $86.71( \pm 0.88)$ \\
\hline & NHGK [78] & $63.05( \pm 3.11)$ & $60.14( \pm 2.04)$ & $61.56( \pm 2.56)$ & $67.18( \pm 1.76)$ & $69.02( \pm 2.01)$ & $68.08( \pm 1.88)$ & $74.17( \pm 1.11)$ & $70.22( \pm 1.69)$ & $72.14( \pm 1.88)$ \\
\hline & CWLK & $77.29( \pm 1.75)$ & $79.85( \pm 0.83)$ & $78.54( \pm 0.97)$ & $88.50( \pm 0.95)$ & $87.98( \pm 1.41)$ & $88.23( \pm 1.10)$ & $89.19( \pm 0.83)$ & $\mathbf{9 0 . 1 0}( \pm 0.96)$ & $89.64( \pm 0.94)$ \\
\hline \multirow{3}{*}{ ICFG } & WLK [77] & $85.49( \pm 2.59)$ & $81.66( \pm 3.71)$ & $83.53( \pm 2.19)$ & $90.17( \pm 1.92)$ & $87.48( \pm 1.20)$ & $88.8( \pm 1.37)$ & $93.09( \pm 0.81)$ & $88.16( \pm 0.77)$ & $90.55( \pm 0.80)$ \\
\hline & NHGK [78] & $68.68( \pm 4.10)$ & $70.73( \pm 2.77)$ & $69.68( \pm 3.20)$ & $75.78( \pm 2.18)$ & $81.01( \pm 2.64)$ & $78.30( \pm 2.06)$ & $78.11( \pm 1.07)$ & $82.28( \pm 1.50)$ & $80.14( \pm 1.29)$ \\
\hline & CWLK & $89.74( \pm 2.33)$ & $81.25( \pm 3.12)$ & $85.28( \pm 2.59)$ & $96.77( \pm 1.63)$ & $\mathbf{9 2 . 0 8}( \pm 1.20)$ & $94.36( \pm 1.15)$ & $97.15( \pm 0.28)$ & $94.53( \pm 0.75)$ & $\mathbf{9 5 . 8 2}( \pm 0.66)$ \\
\hline
\end{tabular}


This indicates that CWLK suffers lesser false positives than WLK. This reduction in false positives is a direct result of capturing context information which helps to precisely distinguish malicious neighborhoods in the PRGs from the benign ones (as discussed in $\S 3.4)$.

- NHGK consistently fails to produce better results than WLK and CWLK. This is because, the node labeling and hashing technique adapted in NHGK causes collisions among informative and non-informative subgraph features. Such collisions could be avoided in WLK and CWLK through label compression (as explained in §3.4), making them more expressive and accurate. For further details on this limitation of NHGK, we refer the reader to the original work at [78] (particularly Section 5 where the authors discuss this limitation in detail).

Since CWLK uses both contextual and structural information, it is important to analyze the contribution of each of these types of information to its performance. Capturing only structural information is equivalent to using WLK. Hence from rows 1 and 4 of Table 3.3 (ignoring columns for $h=0$ ), it is evident that structural information alone could provide a minimum of $85.46 \%$ and an average of $87.88 \%$ F-measure across both PRGs. Similarly, the contribution of contextual information alone is ascertained using CWLK and setting $h=0$ to be a minimum of $78.54 \%$ and an average of $81.91 \%$ F-measure. Finally, the effect of using both types of information is ascertained by using CWLK and setting $h>0$ to be a minimum of $88.23 \%$ and an average of $92.02 \%$ F-measure across both PRGs. This clearly conveys that structural information is primary for performing effective malware detection and contextual information complements it, thereby helping to improve the accuracy. CWLK attains superior performance by capturing both these types of information.

Statistical significance test. Now, we intend to study whether the improvements in malware detection accuracy achieved by CWLK over WLK is statistically significant. To 

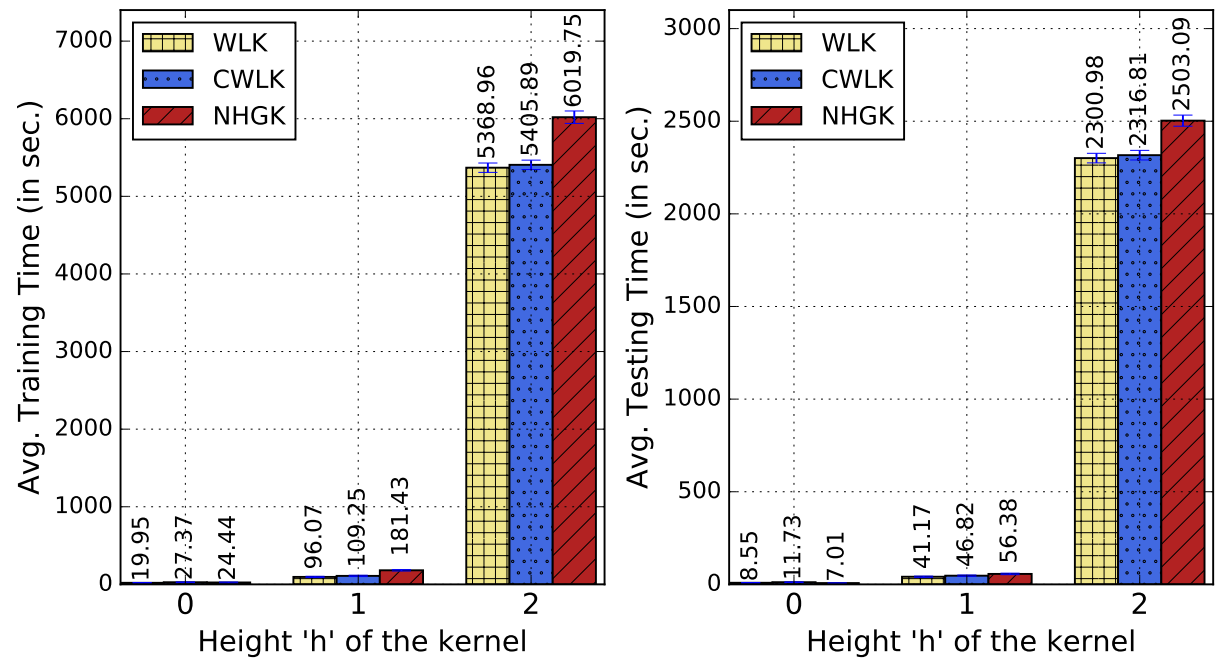

Figure 3.4: Training and Testing durations for different values of $h$ on ICFG representation (avg. over 5 runs)

this end, we compare their mean F-scores reported in Table 3.3 using a well-known nonparametric statistical significance test, namely, Wilcoxon signed rank test [134] and observe that the $\mathrm{p}$-value is 0.0277 . Since the $\mathrm{p}$-value is less than 0.05 , we conclude that CWLK statistically significantly improves WLK's malware detection accuracy with 95\% confidence.

\section{RQ2: Impact of context information on efficiency}

We compare the training and testing durations of classifiers using the three kernels under study from experiment E1. This is to infer whether CWLK achieves higher accuracy at the cost of higher computation time. As discussed earlier in $\S 3.4$, CWLK’s time complexity is same as that of WLK. Hence, we expect negligible difference in efficiency.

Experimental Setting. Due to space constraints, the training and testing times of the worst-case experimental setting involving samples with maximum number of nodes and edges are only reported. This setting corresponds to using ICFG representations of apps. The average training and testing durations in this experimental setting for various values of $h$ over 5 runs of experiment E1 are presented in Fig. 3.4, from which the following inferences are drawn. 
Table 3.4: Comparison of CWLK based malware detection against the state-of-the-art detectors (avg. over 5 runs)

\begin{tabular}{|c|c|c|c|}
\hline Method & Precision & Recall & F-measure \\
\hline \hline DREBIN [25] & $97.02( \pm 0.93)$ & $85.60( \pm 1.03)$ & $90.95( \pm 0.71)$ \\
Allix et al. [26] & $88.24( \pm 0.74)$ & $86.29( \pm 1.66)$ & $87.25( \pm 0.54)$ \\
ADAGIO [32] & 92.18 & 87.32 & 89.68 \\
CWLK & $\mathbf{9 7 . 1 5}( \pm \mathbf{0 . 2 8})$ & $\mathbf{9 4 . 5 3}( \pm \mathbf{0 . 7 5})$ & $\mathbf{9 5 . 8 2}( \pm \mathbf{0 . 6 6})$ \\
\hline
\end{tabular}

- The training and testing durations of CWLK and WLK are almost same for all values of $h$. This is due to the fact that adding the context information which is the only additional operation in CWLK results in negligible difference on the total kernel computation, training and testing durations.

- The training and testing durations increase with the height of the kernel $h$ for all the kernels in a similar fashion. This is mainly due to the fact that both WLK and CWLK condense the neighborhood labels in the same way which is very similar to that of NHGK. Also, for all these kernels, there is a huge increase in training and testing durations when $h$ increases from 1 to 2 which does not reflect proportionally in terms of F-measure. Hence, one may choose to perform malware detection in a much scalable and reasonably accurate manner by setting $h=1$ (i.e., considering only degree-1 neighbors).

- It is noted that CWLK along with the other two kernels in Fig. 3.4, shows high efficiency as it operates in linear time on the density of PRGs. In particular, it is more efficient than the classic walk-, tree- and path-based graph kernels (discussed in [99]) and is suitable for large-scale malware detection.

From experiment E1, we conclude that CWLK through its virtue of capturing both structural and contextual information, significantly improves upon the accuracy of WLK for the malware detection task without hurting the efficiency. 

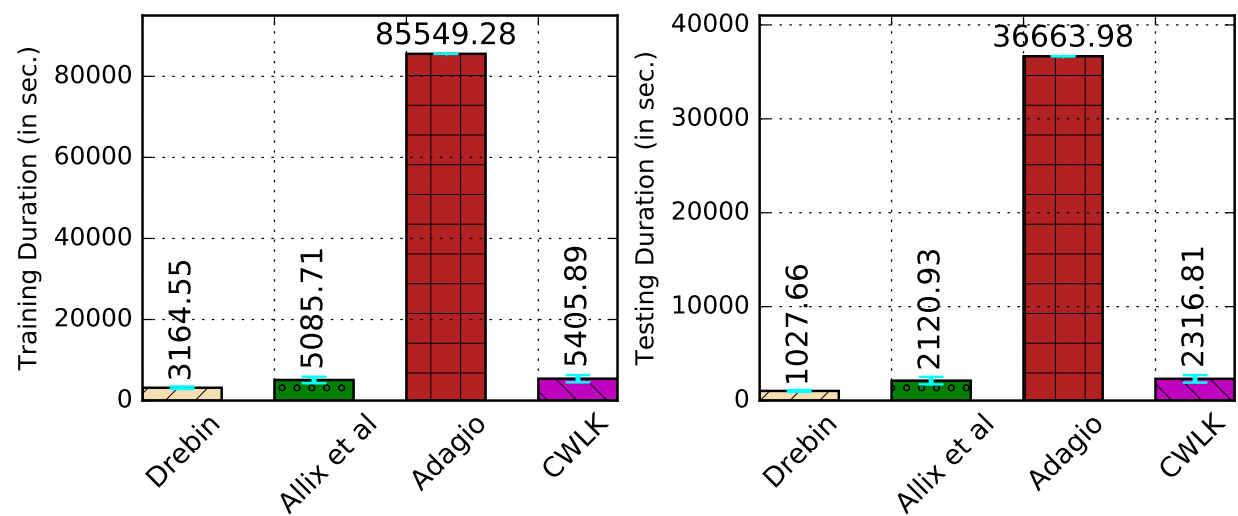

Figure 3.5: Comparison on Efficiency of CWLK with state-of-the-art malware detectors (avg. over 5 runs)

\section{RQ3: CWLK Vs. state-of-the-art malware detectors}

We now compare CWLK based detection with the state-of-the-art Android malware detection solutions to study whether contextual PRG neighborhoods makes good features for malware detection, through experiment E2. For CWLK based detection, ICFG representation with $h=2$ is used as it offers the best performance. The Precision, Recall and F-measures of each of these methods are reported in Table 3.4. The following observations are made from the table:

- Clearly, CWLK based malware detection outperforms all the compared solutions in terms of F-measure. In particular, our approach outperforms the best performing technique (i.e., Drebin) by 4.87\% F-measure. In terms of Precision, our approach outperforms AdAGio and Allix et al.'s methods and is comparable to DreBIN. In terms of Recall, ours outperforms other methods.

- Out of the methods compared, DreBin does not use both structural and contextual features. ADAGio and Allix et al.'s approaches use structural information but not contextual information. This reveals that capturing both these types of information is the reason for our approach's superior performance, reinforcing our findings from experiment E1. 
We now compare the efficiency of CWLK based detection against that of state-of-the-art malware detectors. It is noted that these techniques use different features and classifiers and hence a wide variation in training and testing durations is expected. The results of this comparison is presented in Fig. 3.5, from which the following observations are made:

- Drebin being a light-weight non PRG based approach it has significantly higher efficiency than all other methods, including ours.

- Allix et al.'s method is similar to ours in terms of using PRG based features. Hence our efficiency is comparable to this method.

- Adagio uses NHGK and HI kernel SVM in the primal formulation. Hence it takes a prohibitively long time for training and testing. Our method is far more efficient than Adagio.

In conclusion, our method's efficiency is comparable to that of other PRG based methods, far better than heavy-weight approaches and inferior to non PRG based light-weight methods.

From experiment E2, we conclude that when compared to state-of-the-art malware detectors, CWLK produces considerably higher accuracy with a practically tractable efficiency, making it suitable for large-scale real-world malware detection.

From experiment E2, we conclude that when compared to state-of-the-art malware detectors, CWLK produces considerably higher accuracy with a practically tractable efficiency, making it suitable for large-scale real-world malware detection.

\subsection{Related Work}

The related works on general purpose graph kernels (i.e., facilitating any graph classification application) and application-specific graph kernels are reviewed in the two following 
subsections.

\subsubsection{General Purpose Graph Kernels}

Several general purpose graph kernels have been proposed and used in many real-world applications. These graphs kernels can be categorized into four major families:

1. Graph kernels based on walks/paths. RW [74] and SP [76] graph kernels are classic examples of this family. The RW kernel counts the number of common walks when simultaneous walks are performed on a pair of graphs. The SP kernel simply compares the sorted endpoints and the length of shortest-paths that are common between two graphs. A significant bottleneck for both these kernels is their computational cost. The best known time complexity for exact computation is $O\left(n^{3}\right)$ for both RW and SP kernels. Attempts have been made to reduce the runtime. For instance Kang et al. [75] introduce low-rank approximation based computation and achieve better complexity.

2. Graph kernels based on subgraphs. The graphlet [100] and Neighborhood Subgraph Pairwise Distance kernel (NSPDK) [79] are popular instances of this family. Graphlet kernel counts the common non-isomorphic subgraphs (upto a certain degree) among a pair of graphs to evaluate their similarity and is computable in polynomial time. NSPDK counts the number of rooted subgraphs containing nodes up to a certain distance from the root, the roots of which are located at a certain distance from each other, in two graphs.

3. Graph kernels based on subtree patterns. WLK is the most popular instance of this family. Since we have explained it extensively in several sections, we refrain from discussing it here.

4. Deep Graph Kernels. Recently, the success of deep representation learning techniques in other fields like Natural Language Processing inspire researchers to learn representations of sub-structures from graph and thus develop deep learning variants of popular graph kernels. For instance, Yanardag and Vishwanathan [85] develop deep learning vari- 
ants of many of the above-mentioned kernels such as graphlet, SP and WLK.

Considering the malware detection application, while all these general purpose kernels could capture and compare structural data well, they fail to capture reachability contexts as it is a strong application-specific requirement. CWLK is specifically designed to address this research gap.

\subsubsection{Application-specific Graph Kernels}

Like CWLK, several application-specific kernels have been proposed in the past. For instance, Harchaoui and Bach [84] propose a segmentation kernel that counts common virtual sub-structures amongst images to perform Computer Vision tasks such as image classification. Mahe et al. [82] propose a marginalized graph kernel for a chemoinformatics application where they enrich the node labels using the Morgan index and counted the number of matching nodes across graphs. Driven by similar motivation, we have developed CWLK to cater specifically to malware detection. To the best of our knowledge, this is the first graph kernel specifically addressing a problem from the field of program analysis.

\subsection{Limitations}

The goal of CWLK is to capture both contextual and structural information from PRGs and it is a straight-forward extension of WLK. Though it is successful in this goal, it is plagued by limitations that are inherent due its WLK-like design. Both these kernels suffer with the limitations introduced in $\S 1.1$. In particular, they match PRG subgraphs based on syntactic similarity and this leads to: (i) diagonal dominance (see challenge C4.2 in 1.1) and (ii) inability to compute semantic similarities across PRGs (see challenge C4.1 in 1.1). These limitations are addressed through a representation learning approach in chapter 6, later. 


\subsection{Conclusion}

In this chapter, we present CWLK, a novel graph kernel that facilitates detecting malware using PRGs. Unlike the existing kernels which capture only the security-sensitive neighborhoods in PRGs, CWLK captures these neighborhoods along with the context under which they are reachable. This makes CWLK more expressive and in turn more accurate than existing kernels. Besides expressiveness, CWLK has two specific advantages: (1) shows high efficiency, (2) supports building explicit feature vector representations of PRGs. CWLK is evaluated on a large-scale experiment with more than 50,000 Android apps, and is found to outperform two state-of-the-art graph kernels and three malware detection techniques in terms of F-measure, while maintaining comparable efficiency.

Implementation \& Dataset. We provide an efficient implementation of CWLK and information on the datasets used within this work at: https://sites.google.com/site/ cwlkernel. 


\section{Chapter 4}

\section{Context-aware, Adaptive and Scalable}

\section{Android Malware Detection through}

\section{Online Learning}

In this chapter, CASANDRA framework that is capable of handling malware population drift, facilitating a scalable and explainable malware detection is presented. Some contents of this chapter are presented in a paper titled 'Adaptive and Scalable Android Malware Detection through Online Learning' in IJCNN 2016. Another paper of ours titled 'Contextaware, Adaptive and Scalable Android Malware Detection' which is published in IEEE Trans. of Emerging Topics in Computational Intelligence uses a portion of this chapter.

\subsection{Introduction}

The number of new Android malware apps have grown tremendously in recent years. For instance, Symantec reports [2] discovering 430 million new malware in 2015 which is a $36 \%$ increase over 2014. Also their capabilities have grown from simple phone cloning, sending premium-rated SMS to complex botnets, cryptolocker and ransomware [23, 4850]. Besides this, attackers continuously enhance the sophistication of malware to evade novel detection techniques. The sheer volume, growth rate and evolution of sophisticated 
Android malware highlights an imperative need for developing sound and scalable automated malware detection techniques $[23,25,26,48,50]$.

Machine Learning based malware detection. For over a decade, Machine Learning (ML) techniques have been predominantly used to perform malware detection in various platforms (such as Windows and Android) [23, 25, 26, 28, 29, 31, 32, 38, 48-50, 58, 113]. This is because, ML methods automatically learn the characteristics that distinguish malicious behavior, when trained using a collection of malware and benign samples making them amenable for automated detection. ML based approaches extract semantic features from apps' behaviors and apply standard classification algorithms (e.g.,SVMs, RFs, etc.) to perform binary classification. These approaches typically use features such as system calls/APIs invoked, resources and privileges used, control- and data-flows inside apps' execution to detect malicious behavior patterns [26, 29, 31, 32, 38, 58]. These semantic features are extracted through static $[25,26,28,29,32,58]$ and dynamic $[48,60]$ program analysis techniques.

\subsubsection{Malware Detection using Graph Representations}

Malware variants. As mentioned in the previous chapter, a major reason for the tremendous growth rate in malware is the production of malware variants. These variants perform same malicious functionality, with apparently different syntax, thus evading syntax-based detectors. However, PRGs such as CGs, CFGs and ICFGs which are higher level abstractions of the program functionalities tend to stay similar even when the code is considerably altered [26, 28, 29, 31, 32]. As PRGs are resilient against variants, many works in the past have used them for malware detection, essentially, casting the problem as a graph classification task.

Graph Mining and Kernels. Many existing works such as [35] and [36] use off-theshelf graph mining algorithms on PRGs for malware detection. However, as mentioned in 
the previous chapter, these graph mining based approaches have severe scalability issues, making them impractical for real-world malware detection.

On the other hand, one of the increasingly popular approaches in ML for graph-structured data is the use of graph kernels. These graph kernels could be used together with a kernel classifier (e.g., SVM) to perform graph classification [77]. Recently, efficient and expressive graph kernels such as [77], [78] and [79] have been proposed and widely adopted in many application domains (e.g, computer vision [84], chemoinformatics [82], etc.). These kernels have been known to operate in linear-time and have produced accurate results in many real-world applications. Therefore, it just suffices to apply any of these kernels on suitable PRGs and we have an effective, scalable and ready-to-use malware detector. However, as we explained in the previous chapter, these general purpose graph kernels do not take many problem-specific constraints and hence yield sub-par accuracies.

\subsubsection{Challenges in ML based Malware Detection}

Almost all ML based Android malware detection techniques (incl. the aforementioned ones) operate in a batch-learning setting and use off-the-shelf batch learners like SVM or RFs. Meaning, the detection model is built using a batch of labeled benign and malware samples and is subsequently used to predict whether a given new sample is benign or malicious.

In general, these batch ML based approaches are typically plagued by four challenges that make them unsuitable for large-scale real-world malware detection:

(C2.1) Population drift. Though batch-learning based solutions are promising, their success is predicated on an important assumption that may not hold for the malware detection problem. Meaning, they assume that the malware population (i.e., training data) used to build the detection model does not change over time. However, malware does not fit this profile. The entire population of malware is constantly evolving due to various reasons such 
as exploiting new vulnerabilities and evading novel detection techniques. This evolution has a profound impact on malware characteristics and thereby on the features used by these ML models. This makes the collection of malware identified today unrepresentative of the ones generated in the future. This phenomenon is an epitome of population drift $[50,113]$.

(C2.2) Explainability. In general, these ML based solutions just predict the labels of a given sample without offering insights or explanations into how those predictions are arrived at. In other words, they act as black-box solutions. However, for malware detection models, understanding the reasons behind their predictions is important in assessing their trustworthiness. This is fundamental if one plans to take action such as deploying a new model or studying malware evolution based on these predictions.

Additionally, in the case of PRG based approaches, as discussed in the previous chapter, retaining PRGs' expressiveness to achieve higher accuracies and performing scalable detection remain as known challenges. These challenges are briefly revisited in the wild malware detection context.

Scalability. Since the malware population grows at an alarming rate, a scalable classifier is of paramount importance for real-world malware detection. In order to keep abreast with drifting population, batch learners have to be frequently retrained with huge volumes of data. Hence they pose severe scalability issues when used in the Android malware detection context where we have thousands of apps streaming in every day. Retraining frequently with such a volume renders them computationally impractical.

Expressiveness. Only the approaches that leverage on PRGs face this challenge. In many cases vectorial representations of PRGs fail to capture all the vital information, thus losing their expressiveness. For instance, solutions like [31], [32] and [34] capture the topological neighborhood (i.e., structural) information from PRGs and detect security-sensitive behaviors through analyzing them. However, as revealed by recent works such as [29] and [28], an important contextual factor that distinguishes malice is whether or not the user 
is aware of such behaviors. Unfortunately, the above-mentioned methods which capture structural information well, fail to capture the contextual information and this leads to raising false alarms even when sensitive operations are performed with users' consent. The general purpose graph kernels such as $[74-79,100]$ also suffer with the same drawback.

\subsubsection{Our Approach}

We take these four challenges into consideration and propose CASANDRA (C्Context-aware,

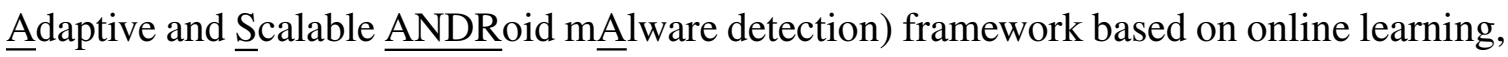
where we continuously retrain the model upon receiving each labeled sample and make prediction of a new sample using the updated model. CASANDRA is developed with the four following design goals:

1. Accuracy. Accuracy of CAsandra, which is a PRG based approach depends on how well it retains its expressiveness. To this end, we leverage on our work from the previous chapter (see chapter 3) and use CWLK that is specifically designed to capture both structural and contextual information from PRGs.

2. Efficiency. Casandra achieves its efficiency through the combined use of a scalable graph kernel (i.e., CWLK) and a state-of-the-art online classifier, namely, CW learner [117].

3. Adaptiveness. CASANDra automatically adapts to malware population drift through its use of online classifier and thus addressing challenge $\mathrm{C} 2.1$.

4. Explainability. Since CASANDRA uses a linear classifier along with CWLK which permits explicit feature vector representation of PRGs, we are able to categorically identify the PRG subgraph features which contribute to its predictions. This makes Casandra's predictions explainable thus addressing challenge C2.2.

Experiments. CASANDRa is evaluated through large-scale experiments both on benchmark and a recent wild dataset of more than 87,000 apps. CASANDRA achieves $89.92 \%$ accu- 
racy on the wild dataset, outperforming two state-of-the-art techniques by more than $25 \%$ in their typical batch-learning setting and more than $7 \%$ when they are continuously retrained, while maintaining comparable efficiency. Through further experiments, we demonstrate CASANDRA's explainable detection process and its adaptiveness.

Contributions. We make the following contributions in this chapter:

- We propose and develop CASANDRA, a context-aware, adaptive, scalable and explainable Android malware detection framework which is based on online learning, where we do not assume that the malware population is stationary.

- Adaptiveness is a unique feature of CaSANDra. We thoroughly demonstrate how CASANDRA adapts to malware population drift through carefully designed experiments with real-world malware. To the best of our knowledge, none of the existing approaches possess this capability.

- Performing explainable malware detection is a salient feature of Casandra. As we demonstrate through our experiments, CASANDRA's explanations are more comprehensive and semantically closer to malicious behaviors than those of state-of-the-art approaches.

Organization. The remainder of the chapter is organized as follows: we begin by introducing the background and motivations for our framework’s design in $\S 4.2$. The proposed CASANDRA framework is presented in $\S 4.3$. The experimental design and implementation details are furnished in \$4.4. CASANDRA's evaluation results and discussions are presented in section $\S 4.5$. Related Work, Limitations and Conclusions are provided in $\S 4.6, \S 4.7$ and $\S 4.8$, respectively. 


\subsection{Motivations}

In this section, we present our motivations and explain why using batch learning is impractical for building a real-world malware detector and how the use of online learning alleviates such practicality issues. Remember, the motivations for capturing contextual information along with structural information from PRGs is explained in the previous chapter.

As foreshadowed in $\S 4.1$, the three main motivations for using online learning instead of batch learning in CASANDRA are: (1) handling population drift, (2) handling large volumes of high dimensional data, and (3) performing detection on data that streams-in at realtime. Malware detection-specific justifications on how online learning helps to address these challenges are presented in separate sub-sections.

\subsubsection{Handling Population Drift}

As reported in [24], the attack and the evasion strategies of malware have evolved significantly, ever since the inception release of Android. Also, one could observe the following pattern in this evolution: attacks that were popular at some point in time could not sustain forever. For instance, the BaseBridge family of malware leveraged on two root exploits, namely, RATC and Zimperlich to escalate its privileges [24]. Once the corresponding vulnerabilities were patched and the AV vendors became capable enough to detect such attacks, BaseBridge's propagation and sustenance were affected, ultimately resulting in its extinction. This leads to the following observation: several malware families emerge, flourish and fade-away over time due to various domain-specific reasons.

From an ML based malware detection viewpoint, this leads to emergence, dominance and disappearance of semantic features that characterize these attacks and evasion strategies. This results in the three following phenomena that happen over time: (1) new features emerge (2) the significance of features vary (3) the cumulative number of features keeps 
monotonically increasing. This causes the underlying distribution of malware samples to change over time leading to what is known as a concept drift. As a result of this drift, the predictions of a static model might become less accurate in due course of time. Therefore, detectors need to adapt to such changes quickly and accurately.

A straightforward solution to this problem is to detect the magnitude and direction of drift in the concept over time and retrain the batch models when the drift is significant [50]. However, this approach would be hampered if: (1) the data arrives in large volumes at a rate too fast to detect, or (2) retraining the models at regular intervals is too expensive. Unfortunately, both these conditions are true in the real-world malware detection setting, as we review in detail below. On the other hand, online learners which are trained on a stream of samples as they arrive are naturally adaptive to evolving distributions mainly due to their learning mechanisms that continuously and efficiently update the model with the most recent examples.

\subsubsection{Handling Large High-dimensional Data}

Particularly in the malware detection problem, data is large not only in sample size, but also in feature size. For instance, on the large-scale dataset used in our evaluation, even a light-weight method such as DreBIN [25] extracts and uses more than 1 million semantic features (see §4.5.1). In such big data contexts, the efficiency of a model in terms of both memory and time is of paramount importance in determining its practicality.

However, many batch learners (e.g., SVMs, RFs) typically take multiple passes over the training samples to optimize their parameters and yield the most accurate models within their hypothesis space. Often times, they are optimized to have long and computationally expensive training phases in order to perform accurate and faster during testing. Also, they might require large memory as they hold a batch of samples in memory facilitating them to take multiple passes. On the other hand, online learners take exactly one pass over training 
data. Meaning, they look at each sample that streams-in only once and learn from them, without storing or iterating over them. This makes the online learners highly efficient in terms of both memory and time. Therefore, online methods offer more practically tractable solutions for malware detection over their batch counterparts.

\subsubsection{Handling Real-time Streaming}

Android has a vibrant and growing app ecosystem with a considerable number of thirdparty markets. Google Play [4], the official market, host more than 2.4 million apps as of this writing. Meaning, nearly 1120 new apps are submitted to Google Play, on average every day. Also, popular third-party markets (e.g., [5]) receive a similar number of new submissions on a daily basis.

Clearly, one important use-case of malware detection solutions like CASANDRA is to deploy them to perform automated vetting of apps submitted to these markets. Therefore, such solutions must be scalable, always up-to-date in terms of their knowledge on attack and evasion strategies and ready for predicting and offering insights into apps as and when they stream in. In other words, these model could not be trained with an outdated set of samples and could not take outages or downtime to retrain themselves. Unlike online methods, the batch learners are susceptible to both these disadvantages, making them unsuitable for handling classification over a continuous, fast and voluminous stream of apps.

\subsection{Methodology}

The methodology of CASANDRA framework designed to perform context-aware, scalable, adaptive and explainable malware detection is presented in this section. We begin by presenting the framework overview and subsequently, we explain each component of the framework. 


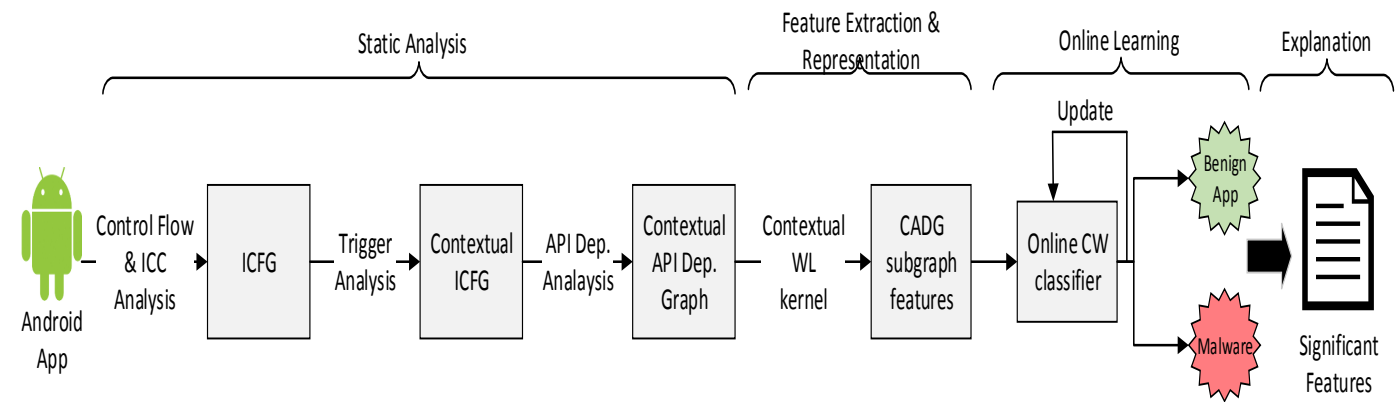

Figure 4.1: CASANDRA: Framework overview

\subsubsection{Casandra: Framework overview}

Fig. 4.1 depicts CASANDRa framework's overview. The framework has four components as described below.

1. Static Analysis. Our framework considers subgraphs from Contextual API Dependency Graphs (CADGs) as semantic features to perform malware detection. To this end, we first perform static program analysis to transform the given set of apps into their corresponding CADG representations. This procedure is explained in detail in section $§ 4.3 .2$.

2. Feature Extraction \& Representation. Once the CAGD of the apps in the dataset are constructed, those subgraphs which represent the security-sensitive events that happen in every app along with their context are extracted as using CWLK. We follow a BoF model [77] to construct the feature vector of individual apps. The detailed procedure is explained in $\S 4.3 .3$.

3. Online Learning. Once the feature vectors of all the apps in the training set are built, we train a CW classifier with them to detect malware. CW classifier's training and update procedures are explained in detail in $\S 4.3 .4$. Subsequent to this training CASANDRA is ready to perform malware detection at scale. It is important to note that the classifier is trained in an online fashion, meaning whenever a sample is presented, the classifier not only predicts its label but also updates the model based on the actual label of the sample.

4. Explanation. ML based malware detection solutions are usually black-box methods as 
they do not explain why a particular sample is detected as malicious or benign. In CASANDRA, we address this shortcoming as follows: besides detection CASANDRA reports significant CADG subgraphs of an app that contribute to a detection. In most cases, these significant subgraphs reveal the app's characteristics related to malicious or benign behaviors. The detailed procedure is presented in $\S 4.3 .5$.

\subsubsection{Static Analysis}

CASANDRA considers CADG subgraphs as features which encompasses both structural and contextual information characterizing security sensitive operations from apps. We perform a comprehensive static analysis on a given app to construct its CADG. Our static analysis and CADG construction process is very similar to that of DroidSIFT [29] and AppContext [28].

The CADG construction workflow as depicted in Fig. 4.1 involves three steps. Each of them are described below.

Step 1: ICFG Construction. The procedure mentioned in [28] is followed to construct the ICFG of a given app. Intuitively, the nodes of ICFG are the instructions in every method of the app. The ICFG edges are the intra- and inter-procedural control flows among these instructions. There two types of interprocedural invocations in Android apps: (1) method invocations and (2) Inter Component Communications (ICCs). Method invocations could be directly inferred through control flow analysis. However, ICCs are not straight forward as these invocations are facilitated by the underlying Android OS framework. In order to model such invocations, we leverage on the IC $3^{1}$ tool [57] and perform a precise ICC analysis.

Step 2: Contextual ICFG (CICFG) Construction. During the course of execution of the program, the ICFG nodes could be reached by actions triggered by entities such as ${ }^{1}$ It is noted that both DroidSIFT and AppContext used Epicc [56] for their ICC analysis. We have replaced it with IC3 which is more recent and state-of-the-art. 
user or system. Trigger events are the external events such as users' interaction with app's user-interface (UI) and system changes such as receipt of SMS that trigger invocation of security-sensitive APIs. Trigger events connect security-sensitive behaviors to their "initiator" in the external environment (e.g., users or system). DroidSIFT [29] proposed a method to analyze the entry point nodes (i.e., PRG nodes that do not have any incoming edge) to identify and categorize the trigger events into UI and NON-UI triggers. We extend this procedure and categorize trigger events of ICFG nodes into the three following types:

1. UI triggers: events triggered by interactions on apps' UI (e.g, Clicking UI buttons to make calls, etc.).

2. NON-UI triggers: events initiated by the system state changes (e.g., receipt of SMS, change of signal strength), and events initiated by hardware related actions (e.g., pressing the HOME or BACK button).

3. UNRESOLVED triggers: Entry points of certain sensitive methods are not traceable by our static analysis. For instance, DroidKungFu, a popular malware family has its malicious payload triggered by dynamically loaded code. However, similar to [29] and [28], our static analysis cannot model dynamic code loading and reflection based triggers. Therefore, we consider them as behaviors with UNRESOLVED trigger events rather than ignoring them.

Understandably, each ICFG node is triggered by one or more of these triggers. We consider ICFG nodes that are reachable through UI and NON-UI triggers to be in the user-aware and user-unaware contexts, respectively. Nodes that are reached through UNRESOLVED triggers are considered to be in the 'unresolved' context. The reachability context(s) of every node is added as a node attribute. This transforms ICFG to CICFG. To formally define CICFG, we adopt and extend the definition of an ICFG from Harrold et al. [89] as follows. 
Definition 4.1 (CICFG). $C I C F G=\left(N_{i}, E_{i}, \xi\right)$ of an app $a$ is a directed graph in which each node $n \in N_{i}$ denotes an instruction in every method of $a$, and each edge $e\left(n_{1}, n_{2}\right) \in$ $E_{i}$ denotes either an intra-procedural control-flow dependence from $n_{1}$ to $n_{2}$ or a calling relationship from $n_{1}$ to $n_{2}$. $\xi$ is a set of contexts though which every node $n \in N$ could be reached.

Step 3: CADG construction. The CICFG captures the complete control-flow across every instruction in an app, along with context information. However, only a selected minority of these CICFG nodes will be security-sensitive (i.e., will invoke sensitive APIs). Therefore, once the CICFG of an app is constructed, we abstract it to obtain CADG and narrow down our focus only to its sensitive behaviors. Intuitively, CADG of an app is obtained from its CICFG by considering only the nodes that access security-sensitive APIs ${ }^{2}$ along with their context information. All other nodes are removed and paths that exists between such nodes in the CICFG become edges in ADG, if they satisfy certain conditions as described below.

Definition 4.2 (CADG). CADG can be represented as a 4-tuple $C A D G=(N, E, \lambda, \xi)$, where $N$ is a finite set of nodes and $n \in N$ is an instruction of a method that corresponds to invoking a security-sensitive API and therefore $N \subseteq N_{i} . E \subseteq N \times N$ is a set of directed edges where an edge from $e\left(n_{1}, n_{2}\right) \in E$ exists iff there exists a path $p\left(n_{1}, n_{2}\right)$ between these two nodes in the CICFG and $\operatorname{method}\left(n_{1}\right)=\operatorname{method}\left(n_{2}\right)$, where $\operatorname{method}(n)$ denotes the method that encompasses instruction $n^{3} . \lambda$ is the set of labels representing the security-sensitive APIs and $\ell: N \rightarrow \lambda$ is a labeling function which assigns a label to each node. $\xi$ is a set of ${ }^{2}$ Two existing works, namely, PScout [51] and SUSI [52] list commonly known security-sensitive An-
droid APIs. These two lists have been used to perform security and malware analysis by many existing
approaches such as [28,54,58]. Following these studies, CASANDRA uses these two lists to identify security-
sensitive APIs.
3This follows from the observation that in most malware the malicious code portion is closely-knit i.e.,
spanning only to a few methods. We also attempted two other variants of CADG. We reduce the path in
CICFG to edges in CADG (i) only if the calling and called nodes belong to the same package and (ii)
only if the calling and called nodes belong to the same class. Both these variants contained much larger
number of edges in CAGD and also failed to capture the attacks as effectively as the CADG defined above
(experimentally verified). 
triggers though which every node in the CADG could be reached and $C \rightarrow \xi$ is a function which assigns the context to each node.

To provide example illustrations of CADGs, we reuse some content from the previous chapter. A portion of Geinimi and Yahoo Weather examples' CADGs are shown in Fig. 3.1 (c) and (d), respectively (in this case of this code snippet, the Contextual Data Dependency Graph is same as its CADG). In both these apps, all three CICFG nodes invoke securitysensitive APIs and they are retained in CADGs. All of these nodes belong to the same method (i.e. leak_info_to_url) and hence the path that existed among them in CICFG translate to edges in CADG.

\subsubsection{Feature Extraction \& Representation using CWLK}

Once the CADGs are constructed, contextual subgraphs that represent the security-sensitive behaviors in every app are extracted from them using CWLK. Subsequently, CADGs are represented as feature vectors.

As discussed in detail in the previous chapter (chapter 3), given a PRG, $G=\{N, E, \lambda, \xi\}$, CWLK works by augmenting the labels of every node $n \in N$ with its neighborhood and the context through which it could be reached. The frequency of these contextual subgraphs around every node are used as features to facilitate an explicit feature vector representation of $G$.

Thus the process of obtaining feature vector representation of CADGs in our dataset using CWLK involves two key steps: (1) Building a vocabulary, $\mathbb{V}$ of contextual sub-graph features present across all CADGs, (2) Transforming every CADG into a feature vector with $|\mathbb{V}|$ dimensions. This process is presented in detail in Algorithm 2.

The inputs to the algorithm are $\mathbb{G}$, set of all CADGs and $h$, the height of CWLK (i.e., number of times contextual relabeling has to be performed on every node). The output is the set of feature vectors of all the CADGs, $\mathbb{X}$. 


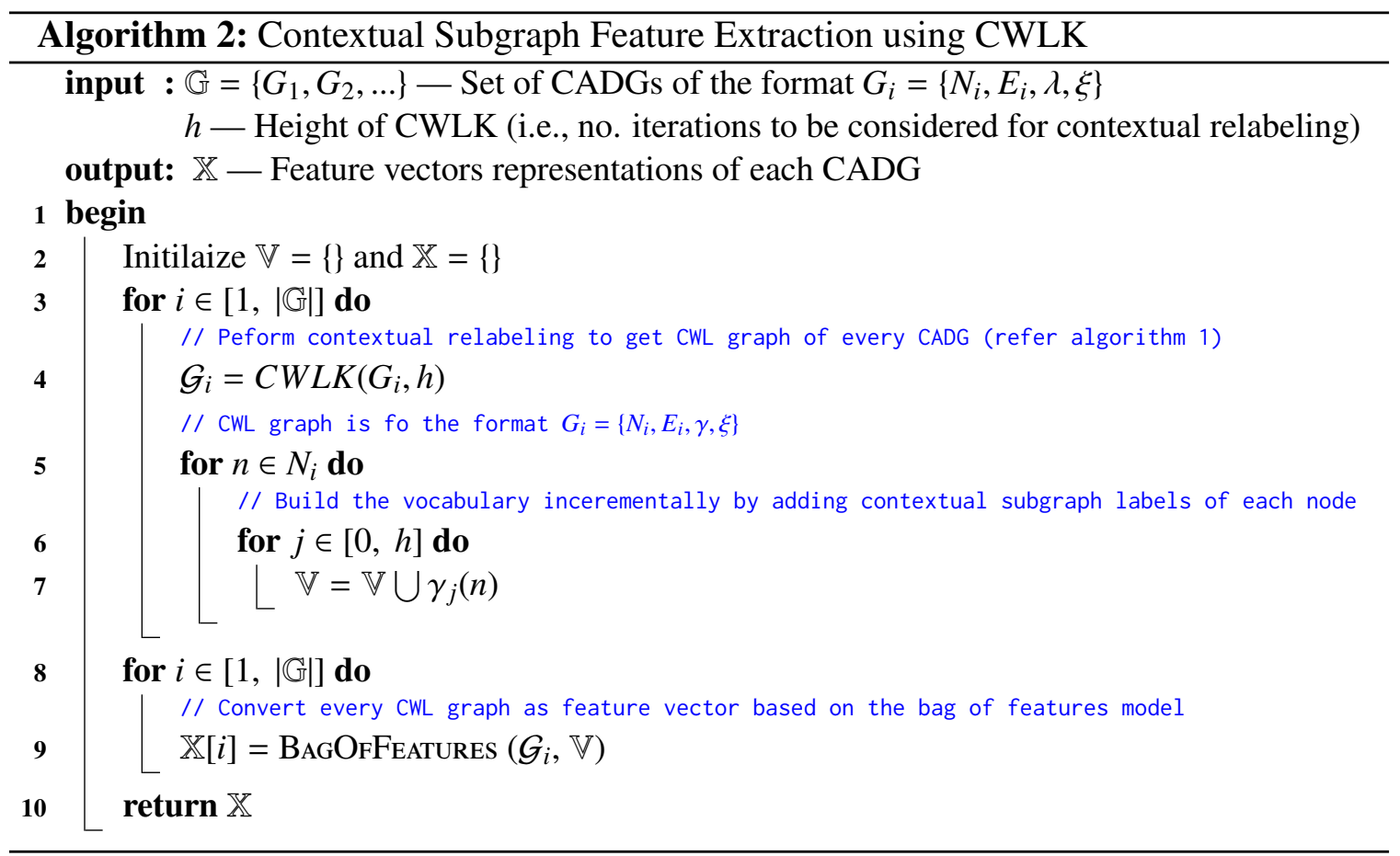

To begin with we initialize vocabulary of contextual subgraphs, $\mathbb{V}$ and the set of feature vectors, $\mathbb{X}$ to empty sets (line 2 ). Then for every CADG, $G_{i} \in \mathbb{G}$, we perform contextual relabeling using algorithm 1 (proposed in chapter 3) (line 4). This yields the CWL graph corresponding to $G_{i}$ which contains contextual subgraph labels $(\gamma)$ of every node $n \in N_{i}$. Then, for every node $n$, we extract all its contextual subgraph labels and add the same to the vocabulary $\mathbb{V}$ (lines 5 to 7 ). This helps to build the vocabulary incrementally with unique contextual subgraphs across all CADGs in $\mathbb{G}$.

Once the vocabulary $\mathbb{V}$ is obtained, we transform every $C A D G G$ into its corresponding vector representation by counting the frequency of every contextual subgraph feature from $\mathbb{V}$ (lines 8 and 9). This procedure falls under the well-known BoF representation model (discussed elaborately in 3.4.2), where every CADG is considered as a bag of contextual subgraphs. Finally, $\mathbb{X}$, the set of feature vectors of all CADGs are returned in line 10. 


\subsubsection{Online Learning}

Once the feature vectors of all the apps in the training-set are built, we train an online CW [117] classifier with them to detect malware. CW classifier's training and update procedures are as explained below with relevant notations.

Denote the features of an app (both benign and malware) as a vector $\overrightarrow{\mathbf{x}}=\left[x^{(1)}, x^{(2)}, \ldots, x^{(d)}\right]^{T}$, and its label as $\mathbf{y} \in\{-1,+1\}$, where -1 indicates benign and +1 indicates malicious apps. The CW classifier receives a number of samples, $\mathbf{x}_{i}$, and their labels, $\mathbf{y}_{i}$, and trains using this labeled data. Given a new unseen sample, $\mathbf{x}$, the goal of $\mathrm{CW}$ classifier is to predict the label, $\mathbf{y}$, of this new sample based on its trained model.

CW classifier fits a linear decision boundary (i.e., hyperplane) between the positive and negative class samples. That is, the model is a weight vector, $\overrightarrow{\mathbf{w}}=\left[w^{(1)}, w^{(2)}, \ldots, w^{(d)}\right]^{T}$ which indicates the weight (i.e., relative importance) of each of the features used to predict the output label $\mathbf{y}$. The predicted label, $\hat{\mathbf{y}}$, is the sign of the inner product between $\overrightarrow{\mathbf{x}}$ and $\overrightarrow{\mathbf{w}}$ :

$$
\hat{\mathbf{y}}=\operatorname{sign}(\overrightarrow{\mathbf{x}} \cdot \overrightarrow{\mathbf{w}})
$$

$\mathrm{CW}$ incrementally builds the model in eq. (4.1) in rounds. In round $t$, it receives a sample, $\mathbf{x}_{t}$ and predicts its label $\hat{\mathbf{y}}_{t}$ using the current model; it then receives $\mathbf{y}_{t}$, the true label of $\mathbf{x}_{t}$ and updates its model based on the sample-label pair: $\left(\mathbf{x}_{t}, \mathbf{y}_{t}\right)$. A Gaussian distribution over the weights with mean $\vec{\mu}$ and covariance matrix $\Sigma$ is maintained by the $\mathrm{CW}$ algorithm. The value $\mu^{(\mathfrak{f})}$ represents mean weight of feature $\mathfrak{f}$ (i.e., mean of $\left.w^{(\mathfrak{f})}\right)$, and the value $\Sigma_{\mathfrak{f}, \mathfrak{f}}$ captures the confidence in $\mathfrak{f}$ 's weight. While classifying a new sample $\mathbf{x}$, the weight $\overrightarrow{\mathbf{w}}$ is drawn from $N(\mu, \Sigma)$. In practice, one could choose $\overrightarrow{\mathbf{w}}=\vec{\mu}$, the average weight vector and use eq (4.1) to arrive at the predicted label. CW updates the model, (i.e., $\vec{\mu}$ and $\Sigma$ ), continuously on every labeled sample instead of only when committing misclassifications. The rationale is that making correct prediction also suggests that the model should increase 
its confidence of the current weights. CW's update rule is presented below:

$$
\begin{gathered}
\left(\vec{\mu}_{t+1}, \Sigma_{t+1}\right)=\arg \min _{\vec{\mu}, \Sigma} D_{K L}\left(\mathcal{N}(\vec{\mu}, \Sigma) \| \mathcal{N}\left(\vec{\mu}_{t}, \Sigma_{t}\right)\right), \\
\text { s.t. } \operatorname{Pr}_{\overrightarrow{\mathbf{w}} \sim N(\vec{\mu}, \Sigma)}\left[y_{t}\left(\overrightarrow{\mathbf{w}} \cdot \overrightarrow{\mathbf{x}}_{t}\right)\right] \geq \eta .
\end{gathered}
$$

Eq. (4.2) determines that the new distribution characterized by new $\Sigma$ and $\vec{\mu}$ should be close to the old distribution as much as possible. The KL divergence $\left(D_{K L}\right)$ provides the measure of distance between the two distributions. Eq. (4.3) determines that the update should ensure that the probability of making correct prediction if the same sample, $\mathbf{x}_{t}$ is seen in the next round must be bigger than $\eta$, where $\eta$ is a configurable parameter (usually set bigger than 50\%). The computational complexity of the update is linear in the number of non-zero features in $\mathbf{x}_{t}$.

The strength of CW lies in its notion of modeling confidence on features' weights. Evidently, if the weight of a feature does not fluctuate very much over time, one could confidently believe that this weight is what it should be. CW achieves the two following desirable characteristics through this confidence notion, which are not exhibited by other online learners (e.g., OP, PA):

1. The weights of more confident features are updated less aggressively. For instance, using sendSms API in the user-unaware context is a good indicator of an app's maliciousness; consequently, its weight does not get updated abruptly over time, thereby instigating high confidence on this feature. Therefore, $\mathrm{CW}$ ensures that the weight will not change much even when it receives an instance of benign app using user-unaware \{sendSms\}, which possibly could be a case of incorrect labeling. This makes CW naturally robust to labeling noise.

2. The model is updated just enough to adapt to the changing trends in the data while re- 
fraining from changing too much. The rationale is that the previous model carries valuable information about the data and should not be modified too abruptly.

Alternatives. As practitioners involved in building an online malware detection framework, we do not have any vested interest in a particular algorithm for online learning. Ultimately, we wish to determine the algorithm that scales well to problems of our volume and complexity and yields the best performance. To that end, we experimented with other well-known online learning algorithms such as OP [114] and Stochastic Gradient Descent learning based Logistic Regression ( $\mathrm{LR}_{\mathrm{SGD}}$ ) [115] and PA [116], along with CW. Since CW offered the best results in terms of accuracy and efficiency, it is used in CaSANDRA.

We believe this is because $\mathrm{OP}$ and $\mathrm{LR}_{\mathrm{SGD}}$ do not imbibe the notion of classification confidence and treat all misclassifications equally. PA, on the other hand, updates more aggressively when the margin of error is large and less aggressively, otherwise. Meaning, even though PA imbibes confidence of the classification decision, it does not account for the confidence over individual features. This leads PA to vary the weights of features rapidly and thereby making it susceptible to fitting to noise. On the contrary, $\mathrm{CW}$ imbibes both the aforementioned confidences and thus is robust to noise in the stream.

Online Kernel Classifiers. We experimented with online kernel (i.e., nonlinear) classifiers such as Projectron [119] and Forgetron [118] (with budget). Since these algorithms offered marginal improvement in accuracy at a significantly worse efficiency in our largescale dataset, we chose $\mathrm{CW}$ to cater CASANDRA's online learning requirements.

Overall, as the CW algorithm makes a fine-grained distinction between each feature's weight confidence, it can be especially well-suited to detect malware apps since our data stream continually introduces a dynamic mix of new and recurring CADG subgraph features. Once the $\mathrm{CW}$ classifier in CASANDRA is trained with all the samples it is ready to perform malware detection at scale. 


\subsubsection{Explaining Casandra's predictions}

Once CASANDra classifies a sample as malicious or benign, the next step is to offer explanations of these predictions by reporting significant CADG subgraphs of an app that contribute to prediction. These explanations help to understand whether the rationale behind the model's predictions are reasonable, thus ensuring users' trust on the model. Besides, they could help human analysts in several tasks such as studying malware attacks and creating malware signatures.

Explaining the predictions of linear classifiers is a well-studied problem [25, 32, 88]. Following them, we are able to determine the contribution of each CADG subgraphs feature to the final class prediction as described below.

Based on eq. (4.1) in section $\$ 4.3 .4$, for a given sample $\mathbf{x}$, during the prediction of $\hat{\mathbf{y}}$, the largest $v$ weights $w^{(s)}$ which are significantly important for placing the sample on the malicious (or benign) side of the decision boundary are identified (i.e., a point-wise multiplication $\overrightarrow{\mathbf{w}} \times \overrightarrow{\mathbf{x}}$ is performed and $v$ largest values are obtained from the resulting vector $w x$ ). Since each weight $w^{(s)}$ is assigned to a certain feature $x^{(s)}$, it is then possible to explain why an app has been classified as malicious (or benign). After extracting the top $v$ CADG subgraph features, CASANDRA reports them explaining the significant semantic characteristics that make a particular sample malicious (or benign). As we could observe from our evaluations, the frequently observed malware features include CADG subgraphs representing the use of reflection, dynamic and native code, accessing private information, sending of SMS and communication over internet predominantly in the user-unaware or unresolved context. 


\subsection{Experimental Design \& Implementation}

We conducted several large-scale experiments to evaluate CASANDRA's accuracy, efficiency, adaptiveness and explainability which are its primary design goals. We also compare its performance against two accurate and efficient state-of-the-art malware detection approaches. In this section, experimental design aspects such as RQs addressed, datasets used, evaluation setup and metrics are presented along with implementation details.

\subsubsection{Research Questions}

We intend to address the following RQs through our evaluations:

(RQ1 Accuracy) Does CASANDRA's online learning provide any benefit over batch learning in terms of malware detection accuracy?

(RQ2 Efficiency) How efficient is CASANDRA over batch learning based solutions in terms of overall training and prediction time?

(RQ3 Explainability) How explainable are CASANDRA's predictions?

(RQ4 Adaptiveness) How adaptive is CASANDRA when performing malware detection in the real-world setting? In other words, does it adapt to malware population drift seamlessly?

\subsubsection{Dataset}

CASANDRA's accuracy and efficiency are evaluated on a large dataset of wild apps. Its explainability and adaptiveness are evaluated on on well-known benchmark datasets (for reasons explained below). The composition of these datasets are as follows:

In-the-wild (ITW) dataset. This dataset comprises of 87,257 apps collected in-thewild. These apps are collected from seven different Android markets, namely, Google Play, Anzhi, AppChina, SlideMe, HiApk, FDroid and Angeeks, in 2014. Following the common 
Table 4.1: In-the-wild Dataset with apps dated from Jan 12014 to Aug 122014

\begin{tabular}{|l|c|c|}
\hline Market & \# of Benign Apps & \# of Malware \\
\hline Google Play [4] & 39156 & 26178 \\
\hline Anzhi [6] & 2957 & 12260 \\
\hline AppChina [7] & 1845 & 4154 \\
\hline SlideMe [8] & 289 & 132 \\
\hline HiApk [9] & 65 & 157 \\
\hline FDroid [10] & 29 & 2 \\
\hline Angeeks [11] & 6 & 27 \\
\hline
\end{tabular}

practice in software security research, we use the Virus Total web portal [15] which hosts malware detection services from more than 40 Anti-virus scanners to determine the groundtruth labels of the apps. We infer that dataset contained 44,347 benign and 42,910 malware apps. The composition of the dataset is presented in Table 4.1. It is noted that this is a subset of a large collection of apps used in [26].

The date of creation of these apps fall in a span of 224 days starting from 1 Jan'14 to 13 Aug'14. We intend to sort these apps according to their date of creation and emulate a live feed of apps to the malware detectors considered in our experiments to examine how they cope up with the drift in the malware characteristics over time. To this end, we divide all the apps in the dataset (both benign and malware) into batches according to their date of creation. Hence we have 224 batches, one for each day. The resulting time-line based distribution of the two datasets are presented in Fig. 4.2.

Benchmark dataset. In case of RQs 3 and 4, apart from the ground truth on whether or not an app is malicious, we need the name of the family to which it belongs. This helps to correlate an app's behavior to the common behaviors exhibited by other variants of its family. However, precise family labels for apps in the ITW dataset are not available. Hence in these RQs, we use the Drebin dataset introduced in chapter 3 (see §3.5). To distinguish it from the ITW dataset, it is referred as DR dataset. 


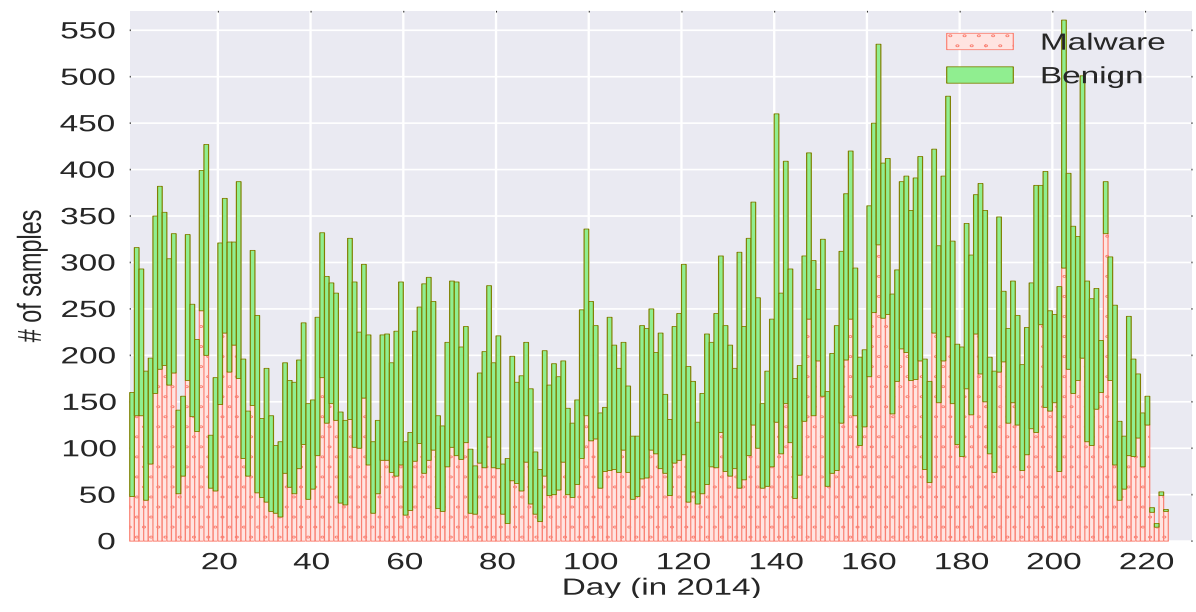

Figure 4.2: Timeline based distribution of apps in our large-scale ITW dataset (with malware and benign apps proportions).

\subsubsection{Experimental Setup}

All the experiments were conducted on a server with 36 cores of Intel(R) Xeon CPU E52699 v3 @ 2.30GHz and 32 GB RAM running Ubuntu 14.04.

\subsubsection{Implementation and Comparative Analysis}

CAsandra is implemented in approximately 17,200 lines of Python and Java code. IccTA [54] an Android static analysis workbench based on Soot [17] (which encompasses IC3 [57] for ICC analysis) is used for building CADGs from apps. Scikit-learn [87] toolbox is used for CASANDRA's ML functionalities.

Similar to the evaluations in chapter 3, CASANDRA is compared against two state-of-theart Android malware detection solutions, namely, DreBIN [25] and CFG Signature Based Detection (CSBD) [26].

\subsubsection{Evaluation Metrics}

Standard evaluation metrics such as Precision, Recall, F-measure and cumulative error rates are used to determine the effectiveness of malware detection. All these values are in the 
range $[0,1]$. High values of precision, recall and F-measure and low values of cumulative error rate indicate accurate detection. Efficiency is determined in terms of time required to train and test the classifiers. Lower values of training and testing durations indicate efficient detection.

\subsection{Evaluation}

The evaluations, results and discussions pertaining to each of the RQs are presented in this section.

\subsubsection{RQ1: Accuracy}

In this RQ, we now intend to evaluate the benefit of using online over batch learning for malware detection.

Dataset \& Experiment. In this experiment, we use the ITW dataset as it is collected in the wild and larger, thus offering more scope for illustrating malware population drift. We divide the ITW dataset apps (both benign and malware) into batches according to their date of creation. The resulting time-line based distribution of the two datasets are presented in Fig. 4.2. We have 224 batches, one for each day of the ITW collection.

Specifically, in this experiment, we intend to illustrate the benefits that CASANDRA attains due to its use of online learning. To study this impact, we replaced the online learner in CASANDRA with a canonical batch learner, namely, SVM and compare the same with CAsandra under different experimental settings (which involve periodic retraining). The above-mentioned SVM variants use the same features as CASANDRA and hence it is sufficient to compare online and batch learning paradigms. Furthermore, in order to compare CASANDRA to state-of-the-art batch learning solutions, we include DREBIN and CSBD (which use different set of features) in this comparative analysis. 


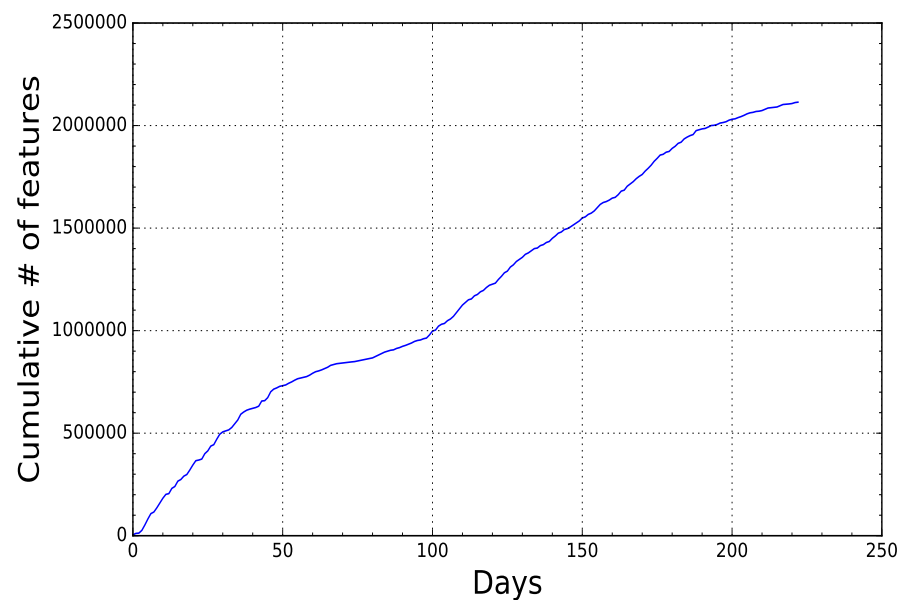

Figure 4.3: Cumulative number of features observed over time for our ITW dataset.

Batch Learning Configurations. For batch-learning solutions, the classifiers are trained/retrained in a sliding window fashion over the 224 batches as explained below. For SVM, we experiment with the following variants: SVM-Once, SVM-Daily, SVM-MultiOnce, and SVM-MultiDaily. For SVM-Once, SVM classifier is trained only once on the apps from Day 1 (1 Jan'14). This model is tested on all other days without retaining. For SVM-Daily, the classifier is retrained after every day; however, only one previous day's samples are used for every retraining - e.g., 11 Jan'14 results reflect training on the apps created on 10 Jan'14, and testing on 11 Jan'14 apps. SVM-MultiOnce is similar to SVM-Once and SVM-MultiDaily is similar to SVM-Daily, however, with the size of the batch for training and retraining covers 10 days instead of 1 . In summary, for Once and MultiOnce variants, the model is never retrained and for Daily and Multi-Daily, the model is retrained in a sliding window fashion over the batches of data. The size of MultiDaily training sets is determined to be 10-day batches based on the data that our evaluation machine can handle. Exactly same four variants were used for Drebin and CSBD.

CASANDra's online training regimen. Since CASANDRA's feature extraction using CWLK is based on BoF model, its number of features grows as the samples stream in. Fig. 4.3 shows the cumulative number of features for each day of the evaluation in ITW dataset, 
representing the feature space growth. Each day's total includes new features introduced that day and all the old features from previous days. We obtained a total of 15,171 features from the samples encountered on Day 1 (1 Jan'14). The dimensionality grows quickly as we extract new subgraph features from samples encountered every day and while reaching the final day (13 Aug'14), we have accumulated 2,114,050 features. This phenomenon of feature space growth is common across many techniques including DREBIN and CSBD. This is because apps evolve over time for various reasons such as capability enhancements, bug fixes and adapting to changes in Android framework APIs [25, 50, 68]. This evolution results in newly observed characteristics which translate into new features from an ML view-point.

Now, leveraging on the inferences from similar work [67], we refrain from using only a subset of these features (e.g., using only the 15,171 features encounter on Day 1). Instead, we allow the dimensionality of our CW classifier to grow with the number of new features encountered; on the last day (13 Aug'14), for instance, we classify with more than 2.1 million features. Implicitly, samples that were introduced before a feature $\mathfrak{f}$ was first encountered will have value 0 for feature $\mathfrak{f}$.

This training regimens helps our CW classifier stay abreast of changing trends in malware and benign apps' features.

Results \& Discussions. Figs. 4.4 (a), (b) and (c) shows the cumulative error rates of CASANDra in comparison to the aforementioned variants of SVM, DReBIN and CSBD. The following observations are made from the figure:

- Updating the detection models over time is essential to detect new malware as shown by SVM-MultiDaily and SVM-Daily outperforming SVM-MultiOnce and SVM-Once, respectively.

- Training on significantly more data improves the performance, as illustrated by SVMMultiDaily and SVM-MultiOnce outperforming SVM-Daily and SVM-Once, respec- 


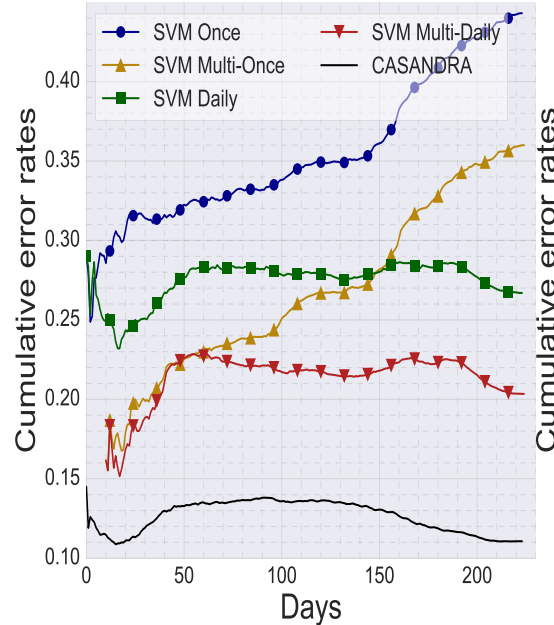

(a)

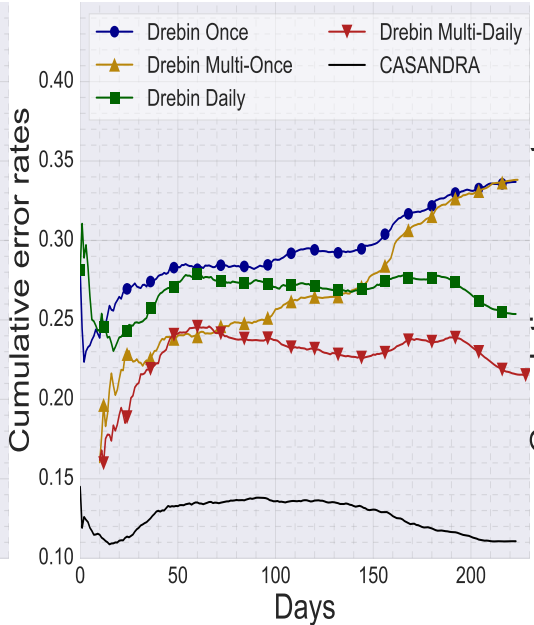

(b)

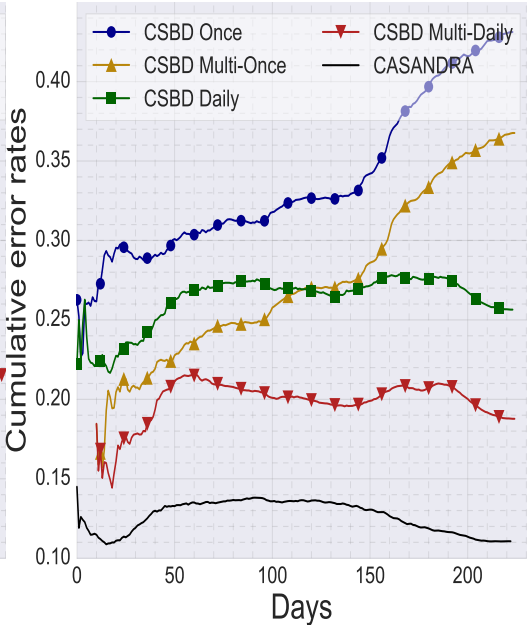

(c)

Figure 4.4: Casandra: : Cumulative error rates for CaSANDRa vs. batch learning algorithms (a) Casandra Vs. four variants of SVM, (b) Casandra Vs. four variants of Drebin, (c) Casandra Vs. four variants of CSBD.

tively. However, it is noted that there is a fundamental limit on the amount of data a batch-learning technique could train on because of the storage, memory and time requirements. Thus, among the SVM variants we have considered, SVM-MultiDaily achieves the best accuracy, as it has both the advantages of being trained frequently and trained with large volumes of data.

- From Fig. 4.4 (a), one could see that CASANDRA consistently outperforms all the batchlearning SVM variants. In particular, when the SVM models are not retrained, CASANDRA outperforms SVM-Once and SVM-MultiOnce by more than $32 \%$ and 25\%, respectively. When the SVM models are retrained on a daily basis, CASANDRA still outperforms SVMDaily and SVM-MultiDaily by more than $16 \%$ and $9 \%$, respectively. This is because CASANDRA is able to adapt to the changes in the malware characteristics instantaneously as well as retain significant useful information from the past.

- In the case of comparison with DREBIN and CSBD, the trends in the performance of all four variants of these methods are similar to those of the SVM variants. Hence the observations on frequently updating the models and training with more data, hold. 
- From Fig. 4.4 (b) and (c), one could see CASANDRA consistently outperforming the best performing variants of state-of-the-art methods. Particularly, it outperforms DrebinMultiDaily by $10.61 \%$ and CSBD-MultiDaily by $7.89 \%$. This reaffirms the suitability of online learning solutions over retrained batch learners for practical large-scale malware detection.

\subsubsection{RQ2: Efficiency}

In this RQ, we investigate the efficiency of CASANDRA in terms of training and testing durations. Specifically, we study whether CASANDRA achieves superior accuracy at the cost of very high (practically inviable) training or test durations.

Dataset \& Experiment. The ITW dataset which involves several thousands apps, instigating a vocabulary of over 2 million features for CASANDRA, 1 million features for DREBIN, offers enough opportunities to challenge these techniques in terms of efficiency. Hence we consider ITW dataset for this RQ. The experimental setting mentioned in RQ1 are reused in this RQ as well.

A typical batch learning test cycle will involve only feature extraction, representation and prediction on each test set sample. However, in the online learning setting the model will predict and at the same time learn from samples that stream-in. The initial online model is often built with a batch of trivial number of samples and keeps updating itself from the samples that stream-in. We emulate this scenario, as we use the batch of samples that stream-in on the first day of ITW dataset (i.e., 1 Jan'14) to build the model. The samples that arrive thereafter are considered as stream of samples on which CASANDRA predicts the label and also learns from.

Results \& Discussions. The training and the testing durations ${ }^{4}$ of CASANDRA and the 4

${ }^{4}$ These durations are different from training and testing durations of CWLK reported in the previous chapter (see 3.5.4, specifically, Fig. 3.4). As it is an embedding technique and not a classification technique, for CWLK, we included the feature extraction time in the training and testing duration. Here, in the case CASANDRA, as the focus is on classification speed, we just report the durations required to train and test the model after the feature vectors are obtained (i.e., feature extraction time is not included). 
Table 4.2: Comparing CASANDRA's efficiency against that state-of-the-art methods in terms of training and testing durations

\begin{tabular}{|l|l|l|}
\hline Method & $\begin{array}{l}\text { Training Duration } \\
\text { (avg. } \pm \text { std.) in sec. }\end{array}$ & $\begin{array}{l}\text { Testing Duration } \\
\text { (avg. } \pm \text { std. }) \text { in sec. }\end{array}$ \\
\hline DrebinOnce & $\mathbf{0 . 0 0 9 6}$ & $\mathbf{0 . 0 0 0 4}( \pm 0.00)$ \\
DrebinMultiOnce & 0.0352 & $0.0006( \pm 0.00)$ \\
DrebinDaily & $0.4493( \pm 0.08)$ & $0.0010( \pm 0.00)$ \\
DrebinMultiDaily & $0.5873( \pm 0.27)$ & $0.0010( \pm 0.00)$ \\
\hline CSBDOnce & 0.1849 & $0.0354( \pm 0.01)$ \\
CSBDMultiOnce & 0.5858 & $0.0594( \pm 0.03)$ \\
CSBDDaily & $11.0698( \pm 5.38)$ & $0.0605( \pm 0.02)$ \\
CSBDMultiDaily & $14.5157( \pm 11.40)$ & $0.0641( \pm 0.03)$ \\
\hline CASANDRA & 0.0131 & $0.0011( \pm 0.00)$ \\
\hline
\end{tabular}

variants of state-of-the-art solutions across all the 224 evaluation days in the ITW dataset are presented in Table 4.2. In the case of batch learning models, the training durations of variants that are retrained every day (i.e., Daily and MultiDaily) are distributions of values (one for each day). For the variants that are not retrained (i.e., Once and MultiOnce) the training durations are single values, as the training happens only once. In the case of testing durations, each model undergoes testing cycle every day and hence this produces a series of testing durations.

The following observations are made from Table 4.2:

- Understandably, the batch learning variants that are trained with smaller sets consume lesser training duration than their counterparts trained with larger sets. For instance, the training durations of Drebin-Once and CSBD-Once are more than 3 times shorter than those of their respective MultiOnce counterparts. Similar trend is observed when we compare the variants that are retrained. That is, on average, both Drebin-Daily and CSBD-Daily are trained 1.3 times faster than their respective MultiDaily counterparts. This shows that the training duration of the batch learning models increase exponentially with the training set size. For obvious reasons, the testing durations of Once and Daily variants are similar to those of their Multi counterparts for both techniques. 
- It is well-known the choice of the classifier plays a pivotal in determining the training and testing durations of the models. Linear models like Linear SVM and CW are simpler and could be trained much faster than quasi-linear models like RFs. This fact is evident from our findings in the aforementioned figure. All the variants of DREBIN and CASANDRA (which use linear models) are significantly faster than their CSBD counterparts (which use RF classifiers) in terms of both training and testing durations.

- Finally, we intend to compare CASANDRA's efficiency with those of best performing variants of the state-of-the-art methods (i.e., Drebin-MultiDaily and CSDB-MultiDaily). The training duration of CASANDra is 44 and 1108 times lesser than those of Drebin and CSBD MultiDaily variants, respectively. This clearly illustrates that retraining the models at specific intervals are impractical and much less efficient than online learning in the real-world setting. In terms of testing durations, CASANDRA is nearly 58 times faster than CSBD-MultiDaily and as fast as Drebin-MultiDaily. In summary, on the ITW dataset, CASANDra takes 28.23 microseconds on average to predict the class of a given sample.

\subsubsection{RQ3: Explainablity}

Apart from its detection performance the strength of CASANDRA lies in its ability to offer interpretations of the obtained results. We verify this quality of CASANDRA in this RQ.

Dataset \& Experiment. In this experiment, we intend to rank the features from a given malware sample that maximally influence CASANDRA's classification decision and investigate if they reflect the malicious behavior of the sample. If they do so, we can conclude that those features explain the sample's behavior the best. Understandably, the ground truth on the malware behavior could be ascertained by knowing the family to which it belongs. For instance, a sample belonging to the FakeInstaller family is expected to send premiumrated SMS as this behavior is a part of its attack vector. Therefore, clearly, the availability 
of malware family labels is indispensable for this experiment. Out of our two datasets, only the DR dataset contains malware family labels. Hence in this RQ, we experiment with three popular malware families from the DR dataset and analyze how CASANDRA features with high weights allow conclusions to be drawn about their behavior.

As in the previous RQs, we intend to compare the explainability of CASANDRA with that of DREBIN and CSBD. In terms of classifiers used, it is noted that results of DREBIN (which uses linear SVM) and CSBD (which uses RF) are perfectly interpretable. However, the features used by each of these methods are also different and have different levels of interpretability. As mentioned in the original work [25], a significant feature reported by DREBIN for the FakeInstaller family is the use of permission SEND_SMS. This is highly correlated to the main functionality of the malware. However, for the same family, CSBD, as it considers CFG signatures as features, reports the signature FOFOFOFOFOFOFOFOFOFOFOP1G as the most significant feature (see $\S 4.1$ of [26] for details on extracting CFG signature features). This feature is neither humanly interpretable nor correlated to FakeInstaller's malicious functionality. This clearly illustrates that CSBD's results are inexplicable. Hence, we refrain from including CSBD in this RQ's comparative analysis.

Results \& Discussion. To study the most significant features that influence the predictions of the CASANDRA and DREBIN, we consider three well-known malware families, namely FakeInstaller, GingerMaster and DroidKungFu from the DR dataset. For each sample of these families we determine the features with the highest contribution to the classification decision and average the results over all members of a family. The resulting top five features for each malware family for both the techniques are shown in Fig. 4.5.

From the figures the following inferences are drawn for each family:

FakeInstaller malware samples send premium-rated SMS to specific numbers without users' consent. It is evident that features identified by CASANDRA not only highlight neighborhoods with operations related to SMS, but also reflect that they are triggered pre- 


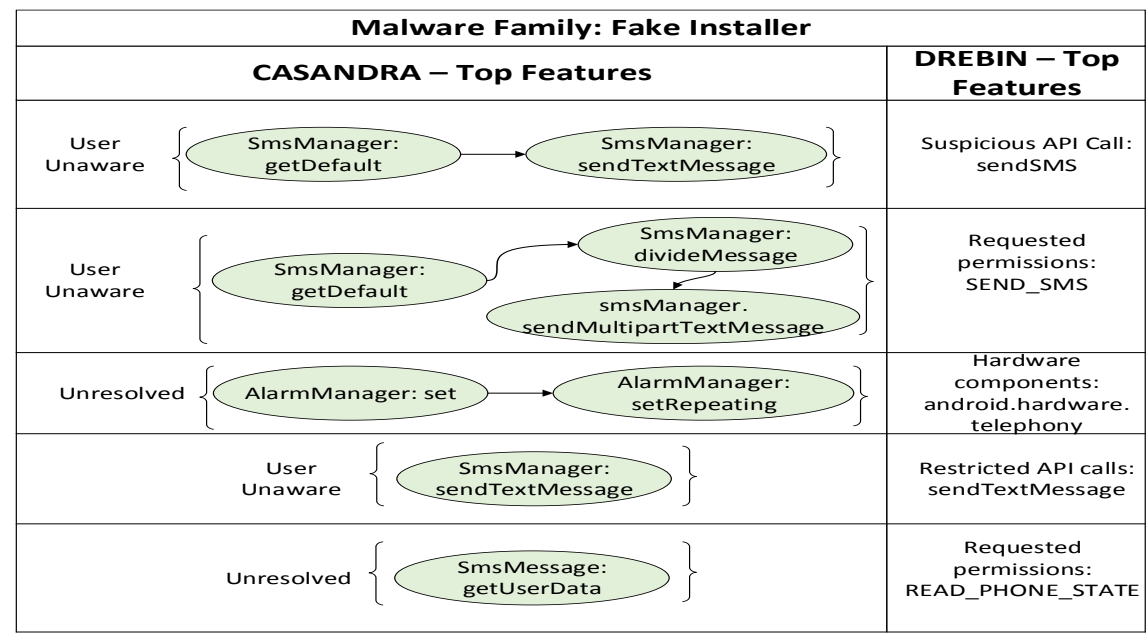

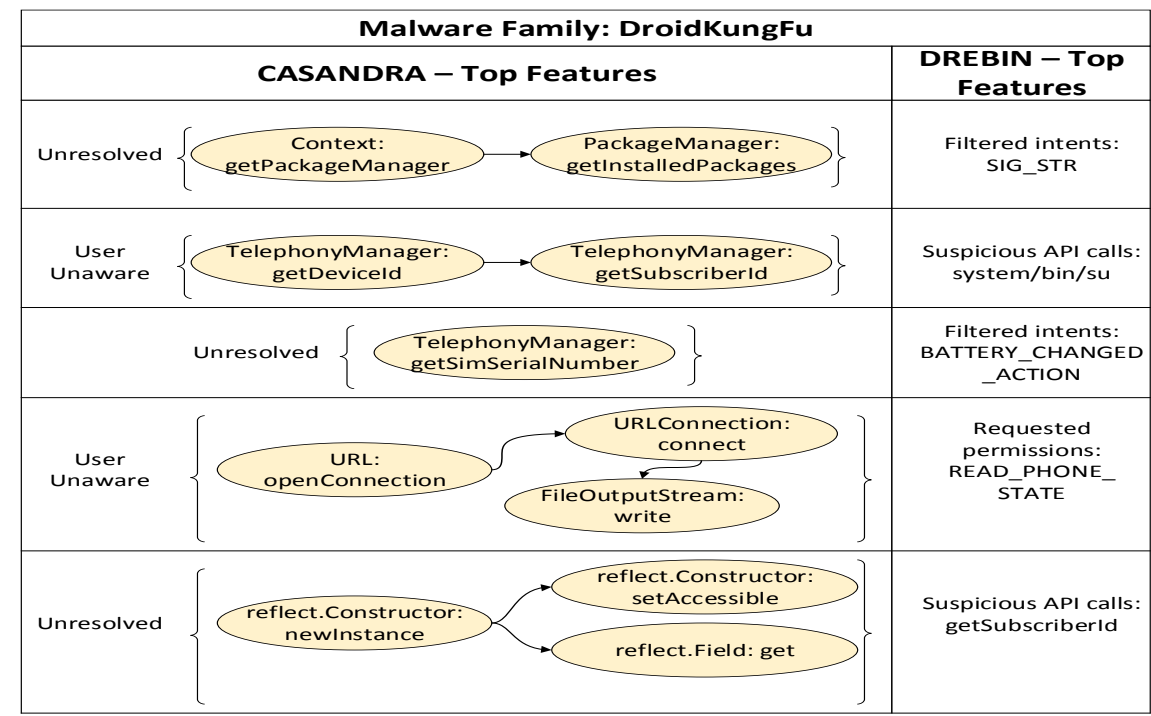

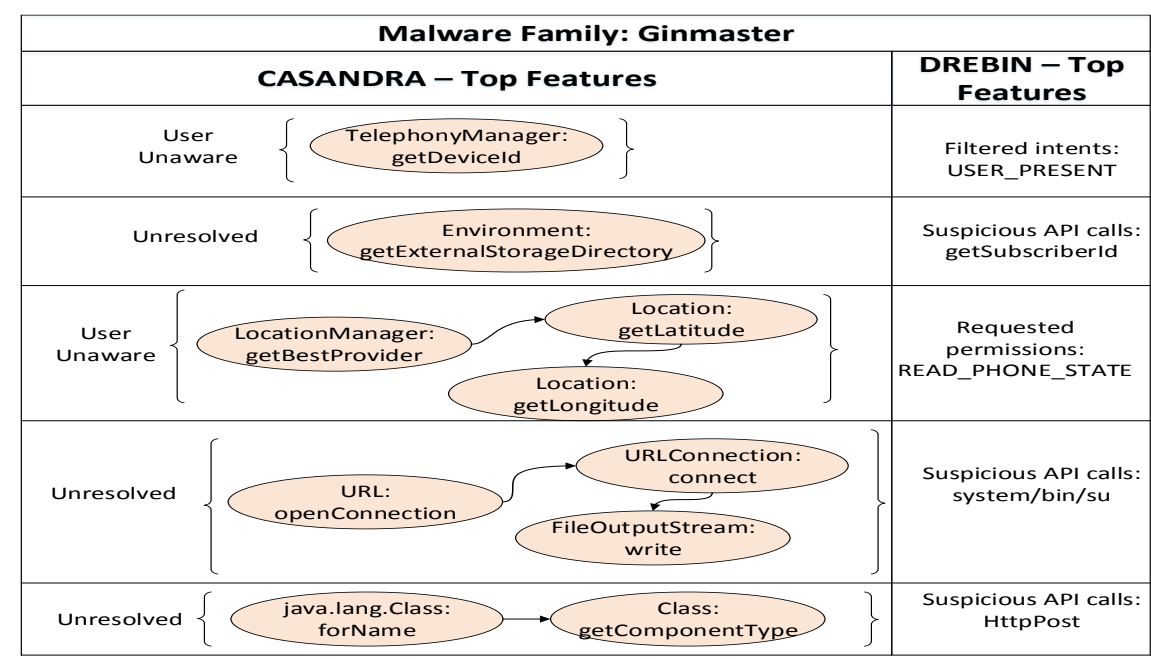

Figure 4.5: Casandra Vs. Drebin: Comparing explainability on three popular families from the DR dataset 
dominantly in the user-unaware or unresolved context. These operations include getting the default SMS manager, sending normal and multi-part SMS. Also, from the third top feature, one could see that FakeInstaller triggers these operations using AlarmManager APIs which are invoked in the background without the users' knowledge. On the other hand, DreBIN's explanations are simpler and naive. For instance, as DrebIN claims simply sending SMS does not make an app malicious. However, doing the same without the users' consent does. This distinction is not evident from DreBIN's explanations.

DroidKungFu is a sophisticated command-and-control $(\mathrm{C} \& \mathrm{C})$ based family of malware capable of exploiting several vulnerabilities in earlier version of Android to gain root access and steal sensitive data from the device. Its intention to leak a variety of private information such as unique identifiers (e.g., IMEI, IMSI) and contents from content provider (e.g., contacts) to the C\&C server over internet is adequately revealed by CASANDRA's features. Also, this malware reads system state changes such as SIG_STR through respective intents and use them to trigger malicious services in the background. Again, compared to DREBIN, CASANDRA's explanations are closer and more descriptive of the secretive operations of DroidKungFu. Interestingly, the fact that DroidKungFu leverages on Java reflection to obfuscate its attack is evident from CASANDRA's explanations.

Ginmaster is a popular Trojan family that involves in leaking users' private information to remote $\mathrm{C} \& \mathrm{C}$ servers, besides attempting to gain root access. Similar to DroidKungFu, Ginmaster performs its leaks through services that are triggered by background events such as B00T_COMPLETED. Also, these malware use reflection (through java.lang. Class APIs) to obfuscate their attacks. Again, a significant part of this behavior can be better reconstructed just by looking at the top features of CASANDRA. 


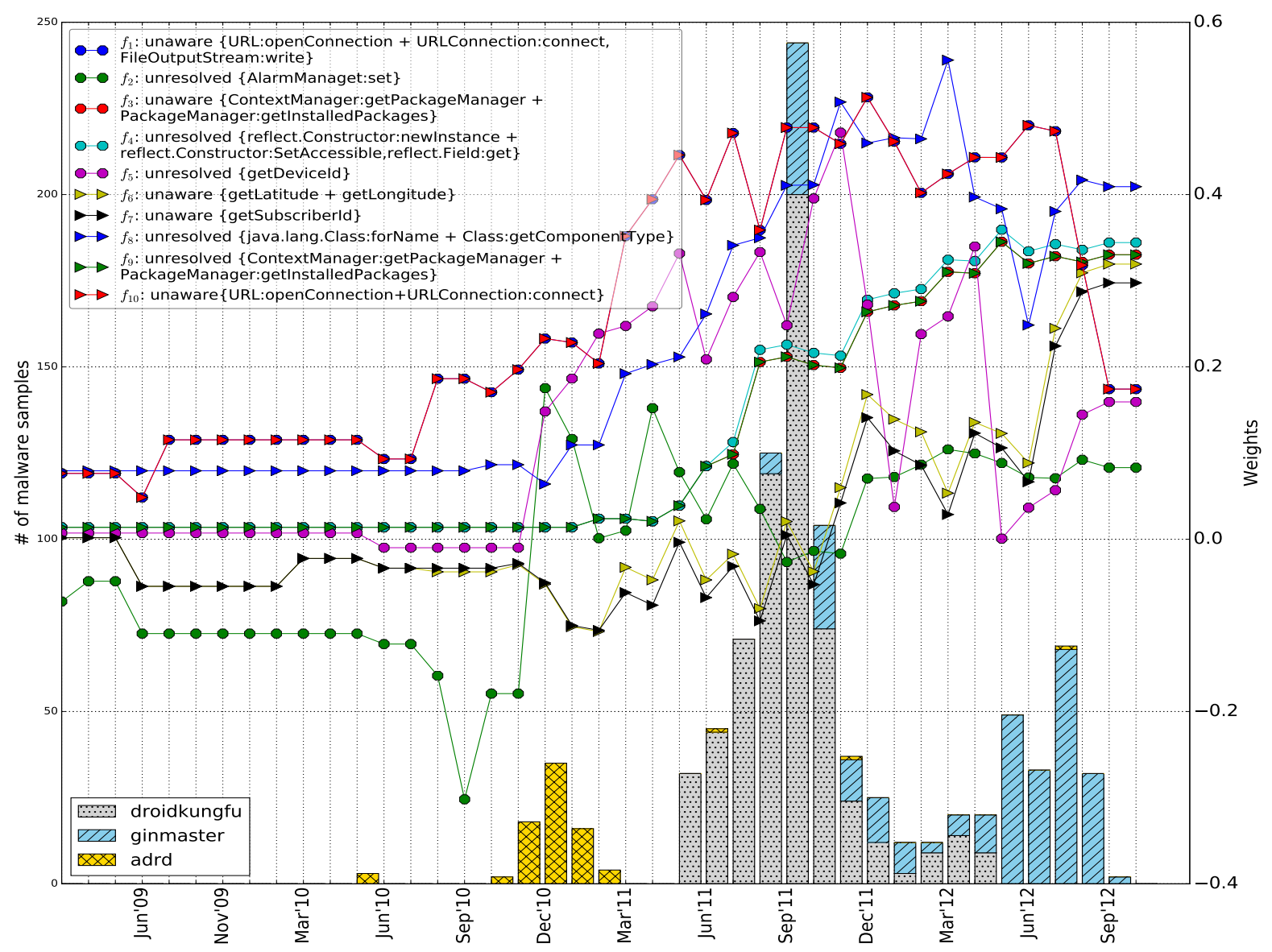

Figure 4.6: Illustration of how CASANDRA adapts to the malware evolution and population drift in the DR dataset. The $\mathrm{X}$ axis shows the distribution of family-specific malware samples. $\mathrm{Y}$ axis (left) shows the number of samples and $\mathrm{Y}$ axis (right) shows the weights of individual features. The legend contains CWLK subgraph features (format: $<$ feature id $>$ : $<$ context $>\{<$ root-node $>+$ $<$ neighbor-list $>$ \})

\subsubsection{RQ4: Adaptiveness}

In this RQ, we intend to study how CASANDRA adapts itself to malware evolution and population drift. Specifically, we intend to explore how online learning helps Casandra to learn fresh patterns and unlearn obsolete patterns of malicious behavior over time. As stated earlier, all the existing Android malware detection approaches (incl. DrebIN [25] and CSBD [26]) are based on batch learning and are not capable of adapting themselves to population drift. Adaptiveness is CASANDRA's unique feature and hence we could not 
compare this with any existing technique. Hence, we just illustrate how CASANDRA achieves adaptiveness in this subsection.

Dataset \& Experiment. In this RQ we prefer the DR dataset for three reasons: (1) it hosts malware collection over a longer period which is ideal to study malware evolution (2) its heterogeneity - it contains malware with attacks as simple as sending premium-rated SMS to complex botnets, (3) availability of accurate malware family labels.

In this experiment, the apps in DR dataset are sorted according to their month of creation. We observe that several malware families in this dataset emerge, flourish and fadeaway over time due to various domain-specific reasons. For example, DroidKungFu family first emerged in May'11 and reached its peak spread during Sep-Oct'11 and gradually disappeared by May'12.

Any good malware detector must adapt to such evolution. To examine whether CAsAnDRA exhibits this adaptiveness, we train it in an online fashion on the DR dataset following a procedure similar to RQ1 (\$4.5.1). The training and prediction quantum changes from days in RQ1 to months in this RQ.

Once the prediction and learning for all the samples in a month $m$ is over, we record the weights of all the features. Since DR dataset hosts malware collection over 44 months, the weights of each feature are recorded at 44 points in time. This gives us an opportunity to monitor and track the fluctuations in the importances of individual features over time. In general, features that characterize typical malicious and benign behaviors will be assigned large positive and negative weights, respectively. Features that do not reveal anything about malice or benignity will be assigned near-zero weights.

For a detector that adapts well to the evolving trend in malware population, the feature weights should follow the pattern of population drift. More specifically, when a particular family of malware $\mathcal{F}$ gains prominence over a period, say from month $m$ to $m^{\prime}$, the weights of features that characterize the attacks and evasion strategies of $\mathcal{F}$ should increase or 
remain high. Once the samples from family $\mathcal{F}$ start to dwindle, the weights of those features should decrease or remain low.

Results \& Discussion. In order to study this behavior, we chose three malware families that perform privacy leak attacks, namely, DroidKungFu, Ginmaster, and ADRD. The behaviors of DroidKungFu and Ginmaster are explained in the previous RQ. ADRD also secretively leaks users' private data in a similar fashion. As shown in Fig. 4.6, in the DR dataset, $A D R D$ first emerged in May'10 and gained prominence from Oct'10 to Dec'10. It finally vanished from Mar'11. A very small number of $A D R D$ samples resurfaced in Jun'11, Nov'11, and Jul'12. The evolution of DroidKungFu and Ginmaster populations could be inferred in a similar fashion from Fig. 4.6. Now, leveraging on CasandRa's explainability, we choose 10 features that best explain the privacy leaks from these families and study fluctuations in their weights over time. These fluctuations are also shown in Fig. 4.6.

One could clearly see that the feature weight fluctuations follow the evolution of the corresponding families. For instance, from Mar'09 to Apr'10 many of these features had no significant positive weights as none of these populous privacy leak families emerged. Some features had near-zero weights indicating that they do not characterize any malice at that point in time. After May'10, a small number of features that characterize ADRD start to gain positive weights. After the voluminous influx of DroidKungFu in May'11, almost all the features started to gain larger positive weights. In particular, one could see the following interesting patterns:

- Feature $f_{2}$ typically characterizes the $A D R D$ family. The weights of this feature surge rapidly during $A D R D$ 's peak spread period (i.e.,Oct-Dec'10).

- Features related to reflection (i.e., $f_{4}$ and $f_{8}$ ) and inferring installed apps (i.e., $f_{3}$ and $f_{9}$ ) are common for both DroidKungFu and Ginmaster. These features receive significant increase in their weights once DroidKungFu emerges in Jun'11. Even after DroidKungFu 
vanishes, these features continue to accumulate more weights as Ginmaster samples keep streaming till Sep'12.

- The weights of features that characterize common malicious behaviors across many families such as URL features (i.e., $f_{1}$ and $f_{10}$ ) remain predominantly high throughout the entire duration.

- Similar subgraph features exhibit very close or exactly same weight fluctuation patterns. For instance, the two PackageManager features (i.e., $f_{3}$ and $f_{9}$ ) and two URL features (i.e., $f_{1}$ and $f_{10}$ ) have exactly same pattern over the entire duration. This reflects the fact that these features characterize either same or very similar attacks.

- Finally, when we reach the end of the dataset collection period in Sep'12, we could see Casandra has assigned large positive weights to all the 10 features. Meaning, it has learned that all of them characterize malicious behaviors. In particular, CASANDRA has learnt that features such as reflection and PackageManager APIs $\left(f_{3}\right.$ and $\left.f_{9}\right)$ characterize strong malicious behaviors than other features such as reading device ID $\left(f_{5}\right)$, which may be common in benign apps as well. This is reflected from the fact that it has assigned larger positive weights to the former set of features than the latter.

In summary, we could clearly see that CASANDRA when trained in an online fashion, adapted well to the population drift and evolution in malware. So far, none of the existing techniques (incl. DreBin and CSBD) demonstrated this capability. We believe adaptiveness is the cornerstone for the practical success of ML based malware detector.

\subsection{Related Work}

Many well-known malware detection solutions have been reviewed in the previous sections. We throw light on remaining works and contrast them from CASANDRA in this subsection. Features used for detection is the most important aspect of ML based malware detectors. 
Hence, we discuss the related work with respect to this aspect.

Crowdroid [60] leverages on dynamic analysis and uses Linux system-call sequences as features. DroidAPIMiner [41] considers a hand-crafted set of sensitive APIs (along with their parameters) and package level information as features. MAST [39] uses selected permissions, Intent filters, the existence of native code and zip files, then applies Multiple Correspondence Analysis to perform malware detection. Peiravian et al. [42] take a simpler approach by considering permissions and API calls as features. CHABADA [43] leverages on text-mining techniques to study the relevance between apps' behavior and their description and henceforth detect suspiciously behaving apps. Sahs and Khan [38] extract a variety of features including permissions and CFG signatures and perform early-fusion of those features. With these features, they take an anomaly detection approach using a One-Class Support Vector Machine to detect malware. Adagio [32] constructs apps' CGs and uses byte-code instructions to assign labels to nodes. Subsequently, it captures structural information from CGs using NHGK and uses kernel SVM to detect malice. DroidMiner [31] proposes a two-tiered behavior graph to model malicious program logic into a vector of threat modalities, and then applies classification according to these modalities. Mass Vet statically analyzes apps' UI code to extract a graph that expresses the UI state and transitions. Subsequently, it uses a centroid-based approach to detect repackaged apps and DiffCom analysis to identify the malicious portions of those apps.

Recently, several techniques such as MARVIN [44], HADM [46] and StormDroid [47] adopt a hybrid approach by combining both static and dynamic analysis features and demonstrated achieving better accuracies than solutions leveraging on one of the analysis paradigms.

However, all these solutions are based on batch-learning and they neither account for concept drift in Android malware nor exhibit adaptiveness. On the contrary, CASANDRA uses online learning and is naturally adaptive to concept drift. Moreover, none of the above- 
mentioned techniques exhibit all the four qualities that CASANDRA possess (i.e., accurate context-aware detection, efficiency, explainability and adaptiveness). The only other work that discusses concept drift in Android malware is Prescience [50]. It detects drifts in malware concept using Venn-Abers predictors and retrains the computationally heavy models such as XGBoot and ExtraTrees periodically. Though, this retraining approach addresses concept drift, as we showed in $§ 4.5 .2$ it is significantly less efficient than using online learning.

\subsection{Limitations}

Static Analysis Limitations. The previous evaluation demonstrates the efficacy of our method in detecting recent malware on the Android platform. However, CASANDRA, cannot generally detect all sorts of malicious behaviors, as it builds on concepts of static analysis and lacks dynamic inspection. In particular, transformation attacks that are non-detectable by static analysis (e.g., attacks based on reflection and bytecode encryption could go undetected). To alleviate the absence of a dynamic analysis, CASANDra extracts API calls related to obfuscation, reflection, and loading of code, such as reflect. Constructor and DexClassLoader. loadClass. These features enable us to at least spot the execution of hidden code- even if we cannot further analyze it. In combinations with other features, CASANDra is still able to identify malicious behaviors despite the use of some obfuscation techniques.

Adversarial attacks. Another limitation which follows from the use of ML is the possibility of attacks by adversaries such as poisoning (see $[66,90])$. While common obfuscation strategies, such as identifier renaming and code reordering do not affect Casandra, adversaries may succeed in reducing its accuracy by incorporating benign CADG subgraph features or fake invariants into malicious apps. When attackers succeed in tampering online learning methods and make them adapt to carefully crafted noise, the damages caused are 
much worse compared to batch learners as they may continuously fit to noise allowing malware to go undetected. Even though such adversarial attacks against ML based detectors cannot be ruled out in general, meticulous sanitization of training data (see [66]) can limit their impact.

\subsection{Conclusion}

In this chapter, we present CASANDRA, an online learning based Android malware detection framework. CWLK, a novel graph kernel that facilitates capturing apps' security-sensitive behaviors along with their context information from dependency graphs is also proposed. CWLK supports explicit feature vector representation of apps' dependency graphs using which an online classifier is trained to detect malware. Our large-scale evaluations on both recent real-world and benchmark datasets demonstrate that CASANDRA outperforms two state-of-the-art techniques. CASANDRA achieves $89.92 \%$ accuracy on a real-world dataset with more than 87,000 apps outperforming state-of-the-art techniques by more than $25 \%$ in their typical batch-learning setting. On average, CASANDRA takes 28.23 microseconds to predict the label of a given sample in our large-scale experiment, which is comparable to state-of-the-art techniques, making it scalable enough to perform market-scale analysis. This superior performance and scalability make CASANDRA, in particular, and online learning based solutions, in general, better candidates for the malware detection task. 


\section{Chapter 5}

\section{A Multi-view Context-aware Approach}

\section{to Android Malware Detection and}

\section{Malicious Code Localization}

In this chapter, MKLDROID framework that is capable of performing a comprehensive multi-view malware detection and malicious code localization is presented. All the contents of this chapter are accepted as a paper titled "A Multi-view Context-aware Approach to Android Malware Detection and Malicious Code Localization” by Springer Empirical Software Engineering Journal.

\subsection{Introduction}

The CASANDRA framework presented in the previous chapter is specifically designed to handle population drift by leveraging on online learning paradigm. However, it captures only one set of features from PRGs i.e., API dependencies. This allows Casandra to capture only one semantic facet of apps and could prevent it from detecting attacks that are not very much evident through the API dependency perspective. Given the fact that PRGs are rich semantic representations of apps, in the chapter we intend to explore whether account- 
ing for features other than API dependencies could improve the accuracy.

To this end, we briefly introduce prominent single-view malware detection approaches (similar to CASANDRA) from literature and discuss the challenges that they pose, along with some relevant concepts.

Repackaged malware. It could be seen that Android app packaging and distribution model offers painless and straightforward opportunities for attackers to piggyback (i.e., inject) their malice code on benign apps which could then be spread through several thirdparty markets. Many empirical studies [34, 70-72] manifest that an overwhelming majority of Android malware are nothing but repackaged versions of benign apps. In fact, more than $80 \%$ of samples in a well-known primeval Android malware dataset named GENOME [24] are repackaged apps. Therefore, peculiarly, in these mobile malware, a predominant portion of code remains benign and only a small portion is attack related. This humongous volume, growth rate and evolution of repackaged and other types of malware have led to research community to propose several PRG based semantic malware detection approaches $[23,25,26,28,29,29,31,32,32,38,46,48-50,58,70-72]$.

The efficacy of such approaches depends primarily on the features that they extract from PRGs. Prominent robust approaches from literature have used a variety of features such as API sequences [28, 29, 31], permissions used [25, 41], information flows observed [58], instruction sequences used [32] and CFG patterns [26, 27] to learn discriminatory functions that could differentiate malware and benign apps. Understandably, each of these features represent a unique perspective (interchangeably referred as view) of apps, having their own merits and limitations.

\subsubsection{Challenges in Single-perspective Malware Detection}

Remember, the general challenges with ML based malware detection such as accuracy and scalability have been discussed and addressed in the two previous chapters (chapter 3 and 
4). We now discuss remaining challenges with such approaches:

(C3.1) Integrating multiple perspectives. Each of the aforementioned feature sets (API sequences, information flows, etc.) provide a complementary perspective of the app, but no one perspective is completely sufficient to determine whether or not it is malicious. More specifically, some views are more amenable to detect certain attacks while they may not characterize several other attacks well. For instance, information flow features are amenable for detecting 'privacy leak' attacks but are incapable of revealing 'privilege escalations'. A majority of existing approaches use either one or a selected few of the aforementioned views but not all of them. This prevents them from detecting a substantial majority of attacks.

(C3.2) Malicious code localization. In general, almost all the ML based approaches $[25-29,31-33,38,43,46-48,50,58-60]$ act as holistic black-box solutions as they just predict whether or not a given app (as a whole) is malicious. Such approaches are incapable of locating malice code portions (e.g., classes/methods containing such code). In general, precise malicious code localization would not only help to assess the approaches' trustworthiness but also cater several important applications such as supporting human analysts studying malware behaviors, engineering malware signatures and other counter-measures. Malicious code localization becomes particularly useful in the case of repackaged malware, as a predominant portion of their code remains benign and only a small portion is attack related.

\subsubsection{Our Approach}

We take these challenges into consideration and propose MKLDRoID, a unified framework which integrates all the above mentioned five perspectives, namely, API dependencies, permission dependencies, information source and sink dependencies, instruction sequences and CFG patterns to perform effective detection. Furthermore, leveraging on its multi-view 
analysis, it precisely locates malicious code portions in apps. In particular, MKLDRoID is developed with the three following design goals:

1. Accuracy. Accuracy of MKLDroID, which is a multi-perspective PRG based approach depends on two factors: (i) how well it retains PRG's expressiveness and (ii) how effectively it integrates different perspectives (i.e., how challenge C3.1 is addressed). To address expressiveness challenges, we leverage on our previous work and use CWLK that is specifically designed to perform accurate malware detection by capturing both structural and contextual information from PRGs. To address C3.1, we resort to MKL [91], a wellknown principled approach to integrate multiple feature sets with different modalities. To the best of our knowledge, ours is the first approach that captures contextual and structural information from different dependencies that emanate from PRGs representing different semantic perspectives of an app.

2. Efficiency. MKLDroID achieves its efficiency through the combined use of a scalable graph kernel (i.e., CWLK) and an efficient MKL approach, namely, SMO-MKL [92].

3. Locating malice code. Besides effective detection, a salient feature of MKLDROID is its ability to locate malicious code portions in PRGs. We achieve this by meticulously extracting explainable features from each perspective and combining them in an interpretable manner. To this end, we propose a method for training a MKL SVM in the dual formulation and subsequently switching to primal formulation for prediction and interpretation. This process allows MKLDRoID to assign a maliciousness scores (m-scores, for short) to each node in the PRG which are then aggregated to arrive at m-scores of methods and classes that encompass them. To the best of our knowledge, ours is the first approach that performs such fine-grained malicious code localization without requiring any apriori information on apps' piggybacking or composition. This, in effect, addresses challenge C3.2.

Experiments. MKLDroID is evaluated through large-scale experiments on more than 60,000 apps from benchmark datasets and collected in-the-wild. On benchmark datasets, 
MKLDRoID achieves more than 97\% F-measure, which is comparable to state-of-the-art approaches. More importantly, in an experiment closer to the real-world setting where the test-set is historically posterior (nearly by 2 years) to the training set, MKLDRoID achieves $71 \%$ F-measure outperforming state-of-the-art techniques by $11 \%$. On recent wild apps, it achieves $72 \%$ F-measure, outperforming state-of-the-art approaches by $8 \%$. In all these experimental settings, MKLDRoID maintained better efficiency than two state-of-the-art techniques. In malicious code localization experiments, our approach, on average, located more than $94 \%$ of all malice classes within its top 10 classes with highest m-scores.

Contributions. In this chapter, we make the following contributions:

- We propose MKLDroid, a novel malware detection framework which integrates these views using MKL thereby extracting a semantically richer representation. This helps MKLDRoID achieve superior accuracies over approaches which use either one or a selected few of the perspectives.

- We propose a novel kernel methods approach to locate and rank malicious code portions (i.e., methods/classes) in PRGs. To the best of our knowledge, ours is the first approach that performs automatic multi-view malicious code localization.

- We contribute to future research on malicious code localization by releasing MKLDroID's results. M-scores for all basic blocks, methods and classes from apps in the benchmark datasets, Drebin [25] and Mystique [96] are made available at: https: //sites.google.com/site/mkldroid.

The remainder chapter is organized as follows: We begin by introducing the background and motivations for our framework’s design in $\$ 5.2$. The proposed MKLDROID framework is presented in $§ 5.3$. The experimental design and implementation details are furnished in §5.4. Evaluation results and discussions are presented in section §5.5. Related work, limitations and conclusions are provided in $§ 5.6, \S 5.7$ and $\S 5.8$, respectively. 


\subsection{Motivations}

Remember that motivations for performing context-aware Android malware detection (i.e., capturing both contextual and structural features from PRGs) is presented in chapter 3 already. We now discuss the motivations for performing multi-view detection.

To illustrate the necessity to integrate multiple perspectives, we consider a complex and more recent variant from the Geinimi family which was introduced in $\$ 3.2$. Besides leaking the geographic location, this variant leaks other sensitive information such as device identifiers and contacts. The (simplified) malicious code portion that is responsible for these activities is shown in Fig. 5.1 (a). Firstly, the malware reads the geographic location and sends this information to a remote server, which acts as the command-and-control (C\&C) server, thereafter (lines 8-9). Subsequently, the malware reads the users' contacts (i.e., names and phone numbers) from the content provider database and sends it to the $\mathrm{C} \& \mathrm{C}$ server along with the IMEI number that serves as a unique identifier for the victimized device (lines 16-39). This malicious privacy leak comprises of a variety of actions that involves using APIs and URIs that are related to permissions, information sources and sinks (viz. openConnection, getDeviceId, Contacts.CONTENT_URI, Phone.CONTENT_URI) and other security-sensitive APIs (viz. getLatitude, getLongitude, getContentResolver, query).

Through statically analysing the app, the graphs in Fig. 5.1 (b) to (g) are constructed. While the formal definitions and detailed explanation of these graphs are provided later in §5.3.2, brief explanations are provided here to motivate capturing multiple perspectives of an app through them.

The ICFG corresponding to the aforementioned code is shown in Fig. 5.1 (b). The basic blocks (i.e., is a sequence of instructions in a method with only one entry point and one exit point which represents the largest piece of the program that is always executed altogether) 


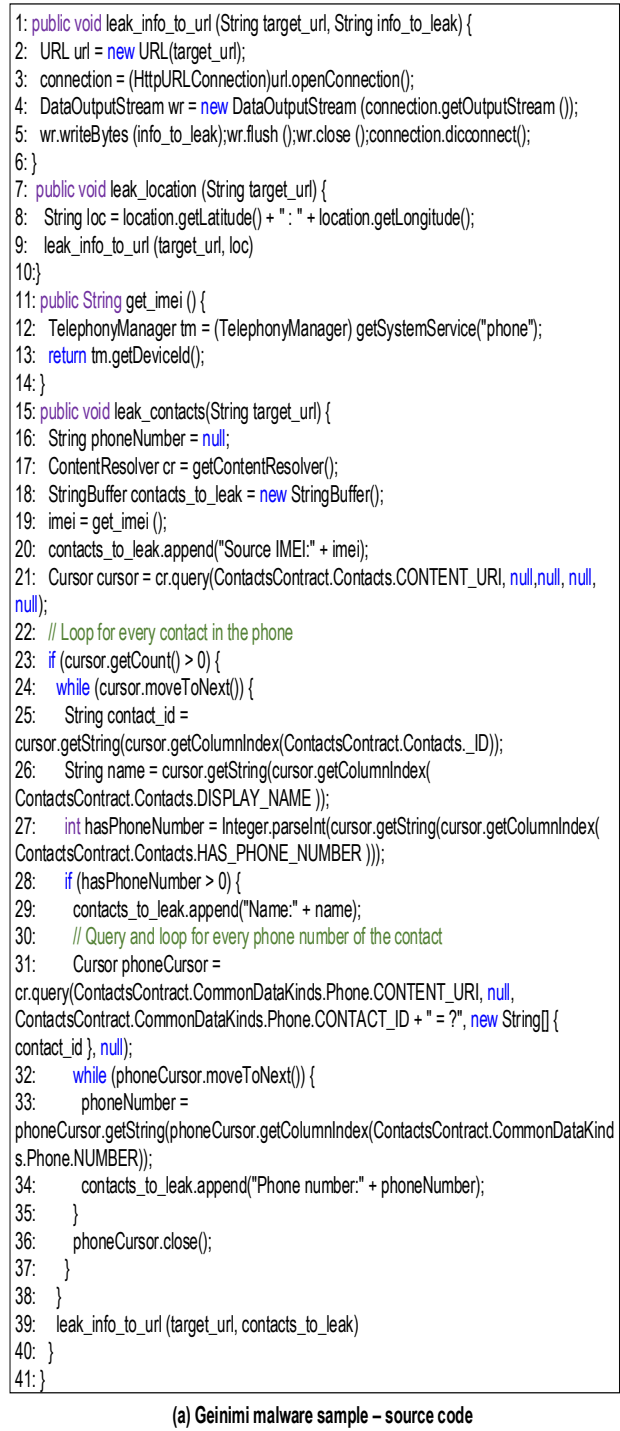

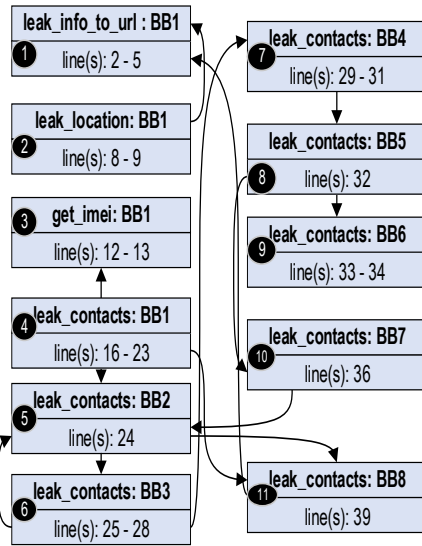

(b) ICFG
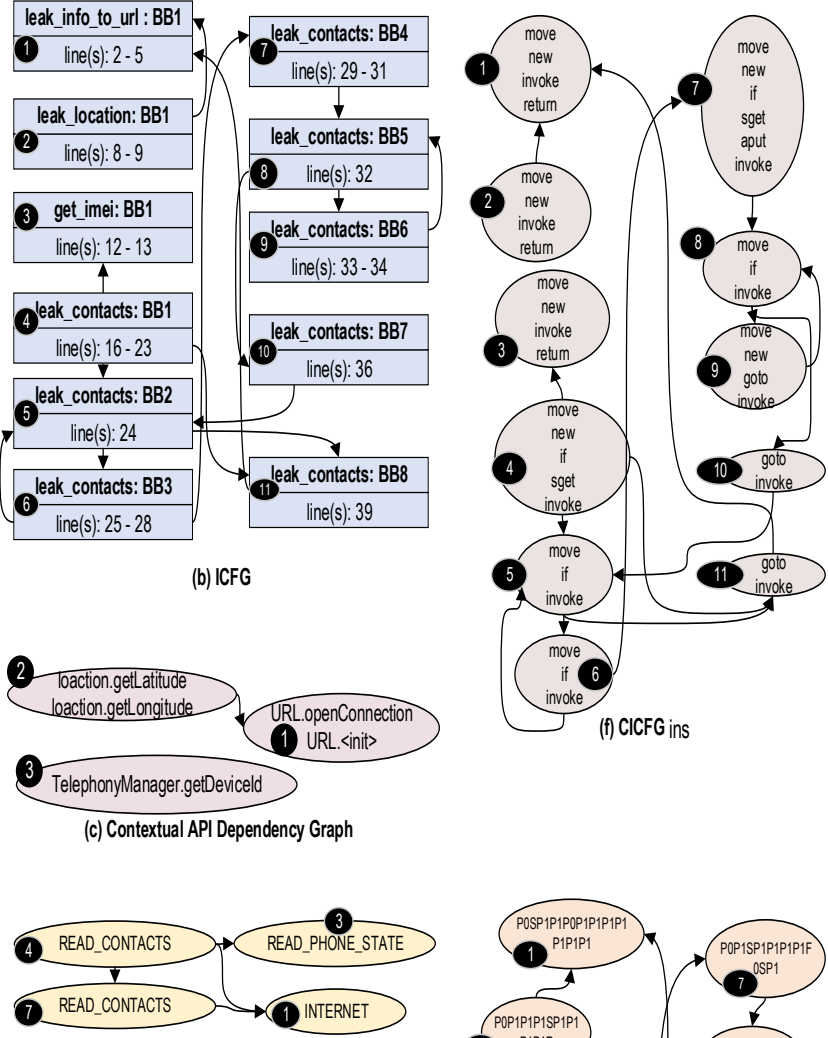

(d) Contextual Permission Dependency Graph

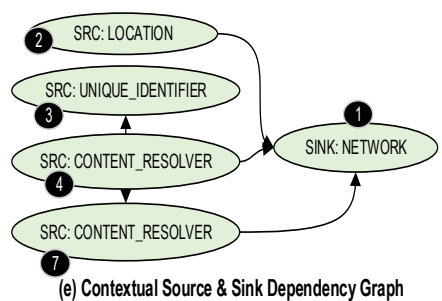

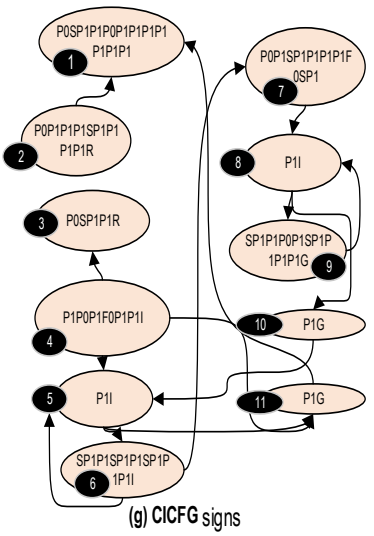

Figure 5.1: Extracting different five semantic perspectives of the Geinimi variant. (a) code snippet corresponding to leaking location, IMEI and contacts. (b) ICFG representation of the code. (c) Contextual API Dependency Graph corresponding to the code snippet. (d) Contextual Permission Dependency Graph. (e) Contextual Source \& Sink Dependency Graph. (f) Contextual ICFG with Dalvik bytecode instructions used as node labels. (g) Contextual ICFG with textual CFG signatures used as node labels. 
of methods form the nodes of the ICFG and the edges denote the control flow across these nodes. The node headers indicate the method name and the basic block number. The node content specifies the line numbers of code instructions belonging to the basic block. Subsequently, the context under which each ICFG node is reached is determined and added as a node attribute to obtain the CICFG. In this particular case, all ICFG nodes are reached in the user-unaware context. In sum, CICFG represents the control flow structure of the code in a precise and granular manner along with the contextual information.

Abstraction to arrive at multiple perspectives. Having, constructed CICFG of an app, one way to detect malicious behavior is to abstract the functionalities performed by its nodes and subsequently, detect the contextual subgraph patterns that correspond to such behaviors. We precisely follow this approach and propose five different abstractions of CICFG, each of which represent a semantic view of the app. They are, CADG (defined previously in §4.3.2), Contextual Permission Dependency Graph (CPDG), Contextual Source \& Sink Dependency Graph (CSSDG), $\mathrm{CICFG}_{\mathrm{ins}}$ : CICFG nodes labeled with the Dalvik instructions that they access and $\mathrm{CICFG}_{\text {signs }}$ : CICFG nodes labeled with structured control flow signatures. These graphs for the Geinimi example are depicted in Fig. 5.1 (c) to (g).

The CADG in Fig. 5.1 (c), is obtained by considering only the CICFG nodes that access security-sensitive Android APIs. All other nodes are removed and paths that exists between these nodes in the CICFG conditionally become edges in CADG. The CADG nodes are labeled with the sensitive APIs that they access. Similarly, we take into consideration the permissions, information sources/sinks and Dalvik instructions accessed in every CICFG node to produce CPDG, CSSDG and $\mathrm{CICFG}_{\text {ins }}$ respectively. Lastly, $\mathrm{CICFG}_{\text {signs }}$ is obtained by labeling every CICFG node with a textual signature that represents basic block's control flow structure. A control-flow analysis encoding grammar proposed by Cesare and Xiang in [21] is used to infer these textual signatures.

Complementary nature of the perspectives. With all these graphs constructed, it 
could be seen from Fig.5.1 (c) that considering only the API dependencies, we could capture the behavior corresponding to leaking location information and IMEI number. However, behavior corresponding to leaking the contacts could not be detected. Similarly, considering only the permission dependencies (see Fig.5.1 (d)), we could capture the behaviour corresponding to leaking the contacts but not the one corresponding to location information. Considering the dependencies among information sources and sinks, captures both the aforementioned leaks but fails to capture leaking IMEI (see Fig.5.1 (e)). Also, CSSDG may not capture common malicious behaviours such as gaining root access or installing additional apps as they do not involve sources or sinks [58]. $\mathrm{CICFG}_{\text {ins }}$ characterizes apps through the structural dependency among Dalvik instructions. For instance, the privacy leaks in the example is characterized in a sequence involving nodes with move and invoke instructions. $\mathrm{CICFG}_{\text {signs }}$ characterizes apps based on the control-flow structure of the code. This helps to model unusual control flow jumps, heavy usage of junk/unwanted instructions and complex loops which are predominant in malware $[21,26]$.

In summary, each of these graphs represent different perspectives of a given app, capturing information at different levels of granularity with different modalities. While, each of these perspectives are capable of capturing certain characteristics of malware, they fail to capture certain other important characteristics due to their inherent limitations. We hypothesize that a much comprehensive and accurate malware detection model could be constructed by appropriately combining information from all these perspectives. Driven by this motivation, we construct a unified malware detection framework that is able to systematically integrate all the aforementioned views in the hope that, while a malware can disguise itself in some views, disguising itself in every view while maintaining malice intent will prove to be more strenuous. 


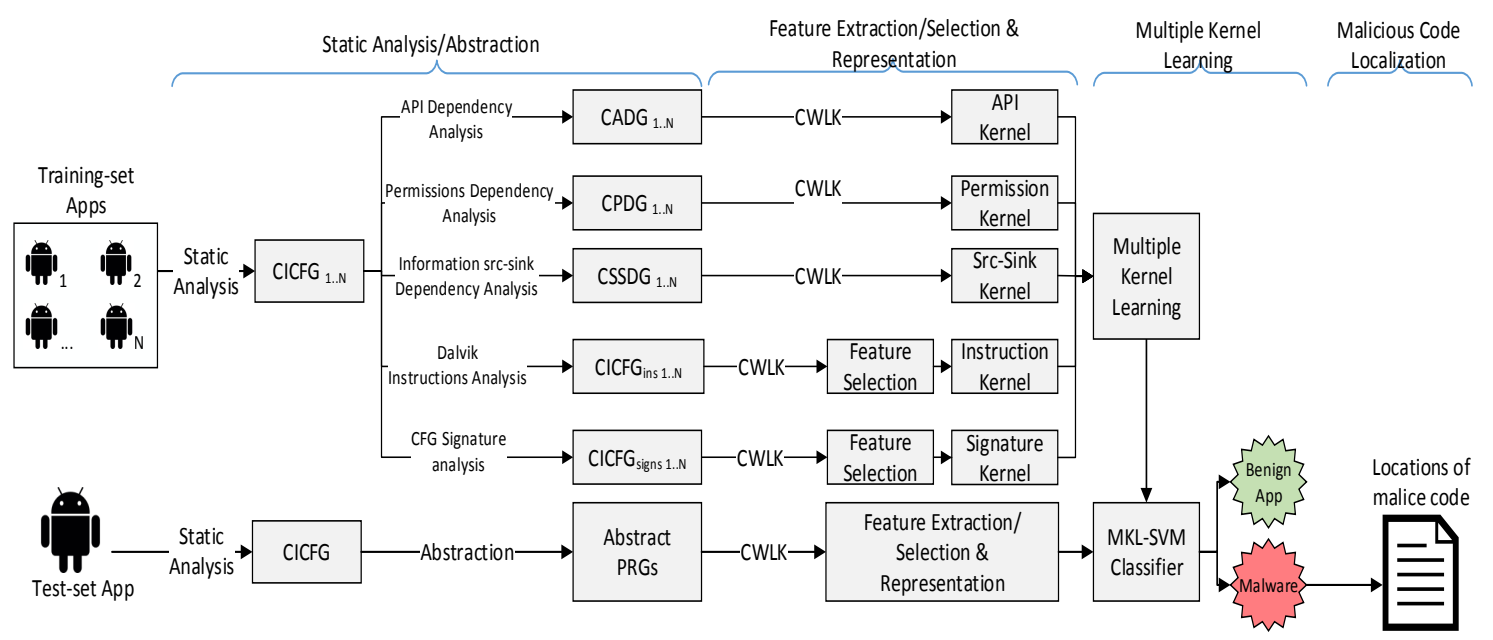

Figure 5.2: MKLDRoID - Overview

\subsection{Methodology}

The methodology of MKLDRoID framework designed to perform multi-perspective, context-aware malware detection and malicious code localization is presented in this section. We begin by describing the framework overview and subsequently, explain each component of the framework in separate subsections.

\subsubsection{MKLDROID - Framework Overview}

As with any ML based framework, MKLDRoID has a separate model building (training) phase and evaluation (testing) phase. In the training phase, a set of known malware and benign apps are used to build the detection model. In the testing phase, the model is evaluated for its capability to automatically detect previously unseen malware. The overview of MKLDRoID framework is presented in Fig. 5.2. The framework has four components as described below.

1. Static Analysis. To begin with, we perform static analysis on a given app and construct its CICFG. Subsequently, we construct the five above mentioned PRGs, namely, CADG, CPDG, CSSDG, $\mathrm{CICFG}_{\text {ins }}$ and $\mathrm{CICFG}_{\text {signs }}$ each of which represent a unique per- 
spective of the app. This procedure is explained in detail in $\$ 5.3 .2$.

2. Feature Extraction, Selection and Representation. Our framework considers contextual subgraphs from these five PRGs as semantic features to perform malware detection. Hence, after these PRGs are constructed, those subgraphs which represent securitysensitive events that happen in an app along with their context(s) are extracted as using CWLK. It yields separate feature vector representations for each PRG. In some cases when the dimensionality of these vectors are large, we use feature selection to reduce them. The detailed feature extraction, selection and representation procedure is explained in §5.3.3.

3. Multiple Kernel Learning. After representing each of the PRGs as vectors, we need to appropriately combine them to build a single ML classifier that thoroughly leverages the strengths of individual perspectives and remains robust to their weaknesses. In other words, the perspectives have to be combined in a way that they complement each other and enhance the prediction accuracy. MKL [91], provides a principled way to facilitate learning from this multi-view, multi-granular and multi-modal data. Therefore, we use SMO-MKL [92], a well-known MKL algorithm, to combine representations from all these PRGs and train an SVM to perform malware detection. The detailed procedure is explained in \$5.3.4. Subsequent to this training MKLDRoID is ready to perform malware detection at scale.

4. Locating Malice Code. During evaluation, if MKLDroID predicts a sample to be malware, it further locates and reports the nodes in the CICFG (i.e., basic blocks) that perform malice operations and end up contributing significantly to the final prediction. That is, each CICFG node is assigned an $\mathrm{m}$-score that quantifies the statistical significance of malice operation(s) it is involved in. The m-scores of basic blocks are aggregated to arrive at the same of their encompassing methods and classes. In most cases, nodes with high m-scores reveal the app's characteristics related to its malice behaviors. The detailed procedure is presented in $\S 5.3 .5$.

Now, each components of MKLDroID is explained in detail in the four following sub- 
sections.

\subsubsection{Static Analysis}

As a first step towards constructing the five above mentioned PRGs for a given app, we perform static control-flow analysis and construct its ICFG. Formally, an ICFG is defined as follows:

Definition 5.1 (ICFG). ICFG $=\left(N_{i}, E_{i}\right)$ for an app $a$ is a directed graph in which each node $b b \in N_{i}$ denotes a basic block of a method $m$ in $a$, and each edge $e\left(b b_{1}, b b_{2}\right) \in E_{i}$ denotes either an intra-procedural control-flow dependence from $b b_{1}$ to $b b_{2}$ or a calling relationship from $b b_{1}$ to $b b_{2}$ and $E_{i} \subseteq N_{i} \times N_{i}$.

Compared to other well-known PRGs such as CG, ICFG is a more fine-grained representation of the control flow sequence inside an app. Hence working on abstractions of ICFGs enable us to capture the finer details of apps, which are helpful in distinguishing malicious and benign behaviors with greater precision. Hence, we choose to abstract apps' behaviors from their ICFGs.

CICFG construction. Once an app's ICFG is constructed, we proceed towards identifying contexts under which every ICFG node is reached to build its CICFG. The strategy used in CASANDRA (see §4.3.2) is reused to here to identify the reachability contexts of nodes. Formally a CICFG, is defined as follows:

Definition 5.2 (CICFG). $C I C F G=\left(N_{i}, E_{i}, \xi\right)$ of an app $a$ is a directed graph in which each node $b b \in N_{i}$ denotes a basic block of a method $m$ in $a$, and each edge $e\left(b b_{1}, b b_{2}\right) \in E_{i}$ denotes either an intra-procedural control-flow or a calling relationship from $b b_{1}$ to $b b_{2} . \xi$ is a set of contexts through which every node $b b \in N_{i}$ could be reached.

Once the CICFG of an app is constructed, we abstract it with various Android platform specific analysis to construct the five different PRGs that MKLDroID leverages on. For each of the PRGs, the procedure to construct them and their formal definitions are provided 
along with relevant explanations below.

CADG construction. Intuitively, CADG of an app is obtained from its CICFG by considering only the nodes that access security-sensitive APIs ${ }^{1}$. All other nodes are removed and paths that exists between such nodes in the CICFG become edges in CADG, if they satisfy certain conditions as described in the definition below. If multiple APIs are invoked in a single CICFG node, it is labeled with the sorted list of unique APIs being invoked. CADG's formal definition is as follows:

Definition 5.3 (CADG). CADG can be represented as a 4-tuple, $C A D G=$ $\left(N_{A}, E_{A}, \lambda_{A}, \xi\right)$, where $N_{A}$ is a finite set of nodes and $n \in N_{A}$ is a basic block that accesses at least one security-sensitive API. $E_{A} \subseteq N_{A} \times N_{A}$ is a set of directed edges where an edge from $e\left(n_{1}, n_{2}\right) \in E$ exists, iff there exists a path $p\left(n_{1}, n_{2}\right)$ between these two nodes in the CICFG and $\operatorname{method}\left(n_{1}\right)=\operatorname{method}\left(n_{2}\right)$, where $\operatorname{method}(n)$ denotes the method that encompasses basic block $n^{2} . \lambda_{A}$ is the set of labels representing the security-sensitive APIs and $\ell_{A}: N_{A} \rightarrow \lambda_{A}$ is a labeling function which assigns a label to each node. $\xi$ is a set of contexts through which every node in the CADG could be reached and $C \rightarrow \xi$ is a function which assigns the context to each node.

CPDG. Similarly, CPDG of an app is obtained from its CICFG by considering only the nodes whose functionality pertains to using Android permission(s). APIs and URIs observed in nodes are used to determine their permissions pertinence ${ }^{3}$. All other nodes are removed and paths that exists between such nodes in the CICFG conditionally become edges in CPDG. If multiple permissions are used in a single CICFG node, then the same strategy followed in CADG is used label corresponding CPDG nodes.

Definition 5.4 (CPDG). Formally, $C P D G=\left(N_{P}, E_{P}, \lambda_{P}, \xi\right)$, and node $n \in N_{P}$ is a

${ }^{1}$ The strategy used in CASANDRA is reused here to identify sensitive APIs

${ }^{2}$ The same strategy followed in CASANDRA is reused here. See $\S 4.3 .2$ for justifications on translating paths between nodes in methods to CADG edges.

${ }^{3}$ PScout [51] provides a mapping from Android APIs and URIs to permissions required to access them. Furthermore, we infer the usage of intents, reflection and native code through relevant APIs and consider them as using special permissions. We use these mappings to build CPDGs. 
basic block whose functionality pertains to using permissions. $E_{P} \subseteq N_{P} \times N_{P}$ is a set of directed edges where an edge from $e\left(n_{1}, n_{2}\right) \in E_{P}$ exists, iff there exists a path $p\left(n_{1}, n_{2}\right)$ between these two nodes in the CICFG and $\operatorname{method}\left(n_{1}\right)=\operatorname{method}\left(n_{2}\right) . \quad \lambda_{P}$ is the set of labels representing the concerned permission(s) and $\xi$ is a set of contexts through which every node in the CPDG could be reached.

CSSDG. CSSDG of an app is obtained from its CICFG by considering only the nodes whose functionality pertains ${ }^{4}$ to using information sources (e.g., contacts) and sinks (e.g., network). All other nodes are removed and paths that exists between such nodes in the CICFG conditionally become edges in CSSDG.

Definition 5.5 (CSSDG). Formally, $C S S D G=\left(N_{S}, E_{S}, \lambda_{S}, \xi\right)$, and node $n \in N_{S}$ is a basic block whose functionality pertains to information sources or sinks. $E_{S} \subseteq N_{S} \times N_{S}$ is a set of directed edges where an edge from $e\left(n_{1}, n_{2}\right) \in E_{S}$ exists, iff there exists a path $p\left(n_{1}, n_{2}\right)$ between these two nodes in the CICFG and $\operatorname{method}\left(n_{1}\right)=\operatorname{method}\left(n_{2}\right) . \lambda_{S}$ is the set of labels representing the concerned source(s) or $\operatorname{sink}(\mathrm{s})$ and $\xi$ is a set of contexts through which every node could be reached.

CICFG $_{\text {ins }}$. Recently, studies such as AdAGio [32] demonstrated that structural information from PRGs labeled with Dalvik instruction categories (e.g., move, add, iget, etc.) could capture security-sensitive behaviors and thus help detecting malware effectively. Inspired by them, we extend this approach by supplementing the instruction-level structural information with contextual information. To this end, we build $\mathrm{CICFG}_{\text {ins }}$ as described below.

Definition 5.6 $\left(\right.$ CICFG $\left._{\text {ins }}\right)$ Formally, $C I C F G_{\text {ins }}=\left(N_{i}, E_{i}, \lambda_{I}, \xi\right)$, where $N_{i}, E_{i}$ and $\xi$ are the nodes, edges and node contexts in the CICFG, respectively. The function $\ell_{i}: N_{i} \rightarrow \lambda_{I}$ labels every node $n \in N_{i}$ with the categories of Dalvik instructions ${ }^{5}$ that it accesses.

\footnotetext{
${ }^{4}$ To identify information sources and sinks accessed in CICFG nodes, we leverage on SUSI [52] and MUDFLOW [58]. Together, these works map APIs and URIs to 15 source and 18 sink categories.

${ }^{5}$ To determine the categories of Dalvik instructions to be used as $\mathrm{CICFG}_{\text {ins }}$ node labels, we refer to ADAGIO [32]. The authors manually analyzed and categorized all the instructions into 15 distinct categories (such as move, invoke, etc.).
} 
CICFG $_{\text {signs }}$. Similar to Adagio, Allix et. al. [26] proposed an approach which leverages on control-flow structural information. They represent the structure of basic blocks in every method as textual signatures following a method devised by Pouik et al. [18] . This signature is an abstraction of code's structure, but discards low-level details such as variable or register names and numbers. This property is particularly desirable for malware detection as variants from same family may share the same abstract CFG while having different bytecode. Also, this helps to model unusual control flow structure such as jumps, heavy usage of junk/unwanted instructions and complex loops which are tell-tale signs of malware [16, 26, 27, 32]. Overall, using an abstract signature representation of CFG basic blocks could allow taming common obfuscations used by malware. Inspired by Allix et. al's approach, we extend it by supplementing the CFG signature-level structural information with contextual information. To this end, we build CICFG $_{\text {signs }}$ as described below.

Definition $5.7\left(\right.$ CICFG $\left._{\text {signs }}\right)$ Formally, $C I C F G_{\text {signs }}=\left(N_{i}, E_{i}, \lambda_{s}, \xi\right)$, where $N_{i}, E_{i}$ and $\xi$ are the nodes, edges and contexts in the CICFG, respectively. The function $\ell_{s}: N \rightarrow \lambda_{s}$ labels every node $n \in N_{i}$ with the control-flow signatures arrived at using Cesare and Xiang's grammar [21].

\subsubsection{Feature Extraction, Selection and Representation}

Once the five PRGs are constructed as described above, we proceed to extract and select contextual subgraph features from each of them using CWLK and represent them as vectors. Algorithm 2 discussed previously in $\S 4.3 .3$ is used for this feature extraction and representation. Given the (normalized) feature vector representations of PRGs emanating from a view, we could compute the kernel matrix corresponding to that view by taking the dot products of those vectors. This process is followed to arrive at the kernel matrices

${ }^{6}$ Pouik et al. [18] leveraged on a grammar proposed by Cesare and Xiang [21] to represent CFG textual signatures in their work on establishing similarity between Android apps. 
corresponding to every view.

Feature selection. With the kernel matrix thus obtained, an MKL classifier could be trained to detect malware. However, we note that the vocabularies of contextual subgraph features, $\mathbb{V}$, for $\mathrm{CICFG}_{\text {ins }}$ and $\mathrm{CICFG}_{\text {signs }}{ }^{7}$ are extremely large (more than 300,000 features emerge from these perspectives in all our experiments. See $\$ 5.5$ for more details). This leads to building very high dimensional embeddings which adversely affects both the accuracy and efficiency. Hence, to mitigate this, we perform feature selection over the $\mathrm{CICFG}_{\text {ins }}$ and $\mathrm{CICFG}_{\text {signs }}$ embeddings using the chi-squared feature selection algorithm [94] and then compute their respective kernel matrices. The number of features to be selected from these PRGs is empirically determined to be 5,000. This helps to retain only the informative subgraph features, thereby preventing overfitting and improving efficiency.

\subsubsection{Multiple Kernel Learning}

Once the feature vectors of all the apps in the training-set are built for all five perspectives, we train an MKL classifier. This procedure is explained below with relevant notations.

Notations. Denote the features of an app $\mathbf{x}$ corresponding to each of perspectives as following vectors: CADG: $\overrightarrow{\mathbf{x}}_{a}=\left[x_{a}^{1}, x_{a}^{2}, \ldots\right]^{T}$, CPDG: $\overrightarrow{\mathbf{x}}_{p}=\left[x_{p}^{1}, x_{p}^{2}, \ldots\right]^{T}$, CSSDG: $\overrightarrow{\mathbf{x}}_{s s}=\left[x_{s s}^{1}, x_{s s}^{2}, \ldots\right]^{T}, \mathrm{CICFG}_{\mathrm{ins}}: \overrightarrow{\mathbf{x}}_{i n}=\left[x_{i n}^{1}, x_{i n}^{2}, \ldots\right]^{T}$, Signature: $\overrightarrow{\mathbf{x}}_{s i}=\left[x_{s i}^{1}, x_{s i}^{2}, \ldots\right]^{T}$ where $x_{v}^{i}$ denotes individual features emerging from view $v$ and the set of all the views is denoted as $V=\{a, p, s s, i n, s i\}$. The label corresponding to an app $\mathbf{x}^{(i)}$ is denoted as $y^{(i)} \in\{-1,+1\}$, where -1 indicates benign and +1 indicates malicious apps. Let the total number of apps in the training set be $K$. The kernel value between a pair of apps $\mathbf{x}^{(i)}$ and $\mathbf{x}^{(j)}$ corresponding

\footnotetext{
${ }^{7}$ The reason why such an issue rises only in the case of $\mathrm{CICFG}_{\text {ins }}$ and $\mathrm{CICFG}_{\text {signs }}$ is understandable. That is, in the case of CADG, CPDG and CSSDG, the number of unique node labels is limited by the APIs, permissions, information source and sink categories available. Consequently, limited contextual neighborhood labels to emerge from the relabeling process and thereby limiting the size of the vocabulary. However, in the case of $\mathrm{CICFG}_{\text {ins }}$ and $\mathrm{CICFG}_{\text {signs }}$, the number of unique node labels (i.e., the number of unique instruction sequence and CFG signatures, respectively) across the whole dataset is extremely large, leading to mammoth vocabulary $\Sigma^{*}$.
} 
to each perspective is computed as follows:

$$
k_{v}\left(\mathbf{x}^{(i)}, \mathbf{x}^{(j)}\right)=\left\langle\overrightarrow{\mathbf{x}}_{v}^{(i)}, \overrightarrow{\mathbf{x}}_{v}^{(j)}\right\rangle
$$

where $\overrightarrow{\mathbf{x}}_{v}^{(i)}$ denotes the vector representation of $\mathbf{x}^{(i)}$ in perspective $v$ and $\langle\cdot, \cdot\rangle$ denotes dot product over a pair of vectors. Meaning, the similarity measured in view $v$ is nothing but the normalized linear kernel. Following this procedure, the kernel matrix across all the apps in the training set for each view is arrived. For the sake of simplicity, we refer to the kernel built from CADG view as API kernel. Similarly, the four remaining kernels are referred as Permission, Src-sink, Instruction and Signature kernels.

Kernel methods. Given a kernel matrix over the training samples, the goal of classical kernel-based learning with SVMs is to learn the vector, $\alpha$, describing each sample $\mathbf{x}$ 's contribution to the hyperplane that separates the points of the two classes ( $a k a$ decision boundary) with a maximal margin [95] and can be found with the following optimization problem:

$$
\min _{\alpha}\left(\frac{1}{2} \sum_{i=1}^{K} \sum_{j=1}^{K} \alpha^{(i)} \alpha^{(j)} y^{(i)} y^{(j)} k\left(\mathbf{x}^{(i)}, \mathbf{x}^{(j)}\right)-\sum_{i=1}^{K} \alpha^{(i)}\right)
$$

subject to constraints,

$$
\begin{gathered}
\sum_{i=1}^{K} \alpha^{(i)} y^{(i)}=0 \\
0 \leq \alpha^{(i)} \leq C
\end{gathered}
$$

Eq. (5.3) constrains the $\alpha$ 's to be non-negative and less than some constant $C$. $C$ allows for soft-margins, meaning that some of the examples may fall between the margins. This helps to prevent over-fitting the training data and allows for better generalization. 
Given $\alpha$ found in eq. (5.1), we have the following decision function:

$$
f(\mathbf{x})=\operatorname{sign}\left(\sum_{i=1}^{K} \alpha^{(i)} y^{(i)} k\left(\mathbf{x}^{(i)}, \mathbf{x}\right)\right)
$$

where the function sign returns +1 if the summation term is positive, and -1 otherwise.

If there exist vectorial representations $\overrightarrow{\mathbf{x}}^{(i)}$ for each sample $\mathbf{x}^{(i)}$ in the training set, then a vector $\overrightarrow{\mathbf{w}}$ (called weight vector) could be deduced such that,

$$
\overrightarrow{\mathbf{w}}=\sum_{i=1}^{K} \alpha^{(i)} y^{(i)} \overrightarrow{\mathbf{x}}^{(i)}
$$

and eq. (5.4) could be written equivalently as,

$$
f(\mathbf{x})=\operatorname{sign}(\langle\overrightarrow{\mathbf{w}}, \overrightarrow{\mathbf{x}}\rangle)=\operatorname{sign}\left(\sum_{\mathfrak{f}=1}^{|\mathbf{x}|} w^{\mathfrak{f}} x^{\mathfrak{f}}\right)
$$

It is noted that, in eq. (5.6), individual component of the weight vector $w^{f}$ denotes the weight (or relative importance) of feature $\mathfrak{f}$ and $x^{\mathfrak{f}}$ denotes the frequency of occurrence of $\mathfrak{f}$ in $\mathbf{x}$. Alternatively, $f(\mathbf{x})$ could be formulated as an optimization problem over $\mathbf{w}$ and solved as follows:

$$
\min _{\overrightarrow{\mathbf{w}}}\|\overrightarrow{\mathbf{w}}\|^{2}+\sum_{i=1}^{K} \max \left(0,1-y^{(i)} f\left(\overrightarrow{\mathbf{x}}^{(i)}\right)\right)
$$

MKL. With MKL, we are interested in finding $\beta$, in addition to the standard $\alpha$ of SVMs, such that

$$
k_{\text {comb }}\left(\mathbf{x}^{(i)}, \mathbf{x}^{(j)}\right)=\sum_{v \in V} \beta_{v} k_{v}\left(\mathbf{x}^{(i)}, \mathbf{x}^{(j)}\right)
$$

is a convex linear combination of all the kernels $v \in V$ with $\beta_{v} \geq 0$, where each kernel, $k_{v}$, uses a distinct set of features emanating from different views of apps [91]. The general outline of the algorithm is to first combine the kernels with $\beta_{v}=1 /|V|$, find $\alpha$, and then iteratively keep optimizing for $\beta$ and $\alpha$ until convergence. $\beta_{v}$ is also referred as the weight 
of kernel $v$, which quantifies the relative importance of view $v \in V$.

SMO-MKL. To solve for kernel weights $(\beta)$, and support vectors $(\alpha)$, simultaneously, we use the SMO based MKL algorithm proposed in [92]. For details on SMO optimization and subsequent computations of $\alpha$ and $\beta$, we refer the reader to the original work by Vishwanathan et. al. [92].

This method of MKL using the SMO algorithm is reported to be very efficient. Solving for $\beta$ and $\alpha$ with as many as 50,000 samples and 100,000 kernels has been shown to take just over 30 minutes on many applications from different domains such as Computer Vision and Bioinformatics [92]. In the MKL context, eq. (5.4) used to predict the label of a given sample is realized as follows:

$$
f(\mathbf{x})=\operatorname{sign}\left(\sum_{i=1}^{K} \alpha^{(i)} y^{(i)} k_{\text {comb }}\left(\mathbf{x}^{(i)}, \mathbf{x}\right)\right)
$$

Finding the kernel weights and support vectors across all perspectives culminates the training process, yielding an MKL-SVM ready to perform multi-view malware detection.

\subsubsection{Malicious Code Localization}

Once the MKL-SVM is trained, we use it to predict the labels of test-set apps. Subsequent to predicting an app $\mathbf{x}$ to be malicious, MKLDRoID performs the following:

- Awards an m-score to every node in x's CICFG. This helps to locate basic blocks that perform malice operations. We choose to locate malice nodes from CICFG as all the five PRGs used in MKLDRoID are its abstractions and hence all contextual subgraph features emerging from individual views could be traced back to CICFG, only.

- The $\mathrm{m}$-scores of all the methods and classes in $\mathbf{x}$ are deduced by aggregating the $\mathrm{m}$ scores of their constituent basic blocks. This helps to locate larger portions of malice codes capable enough to explain $\mathbf{x}$ 's attacks. 
Our procedure to award m-scores requires interpreting the predictions of MKL-SVM. Before delving into the details of m-score computation algorithm, we introduce some preliminaries required for this interpretation.

Interpretability: primal vs. dual formulations. The formulation of SVM discussed in eqs. (5.2) to (5.4) is known as 'dual formulation' and one mentioned in eq. (5.6) and (5.7) is known 'primal formulation'. In general, SVMs built in the former formulation are uninterpretable. On the contrary, interpreting the predictions in primal formulation is a well-studied problem as discussed in $[25,32,88]$. These methods enable us to determine the contribution of each feature to the final class prediction as described below.

Based on eq. (5.6), for a given sample $\mathbf{x}$, during the prediction of its label, $f(\mathbf{x})$, the contributions of individual features towards placing the sample on the positive (or negative) side of the decision boundary are identified by performing a point-wise multiplication of the weight and sample's vectors, i.e., the contribution of feature $\mathfrak{f}$ is deduced as:

$$
c^{\dagger}=w^{\dagger} \cdot x^{\dagger}
$$

where $x^{\uparrow}$ denotes the frequency of occurrence of feature $\mathfrak{f}$ in $\mathbf{x}$ and $w^{\mathfrak{f}}$ is its relative importance. Meaning, high frequency of features with high positive (or negative) weight would result in large positive (or negative) value of $c^{\dagger}$, pushing sample $\mathbf{x}$ significantly towards the positive (or negative) side of the decision boundary.

In the malware detection case, features with large positive and negative values of $c^{\dagger}$ characterize strong malice and benign behaviors, respectively. This interpretablity procedure could be adopted to locate contextual subgraph features from our PRGs that contribute significantly to placing a sample on the positive (i.e., malicious) side of the decision boundary.

Interpretability in MKL scenario. However, adopting this procedure with MKLSVMs is not straight-forward, as they are learnt strictly in the dual formulation. More 
specifically, we need the following in the MKL scenario to offer interpretations:

- An explicit and composite representation of a sample $\mathbf{x}$ with features from all the base kernels.

- A weight vector which quantifies the weights of features from all the base kernels.

These two vectors are not obtainable with SMO-MKL. Hence, we obtain the primal version of our MKL-SVM and use it for interpreting its predictions and computing the $\mathrm{m}$-scores as explained in algorithm 3.

Algorithm: PredictAndInterpret. The algorithm takes as inputs the training samples $\left(x_{\text {train }}\right)$ along with their labels $\left(y_{\text {train }}\right)$, the support vectors $(\alpha)$ and base kernel weights $(\beta)$ learnt in the dual formulation, test samples $\left(x_{\text {test }}\right)$ and their CIFCGs $\left(C I C F G_{\text {test }}\right)$ for which m-scores have be computed.

Firstly, The predicted labels of test samples $\left(\hat{y}_{\text {test }}\right)$ and their m-scored CICFGs (mscores $\left._{\text {test }}\right)$ are initialized to empty sets (line 2).

Then the composite representation of every training sample $\overrightarrow{\mathbf{X}}^{(i)}$ is computed in lines 3 and 4 . Here, $\oplus$ denotes concatenation operation and $\overrightarrow{\mathbf{X}}$ is obtained by concatenating the feature vectors from individual base kernels after scaling them with corresponding base kernel weights. It could be seen that,

$$
\left\langle\overrightarrow{\mathbf{X}}^{(i)}, \overrightarrow{\mathbf{X}}^{(j)}\right\rangle=\sum_{v \in V} \beta_{v}\left\langle\overrightarrow{\mathbf{x}}_{v}^{(i)}, \overrightarrow{\mathbf{x}}_{v}^{(j)}\right\rangle=\sum_{v \in V} \beta_{v} k_{v}\left(\mathbf{x}^{(i)}, \mathbf{x}^{(j)}\right)=k_{c o m b}\left(\mathbf{x}^{(i)}, \mathbf{x}^{(j)}\right)
$$

Thus, the composite representations encompass features from all views incorporating their relative importances. Subsequently, the composite weight vector, $\overrightarrow{\mathbf{W}}$, required for predictions and interpretations is obtained from representations of support vector instances in line 5. Clearly, in this vector, $\mathbf{w}^{\mathfrak{f}}$, the weight of the base kernel feature $\mathfrak{f}$ accounts for both the kernel's and feature's relative importance.

Once the weight vector of the MKL-SVM is obtained, we predict the label of every 


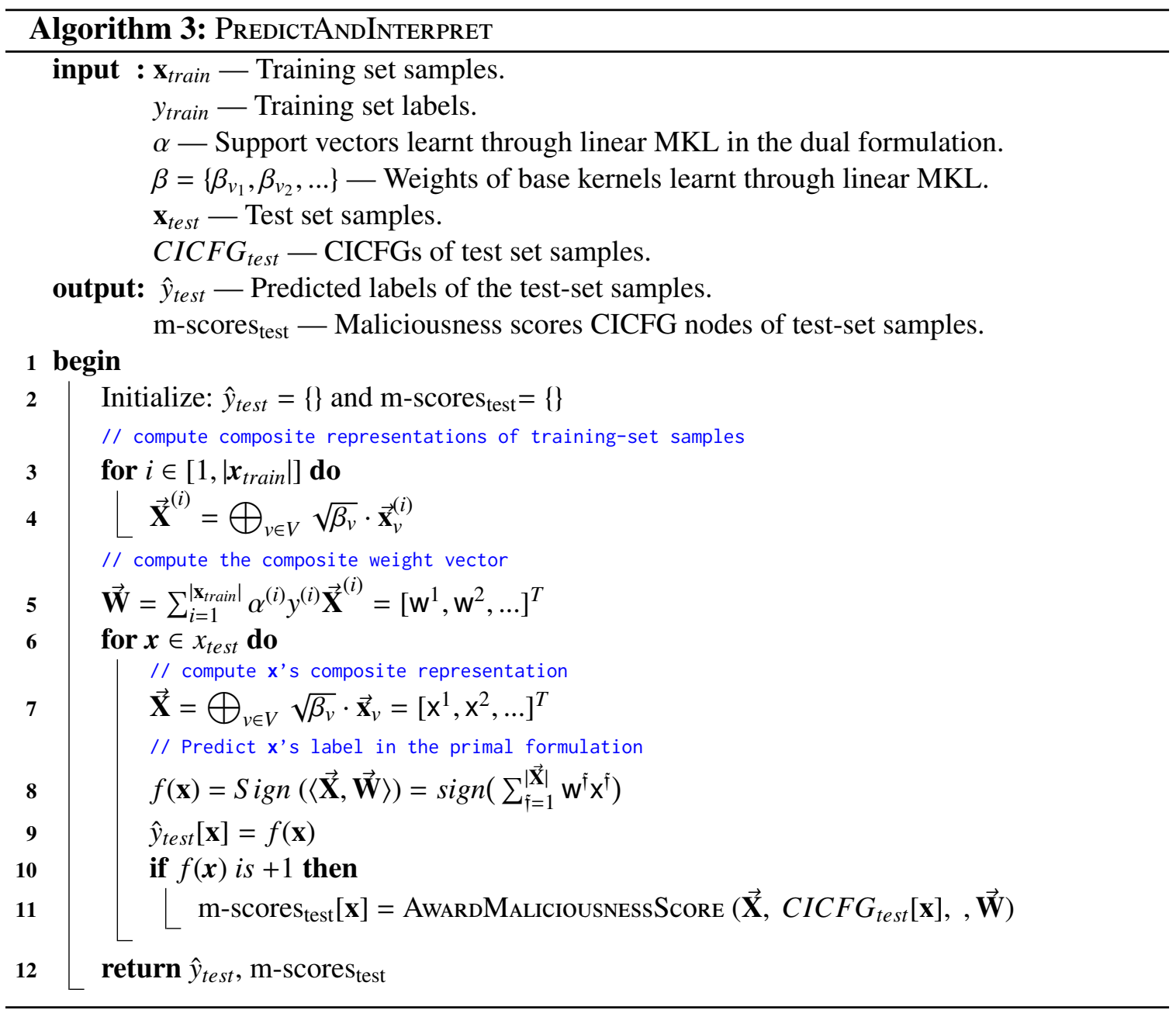

test-set sample using the primal formulation ${ }^{8}$ in line 8 . If a sample, $\mathbf{x}$, is predicted to be malware (i.e., $f(\mathbf{x})$ is +1 ), we compute the $\mathrm{m}$-scores of all nodes in its CICFG in line 11. The detailed procedure for m-score computation is explained separately in algorithm 4. Once all the test-set samples are subjected to prediction and m-score computation the results are returned in line 12 .

Algorithm: AwardMaliciousnessScore. Given the CICFG of a sample $\mathbf{x}$ that is predicted to be a malware, the goal of this algorithm is to award m-scores to each of its nodes that quantify the severity of their malice operations. To achieve this, the algorithm needs the explicit composite representation of $\mathbf{x}$ (i.e., $\overrightarrow{\mathbf{X}}$ ) and the MKL-SVM's weight vector (i.e.,

${ }^{8}$ From eqs. (5.7), it could be noted that, the prediction made in this fashion will be equivalent to one made with a linear SVM learnt as an optimization on $\overrightarrow{\mathbf{W}}$ as follows: $\min _{\overrightarrow{\mathbf{W}}}\|\overrightarrow{\mathbf{W}}\|^{2}+\sum_{i=1}^{N} \max \left(0,1-y^{(i)} f\left(\overrightarrow{\mathbf{X}}^{(i)}\right)\right)$. 


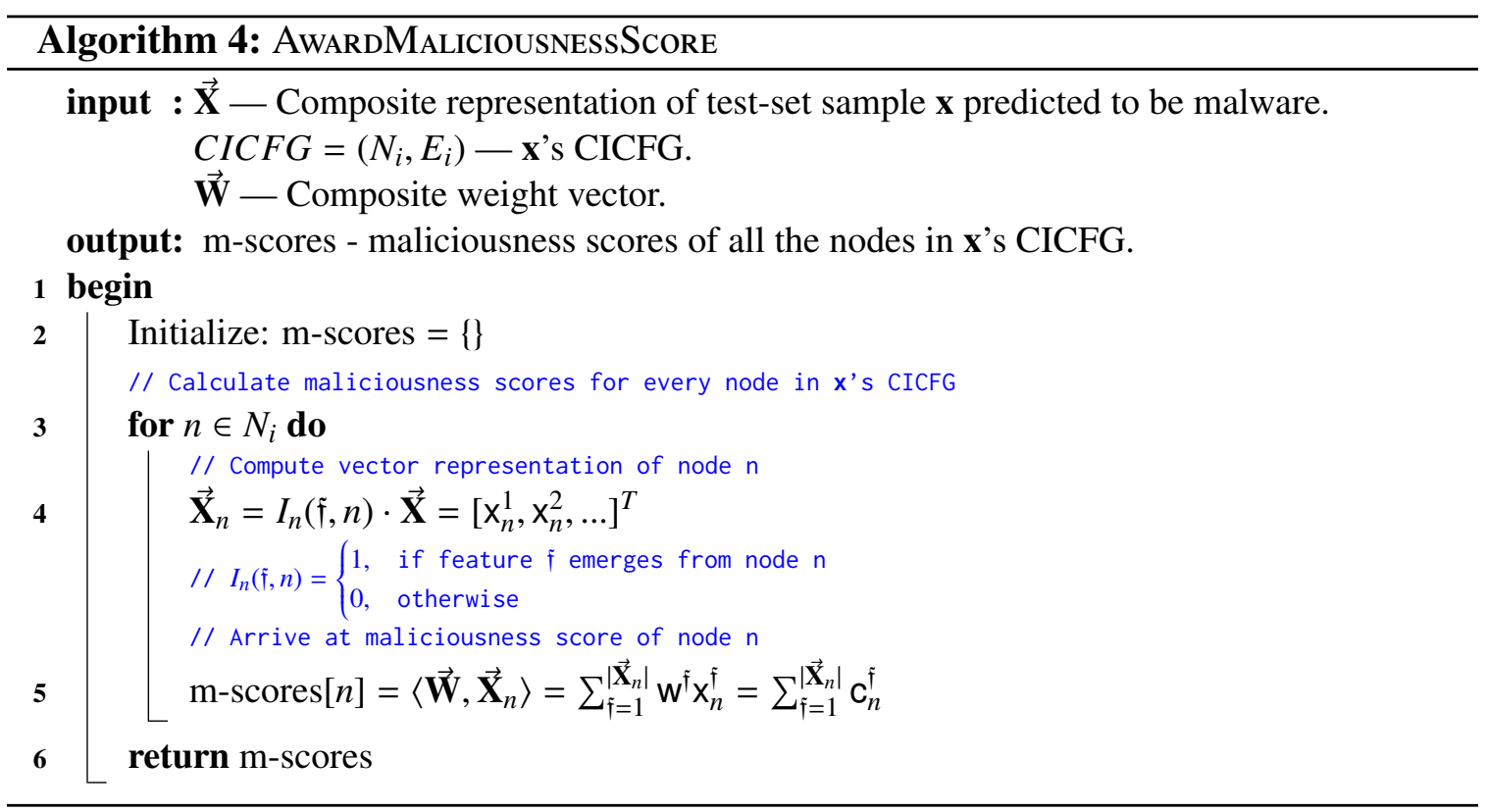

$\overrightarrow{\mathbf{W}}$ ). These values are passed as its inputs (from line 11 of algorithm 3 ).

To begin with, a dictionary of $\mathrm{m}$-scores of all the nodes are initialized (line 2). Subsequently, we loop through every node $n \in N_{i}$ in x's CICFG and compute their m-scores with a 2-step procedure in lines 4 and 5 .

In line 4 , node $n$ 's composite representation, $\overrightarrow{\mathbf{X}}_{n}$, is obtained from the sample's vector by unmasking only the contextual subgraph features that emerge from $n$. The identifier function $I_{n}$ helps this unmasking. Clearly, $\overrightarrow{\mathbf{X}}_{n}$ encompasses features from all the five views.

Once node $n$ 's representation is arrived at, we could calculate the contributions of individual features emerging from $n$ to the final prediction of $\overrightarrow{\mathbf{X}}$ using eq. (5.10). That is, the contribution of a feature $\mathfrak{f}$ from node $n$ to the final prediction $f(\mathbf{x})$ is: $\mathrm{c}_{n}^{\mathfrak{f}}=\mathbf{w}^{\mathfrak{f}} \cdot \mathbf{x}_{n}^{\mathfrak{f}}$ (where $\mathbf{x}_{n}^{\mathfrak{f}}$ denotes the frequency of occurrence of $\mathfrak{f}$ in $n$ ). Therefore, to calculate the m-score of $n$, we just need to aggregate $\mathrm{c}_{n}^{\mathfrak{f}}$ as in line 5. Finally, the m-scores of all nodes in $\mathbf{x}$ 's CICFG thus computed are returned in line 6.

Once the testing and interpretation phase finishes, we would have the predictions for all test-set samples $\left(\hat{y}_{\text {test }}\right)$ and the CICFGs of predicted malware with m-scores of their nodes $(\mathrm{m}$-scores test $)$. Subsequently, m-scores of nodes are aggregated to compute the same for 
classes and methods encompassing them as follows:

$$
\begin{aligned}
\mathrm{m}-\operatorname{score}(m) & =\sum_{n \in N_{i}} I_{m}(n, m) \cdot \mathrm{m}-\mathrm{score}(n) \\
\mathrm{m}-\operatorname{score}(c) & =\sum_{n \in N_{i}} I_{c}(n, c) \cdot \mathrm{m}-\operatorname{score}(n)
\end{aligned}
$$

where the indicator function $I_{m}$ and $I_{c}$ are defined as,

$$
\begin{aligned}
I_{m}(n, m) & = \begin{cases}1, & \text { if basic block } n \text { is contained in method } m \\
0, & \text { otherwise }\end{cases} \\
I_{c}(n, c) & = \begin{cases}1, & \text { if basic block } n \text { is contained in class } c \\
0, & \text { otherwise }\end{cases}
\end{aligned}
$$

Computing the m-scores of methods and classes culminates MKLDROID's automated detection and malicious code localization procedures. Subsequently, analysts could investigate methods and classes with high scores so as to understand malware's attacks and evasion footprints.

It could be easily seen that this process of computing m-scores could be used with base kernels to determine scores from individual views (i.e., API kernel m-scores will depend only on CADG contextual subgraphs and so on.). However, thanks to the multi-view analysis, the MKL based m-scores are more comprehensive and robust in locating malice code than those from individual views (demonstrated later through evaluations in §5.5.3).

In sum, for interpretable multi-view detection, we have trained the MKL-SVM in the dual formulation and predicted the labels of the test-set apps in the primal formulation, which helps to compute the significance of every feature towards the final prediction. To the 
best of our knowledge, there is no other work that switches MKL-SVMs formulations like ours, as this interpretablity requirement is unique to our goal of malicious code localization.

Overall, MKLDROID reaps the following advantages through its MKL:

1. MKL elegantly combines features from five different views of the app, in a way which allows the learning algorithm to take advantage of all of them simultaneously.

2. MKLDroid's learning is extendable in the sense that new perspectives (e.g., dynamic analysis or data-flows based perspectives) could be easily added to the model without complicating the final result.

3. MKLDRoID's detection process is parallelizable: computing the kernel values for testing new malware can all be done in parallel, the implication being that larger datasets can easily be handled.

4. Interpretability achieved over MKL allows MKLDROID to perform precise multi-view malicious code localization.

\subsection{Experimental Design and Implementation}

We conducted several large-scale experiments to evaluate MKLDroID's accuracy, efficiency and malicious code localization capabilities. We also perform comparative analysis with three state-of-the-art Android malware detection solutions. In this section, experimental design aspects such as RQs addressed, datasets used, evaluation setup and metrics are presented along with implementation details.

\subsubsection{Research Questions}

We intend to address the following RQs through our evaluations: 
(RQ1 Accuracy) How accurate are MKLDROID's individual perspectives in detecting malware and how does it benefit from appropriately combining them?

Accuracy under different experimental settings are investigated through the following sub-RQs:

(RQ1.1) How accurate is MKLDroID in detecting unseen malware when trained with an up-to-date dataset and how does it compare to state-of-the-art approaches?

(RQ1.2) How accurate is MKLDRoID in detecting unseen malware when trained with a dataset that is historically anterior to the evaluation set?

(RQ1.3) How accurate is MKLDROID in detecting recent malware apps collected in-thewild?

(RQ1.4) Which views of MKLDroID are most (and least) effective and does combining them through MKL offer significant improvements?

(RQ2 Efficiency) How efficient are MKLDRoID's individual views in terms of overall training and prediction time and does combining them incur significant overhead?

(RQ3 Locating malice) How accurately does MKLDRoID locate malice code in a given sample and does it explain the malicious behavior exhibited by the sample?

\subsubsection{Datasets \& Experiments}

We conducted experiments with both benchmark datasets and apps collected in-the-wild. These datasets with details such as number of samples and time of compilation are presented in Table 5.1. A total of 60,561 apps have been used in our evaluations. The design of all our experiments are summarized in Table 5.2.

\section{Controlled Experiments}

Controlled experiments were conducted on malware samples from well-known benchmark datasets and benign apps from Google Play. Three controlled experiments (CE1, CE2 and 
Table 5.1: Datasets used in evaluation

\begin{tabular}{|c|c|c|c|}
\hline $\begin{array}{c}\text { Dataset } \\
\text { Category }\end{array}$ & Dataset Name & \# of samples & $\begin{array}{c}\text { Time of } \\
\text { compilation }\end{array}$ \\
\hline \hline Malware & Drebin (DR) [25] & 5,560 & Aug'10 - Oct'12 \\
Datasets & Virus-share (VS) [14] & 24,317 & May'13 - Mar'14 \\
\hline Benign & Google Play 1 (GP1) [4] & 5,000 & Jul'12 - May'14 \\
Datasets & Google Play 2 (GP2) [4] & 10,000 & \\
& AndroidDrawer (AD) [13] & 2,399 & \\
In-the-wild & AnZhi (AZ) [6] & 3,027 & \multirow{2}{*}{ Aug'13 - Sep'16 } \\
Datasets & AppsApk (AA) [12] & 2,481 & \\
& FDroid (FD) [10] & 1,007 & \\
\hline Malice code & SlideMe (SM) [8] & 5,770 & \multirow{2}{*}{ Dec'15 } \\
Dataset & Mystique (MYST) [96] & 3,000 & \\
\hline
\end{tabular}

CE3) were conducted as described below.

CE1: 5,560 malware apps from DR and 5,000 benign apps from GP1 collections were used to form the dataset for experiment CE1. The model is trained using $70 \%$ of these samples chosen at random and is tested for accuracy on the remaining $30 \%$ samples.

CE2: 24,317 malware apps from VS and 10,000 benign apps from GP2 collections were used to form the dataset for experiment CE2. The training and test set apps ratio is same as CE1.

CE3: It could be observed that the process followed in CE1 and CE2 (i.e., splitting the malware and benign samples randomly into training and test sets and performing evaluation) is followed in almost all the previous malware detection methods such as [25, 28, 32, 58]. However, this type of evaluation has two issues:

(1) Malware in benchmark datasets were collected at a particular point in time and hence are homogeneous in terms of their attack vectors. However, malware continue to evolve and more sophisticated variants are produced subsequent to publishing such datasets [26, 33]. (2) As observed by Allix et al. [27], in these experiments, samples in the training set may be historically posterior to those in the test set. While, in the real-world/AV industry settings, when a new unseen app must be processed for detection, the training sets used are, neces- 
Table 5.2: List of experiments conducted and the composition of training and test sets

\begin{tabular}{|c|c|c|c|c|c|}
\hline $\begin{array}{c}\text { Experiment } \\
\text { type }\end{array}$ & $\begin{array}{c}\text { Experiment } \\
\# \\
\end{array}$ & $\begin{array}{l}\text { Malware } \\
\text { Samples }\end{array}$ & $\begin{array}{c}\text { Benign } \\
\text { Samples }\end{array}$ & Training set & Test set \\
\hline \multirow{3}{*}{$\begin{array}{l}\text { Controlled } \\
\text { Experiment }\end{array}$} & CE1 & DR & GP1 & $\begin{array}{l}70 \% \text { of samples (chosen at } \\
\text { random) }\end{array}$ & $30 \%$ of samples (remaining) \\
\hline & CE2 & VS & GP2 & $\begin{array}{l}70 \% \text { of samples (chosen at } \\
\text { random) }\end{array}$ & $30 \%$ of samples (remaining) \\
\hline & CE3 & $\mathrm{DR}, \mathrm{VS}$ & GP1, GP2 & $\begin{array}{l}\text { Malware: Drebin } \\
\text { Benign: GP1 }\end{array}$ & $\begin{array}{l}\text { Malware: VS } \\
\text { Benign: GP2 }\end{array}$ \\
\hline Wild Experiment & WEX & DR, VS & GP1, GP2 & $\begin{array}{l}\text { Malware: DR + VS } \\
\text { Benign: GP1 + GP2 }\end{array}$ & $\begin{array}{c}\text { Third-party market apps } \\
(\mathrm{AD}+\mathrm{AZ}+\mathrm{AA}+\mathrm{FD}+\mathrm{SM})\end{array}$ \\
\hline Malicious code localization & MCLEX & MYST & GP1 & $\begin{array}{l}66.66 \% \text { of samples (chosen at } \\
\text { random) }\end{array}$ & $\begin{array}{l}33.33 \% \text { of samples } \\
\text { (remaining) }\end{array}$ \\
\hline
\end{tabular}


sarily, historically anterior to the new app. This constraint is not considered in experiments similar to CE1 and CE2.

We address these two issue, in experiment CE3, by enforcing that the training set used for building the classifier is historically anterior to the test set. We achieve this by using the samples from DR dataset which were collected from 2010 to 2012 to train the detection model (along with GP1 benign apps) and we use the samples from VS dataset which were collected from 2013 to 2014 to test the classifier (along with GP2 benign apps).

\section{Wild Experiments}

The controlled experiments CE1, CE2 and CE3 were conducted on malware from benchmark datasets. A common observation is that real-world malware, due to their rapid evolution are more challenging to detect that the ones in the benchmark datasets [96]. A technique's effectiveness in detecting malware in the wild could not be determined through testing on such outdated homogeneous datasets. To address this, we also test our model on a large collection of recent apps from popular third-party markets (in experiment WEx). To this end, a total of 14,684 apps from five different third-party markets were collected from Aug. 2013 to Sep. 2016. To test the model on these apps, we need the ground truth labels of these apps (i.e., whether they are malicious or benign). To this end, following the software security research practices proposed in [25] and [26], we leveraged on the VirusTotal web portal $^{9}$ to infer their ground truth labels. Out of these apps, 6,128 are found to be malware. Thus, in WEx, all the malware apps from DR and VS (29,877 in total) and benign apps from GP1 and GP2 (15,000 in total) collections were used to train the classifier. The test set comprises of 14,684 wild apps. It is noted that this wild dataset contains samples that were released over a larger period of time than the one used for evaluating CASANDRA (see §4.4.2).

\footnotetext{
${ }^{9}$ https://www.virustotal.com
} 


\section{Malicious Code Localization Experiment}

In this experiment, we intend to evaluate MKLDroID's capabilities to locate the malicious code in a given sample. More specifically, we explore whether it could locate malice methods or classes involved in the sample's attacks.

Quantitative Evaluation. As mentioned earlier, though a vast body of literature on Android malware detection approaches exists, none of them systematically addressed the problem of locating malice code in given sample. This is partly due to the fact that none of existing datasets (incl. DR [25], VS [70], AndroZoo [69]) provide ground-truth on the location of malice code ${ }^{10}$ such as names of methods/classes involving in malice operations. They just provide labels to ascertain whether a sample holistically is benign or malicious. Obviously, with these datasets, one could not quantitatively evaluate malicious code localization capabilities. In order to address this, we extended an existing dataset as follows. Recently, Mystique [96] proposed an Evolutionary Computation based method to automatically generate new malware samples learning from attack and evasion strategies of benchmark malware. Mystique provided a dataset of 10,000 such automatically generated malware with the names of the classes that contain malice code. However, almost all the code in these samples are either malicious or from commonly used libraries (e.g., android.support) and there are no benign functionalities. In other words, none of these apps are repackaged malware and hence do not cater well to the real-world needs of locating malice code in repackaged malware. Hence to extend it by randomly choosing 3,000 apps from this dataset and piggybacked the same on benign apps from Google Play. Hence, for each of these apps we are certain of the following: (i) they contain both benign and malice code, (ii) names of the classes that contain malice code. We refer to this dataset as MYST

${ }^{10} \mathrm{Recently,} \mathrm{Li} \mathrm{et} \mathrm{al.} \mathrm{[70]} \mathrm{provided} \mathrm{a} \mathrm{dataset} \mathrm{of} \mathrm{repackaged} \mathrm{apps} \mathrm{of} \mathrm{the} \mathrm{form:} \mathrm{(app1,} \mathrm{app2),} \mathrm{where} \mathrm{app} 1$ is the original (benign) app and (app2) is the repackaged version of app1. However, they do not ascertain whether or not the new code injected in app2 is malicious. In fact, exploring this dataset, we observe that a majority of the repackaged apps were adware or other type of PHAs. Hence, we refrain from using this dataset which lacks precise ground truth labels on malice methods and classes in our experiments. 
and use the same in experiment MCLEx, where we investigate MKLDroID's malicious code localization capabilities quantitatively, as follows.

We train our model using 2,000 malware from MYST dataset and 2,000 benign apps (that were not used for piggybacking). Subsequently, we test the model on the remaining 1,000 MYST samples. Remember, during testing, MKLDroID assigns m-scores to every class in an app. Hence, we investigate whether the classes with highest m-scores are indeed malicious using MYST's ground-truth.

Qualitative Evaluation. To perform qualitative evaluation we do not need manual annotations on classes/methods containing malice code. All we need are samples that contain malice code at least in one of their classes/methods and we could use MKLDRoID on them to investigate if it locates such code. Hence, for qualitative evaluation, we choose the DR dataset ${ }^{11}$ that contains real-world malware with malice code spread across many methods and classes. The experimental settings in CE1 are reused in this evaluation. We manually whether investigate classes and methods with high m-scores correspond to the sample's attacks.

\subsubsection{Experimental Setup}

All the experiments were conducted on a server with 20 cores of Intel(R) Xeon(R) CPU E5-2699 v3 @ 2.30GHz and 200 GB RAM running Ubuntu 14.04.

\subsubsection{Implementation and Comparative Analysis}

MKLDroID is implemented in approximately 9,700 lines of Python, Java and C++ code. Androguard [16] and Soot [17] have been used to build the PRGs and infer the reachability contexts of PRG nodes. For SMO-MKL functionalities, the source code provided by SVN Vishwanathan et al. [92] has been used.

\footnotetext{
${ }^{11}$ More than $80 \%$ of samples in this dataset are piggybacked malware thus making this dataset amenable
} for our qualitative analysis [70]. 
Comparison with state-of-the-art solutions. Similar to chapter 3, our approach is compared against three state-of-the-art ML based Android malware detection solutions, namely, DreBin [25], CSBD [26] and Adagio [32].

\subsubsection{Evaluation Metrics}

Standard evaluation metrics such as Precision, Recall and F-measure are used to determine the effectiveness of malware detection. All these values are in the range $[0,1]$. Higher values indicate accurate detection. Efficiency is determined in terms of training and testing durations (in seconds). Lower training and testing durations indicate scalable detection. For evaluating malicious code localization, False Positive Rate (FPR) and False Negative Rate (FNR) measures are used. These are expressed as percentage values. Lower FPR and FNR indicate precision and completeness in detection, respectively.

\subsection{Results and Discussions}

The evaluation, results and relevant discussions pertaining to each of the RQs is presented in this section. The accuracy and efficiency results for controlled and in-the-wild experiments is presented in subsections $§ 5.5 .1$ and $§ 5.5 .2$, respectively. For malicious code localization, a qualitative evaluation which involves case studies on two well-known malware families and a quantitative evaluation on the homegrown dataset are presented in §5.5.3.

\subsubsection{RQ1: Accuracy}

\section{RQ1.1 Accuracy on benchmark datasets}

As mentioned earlier, in experiments CE1 and CE2, $70 \%$ of samples were randomly chosen from the evaluation datasets and used for training the classifier and the remaining $30 \%$ samples are used to test its performance. The hyper-parameters of classifier are determined 
Table 5.3: CE1 Results: Precision, Recall \& F-measure (avg \pm std.)

\begin{tabular}{|l|l|l|l|}
\hline Technique & $\mathrm{P}$ & $\mathrm{R}$ & $\mathrm{F}$ \\
\hline \hline API kernel & $0.974( \pm 0.004)$ & $0.989( \pm 0.001)$ & $0.982( \pm 0.004)$ \\
Permission kernel & $0.960( \pm 0.010)$ & $0.978( \pm 0.008)$ & $0.970( \pm 0.011)$ \\
Src-sink kernel & $0.802( \pm 0.047)$ & $0.734( \pm 0.012)$ & $0.766( \pm 0.008)$ \\
Instruction kernel & $0.967( \pm 0.005)$ & $0.989( \pm 0.004)$ & $0.979( \pm 0.004)$ \\
Signature kernel & $0.971( \pm 0.005)$ & $\mathbf{0 . 9 9 0}( \pm 0.008)$ & $0.981( \pm 0.005)$ \\
\hline Uniform kernel & $0.987( \pm 0.003)$ & $0.982( \pm 0.002)$ & $\mathbf{0 . 9 8 5}( \pm 0.003)$ \\
\hline MKLDROID & $\mathbf{0 . 9 8 8}( \pm 0.004)$ & $0.982( \pm 0.004)$ & $\mathbf{0 . 9 8 5}( \pm 0.003)$ \\
\hline Drebin [25] & $0.980( \pm 0.002)$ & $\mathbf{0 . 9 9 0}( \pm 0.001)$ & $\mathbf{0 . 9 8 5}( \pm 0.002)$ \\
\hline Adagio [32] & 0.960 & 0.974 & 0.967 \\
\hline CSBD [26] & $0.954( \pm 0.005)$ & $0.987( \pm 0.008)$ & $0.970( \pm 0.005)$ \\
\hline
\end{tabular}

Table 5.4: CE2 Results: Precision, Recall \& F-measure (avg \pm std.)

\begin{tabular}{|l|l|l|l|}
\hline Technique & $\mathrm{P}$ & $\mathrm{R}$ & $\mathrm{F}$ \\
\hline \hline API kernel & $\mathbf{0 . 9 6 6}( \pm 0.010)$ & $0.943( \pm 0.016)$ & $0.954( \pm 0.024)$ \\
Permission kernel & $0.962( \pm 0.022)$ & $0.931( \pm 0.038)$ & $0.946( \pm 0.017)$ \\
Src-sink kernel & $0.710( \pm 0.035)$ & $0.728( \pm 0.052)$ & $0.719( \pm 0.044)$ \\
Instruction kernel & $0.954( \pm 0.010)$ & $0.946( \pm 0.018)$ & $0.950( \pm 0.010)$ \\
Signature kernel & $0.951( \pm 0.014)$ & $0.944( \pm 0.018)$ & $0.948( \pm 0.011)$ \\
\hline Uniform kernel & $0.964( \pm 0.013)$ & $0.952( \pm 0.009)$ & $0.958( \pm 0.012)$ \\
\hline MKLDROID & $\mathbf{0 . 9 6 6}( \pm 0.015)$ & $\mathbf{0 . 9 8 0}( \pm 0.008)$ & $\mathbf{0 . 9 7 3} \pm 0.009)$ \\
\hline Drebin [25] & $0.960( \pm 0.007)$ & $\mathbf{0 . 9 8 0}( \pm 0.009)$ & $0.971( \pm 0.006)$ \\
\hline Adagio [32] & 0.960 & 0.960 & 0.960 \\
\hline CSBD [26] & $0.914( \pm 0.028)$ & $0.958( \pm 0.008)$ & $0.938( \pm 0.015)$ \\
\hline
\end{tabular}

on the training set (using 5-fold cross-validation), whereas the test set is only used for determining the final detection performance. We repeat this procedure five times and average the results. In order to study the effectiveness of individual perspectives, we report the prediction results using individual base kernels and the uniform kernel (which is the mean of all base kernels).

The results for experiments CE1 and CE2 along with comparison to state-of-the-art techniques is presented in tables 5.3 and 5.4, respectively. The following inferences are drawn from these tables:

- At the outset, we observe that all individual perspectives have certain effectiveness in detecting malware. This is reflected by the fact that all the base kernels get more than 
$75 \%$ F-measure in both the experiments. In fact, 4 out of 5 base kernels offer comparable F-measures to state-of-the-art approaches.

- Out of the base kernels, API kernel achieves the best performance in both the experiments. Meaning, context-aware structural API dependencies turn out to be excellent features for detecting malware. In fact, this observation goes hand-in-hand with the fact that API related features (API frequencies and ngrams [25, 41], API sequences [37], subgraphs $[28,31,38]$, dependencies [58,59], etc.) are the most popular features in Android malware detection literature.

- The performances of instruction and signature kernels are very close in both the experiments and are marginally less effective than those of API kernel. This is because both these kernels capture structural dependencies from the apps that are sparse, obfuscation resilient and abstract.

- Permission kernel achieves significantly lesser standalone F-measure than the three above mentioned kernels. Understandably, permissions are more coarse-grained features compared to API and instruction sequences. This leads to many misclassifications thereby hampering the kernel's accuracy.

- Src-sink kernel obtains the least F-measure indicating that it exhibits least malware detection potential. Note that CSSDG actually captures control flows across source and sink nodes and this does not necessarily mean there are data-flows (i.e., information leak) in the suspected control flow paths ${ }^{12}$. We conjecture that considering control-flow paths as proxies for detecting information leak attacks leads to lots of false positives and thus poor detection.

- The main justifications for using MKL to integrate the perspectives could be observed by comparing the uniform kernel and MKLDRoID's performances. The uniform kernel ${ }^{12}$ Remember, we intend to avoid computing expensive data-flows in the app and believe other perspectives (computed at much lesser expense) would complement and mitigate the absence of data-flow related features. 
which assigns equal weights to all the base kernels performs on par with MKLDRoID obtaining best F-measure in CE1. However, in CE2, which involves larger datasets with more families of malware, the former fails to outperform the latter. Leveraging on MKL, our approach identifies the most appropriate linear combination of the base kernels and obtains better precision, recall and F-measure. This justifies the need for having a nonuniform combination of the base kernels.

- Comparing against state-of-the-art approaches, we observe that MKLDRoID achieves performance on par with the best performing technique i.e., Drebin, while outperforming CSBD and AdAGio in CE1. It is important to note that Drebin's features are perfectly engineered to offer excellent performance on the DR dataset. Similar observations have been reported through other large-scale studies such as [37, 68]. Hence achieving onpar performance convincingly reveals the detection potentials of MKLDROID. Notably, all the approaches exhibit lesser F-measure in CE2 than in CE1, revealing that the former setting is more challenging. Interestingly, MKLDROID achieves best results in CE2 outperforming all the state-of-the-art approaches and the uniform kernel.

Having sufficiently established the detection capabilities of base kernels and the need for MKL, we exclude evaluations and discussions on individual base kernels in forthcoming sub-RQ 1.2 and 1.3 .

\section{RQ1.2 History-aware training and evaluation}

We now report the results on experiment CE3, where we enforced the constraint: the training set is historically anterior to the test set in Table 5.5. Before discussing the results of individual techniques, we present an important inference from Table 5.5: the performances of all the state-of-the-art methods (and MKLDroID) are significantly worse when the test set is posterior to the training set. This is in line with the observations reported in [27] and [33]. The reason for this drop in performance and relevant observations are reported 
Table 5.5: CE3 Results: Precision, Recall and F-measure

\begin{tabular}{|l|c|c|c|}
\hline Technique & $\mathrm{P}$ & $\mathrm{R}$ & $\mathrm{F} 1$ \\
\hline \hline MKLDroid & $\mathbf{0 . 9 9}$ & $\mathbf{0 . 5 5}$ & $\mathbf{0 . 7 1}$ \\
DrEBIN [25] & $\mathbf{0 . 9 9}$ & 0.36 & 0.52 \\
ADAGIo [32] & 0.98 & 0.41 & 0.58 \\
CSBD [26] & 0.98 & 0.43 & 0.60 \\
\hline
\end{tabular}

below.

Unlike experiments CE1 and CE2, in CE3, the test set apps are almost one year historically posterior to the training set ones. Some of these malware could belong to a new family that emerged after the training set was compiled or could be a more sophisticated variant of a family that surfaced in the training set. Hence, all the knowledge acquired and used by the classifier would not be sufficient or relevant.

In this aspect, CE3 models the real-world detection settings more closely. The practical potentials of a technique will be revealed in this experiment. Only techniques that capture most aspects of an app that distinguishes malicious behavior from benign ones could perform well. Techniques that overfit the training set will perform poorly particularly in CE3.

From Table 5.5, it is evident that MKLDRoID outperforms all the state-of-the-art techniques, significantly in CE3. The margin of improvement is $11 \%$ which is much higher compared to that of CE1 and CE2. Furthermore, the following observations are made from Table 5.5:

- Precision. Clearly the precision of all the techniques under comparison are very high and almost same (in the range $[0.98,0.99]$ ). This reveals that all the techniques could very well detect test set malware that are similar to training set malware (e.g., similar variants of families exposed during training).

- Recall. However, the real difference between MKLDroID and other techniques lies in the recall value. The other techniques have much poorer recall (well below 0.50). This 
reveals that even though they could detect known malware, they do not generalize well to find newer variants or families. MKLDroID's recall value suggests that, it detects newer variants/families better. Nevertheless, its recall is also not very high, indicating high number of false negatives. This is because, though MKLDRoID can generalize well, it cannot automatically adapt to the evolution in malware samples. More specifically, MKLDRoID is a batch-learning based approach, where we use a batch of samples to train the model. Subsequent to training, the model is used to only predict the labels of the samples that stream-in. It cannot automatically update its learning unless it is retrained with a fresher or more recent training set. As noted in the previous chapter (chapter 4), this is an inherent limitation of batch-learning based solutions. We discuss our measures to address this adaptiveness issue in the MKL setting, later in $§ 5.7$.

- Particularly, the recall and F-measure values of Drebin, which performed well in CE1 and CE2 are very poor in CE3. This indicates that the features captured by Drebin, are too much dependent on the training set and it could reliably detect a new test set malware only if it is very similar to training set malware. In other words, DreBIN suffers from overfitting. This observation is reinforced by the fact that some of Drebin's features such as URLs, names of components, etc. do not adapt to unseen test set apps well. For instance, the component names in test set apps may be vastly different from training set ones and learning these becomes useless during testing. This introduces noisy features, leading to overfitting and poor detection rates.

- Unlike Drebin, both AdAGio and CSBD use features that are not very much training setspecific. This helps them generalize well. Furthermore, CSBD uses feature selection to prune irrelevant features and this helps improving its recall and F-measure. However, these approaches perform more like signature-based detection approaches (as they learn instruction and CFG signatures) and this leads to an overall poor performance in CE3 where there is significant drift in the test set. MKLDROID outperforms these approaches 
Table 5.6: WEx Results: Precision, Recall and F-measure

\begin{tabular}{|l|c|c|c|}
\hline Technique & $\mathrm{P}$ & $\mathrm{R}$ & $\mathrm{F} 1$ \\
\hline \hline MKLDroID & $\mathbf{0 . 6 0}$ & $\mathbf{0 . 9 1}$ & $\mathbf{0 . 7 2}$ \\
DreBin [25] & 0.53 & 0.78 & 0.63 \\
ADAGIo [32] & 0.47 & 0.87 & 0.61 \\
CSBD [26] & 0.51 & 0.87 & 0.64 \\
\hline
\end{tabular}

by $11 \%$ or more F-measure.

\section{RQ1.3 Accuracy on the wild dataset}

We now report the detection results obtained on recent wild apps. In this experiment, WEx, the models are trained using all the malware from benchmark datasets and benign apps from Google Play and tested on apps collected from third-party markets. The precision, recall and F-measure values of all the approaches under comparison are presented in Table 5.6 .

Evidently, in this experiment, MKLDroID outperforms the state-of-the-art approaches significantly (i.e., by $8 \%$ or more F-measure), similar to CE3. The following observations are made from Table 5.6.

- At the outset, conspicuously, for all the approaches under study, performing detection on the wild dataset is more challenging than the benchmark ones. All the four methods produce F-measure in the range $[0.60,0.72]$ in WEx. This observation holds despite the fact that all models are trained with the largest amount of data in WEx, out of all the four experiments. This reveals that the homogeneity observed in the attack vectors of benchmark malware is not observed in the same magnitude among malware apps in the wild.

- Precision. Even though the F-measures of these approaches in WEx are similar to those of CE3, a major difference lies in the precision values. In CE3, all the approaches identified test set malware that exposed in the training set quite well, leading them to better 
Table 5.7: Weights of MKLDroID's base kernels - learnt using SMO-MKL [92]

\begin{tabular}{|c|c|c|}
\hline Kernel & CE1 & CE2 \\
& Weight (norm $=\mathrm{L} 2, \mathrm{C}=100)$ & Weight $($ norm $=\mathrm{L} 2, \mathrm{C}=1)$ \\
\hline \hline API kernel & $\mathbf{1 1 9 . 8 8}( \pm \mathbf{1 . 1 6})$ & $\mathbf{2 9 . 6 6}( \pm \mathbf{0 . 1 8})$ \\
Permission kernel & $46.41( \pm 0.73)$ & $13.37( \pm 0.12)$ \\
Src-sink kernel & $26.41( \pm 0.80)$ & $12.45( \pm 0.15)$ \\
Instruction kernel & $95.34( \pm 1.09)$ & $16.27( \pm 0.81)$ \\
Signature kernel & $95.77( \pm 1.31)$ & $14.26( \pm 0.71)$ \\
\hline
\end{tabular}

precisions (i.e., in the range $[0.98,0.99])$. However, in WEx, their precision values are in the range $[0.47,0.60]$. This on average is $45 \%$ lesser than CE3's precision values. This clearly illustrates that the false positives are significant across all these approaches, as distinguishing the behaviors of benign and malware apps in the wild is more challenging.

- Recall. In CE3, the test set malware are entirely posterior to the training set ones, leading to a larger scope for malware evolution. Consequently, all the four approaches obtained poor recall values (i.e., in the range $[0.43,0.55])$. However, in WEx, the period of collection of test samples, overlaps with certain months of the training set compilation period. This enables the detection models to operate under a less vigorous population drift setting. This directly reflects in their lesser false negative and consequently, better recall values. However, we note these precision and recall values are not as high as in CE1 and CE2, reinforcing the need for detecting and adapting to malware population drift observed in the wild.

- Vs. other approaches. Out of the state-of-the-art approaches, DREBIN and CSBD perform reasonably better than AdAGio. MKLDroid outperforms these methods by $8 \%$ or more F-measure.

\section{RQ1.4 Effectiveness of individual views}

Having sufficiently established that capturing context-aware multi-view features facilitates MKLDRoID to achieve superior accuracies, we now intend to gain insights into the contri- 
butions of individual views to its performance. In this sub-RQ, we perform a sensitivity analysis on MKLDRoID's base kernels both quantitatively and qualitatively. In particular, we study whether there is any correlation between the weights that MKL offers to individual base kernels and their detection rate, rank the perspectives based on these weights and also suggest which perspectives to use when resources and time are at a premium.

Quantitative analysis. As explained in \$5.3.4, the SMO-MKL algorithm used in MKLDRoID assigns weights to individual base kernels yielding an appropriate linear combination of the base kernels. These weights of the base kernels in CE1 and CE2 are reported in Table 5.7. The following observations are made from the table:

- A straight-forward observation is that, in our framework, the weight of a base kernel signifies its malware detection potential. Higher the weight, better is the kernel's detection potential. For instance, the API and Src-sink kernels get the most and least significant weights in both the experiments. This is in line with the fact that these kernels offered the best and the worst standalone accuracies, respectively. However, we note that there need not be direct correlation between the base kernel weights and their accuracies when non-linear kernel combinations or hyper-kernels are used. Our base kernels' weight assignments are particularly intuitive as we perform linear MKL in our framework.

- Also, the weights of Instruction and Signature kernels are very similar. This stems from the fact that these kernels capture similar structural information at similar level of granularity, exhibiting identical detection capabilities. Interestingly, Signature kernel obtains more weight than Instruction kernel in CE1, whereas the weight significances are other way around in CE2. We believe this due to the fact that the dataset used in CE1 (i.e., DR) is comparatively more homogeneous than in CE2 (i.e., VS). Homogeneous malware tend to exhibit similar CFG signature thereby helping the Signature kernel to get a trifle better detection rates.

- Permission kernel's weights rank just below the Signature and Instruction kernels, 


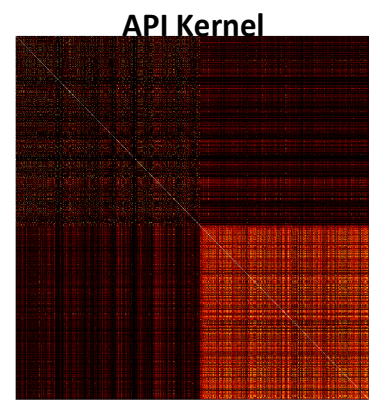

(a)

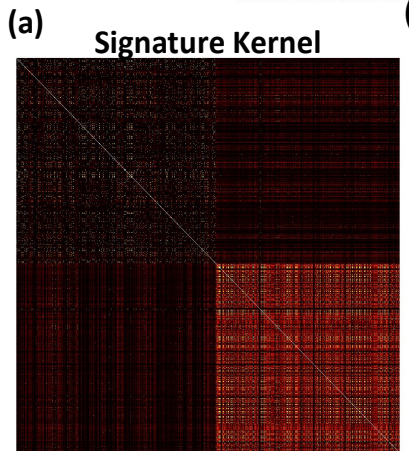

(e)

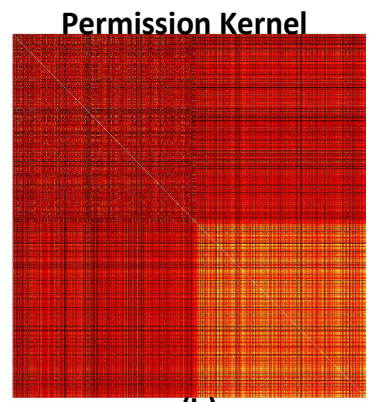

(b)

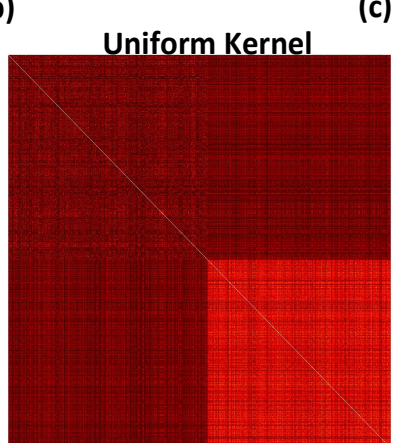

(f)

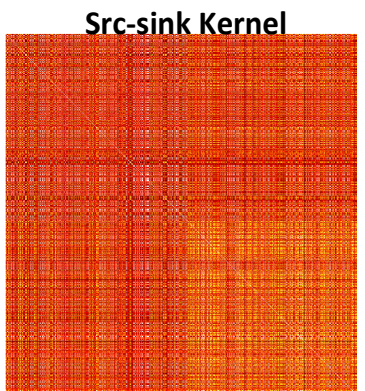

(c)

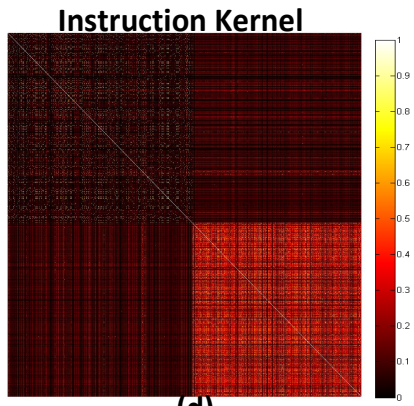

MKLDroid

(d)

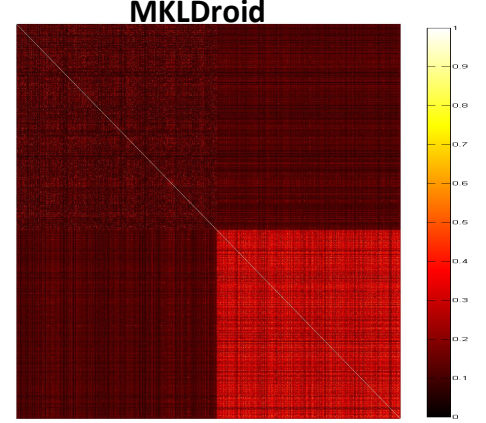

(g)

Figure 5.3: Qualitative Analysis: Comparison of base kernels and kernel combinations through visualizing kernel matrices as heatmaps

going hand-in-hand with its prediction quality.

Qualitative analysis. The detection capabilities of the base kernels and kernel combinations could also be inferred by visualizing the kernel matrices. To this end, we present the kernel matrix of all the samples used in CE1 as a heat map in Fig. 5.3. The first (top-left) 5,000 samples are the benign apps from the GP1 collection and the subsequent (bottom-right) 5,600 samples malware from DR collection. Every cell in the kernel matrix represents the similarity value between a pair of apps. Dark and light shades in cells indicate low and high similarity values, respectively.

It could be clearly seen that the malware apps exhibit high similarities among them in all the perspectives compared to the benign apps. This qualitative depiction reinforces the observations on homogeneity in DR collection that we discussed above. Also, the inferences on individual base kernel's detection potentials discussed in RQ1.1 are observed qualitatively from figures 5.3 (a) - (e). For instance, the API kernels separates the malware 
and benign samples better than other base kernels. Also, the non-uniform linear combination of kernels learnt by SMO-MKL (Fig. 5.3 (g)) offers the best separation between the samples of the two classes.

Ranking the individual perspectives. With the explanations mentioned above, it is straight-forward to rank the kernels. Considering that the base kernel weights quantifies their relative importances in the MKL setting, the following rankings are obtained: rank 1: API kernel, rank 2: shared by Signature and Instruction kernels, rank 4: Permission kernel and rank 5: Src-sink kernel. However, the scalability of these kernels differ significantly. Hence we defer discussing which subset of kernels to use when time/resources are scarce, to $\$ 5.5 .2$, where we report the base kernel efficiencies.

Summarizing the inferences from RQ1, we conclude that individual base kernels of MKLDROID exhibit certain malware detection potentials and combining them appropriately through MKL enables them to complement each other to achieve superior accuracies. This context-aware multi-view learning makes MKLDROID powerful enough to detect more sophisticated and newer variants of malware, leading it to significantly outperform state-ofthe-art methods in challenging real-world experimental settings.

\subsubsection{RQ2: Efficiency}

We now present the results for efficiency of our base kernels, MKLDroID and state-of-theart approaches in terms of average training and testing durations in experiments CE1 and CE2 (across 5 runs) in Table 5.8. The training and testing time depends on factors such as sample size, number of features and type of the kernel/learner being used. Hence, these values are also reported. It is noted that the trend in efficiency values remain same in other experiments (i.e., CE3 and WEx) as well. Owing to space constraints, they are omitted.

The following are noted as well: (i) for the base kernels that involve feature selection (Instruction \& Signature) the reported training duration includes time taken for feature 
Table 5.8: CE1 and CE2 Efficiency: Average training and testing durations

\begin{tabular}{|c|c|c|c|c|c|c|c|}
\hline \multirow{2}{*}{ Technique/Kernel } & \multirow{2}{*}{$\begin{array}{c}\text { Kernel } \\
\text { Type }\end{array}$} & \multicolumn{3}{|c|}{ CE1 } & \multicolumn{3}{|c|}{ CE2 } \\
\hline & & \# feature & $\begin{array}{c}\text { Feature selection + } \\
\text { Training duration (in sec.) }\end{array}$ & $\begin{array}{l}\text { Testing duration } \\
\text { (in sec.) }\end{array}$ & \# features & $\begin{array}{c}\text { Feature selection }+ \\
\text { Training duration(in sec.) }\end{array}$ & $\begin{array}{l}\text { Testing duration } \\
\text { (in sec.) }\end{array}$ \\
\hline API & Linear & 102633 & 0.56 & 0.0013 & 115380 & 0.63 & 0.0014 \\
\hline Permission & Linear & 253 & 0.02 & 0.0001 & 367 & 0.02 & 0.0001 \\
\hline Src-sink & Linear & 129 & 0.01 & 0.0001 & 147 & 0.02 & 0.0001 \\
\hline Instruction & Linear & 5000 & 0.36 & 0.0003 & 5000 & 0.38 & 0.0004 \\
\hline Signature & Linear & 5000 & 0.33 & 0.0002 & 5000 & 0.37 & 0.0004 \\
\hline Uniform kernel & $\begin{array}{l}\text { Linear combination } \\
\text { of base kernels }\end{array}$ & 113015 & 0.73 & 0.0016 & 125894 & 0.81 & 0.0016 \\
\hline MKLDROID & $\begin{array}{l}\text { Linear combination } \\
\text { of base kernels }\end{array}$ & 113015 & 4.67 & 0.0725 & 125894 & 5.30 & 0.0870 \\
\hline DREBIN & Linear & 170185 & 0.75 & 0.0017 & 390431 & 0.98 & 0.0020 \\
\hline CSBD & N/A & 5000 & 13.62 & 1.24 & 5000 & 46.89 & 1.34 \\
\hline ADAGIO & HI kernel & 32768 & 2783 & 792 & 32768 & 5188 & 961 \\
\hline
\end{tabular}


selection, corresponding dimensionality reduction and training the models, and (ii) in the case of MKLDRoID, testing duration includes time taken for switching from dual to primal formulation and then predicting the labels (see §5.3.5). Expectedly, CE2's training and testing durations are longer than CE1 as it involves larger datasets. From Table 5.8 the following inferences are drawn:

- Since PRG vectors from individual views (e.g., CADG vectors for API kernel, and so on) are used in conjunction with linear SVMs, their base kernel training and testing durations is very less. Since, both uniform kernel and MKL perform classification through linear combinations of base kernels, they require slightly more training and testing durations.

- Comparing individual views. Out of the base kernels, Src-sink and Permission kernels use least number of features and hence emerge as the fastest ones in terms of both training and testing durations. On the other hand, API kernel uses very large number of features and hence emerges as the slowest base kernel. In the case of Instruction and Signature kernels, the original number of features are very large i.e., more than 300,000 in both CE1 and CE2. Hence, we reduce this dimensionality using feature selection. This results in significantly larger training and testing durations. In summary, in terms of efficiency, MKLDRoID's base kernels could be ranked as follows: rank 1: Src-sink, rank 2: Permission, rank 3: is be shared by both Instruction and Signature and rank 5: API. Evidently, when we take into account accuracy results from tables 5.3 and 5.4 as well, the two most efficient views are not sufficiently accurate. Hence, when there are severe resource/time constraints, we recommend using only the Instruction and/or Signature kernels which achieve high accuracy with reasonable efficiency. Following the above-mentioned strategy, the accuracy-efficiency trade-off among the individual perspectives of MKLDRoID could be learnt from tables 5.3, 5.4 and 5.8. For particular time or resource constraints, one may decide on which subset of views to use, based on this trade-off. 
- Vs. Drebin. Drebin extracts light-weight features (i.e, no PRG based features are extracted) and uses linear SVM for classification. This helps DREBIN to achieve very high efficiency. Comparing Drebin's efficiency against those of MKLDroID's base kernels, we could see that all of them are faster than DreBIN. This is mainly because these base kernels use lesser number of features than Drebin. Uniform kernel's efficiency is comparable to that of DreBIN. However, none of the base kernels could outperform DreBIN in terms of accuracy. Meaning, base kernels achieve this efficiency at the cost of accuracy. Also, when the base kernels are integrated, MKLDRoID becomes almost 5 to 6 times slower in terms of training duration and 42 to 44 times slower in terms of testing duration than Drebin. This is mainly because of the time MKLDroID spends to learn the base kernel weights using SMO-MKL and switching to primal formulation, subsequently.

- Vs. CSBD. CSBD uses RF classifier with 100 estimators and 5000 features (selected using Information Gain values). Since RFs are quasi-linear models, they require significantly more training and testing durations than approaches that use linear models (i.e., Drebin and MKLDroID). In particular, MKLDRoId is more than 2 times faster in terms of training and more than 17 times faster in terms of testing durations than CSBD.

- Vs. Adagio. Adagio uses a kernel SVM, a computationally heavy learner, as it aims to learn non linear decision boundaries. Furthermore, it uses HI kernel with large number of features in the primal formulation, as it aims to build an interpretable model. These factors render the approach very much inefficient with practically intractable training and testing durations. In particular, MKLDROID is more than 500 times faster in terms of training and more than 10,000 times faster in terms of testing durations than AdAGIO. 
Summarizing RQ2 evaluations, we conclude that though MKLDROID uses five base kernels, it sticks to linear combinations of those kernels and hence requires modest training and testing durations. It is more efficient than two of the state-of-the-art approaches. Furthermore, the trade-off between base kernels' accuracy and efficiency is determined, which would help in selecting only a few of them when resources and time constraints are severe.

\subsubsection{RQ3: Locating Malice Code}

In this RQ, we intend to investigate whether MKLDRoID is capable of reliably locating malice code in a given sample both qualitatively and quantitatively. As stated earlier, all the existing approaches ${ }^{13}$ (incl. DreBIN and CSBD) are not capable of doing such localization. Since, fine-grained malicious code localization is MKLDRoID's unique feature, we could not compare this with any existing technique. Hence, we illustrate how MKLDRoID achieves it in this subsection and discuss how it supports human analysts to visualize PRGs from different perspectives facilitating precise understanding of malice behaviors

\section{Qualitative Evaluation}

To perform qualitative evaluation we trained the model as in experiment CE1 and it to locate malice code in the test set apps. Once a test-set app is predicted to be malicious, MKLDRoID assigns m-scores to each CICFG nodes (i.e., basic block) as described in §5.3.5. Hence, for the qualitative analysis part, we manually investigate the code in basic blocks with high m-scores to check whether they indeed are a part of the malware's attack vector.

The above mentioned analysis yielded interesting and useful results for a substantial majority of the test set apps. We choose to explain the results of two popular malware families, $A D R D$ and Geinimi. $A D R D$ is a family of malware that was wide-spread during the earlier versions of Android, had simple attack vectors to perform privacy leaks and

${ }^{13}$ Though Adagio, in principle could identify malice methods from CGs, the implementation provided at [19] does not include this. 


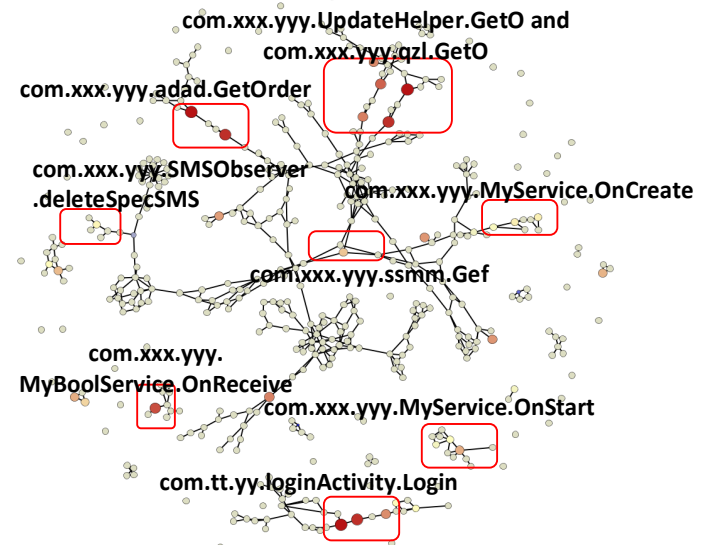

(a) API view

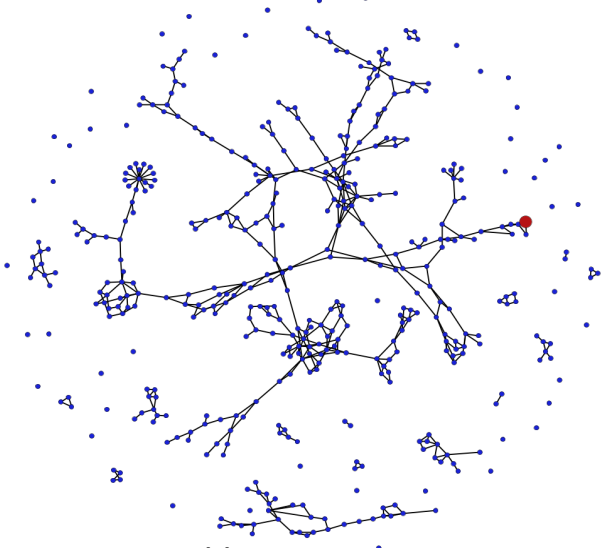

(c) Src-sink view

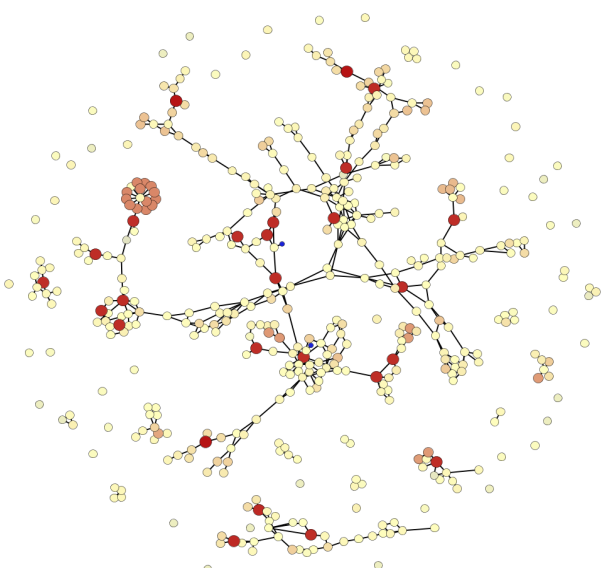

(e) Signature view

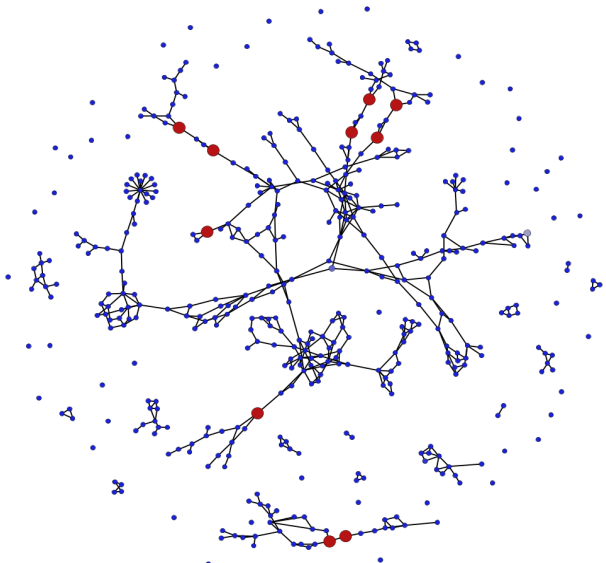

(b) Permission view

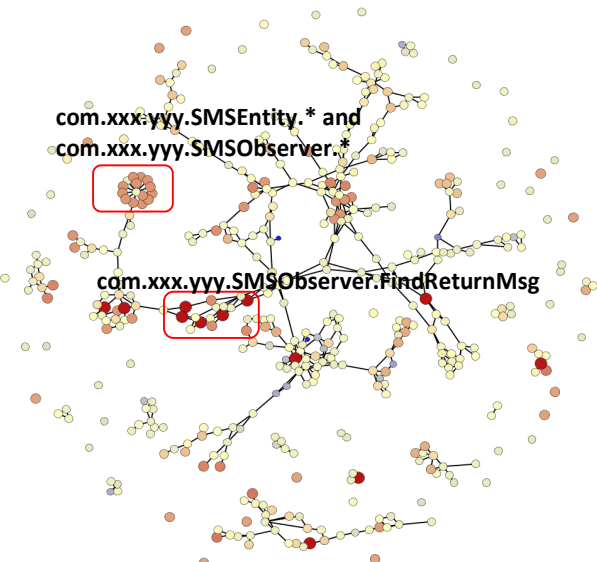

(d) Instruction view

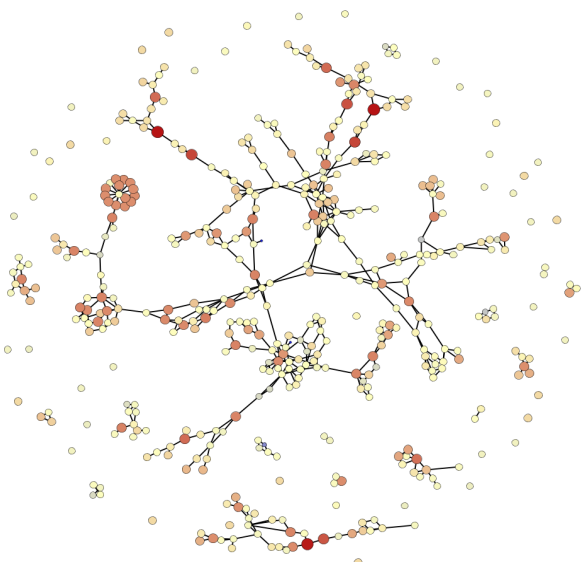

(f) MKLDroid - combined view

Figure 5.4: Depictions of CICFG of the ADRD sample from five different perspectives. (a) API view: the CICFG nodes are assigned $\mathrm{m}$-scores according to their CADG features i.e., API m-score (b) Permission view (c) Src-sink view (d) Instruction view (e) Signature view (f) MKL view: for every CICFG node, the $\mathrm{m}$-scores assigned are MKL based linear combinations of their individual views' m-scores. 
were not repackaged malware. Meaning, almost all the code in the $A D R D$ samples are mal-intended. On the other hand, Genimi performs more sensitive privacy leak attacks with more sophistication. Also, Geinimi samples in the DR dataset are repackaged versions of popular benign apps. Meaning, only a minority of the code in the Geinimi samples are mal-intended.

Case Study 1: ADRD. The ADRD sample ${ }^{14}$ used in this study attempts to perform the following malice actions in the background after the phone is booted: accessing users' personal sensitive information (e.g., IMEI, IMSI and network information) and sending them to remote servers, sending and deleting SMS messages, downloading unsolicited apps, and issuing HTTP search requests.

The five different views derived from the app's CICFG are presented in figures 5.4 (a) to (e). For the API kernel's view (in Fig. 5.4 (a)), the CICFG nodes are awarded m-scores based only the CADG contextual subgraph features that emerge from them. Similar mscore assignments are done to CICFG nodes in the remaining views in sub-figures (b) to (e). Finally, MKL based m-scores (which is a weighted sum of individual views' m-scores) are assigned to the CICFG nodes in Fig. 5.4 (f). The nodes are scaled in size and colored according to their m-score. Larger/warmer (i.e., reddish) nodes denote nodes with high mscores which are potentially malicious and smaller/cooler (i.e., blueish) denote nodes that supposedly do not involve in attack related activities.

The following observations are made from Fig. 5.4 (a):

- From API view, we could see that several basic blocks from classes such as com.xxx.yyy.MyService, com.xxx.yyy.MyBoolService and com.xxx.yyy.adad.GetOrder get high API m-scores. We inspected each of these methods manually and realized that these methods indeed perform malice activities. The precise dissection is presented below:

${ }^{14}$ MD5: 1944d8ee5bdda3a1bd06555fdb10d3267ab0cc4511d1e40611baf3ce1b81e5e8 
- Trigger. Similar to many popular malware families this ADRD sample uses broadcast notifications and alarm manager APIs to trigger its malicious operations. More specifically, method com.xxx.yyy.MyBoolService.OnReceive uses Intent.getBroadcast API to listen to a specific broadcast message notifying the BOOT_COMPLETED event. Once it receives the message, it sets an alarm that is fired periodically using the AlarmManager set API. These alarms would start a background service name MyService.

- Reading private data. MyService begins its lifecycle execution through invoking the com.xxx.yyy.MyService.OnCreate and com.xxx.yyy.MyService.OnStart methods. In the OnCreate method, the IMEI and IMSI numbers are collected by invoking getDeviceId and getSubsriberId APIs. Also, it registers an object handler to access the SMS database (content://sms/). The OnStart method collects some more private information such as network information (e.g., type of the network -'wifi' or 'UNIWAP') by invoking getActiveNetworkInfo and getTypeName APIs.

- Leaking private data. Once all this information is collected, the sample encrypts and leaks them over the internet. This is done in methods com.xxx.yyy.adad.GetOrder, com.xxx.yyy.UpdateHelper.GetO and com.xxx.yyy.qzl.GetO. The methods invoke sensitive APIs such as DefaultHttpClient.init, java.io.FileOutputStream.write and DefaultHttpClient.execute. Alternatively, the same information is exfiltrated through SMS using the APIs SmsManager.getDefault and sendTextMessage in method com.xxx.yyy.ssmm.Gef.

- Reading and deleting SMS. Besides this, the service uses method xxx.yyy.SMSObserver.deleteSpecSMS to monitor changes to the SMS database by calling ContentObserver.onChange API and deleting particular messages using ContentResolver. delete API.

- Downloading unsolicited apks. Moreover, the sample attempts to download a new 
unsolicited apk named 'myupdate.apk' and install the same on the device in method com.tt.yy.loginActivity.Login.

All these operations involved invoking sensitive APIs and hence are adequately captured in the API view. We now turn our attention to other remaining views. The following inferences from these perspectives in figures 5.4 (b) to (e):

- The Permission view (fig. 5.4 (b)) depicts the CICFG nodes scaled according to CPDG features based m-scores. As mentioned earlier, this ADRD sample's attack is simple and it turns out that only four permissions are required to carry out this, namely, READ_PHONE_STATE (for reading IMEI, IMSI etc.), ACCESS_NETWORK_STATE (for reading the network type), INTERNET(for leaking information through the network and communicating to $\mathrm{C} \& \mathrm{C}$ server) and SEND_SMS (for leaking information through SMS). Evidently, much less number of nodes correspond to code that uses these permissions and only these nodes get significant Permission m-scores. This is why we could see a lot of cooler/smaller nodes in this perspective. Out of the permissions, INTERNET is leveraged by almost all the ADRD variants for leaking information and are more popular than leaks via SMS. Consequently, the CPDG features related to former permission get higher m-scores than the latter ones. The features related to other two permissions are assigned lesser m-scores as well. Interestingly, the APIs used to read and delete SMS are not permission protected and hence they are assigned insignificant $\mathrm{m}$-scores. Overall, in this perspective, we have only a few suspicious nodes and they are from the following methods: com.xxx.yyy.UpdateHelper.GetO, com.xxx.yyy.adad.GetOrder, com.xxx.yyy.qzl.GetO and com.tt.yy.loginActivity.Login.

- The Src-sink view (fig. 5.4 (c)) depicts the CICFG nodes scaled according to Src-sink m-scores. There exists only one sensitive control flow path in this CICFG and it originates from com.xxx.yyy.MyService.OnCreate method. This path connects the source 
UNIQUE_IDENTIFIER to sinks FILE ${ }^{15}$ and SMS. We note that this sample leverages ICCs heavily. As our method does not capture ICCs and some paths that exist between other sources such as content resolver and FILE/SMS might be missed by our approach. Overall, in this perspective, we have only one suspicious node.

- Evidently, the Permission and Src-sink views over-abstract the semantics of the samples compared to the API view. Moreover, in the case of this sample, the information captured by the two former views is a supplementary to that captured in the API view. In other words, this example clearly reinforces the finding from RQ1 that the level of abstraction attained in the Permission and Src-sink views to too coarse-grained to be effective.

- As in figures 5.4 (d) and (e), the CICFGs scaled according to the Instruction and Signature m-scores and look very much different from the three aforementioned views. This is expected as those three views are closely related and capture similar semantics of the app. However, Instruction and Signature views, as discussed before, footprint the apps more like syntax-based detectors rather than semantics-based ones. For instance, the methods considered as significant in these two views are ones that belong to classes com.xxx.yyy.SMSEntity, com.xxx.yyy.SMSObserver. These two classes are present in the same composition in more than $27 \%$ of the ADRD samples in the DR dataset. These methods predominantly contain utilities code that is common across multiple variants of the ADRD family. Meaning, their CFG signatures and instruction sequences remain same across multiple samples and act as good features to characterize code that is unique in malware samples, but not necessarily malice. These two views precisely exploit these features to detect ADRD footprints. Figures 5.4 (d) and (e) visually illustrate that these two views capture information that is complementary to the three other views.

- Finally, we scale the nodes according to MKLDRoID's m-scores, which is nothing but

${ }^{15}$ In this context, the leaks through internet is considered akin to writing into a file and hence we see a FILE sink instead of a NETWORK sink. 
the linear combinations of their m-scores in each of the five views. Evidently, this multiview representation retains useful information from all the views. For instance, this view considers both com.xxx.yyy.UpdateHelper.GetO which is significant due to its semantic functionalities and com.xxx.yyy.SMSObserver.FindReturnMsg which is significant due to its popularity across multiple ADRD variants as more or less equally malicious.

- Finally, we note that this sample is not piggybacked. Meaning, much of the code (i.e., CICFG nodes) take part in the attack performing potentially harmful operations. This is indeed reflected well when we visualize MKLDRoID's results as most nodes are larger and warmer looking (i.e., assigned high m-scores).

Case Study 2: Ginmaster. The Geinimi sample ${ }^{16}$ used in this case study is the realworld version of the working example presented in §5.2. Unlike the ADRD sample, this is a repackaged malware. This sample's malice code is piggybacked on a popular benign game app. Also, the malicious functionalities in this sample are more sophisticated than ADRD. Geinimi's malice code is triggered through a system-generated broadcast message. On receiving it, Geinimi starts a service in the background. This service read volumes of personal data such as the users' location, contacts, emails and leaks them through internet and SMS messages.

The five different views derived from the app's CICFG are presented in figures 5.4 (a) to (e). At the outset, it is evident that unlike ADRD, across all the views, only a few nodes actually potentially malicious and have received high $\mathrm{m}$-scores. This is because in the case of this piggybacked Genimi app, only a few nodes involve in mal-intended operations (i.e., rider code) and the majority of the code is benign (i.e., host app's code).

The following observations are made from Fig. 5.5 (a):

- Trigger. On receiving the BOOT_COMPLETED event notification, a service named com.geinimi.AdService is started. This service continues to collect a variety of private ${ }^{16}$ MD5: 7bbd566f2f3abb78b3ffcc23ba4ad84e06a00f758d245c660c61b21814a850a5 


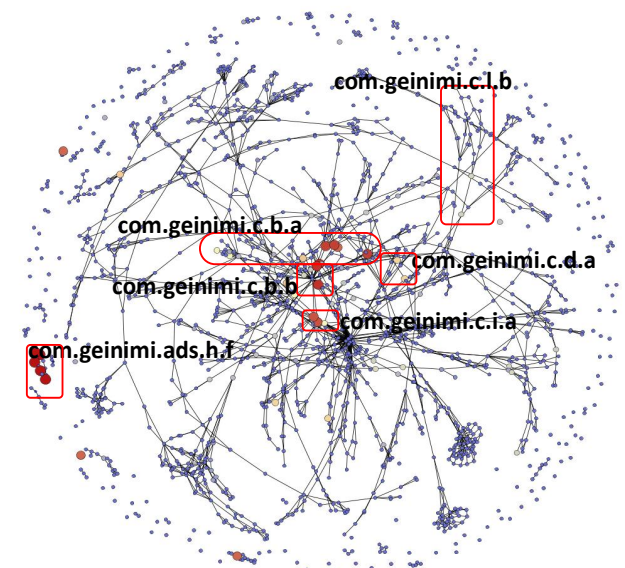

(a) ÁPI view

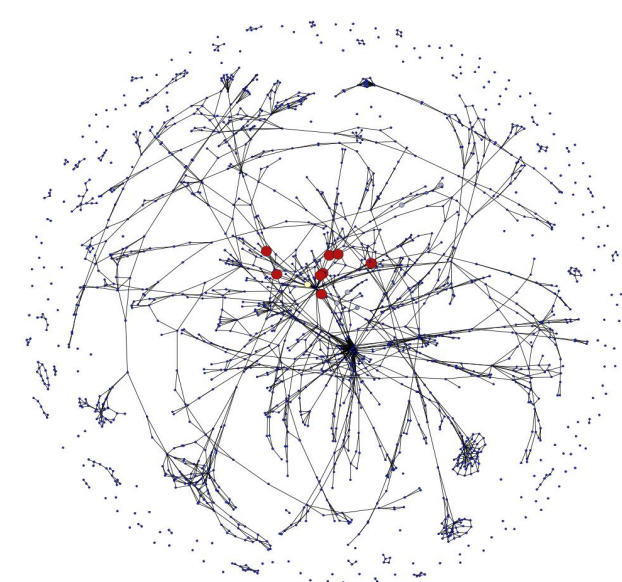

(c) Src-sink view

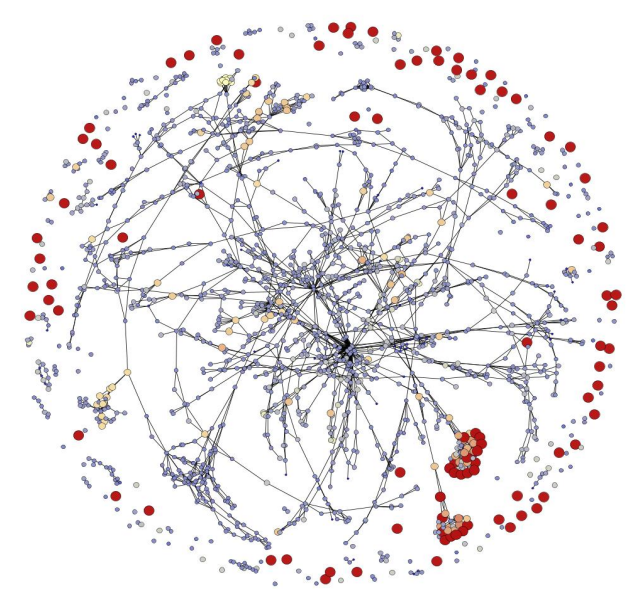

(e) Signature view

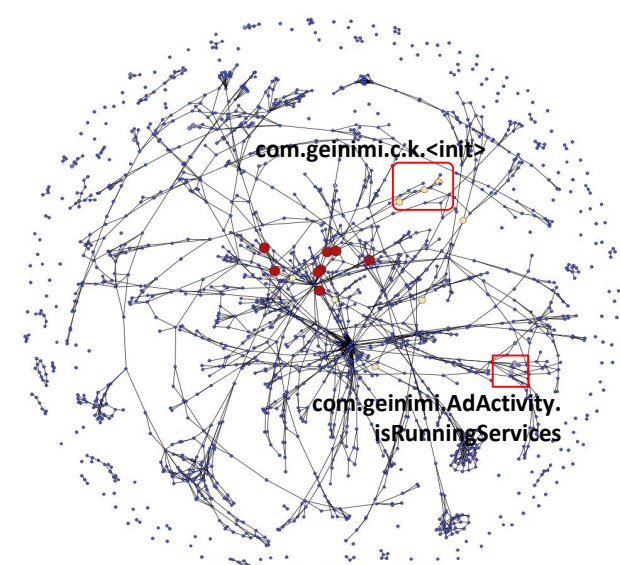

(b) Permission view

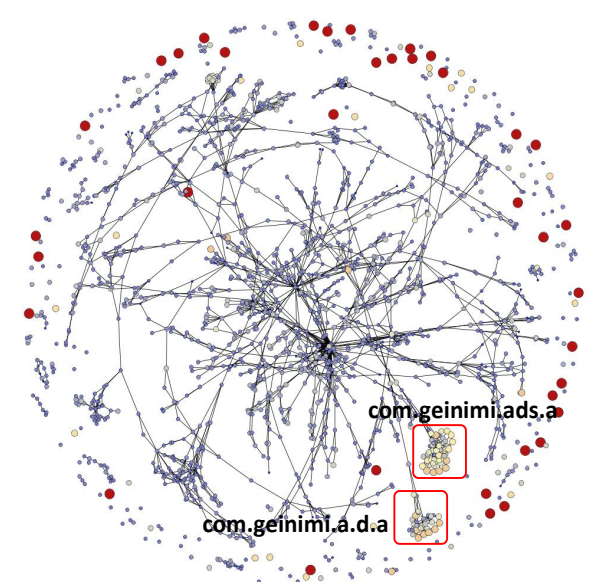

(d) Instruction view

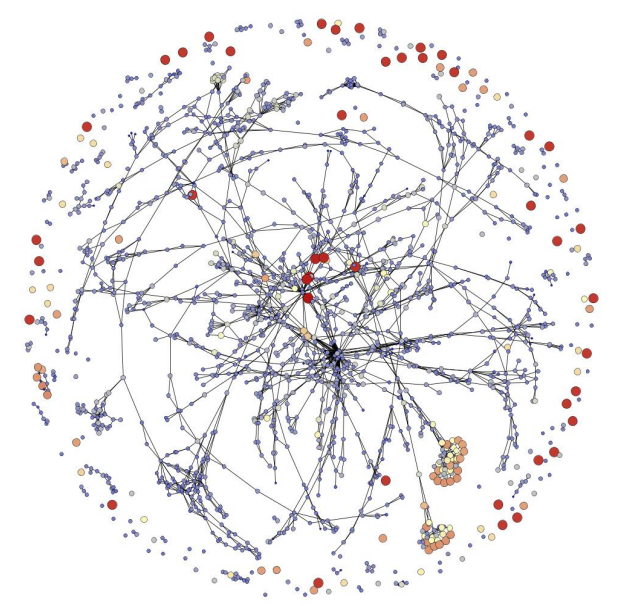

(f) MKLDroid - combined view

Figure 5.5: Depictions of CICFG of the Geinimi sample from five different perspectives. (a) API view: the ICFG nodes are assigned $\mathrm{m}$-scores according to their CADG features i.e., API m-score (b) Permission view (c) Src-sink view (d) Instruction view (e) Signature view (f) MKL view:for every CICFG node, the m-scores assigned are MKL based linear combinations of their individual views' m-scores. 
information and leaks the same as described below.

- Collecting location information. Geinimi collects user's geographic location in method geinimi.c.d.a using APIs getLastKnownLocation, getLati tude and getLongi tude.

- Collecting device identifiers. The method geinimi.c.k.init collects 13 different types of private information including phone number, IMEI, IMSI, mobile service operator's name, etc. using several APIs such as getLine1Number, getSimSerialNumber, getSimOperatorName, etc.

- Collecting contacts. This sample attempts to read users' contacts stored in the content providers through invoking getContentResolver and ContentResolver.query APIs. These operation are performed in methods geinimi.c.b.a and geinimi.c.b.b. The former method just reads the contact's display name, last contacted time and phone number. The latter method collects email addresses along with the above-mentioned information and bundles the same with the device's unique identifier making them ready to be leaked.

- Collecting emails related information. The emails stored in the content resolver are read using several methods in the class geinimi.ads.h and method $f$ in this class bundles the emails' to address, $c c / b c c$ address list, subject and content into an intent message (using ICC related APIs such as Intent.putExtra) and send them across to other methods for leaking them over internet.

- Leaking over internet and SMS. Finally, this sample leaks the collected private information over the internet in method geinimi.c.l.b using APIs such as java.net.HttpURLConnection.init, java.net.URL.init, URL.openconnection, java.io.DataOutputStream.write, flush, close and HttpURLConnection. disconnect. Alternatively, the same private contents are leaked through SMS in method geinimi.c.i.a. This method uses APIs SMSManager.getDefault and sendTextMessage. 
Similar to the case of $A D R D$ 's API view, all these operations in Geinimi involved invoking sensitive APIs and hence are adequately captured in this view. We now make the following observations from figures 5.5 (b) to (f):

- Similar to the Permission view in the previous case study, only a minority of Geinimi's APIs/URIs are permission protected and consequently, receive high Permission mscores. Predominantly, the following permissions are used by this sample to carry out the privacy leaks: ACCESS_COARSE_LOCATION, ACCESS_FINE_LOCATION, READ_CONTACTS, READ_PHONE_STATE, INTERNET and SEND_SMS. All these permissions are leveraged by the methods discussed above. Interestingly, since the method geinimi.c.k.init involves accessing a large number of device identifier related APIs across its different basic blocks, it happens to use READ_PHONE_STATE permission repeatedly. This behavior has rewarded more significant weights to these basic blocks in the Permission perspective than the CADG one. Also, another method, com.geinimi.AdActivity.isRunningServices, that helps to obfuscate Geinimi's attacks through using APIs such as Class. forName and getClassName. These are not permission protected APIs, however, we mapped them to special permissions (see §5.3.2) and this has yielded the corresponding nodes, more significant $\mathrm{m}$-scores in the Permission view compared to other views.

- Since Geinimi's privacy leaks involve more variety of information sources being read and leaked than ADRD, we could see several nodes with large m-scores in the Src-sink view in Fig. 5.5 (c). Typically, these control flow paths originate from the following methods: com.geinimi.c.b.a, com.geinimi.c.b.b and com.geinimi.c.d.a.

- Similar to the observations made in ADRD, both the Permission and Src-sink views over-abstract Geinimi's attacks. For instance, the sensitive operations in methods com.geinimi.ads.h.f and com.geinimi.c.l.b are not adequately captured in both these views. However, these two views could capture some critical information which is not reflected in API views. For instance, they exclusively reveal the sensitive operations in 
Table 5.9: MCLEx: Quantitative Evaluation of malicious code localization on the MYST dataset

\begin{tabular}{|l|c|c|c|c|}
\hline $\begin{array}{l}\text { Avg. \# of } \\
\text { malice classes }\end{array}$ & $\begin{array}{l}\text { FPR @ 10 } \\
\text { (avg. } \pm \text { std. })\end{array}$ & $\begin{array}{l}\text { FNR @ 10 } \\
\text { (avg. } \pm \text { std.) }\end{array}$ & $\begin{array}{l}\text { P @ 10 } \\
\text { (avg. } \pm \text { std.) }\end{array}$ & $\begin{array}{l}\text { R @ 10 } \\
\text { (avg. } \pm \text { std. })\end{array}$ \\
\hline 2.48 & $17.00( \pm 1.60)$ & $5.67( \pm 20.16)$ & $14.34( \pm 7.99)$ & $94.33( \pm 20.16)$ \\
\hline
\end{tabular}

methods such as com.geinimi.AdActivity.isRunningServices.

- Similar to the ADRD sample's case, the Instruction and Signature views footprint methods are statistically prominent across multiple variants of the Geinimi family. Specifcially, two methods com.geinimi.ads.a and com.geinimi.ads.b which figure in more than $15 \%$ of the Geinimi samples in the same composition are leveraged to footprint them in both these views.

- Finally, all the aforementioned views are integrated using MKL in Fig. 5.5 (f). Evidently, semantically significant nodes from methods such as com.geinimi.c.b.a and utility code related nodes from methods such as com.geinimi.ads.a receive high m-scores thus capturing the best of both worlds, helping effective detection. Overall, since this app is piggybacked with a large amount of benign code, one could a large number of cooler/smaller nodes in MKLDRoID's multi-view CICFG. This is in sharp contrast from ADRD's multi-view CICFG in Fig. 5.4 (f).

\section{Quantitative Evaluation}

We now present the results of quantitative evaluation of our experiments to locate malice code from MYST dataset. As mentioned earlier, in this experiment, the model is trained using 2,000 MYST malware and 2,000 benign apps. This model is then allowed to locate malice classes in each of the remaining 1,000 MYST apps. We consider classes with highest MKL based m-scores as malicious and compare them against the ground-truth on names of malice classes to compute the correctness and completeness of detection.

On average, we have 70.38 classes in MYST apps and according to ground truth, 2.48 
of them are malicious. Given this statistics, we consider that malice code is indeed present in top 10 classes with highest m-scores awarded by MKLDroID. Meaning for an analyst working on this dataset, MKLDroID would reduce the search space for seeing malice code by $1 / 7$ th of the total code, on average. FPR, FNR, precision and recall values averaged across all the 1,000 appss are presented in Table 5.9. The following inferences are drawn from the table:

- The average FNR is just over 5\% and the recall values is more than 94\%. Meaning, while inspecting only $1 / 7$ th of the code, MKLDroID facilitates the analysts to detect and inspect more than $94 \%$ of all the classes that are involved in malice operations. This is immensely helpful for analysts as it narrows down their search to potentially malice code locations.

- The average FPR and precision value is seemingly poor i.e., $17 \%$ and $14.34 \%$, respectively. The major reason for high FPR and low precision lies in the ratio between the number of actual and predicted malice classes. The average number of malice classes in the MYST dataset is as low as 2.48. However, remember, the malice code in MYST dataset is automatically generated and not very much close to the real-world scenario, where we would have a larger number of malice classes on average. For instance, even in the primitive $A D R D$ and Geinimi samples that were used in our case studies contained 32 and 82 malice classes, respectively. Given this statistics, in order to contain FNs, we have considered 10 classes with highest m-scores to be malice indeed. Since, the ratio of ground truth malice classes and predicted malice classes is very much skewed (i.e., 2.48/10), we obtain low average FPR and precision values. Furthermore, unlike automated detection, from an analysis view-point, 17\% of FPs is permissible. Meaning, while inspecting roughly $1 / 7$ th of the whole code, only $17 \%$ of the times MKLDroID would present a false positive class for review to the analyst. Just by reviewing the preliminary details such as name of the class (i.e., whether it is from libraries, or from host 
app and not the rider code), the analyst could quickly spot these FPs. Hence, given the nature of our application, low average FPR and precision could be justified.

Summarizing RQ3 inferences, it is clear that MKLDROID's individual views, owing to their inherent limitations, could only reveal a minority of malicious code portions. MKLDRoID achieves more comprehensive and precise malicious code localization results by appropriately combining the m-scores from individual views.

\subsection{Related Work}

Many well-known malware detection techniques have been reviewed in previous sections. We throw light on remaining works and contrast them from MKLDROID in this section under three categories: (1) PRG based approaches that use only one set of features, (2) approaches that use multiple feature-sets and (3) approaches that attempted coarse-grained malicious code localization.

\subsubsection{PRG based Android Malware Detection}

Structural features from PRGs (subgraphs, walks, etc.) have been used by a family of approaches reviewed below.

Adagio's structural detection and comparison has been already discussed in detail in $\S 3.5 .2$ and $\$ 5.5$. DroidMiner [31] proposes a two-tiered behavior graph to model malicious program logic into a vector of threat modalities, and then applies classification according to these modalities. DroidSIFT [29] models API-relevant behaviors into weighted CADGs and classifies malware based on a vocabulary of known malicious CADG subgraphs. However, unlike MKLDroid, DroidSIFT uses a fixed vocabulary of hand-picked subgraphs to construct feature vector representations. This limits the recall of the model when used over a longer period of time. Recently, AppContext [28] proposes differentiating malicious and 
benign behaviors based on the contexts similar to ours. However, it ends up capturing contextual features from individual nodes without their topological structural information. MassVet [34] statically analyzes apps' UI code to extract a graph that expresses UI states and transitions. Subsequently, it uses a DiffCom analysis to detect repackaged malware. MaMaDroid [33] constructs CGs, models API call sequences as Morkov Chain features and uses Instance-based classifiers (RFs and kNNs) for detection. Both MassVet and MaMaDroid do not capture contextual information.

On the other hand, a prominent set of works which leverage on PRGs for informationflow analysis include FlowDroid [53], IccTA [54], Mudflow [58] and DroidSafe [55]. These works predominantly use only the data-flow view and target detecting privacy leak attacks and related malware.

In sum, all the above-mentioned approaches capture PRGs' structural information from only one perspective, unlike MKLDROID.

\subsubsection{Multi-perspective Approaches}

Approaches leveraging on multiple features sets with different modalities have not been meagre, either. However, MKLDRoID differs from them in two ways: (i) none of them use context-aware or structural features which are very effective and (ii) the way it appropriately and systematically integrates multiple views.

Prominent works that employ multiple static and dynamic analysis-based feature-sets are discussed below. Drebin's methodology which uses as many as 8 feature-sets has been explained and compared in $§ 3.5 .2$ and $§ 5.5$. Sahs and Khan [38] extract a variety of features including tokens from user-defined permissions, standard permissions and CFG signatures and subsequently takes an anomaly detection approach using a One-Class SVM to detect malware. Peiravian et al. [42] take a simpler approach by considering permissions and API calls as features. MAST [39] uses selected permissions, Intent filters, the existence of na- 
tive code and zip files, then applies Multiple Correspondence Analysis to perform malware detection. MADAM [40] uses five feature-sets that includes system calls, critical APIs, user-interaction based features and metadata related features (e.g., rating, number of downloads, etc.). RevealDroid [59] uses four feature-sets namely, sensitive APIs, information flows, Intent actions and package-level API informations with Decision Tree classifier to perform detection. Recently, several techniques such as MARVIN [44], StatDynA [45] and StormDroid [47] adopt a hybrid approach by combining both static and dynamic analysis features and demonstrated achieving better accuracies than solutions leveraging on one of the analysis paradigms.

However, all the above-mentioned approaches just perform an early fusion of their multi-modal features i.e., just concatenate the feature vectors from individual perspectives. This results in obtaining a performance on par with uniform kernel of these features. As demonstrated in $§ 5.5 .1$, this results in sub-optimal accuracies. Unlike these approaches, thanks to its MKL phase, MKLDroID arrives at the most appropriate combination of its perspectives that offers best accuracy and explainability.

To the best of our knowledge, the only other work that uses MKL for multi-view Android malware detection is HADM [46]. MKLDRoID differs from this work in the following aspects: (i) HADM uses primitive features such as frequencies of APIs, advertisement network names etc. along with similar structural features. In other words, HADM does not use robust context-aware features like our approach, (ii) HADM uses a non-linear combination of base-kernels and hence its predictions are inexplainable, rendering it incapable of performing malicious code localization.

\subsubsection{Malicious Code Localization}

As mentioned earlier, multi-perspective malicious code localization is a unique feature of MKLDroID. However, we note that recently, two approaches have attempted to locate 
malice portion of PRGs. They are reviewed and contrasted below.

DrDroid [72]. This approach is specifically designed to detect repackaged malware and locate the injected malice code. DrDroid achieves this by splitting an app's CG into multiple regions called as DRegions and predicts whether each of them is malicious or benign. However, on many occasions, this method finds only one DRegion in apps, thus labeling all the code in an app as either malicious or benign. In fact, out 5,600 apps in DREBIN [25] dataset, this approach marked the entire code as malicious in 3,757 apps. Also, this approach could not rank portions of apps such as methods or classes based on their severity or degree of maliciousness. Meaning, all the code portions in the malice DRegion are considered equally malice.

HookRanker [71]. This approach is designed to identify piggybacked packages that are potentially malice from repackaged version of benign apps. HookRanker makes a strong assumption that malice code in repackaged malware will be contained in separate packages. While the validity of this assumption is debatable, this approach is incapable of identifying finer malice portions like methods and classes. As pointed out in [34] and [24], in many cases only certain classes and methods of the injected code are malice. In fact, HookRanker states "we consider all the injected code as malicious, even if the actual malicious payload is only some part of the added code". HookRanker is also incapable of discriminating code portions based on their degree of maliciousness.

Furthermore, in-principle, both these approaches are cannot detect malware that are not piggybacked.

In sum, none of the above-mentioned techniques exhibit all the three qualities that MKLDRoID possesses: context-aware, multi-view malware detection and malicious code localization. 


\subsection{Limitations}

Lack of data-flow and dynamic analyses. Our evaluations in RQ1 (§5.5.1) demonstrated MKLDroID's efficacy in detecting recent and complex malware in the wild. However, MKLDRoID, cannot generally detect all sorts of malicious behaviors, as it builds on concepts of static analysis and lacks dynamic inspection. Besides, information leak attacks identified by our approach are prone to false positive, as it takes into account only controlflow features and lacks data-flow analysis. As a natural extension of MKLDRoID, we intend to integrate these two perspectives in our future work. The reason behind not including them in the current work, is their poor scalability. As discussed in $[23,25,28,31,58]$ performing precise data-flow and dynamic analysis to extract features is computationally heavy.

In the current work, we mitigate the absence dynamic analysis features by maneuvering our multi-view analysis as follows: Permission view extracts API calls related to obfuscation, reflection, and loading of code, such as reflect.Constructor and DexClassLoader. loadClass and considers their invocations as use of special permissions. These CPDG features enable us to at least spot the execution of hidden code-even if we cannot further analyze it. In combinations with features from other views, MKLDRoID is still able to identify malicious behaviors despite the use of some obfuscation techniques.

Population drift. Recently, malware population drift (i.e., drift in malicious characteristics induced by malware evolution over time) has been studied closely by the research community and considered as serious and legitimate threat to practicality of malware detection techniques $[50,113]$. These works suggest using incremental ML techniques (e.g., online and active learning) to handle this drift, automatically. As noted through our evaluations in RQ 1.4 (\$5.5.1) MKLDRoID, being a batch-learning based framework, falls short of handling this drift and its recall keeps reducing over time. We intend to address this using 
Online MKL [93] approaches in the future.

Adversarial attacks. Another limitation which follows from the use of ML is the possibility of attacks by adversaries such as poisoning (see $[66,90])$. While common obfuscation strategies, such as identifier renaming and code reordering do not affect MKLDROID, adversaries may succeed in reducing its accuracy by incorporating benign contextual subgraph features or fake invariants into malicious apps. Even though such adversarial attacks against ML based detectors cannot be ruled out in general, meticulous sanitization of training data (see [66]) can limit their impact.

\subsection{Conclusions}

In this chapter, we propose MKLDRoID, a framework that performs context-aware, multiview malware detection and malicious code localization. In its pipeline of processes, firstly, MKLDRoID deploys a graph kernel that is capable of capturing both contextual and structural information from PRGs to build explicit feature vectors representing five different semantic views of apps. Subsequently, it combines these views in a systematic and scalable manner using MKL and performs detection. Finally, as its unique feature, after detection, MKLDroID uses a novel kernel methods based approach to award m-scores to every PRG node which quantifies the degree of malice activities it performs. This helps to precisely locate malice code portions (i.e., malice classes/methods) in PRGs. Through our large-scale experiments on both benchmark and wild dataset apps, we demonstrate that MKLDRoID, significantly outperforms state-of-the-art techniques in terms of accuracy (i.e., by more than $11 \%$ F-measure on real-world experimental settings), while maintaining high efficiency. Also, in malicious code localization experiments, it identifies all the malice classes in apps with $94 \%$ average recall.

Release of results. In order to provide scope for persuasive research on malicious code localization, we release the result of qualitative evaluation of all the apps in DREBIN [25] 
dataset at https://sites.google.com/site/mkldroid. This dataset contains the ICFGs of all the apps. Each node is assigned an m-score quantifying its degree of maliciousness. Additionally, for every node, sensitive APIs accessed, permissions, sources, sinks used, instruction sequences and CFG signatures are also provided as node attributes. This helps to gain better insights on code portions, similar to the ones presented in our case studies (see 5.5.3). ICFGs (with aforementioned node attributes) of 1000 MYST apps used in our quantitative evaluation in $\S 5.5 .3$ are also released. 


\section{Chapter 6}

\section{SubGRAPH2vec: Learning Distributed}

\section{Representations of Rooted Sub-graphs}

\section{from Large Graphs}

In this chapter, SUBGRAPH2VEC, an unsupervised representation learning technique to learn embeddings of subgraphs is presented. The contents of this chapter are presented in a KDD 2016 workshop paper titled "SuBgRAPH2vec: Learning Distributed Representations of Rooted Sub-graphs from Large Graphs". Also, an extension of this work titled "Asum: A Deep Learning based Framework for Malware Attack Summarization and Familial Analysis" is under preparation.

\subsection{Introduction}

As it could be seen from previous chapters, our kernel, CWLK is not a general purpose graph kernel. It is specifically designed to cater an application, namely, malware detection. Meaning, it could not be used for measuring similarities between arbitrary graphs. This renders CWLK unusable to address the second application that we consider in this thesis, namely, clone detection. The following are the fundamental differences between the malware and clone detection tasks: (1) malware detection works by training a model to 
distinguish malice and benign code patterns, whereas clone detection just involves identifying similar code patterns, and (2) for malware detection, we need a collection of known benign and malware apps, whereas for clone detection, we just need a collection of unlabeled apps. In essence, clone detection could be considered as an unsupervised learning (clustering) task, whereas malware detection is a supervised learning (classification) task. On the other hand, it could be seen that the primary similarity between these two tasks is, they both work by estimating the similarities between specific code patterns. Hence, one could use a general purpose graph kernel such as WLK which could help measure the code pattern similarities and perform both clone and malware detection leveraging on an appropriate learning paradigm. In fact, recently, Li et al. [122] demonstrated using WLK for clone detection and Shas et al. [38] demonstrated using the same for malware detection. To this end, in this chapter, we propose a general purpose graph kernel that could be used to measure similarities among PRG patterns and thereby could cater both clone and malware detection. Our goal in this chapter is to overcome some of the shortcomings in the existing kernels with the intention of improving their accuracies in both the above said tasks. However, it is noted that this kernel will not have a problem specific flavor, such as capturing reachability contexts from PRGs.

From here onwards, we proceed with proposing our general purpose kernel and demonstrate its efficacy in several graph classification and clustering task, across several domains.

\subsubsection{Graph Kernels}

In general, graphs offer a rich, generic and natural way for representing structured data. In domains such as computational biology, chemoinformatics, social network analysis and program analysis, we are often interested in computing similarities between graphs to cater domain-specific applications such as protein function prediction, drug toxicity prediction 


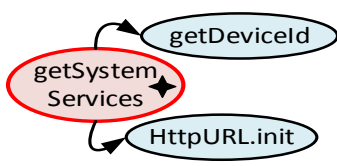

(a)

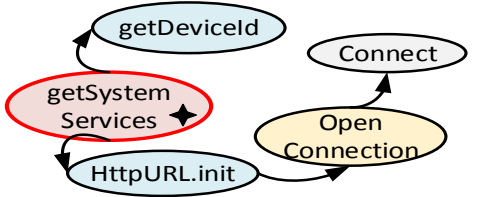

(c)

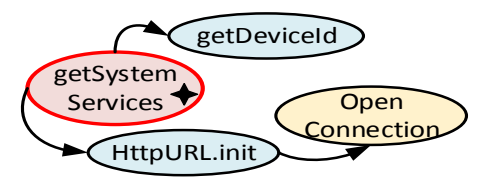

(b)

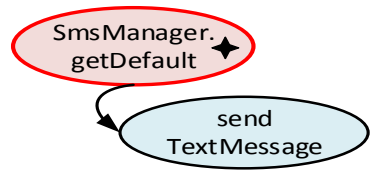

(d)

Figure 6.1: Dependency schema of a set of rooted subgraphs of degree 1 ((a), (d)), 2 ((b)) and 3 ((c)) in an Android malware's API dependency graph. The root nodes are marked with a star. Graph (b) can be derived from (a) by adding a node and an edge. Graph (c) can be derived from (b) in a similar fashion. Graph (d) is highly dissimilar from all the other graphs and is not readily derivable from any of them.

and malware detection.

Graph Kernels are one of the popular and widely adopted approaches to measure similarities among graphs $[76,77,85,99,106]$. A Graph kernel measures the similarity between a pair of graphs by recursively decomposing them into atomic substructures (e.g., walk [99], shortest paths [76], graphlets [85] etc.) and defining a similarity function over the substructures (e.g., number of common substructures across both graphs). This makes the kernel function correspond to an inner product over substructures in reproducing kernel Hilbert space (RKHS). Formally, for a given graph $G$, let $\Phi(G)$ denote a vector which contains counts of atomic substructures, and $\langle\cdot, \cdot\rangle_{H}$ denote a dot product in a RKHS $H$. Then, the kernel between two graphs $G$ and $G^{\prime}$ is given by

$$
k\left(G, G^{\prime}\right)=\left\langle\Phi(G), \Phi\left(G^{\prime}\right)\right\rangle_{H}
$$

From an application standpoint, the kernel matrix $\mathbf{k}$ that represents the pairwise similarity of graphs in the dataset (calculated using eq. (6.1)) could be used in conjunction with kernel classifiers (e.g., SVM) and relational data clustering algorithms (e.g., Affinity Propagation (AP) [108]) to perform graph classification and clustering tasks, respectively. 


\subsubsection{Limitations of Existing Graph Kernels}

However, as noted in $[85,106]$, existing kernels with the representation as in eq. (6.1) does not take two important observations into account.

- (L1) Substructure Similarity. Substructures that are used to compute the kernel matrix are not independent. To illustrate this, lets consider WLK [77] which decomposes graphs into rooted subgraphs ${ }^{1}$. These subgraphs encompass the neighbourhood of certain degree around the root node. Understandably, these subgraphs exhibit strong relationships among them. That is, a subgraph with second degree neighbours of the root node could be arrived at by adding a few nodes and edges to its first degree counterpart. We explain this with an example presented in Fig. 6.1. The figure illustrates API-dependency subgraphs from a well-known Android malware called DroidKungFu (DKF) [25]. These subgraph portions of DKF involves in leaking users' private information (i.e., IMEI number) over the internet and sending premium-rates SMS without her consent. Subfigures (a), (b) and (c) represent subgraphs of degree 1, 2 and 3 around the root node getSystemServices, respectively. Evidently, these subgraphs exhibit high similarity among one another. For instance, subgraph (c) could be derived from subgraph (b) by adding a node and an edge, which in turn could be derived from subgraph (a) in a similar fashion. However, the WL kernel, by design ignores these subgraph similarities and considers each of the subgraphs as individual features. Other kernels such as RW and SP kernels also make similar assumptions on their respective substructures' similarities.

- (L2) Diagonal Dominance. Since graph kernels regard these substructures as separate features, the dimensionality of the feature space often grows exponentially with the number of substructures. Consequently, only a few substructures will be common across graphs. This leads to diagonal dominance, that is, a given graph is similar to itself but

${ }^{1}$ The WL kernel models the subgraph around a root node as a tree (i.e., without cycles) and hence is referred as WL subtree kernel. However since the tree represents a rooted subgraph, we refer to the rooted subgraph as the substructure being modeled in WL kernel, in this work. 
not to any other graph in the dataset. This leads to poor generalization and thereby to poor accuracy.

\subsubsection{Existing Solution: Deep Graph Kernels}

To alleviate these problems Yanardag and Vishwanathan [85], recently proposed an alternative kernel formulation termed as Deep Graph Kernel (DGK). Unlike eq. (6.1), DGK captures the similarities among the substructures with the following formulation:

$$
\mathcal{K}\left(G, G^{\prime}\right)=\Phi(G)^{T} \mathcal{M} \Phi\left(G^{\prime}\right)
$$

where $\mathcal{M}$ represents a $|\mathcal{V}| \times|\mathcal{V}|$ positive semi-definite matrix that encodes the relationship between substructures and $\mathcal{V}$ represents the vocabulary of substructures obtained from the training data. Therefore, one can design a $\mathcal{M}$ matrix that respects the similarity of the substructure space.

Learning representation of substructures. In DGK [85], the authors used representation learning (deep learning) techniques inspired by the work of Mikolov et al. [107] to learn vector representations (i.e., embeddings) of substructures. Subsequently, these substructure embeddings were used to compute $\mathcal{M}$ and the same is used in eq (6.2) to arrive at the deep learning variants of several well-known kernels such as WL, graphlet and SP kernels.

Context. In order to facilitate unsupervised representation learning on graph substructures, the authors of [85] defined a notion of context among these substructures. Substructures that co-occur in the same context tend to have high similarity. For instance, in the case of rooted subgraphs, all the subgraphs that encompass same degree of neighbourhood around the root node are considered as co-occurring in the same context (e.g., all degree-1 subgraphs are considered to be in the same context). Subsequently, embedding learning 
task's objective is designed to make the embeddings of substructures that occur in the same context similar to one another. Thus defining the correct context is of paramount importance to build high quality embeddings.

Deep WL Kernel. Through their experiments the authors demonstrated that the deep learning variant of WL kernel constructed using the above-said procedure achieved stateof-the-art performances on several datasets. However, we observe that, in their approach to learn subgraph embeddings, the authors make three novice assumptions that lead to three critical problems:

- (A1) Only rooted subgraphs of same degree are considered as co-occurring in the same context. That is, if $D_{G}^{(d)}=\left\{\left\{s g_{1}^{(d)}, s g_{2}^{(d)}, \ldots\right\}\right\}$ is a multi-set of all degree $d$ subgraphs in graph G, [85] assumes that any two subgraphs $s g_{i}^{(d)}, s g_{j}^{(d)} \in D_{G}^{(d)}$ co-occur in the same context irrespective of the length (or number) of path(s) connecting them or whether they share the same nodes/edges. For instance, in the case of Android malware subgraphs in Fig. 6.1, [85] assumes that only subgraphs (a) and (d) are in the same context and are possibly similar as they both are degree-1 subgraphs. However in reality, they share no APIs in common and are highly dissimilar. This assumption makes subgraphs that do not co-occur in the same graph neighbourhood to be in the same context and thus similar (problem 1).

- (A2) Any two rooted subgraphs of different degrees never co-occur in the same context. That is, two subgraphs $s g_{i}^{(d)} \in D_{G}^{(d)}$ and $s g_{j}^{\left(d^{\prime}\right)} \in D_{G}^{\left(d^{\prime}\right)}$ (where $d \neq d^{\prime}$ ) never co-occur in the same context irrespective of the length (or number) of path(s) connecting them or whether they share the same nodes/edges. For instance, in Fig. 6.1, subgraphs (a), (b) and (c) are considered not co-occurring in the same context as they belong to different degree neighbourhood around the root node. Hence, [85] incorrectly biases them to be dissimilar. This assumption makes subgraphs that co-occur in the same neighbourhood not to be in the same context and thus dissimilar (problem 2). 
- (A3) Every subgraph $\left(s g_{r}^{(d)}\right)$ in any given graph has exact same number of subgraphs in its context, which is equal to the number of nodes in $G$. This assumption clearly violates the topological neighbourhood structure in graphs (problem 3).

Through our thorough analysis and experiments we observe that these assumptions led [85] to building relatively low quality subgraph embeddings. Consequently, this reduces the classification and clustering accuracies when [85]'s deep learning variant of WLK is deployed. This motivates us to address these limitations and build better subgraph embeddings, in order to achieve higher accuracy.

\subsubsection{Our Approach}

In order to learn accurate subgraph embeddings, we address each of the three problems introduced in the previous subsection. We make two main contributions through our SuBGRAPH2VEC framework to solve these problems:

- We extend the WL relabeling strategy [77] (used to relabel the nodes in a graph encompassing its breadth-first neighbourhood) to define a proper context for a given subgraph. For a given subgraph $s g_{r}^{(d)}$ in $G$ with root $r$, SUBGRAPH2VEC considers all the rooted subgraphs (up to a certain degree) of neighbours of $r$ as the context of $s g_{r}^{(d)}$. This solves problems 1 and 2 (mentioned in $§ 6.1 .3$ ).

- However this context formation procedure yields radial contexts of different sizes for different subgraphs. This renders the existing representation learning models such as the skipgram model [107] (which captures fixed-length linear contexts) unusable in a straight-forward manner to learn the representations of subgraphs using its context, thus formed. To address this we propose a modification to the skipgram model enabling it to capture varying length radial contexts. This solves problem 3.

Experiments. We determine SubGRAPH2vec's accuracy and efficiency in both super- 
vised and unsupervised learning tasks with several benchmark and large-scale real-world datasets. Also, we perform comparative analysis against several state-of-the-art graph kernels. Our experiments reveal that Subgraph2Vec achieves significant improvements in classification/clustering accuracy over existing kernels. Specifically, on two real-world program analysis tasks, namely, code clone and malware detection, SubGRAPH2vec outperforms state-of-the-art kernels by more than $17 \%$ and $4 \%$, respectively.

Contributions. We make the following contributions in this chapter:

- We propose Subgraph2vec, an unsupervised representation learning technique to learn latent representations of rooted subgraphs present in large graphs (§6.5).

- We develop a modified version of the skipgram language model [107] which is capable of modeling varying length radial contexts (rather than fixed-length linear contexts) around target subgraphs $(\S 6.5 .2)$.

- We discuss how Subgraph2vec's representation learning technique would help to build the deep learning variant of WL kernel (§6.5.3).

- Through our large-scale experiments on several benchmark and real-world datasets, we demonstrate that SuBgRAPH2vec could significantly outperform state-of-the-art graph kernels (incl. [85]) on graph classification and clustering tasks (§6.6).

\subsection{Related Work}

The closest work to ours is Deep Graph Kernels [85]. Since we have discussed it elaborately in $\$ 6.1$, we refrain from discussing it here. Recently, there has been significant interest from the research community on learning representations of nodes and other substructures from graphs. We list the prominent such works in Table 6.1 and show how our work compares to them in-principle. Deep Walk [101] and node2vec [103] intend to learn 
Table 6.1: Representation Learning from Graphs

\begin{tabular}{|c|c|c|c|l|}
\hline Solution & $\begin{array}{c}\text { Learning } \\
\text { Paradigm }\end{array}$ & $\begin{array}{c}\text { node } \\
\text { vector }\end{array}$ & $\begin{array}{c}\text { subgraph } \\
\text { vector }\end{array}$ & $\begin{array}{l}\text { Context used for } \\
\text { rep. learning }\end{array}$ \\
\hline Deep Walk [101] & Semi-sup & - & $\bigcirc$ & $\begin{array}{l}\text { Fixed-length } \\
\text { random walks }\end{array}$ \\
\hline node2vec [103] & Semi-sup & - & $\bigcirc$ & $\begin{array}{l}\text { Fixed-Length biased } \\
\text { random walks }\end{array}$ \\
\hline Patchy-san [102] & Sup & - & - & $\begin{array}{l}\text { Receptive field of sequence } \\
\text { of neighbours of nodes }\end{array}$ \\
\hline $\begin{array}{l}\text { Deep Graph [85] } \\
\text { Kernels }\end{array}$ & Unsup & - & $\wp$ & $\begin{array}{l}\text { Subgraphs occurring } \\
\text { at same degree }\end{array}$ \\
\hline SubGRAPH2vec & Unsup & - & $\bigcirc$ & $\begin{array}{l}\text { Subgraphs of different } \\
\text { degrees occurring in the } \\
\text { same local neighbourhoods }\end{array}$ \\
\hline
\end{tabular}

node embeddings by generating random walks in a single graph. Both these works rely on existence of node labels for at least a small portion of nodes and take a semi-supervised approach to learn node embeddings. Recently proposed Patchy-san [102] learns node and subgraph embeddings using a supervised CNN based approach. In contrast to these three works, SuBgRAPH2vec learns subgraph embeddings (which includes node embeddings) in an unsupervised manner.

In general, from a substructure analysis point of view, research on graph kernel could be grouped into three major categories: kernels for limited-size subgraphs [104], kernels based on subtree patterns [77] and kernels based on walks [99] and paths [76]. SUBGRAPH2vec is complementary to these existing graph kernels where the substructures exhibit reasonable similarities among them.

\subsection{Problem Statement}

We consider the problem of learning distributed representations of rooted subgraphs from a given set of graphs. More formally, let $G=(V, E, \lambda)$, represent a graph, where $V$ is a 
set of nodes and $E \subseteq(V \times V)$ be a set of edges. Graph $G$ is labeled ${ }^{2}$ if there exists a function $\lambda$ such that $\lambda: V \rightarrow \ell$, which assigns a unique label from alphabet $\ell$ to every node $v \in V$. Given $G=(V, E, \lambda)$ and $s g=\left(V_{s g}, E_{s g}, \lambda_{s g}\right), s g$ is a sub-graph of $G$ iff there exists an injective mapping $\mu: V_{s g} \rightarrow V$ such that $\left(v_{1}, v_{2}\right) \in E_{s g}$ iff $\left(\mu\left(v_{1}\right), \mu\left(v_{2}\right)\right) \in E$.

Given a set of graphs $\mathcal{G}=\left\{G_{1}, G_{2}, \ldots, G_{n}\right\}$ and a positive integer $D$, we intend to extract a vocabulary of all (rooted) subgraphs around every node in every graph $G_{i} \in \mathcal{G}$ encompassing neighbourhoods of degree $0 \leq d \leq D$, such that $S G_{\text {vocab }}=\left\{s g_{1}, s g_{2}, \ldots\right\}$. Subsequently, we intend to learn distributed representations with $\delta$ dimensions for every subgraph $\mathrm{sg}_{i} \in S G_{\text {vocab. }}$. The matrix of representations (embeddings) of all subgraphs is denoted as $\Phi \in \mathbb{R}^{\left|S G_{\text {vocab }}\right| \times \delta}$.

Once the subgraph embeddings are learnt, they could be used to cater applications such as graph classification, clustering, node classification, link prediction and community detection. They could be readily used with classifiers such as CNNs and Recurrent Neural Networks. Besides this, these embeddings could be used to make a graph kernel (as in eq. (6.2)) and subsequently used along with kernel classifiers such as SVMs and relational data clustering algorithms. These use cases are elaborated later in $§ 6.5 .4$ after introducing the representation learning methodology.

\subsection{Background: Language Models}

Our goal is to learn the distributed representations of subgraphs extending the recently proposed representation learning and language modeling techniques for multi-relational data. In this section, we review the related background in language modeling.

Traditional language models. Given a corpus, the traditional language models determine the likelihood of a sequence of words appearing in it. For instance, given a sequence of words $\left\{w_{1}, w_{2}, \ldots, w_{T}\right\}, \mathrm{n}$-gram language model targets to maximize the following proba${ }^{2}$ For graphs without node labels, we follow the procedure mentioned in [77] and label nodes with their degree. 
bility:

$$
\operatorname{Pr}\left(w_{t} \mid w_{1}, \ldots, w_{t-1}\right)
$$

Meaning, they estimate the likelihood of observing the target word $w_{t}$ given $n$ previous words $\left(w_{1}, \ldots, w_{t-1}\right)$ observed thus far.

Neural language models. The recently developed neural language models focus on learning distributed vector representation of words. These models improve traditional ngram models by using vector embeddings for words. Unlike n-gram models, neural language models exploit the of the notion of context where a context is defined as a fixed number of words surrounding the target word. To this end, the objective of these word embedding models is to maximize the following log-likelihood:

$$
\sum_{t=1}^{T} \log \operatorname{Pr}\left(w_{t} \mid w_{t-c}, \ldots, w_{t+c}\right)
$$

where $\left(w_{t} \mid w_{t-c}, \ldots, w_{t+c}\right)$ are the context of the target word $w_{t}$. Several methods are proposed to approximate eq. (6.4). Next, we discuss one such a method that we extend in our SUBGRAPH2vec framework, namely Skipgram models [107].

\subsubsection{Skip Gram}

The skipgram model maximizes co-occurrence probability among the words that appear within a given context window. Give a context window of size $c$ and the target word $w_{t}$, skipgram model attempts to predict the words that appear in the context of the target word, $\left(w_{t-c}, \ldots, w_{t-c}\right)$. More precisely, the objective of the skipgram model is to maximize the following loglikelihood,

$$
\sum_{t=1}^{T} \log \operatorname{Pr}\left(w_{t-c}, \ldots, w_{t+c} \mid w_{t}\right)
$$


where the probability $\operatorname{Pr}\left(w_{t-c}, \ldots, w_{t+c}\right)$ is computed as

$$
\Pi_{-c \leq j \leq c, j \neq 0} \operatorname{Pr}\left(w_{t+j} \mid w_{t}\right)
$$

Here, the contextual words and the current word are assumed to be independent. Furthermore, $\operatorname{Pr}\left(w_{t+j} \mid w_{t}\right)$ is defined as:

$$
\frac{\exp \left(\Phi_{w_{t}}^{T} \Phi_{w_{t+j}}^{\prime}\right)}{\sum_{w=1}^{\mathcal{V}} \exp \left(\Phi_{w_{t}}^{T} \Phi_{w}^{\prime}\right)}
$$

where $\Phi_{w}$ and $\Phi_{w}{ }^{\prime}$ are the input and output vectors of word $w$.

\subsubsection{Negative Sampling}

The posterior probability in eq. (6.6) could be learnt in several ways. For instance, a novice approach is to use a classifier like logistic regression. This is prohibitively expensive if the vocabulary of words is very large.

Negative sampling is an efficient algorithm that is used to alleviate this problem and train the skipgram model. Negative sampling selects the words that are not in the context at random instead of considering all words in the vocabulary. In other words, if a word $w$ appears in the context of another word $w^{\prime}$, then the vector embedding of $w$ is closer to that of $w^{\prime}$ compared to any other randomly chosen word from the vocabulary.

Once skipgram training converges, semantically similar words are mapped to closer positions in the embedding space revealing that the learned word embeddings preserve semantics. An important intuition we extend in Subgraph2vec is to view subgraphs in large graphs as words that are generated from a special language. In other words, different subgraphs compose graphs in a similar way that different words form sentences when used together. With this analogy, one can utilize word embedding models to learn dimensions of similarity between subgraphs. The main expectation here is that similar subgraphs will 
be close to each other in the embedding space.

\subsection{Method: Learning Sub-graph Representations}

In this section we discuss the main components of our SUBGRAPH2vEc algorithm (§6.5.2), how it enables making a deep learning variant of WL kernel (§6.5.3) and some of its usecases in detail ( $\$ 6.5 .4)$.

\subsubsection{Overview}

Similar to the language modeling convention, the only required input is a corpus and a vocabulary of subgraphs for SUBGRAPH2vec to learn representations. Given a dataset of graphs, SubGRAPH2VEc considers all the neighbourhoods of rooted subgraphs around every rooted subgraph (up to a certain degree) as its corpus, and set of all rooted subgraphs around every node in every graph as its vocabulary. Subsequently, following the language model training process with the subgraphs and their contexts, SubGraph2vec learns the intended subgraph embeddings.

\subsubsection{Algorithm: SubGraPH2veC}

The algorithm consists of two main components; first a procedure to generate rooted subgraphs around every node in a given graph and second the procedure to learn embeddings of those subgraphs.

As presented in Algorithm 5 we intend to learn $\delta$ dimensional embeddings of subgraphs (up to degree $D$ ) from all the graphs in dataset $\mathcal{G}$ in e epochs. We begin by building a vocabulary of all the subgraphs, $S G_{\text {vocab }}$ (line 2) (using Algorithm 6). Then the embeddings for all subgraphs in the vocabulary $(\Phi)$ is initialized randomly (line 3 ). Subsequently, we proceed with learning the embeddings in several epochs (lines 4 to 10) iterating over the 


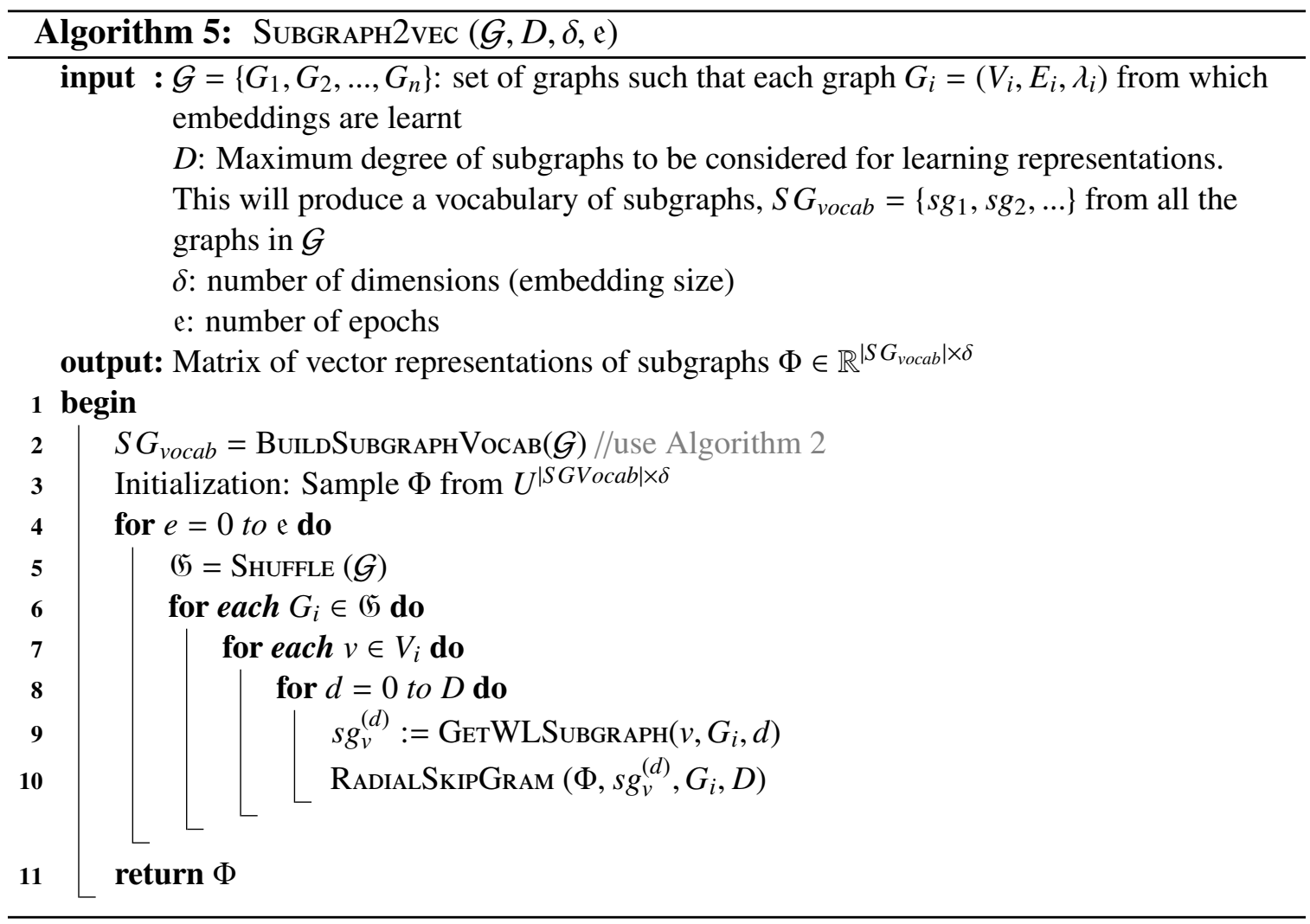

graphs in $\mathcal{G}$. These steps represent the core of our approach and are explained in detail in the two following subsections.

\section{Extracting Rooted Subgraphs}

To facilitate learning its embeddings, a rooted subgraph $s g_{v}^{(d)}$ around every node $v$ of graph $G_{i}$ is extracted (line 9). This is a fundamentally important task in our approach. To extract these subgraphs, we follow the well-known WL relabeling process [77] which lays the basis for the WL kernel and WL test of graph isomorphism [77, 85]. The subgraph extraction process is explained separately in Algorithm 6. The algorithm takes the root node $v$, graph $G$ from which the subgraph has to be extracted and degree of the intended subgraph $d$ as inputs and returns the intended subgraph $s g_{v}^{(d)}$. When $d=0$, no subgraph needs to be extracted and hence the label of node $v$ is returned (line 3 ). For cases where $d>0$, we get all the (breadth-first) neighbours of $v$ in $\mathcal{N}_{v}$ (line 5). Then for each neighbouring node, $v^{\prime}$, 

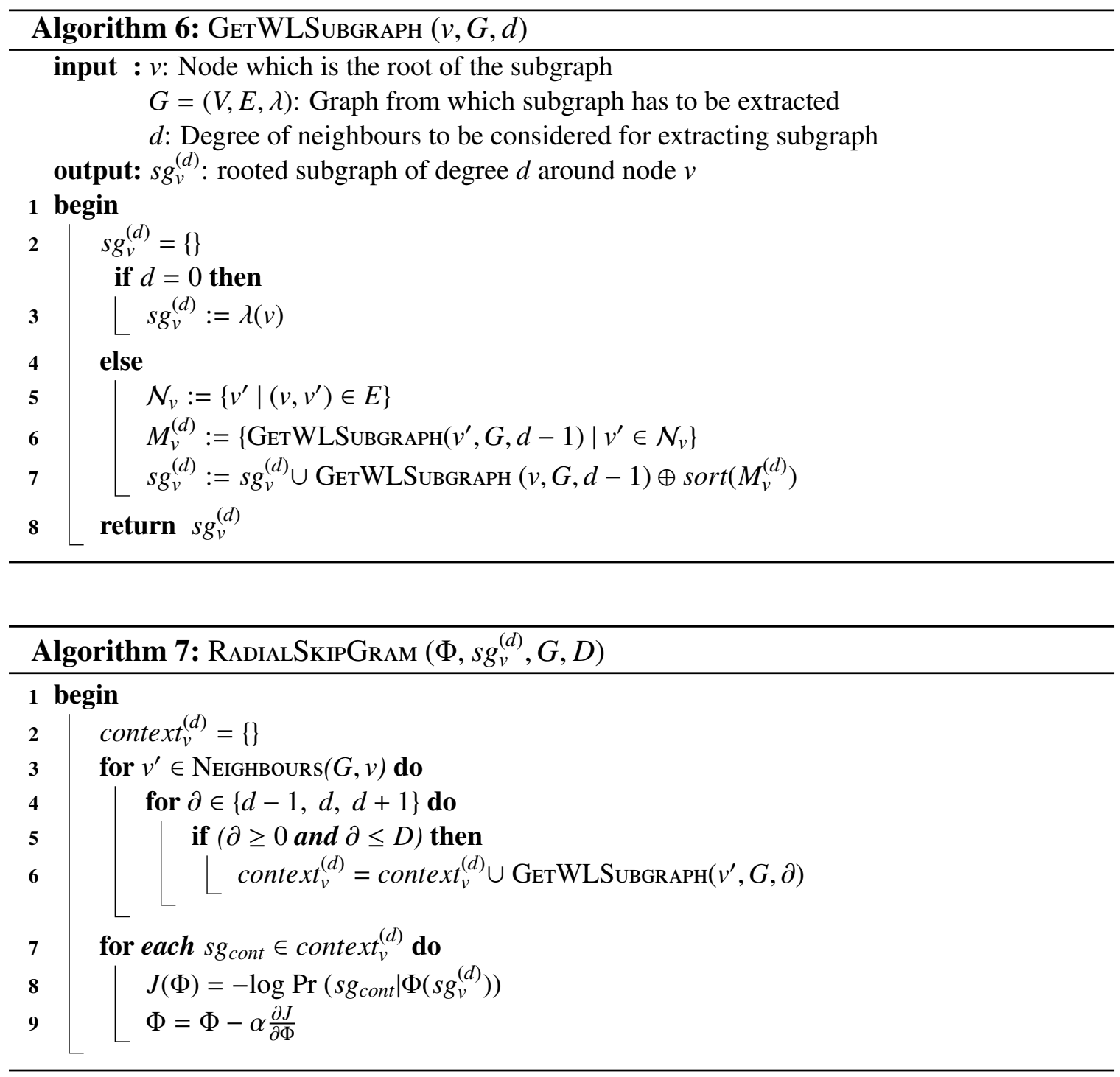

we get its degree $d-1$ subgraph and save the same in list $M_{v}^{(d)}$ (line 6). Finally, we get the degree $d-1$ subgraph around the root node $v$ and concatenate the same with sorted list $M_{v}^{(d)}$ to obtain the intended subgraph $s g_{v}^{(d)}$ (line 7).

Example. To illustrate the subgraph extraction process, lets consider the examples in Fig. 6.1. Lets consider the graph 1(c) as the complete graph from which we intend to get the degree 0,1 and 2 subgraph around the root node HttpURL. init. Subjecting these inputs to Algorithm 6, we get subgraphs \{HttpURL. init\}, \{HttpURL . init -> OpenConnection\} and $\{$ HttpURL . init $\rightarrow$ OpenConnection -> Connect $\}$ for degrees 0,1 and 2, respectively. 


\section{Radial Skipgram}

Once the subgraph $s g_{v}^{(d)}$, around the root node $v$ is extracted, Algorithm 5 proceeds to learn its embeddings with the radial skip gram model (line 10). Similar to the vanilla skipgram algorithm which learns the embeddings of a target word from its surrounding linear context in a given document, our approach learns the embeddings of a target subgraph using its surrounding radial context in a given graph. The radial skipgram procedure is presented in Algorithm 7.

Modeling the radial context. The radial context around a target subgraph is obtained using the process explained below. As discussed previously in §6.4.1, natural language text have linear co-occurrence relationships. For instance, skipgram model iterates over all possible collocations of words in a given sentence and in each iteration it considers one word in the sentence as the target word and the words occurring in its context window as context words. This is directly usable on graphs if we model linear substructures such as walks or paths with the view of building node representations. For instance, Deep Walk [101] uses a similar approach to learn a target node's representation by generating random walks around it. However, unlike words in a traditional text corpora, subgraphs do not have a linear co-occurrence relationship. Therefore, we intend to consider the breadth-first neighbours of the root node as its context as it directly follows from the definition of WL relabeling process.

To this end, we define the context of a degree- $d$ subgraph $s g_{v}^{(d)}$ rooted at $v$, as the multiset of subgraphs of degrees $d-1, d$ and $d+1$ rooted at each of the neighbours of $v$ (lines 2-6 in Algorithm 7). Clearly this models a radial context rather than a linear one. Note that we consider subgraphs of degrees $d-1, d$ and $d+1$ to be in the context of a subgraph of degree $d$. This is because, as explained with example earlier in $\S 6.1 .2$, a degree- $d$ subgraph is likely to be rather similar to subgraphs of degrees that are closer to $d$ (e.g., $d-1, d+1$ ) and not just degree- $d$ subgraphs only. 
Vanilla Skip Gram. As explained previously in §6.4.1, the vanilla skipgram language model captures fixed-length linear contexts over the words in a given sentence. However, for learning a subgraph's radial context arrived at line 6 in Algorithm 7, the vanilla skipgram model could not be used. Hence we propose a minor modification to consider a radial context as explained below.

Modification. The embedding of a target subgraph, $s g_{v}^{(d)}$, with context context $t_{v}^{(d)}$ is learnt using lines 7 - 9 in Algorithm 7. Given the current representation of target subgraph $\Phi\left(s g_{v}^{(d)}\right)$, we would like to maximize the probability of every subgraph in its context $s g_{\text {cont }}$ (lines 8 and 9). We can learn such posterior distribution using several choices of classifiers. For example, modeling it using logistic regression would result in a huge number of labels that is equal to $\left|S G_{\text {vocab }}\right|$. This could be in several thousands/millions in the case of large graphs. Training such models would require large amount of computational resources. To alleviate this bottleneck, we approximate the probability distribution using the negative sampling approach.

\section{Negative Sampling}

Given that $s g_{\text {cont }} \in S G_{\text {vocab }}$ and $\left|S G_{\text {vocab }}\right|$ is very large, calculating $\operatorname{Pr}\left(s g_{\text {cont }} \mid \Phi\left(s g_{v}^{(d)}\right)\right)$ in line 8 is prohibitively expensive. Hence we follow the negative sampling strategy (introduced in §6.4.2) to calculate above mentioned posterior probability. In our negative sampling phase for every training cycle of Algorithm 7, we choose a fixed number of subgraphs (denoted as negsamples) as negative samples and update their embeddings as well. Negative samples adhere to the following conditions: if negsamples $=\left\{\right.$ sgneg $_{1}$, sgneg $\left._{2}, \ldots\right\}$, then negsamples $\subset S G_{\text {vocab }}$, |negsamples $|<<| S G_{\text {vocab }} \mid$ and negsamples $\cap$ context $_{v}^{(d)}=\{\}$. This makes $\Phi\left(s g_{v}^{(d)}\right)$ closer to the embeddings of all the subgraphs its context (i.e. $\Phi\left(s g_{\text {cont }}\right) \mid \forall s g_{\text {cont }} \in$ context $\left.t_{v}^{(d)}\right)$ and at the same time distances the same from the embeddings of a fixed number of subgraphs that are not its context (i.e. $\Phi\left(\right.$ sgneg $\left._{i}\right) \mid \forall$ sgneg $_{i} \in$ negsamples). 


\section{Optimization}

Stochastic gradient descent (SGD) optimizer is used to optimize these parameters (line 9, Algorithm 7). The derivatives are estimated using the back-propagation algorithm. The learning rate $\alpha$ is empirically tuned.

\subsubsection{Relation to Deep WL kernel}

As mentioned before, each of the subgraph in $S G_{\text {vocab }}$ is obtained using the WL re-labelling strategy, and hence represents the WL neighbourhood labels of a node. Hence learning latent representations of such subgraphs amounts to learning representations of WL neighbourhood labels. Therefore, once the embeddings of all the subgraph in $S G_{v o c a b}$ are learnt using Algorithm 5, one could use it to build the deep learning variant of the WL kernel among the graphs in $\mathcal{G}$. For instance, we could compute $\mathcal{M}$ matrix such that each entry $\mathcal{M}_{i j}$ computed as $\left\langle\Phi_{i}, \Phi_{j}\right\rangle$ where $\Phi_{i}$ corresponds to learned $\delta$-dimensional embedding of subgraph $i$ (resp. $\Phi_{j}$ ). Thus, matrix $\mathcal{M}$ represents nothing but the pairwise similarities of all the substructures used by the WL kernel. Hence, matrix $\mathcal{M}$ could directly be plugged into eq. (6.2) to arrive at the deep WL kernel across all the graphs in $\mathcal{G}$.

\subsubsection{Use Cases}

Once we compute the subgraph embeddings, they could be used in several practical applications. We list some prominent use cases here:

(1) Graph Classification. Given $\mathcal{G}$, a set of graphs and $Y$, the set of corresponding class labels, graph classification is the task where we learn a model $\mathcal{H}$ such that $\mathcal{H}: \mathcal{G} \rightarrow Y$. To this end, one could feed SubGRAPH2vec's embeddings to a deep learning classifier such as $\mathrm{CNN}$ (as in [102]) to learn $\mathcal{H}$. Alternatively, one could follow a kernel based classification. That is, one could arrive at a deep WL kernel using the subgraph embeddings as discussed 
in §6.5.3, and use kernelized learning algorithm such as SVM to perform classification.

(2) Graph Clustering. Given $\mathcal{G}$, in graph clustering, the task is to group similar graphs together. Here, a graph kernel could be used to calculate the pairwise similarity among graphs in $\mathcal{G}$. Subsequently, relational data clustering algorithms such as AP [108] and Hierarchical Clustering could be used to cluster the graphs.

It is noted that SubGRAPH2vec's use cases are not confined only to the aforementioned tasks. Since Subgraph2vec could be used to learn node representations (i.e., when subgraph of degree 0 are considered, SubGRAPH2VEc provides node embeddings similar to Deep Walk [101] and node2vec [103]). Hence other tasks such as node classification, community detection and link prediction could also performed using SUBGRAPH2VEC's embeddings. However, in our evaluations in this work we consider only graph classification and clustering as they are more prominent.

\subsection{Evaluation}

We evaluate SUBGRAPH2vec's accuracy and efficiency both in supervised and unsupervised learning tasks. Besides experimenting with benchmark datasets, we also evaluate SuBGRAPH2VEC on with real-world program analysis tasks such as malware and code clone detection on large-scale Android malware and clone datasets. Specifically, we intend to address the following RQs: (1) How does SUBGRAPH2vec compare to existing graph kernels for graph classification tasks in terms of accuracy and efficiency on benchmark datasets, (2) How does Subgraph2vec compare to state-of-the-art graph kernels on a real-world unsupervised learning task, namely, code clone detection (3) How does SubGRAPH2vec compare to state-of-the-art graph kernels on a real-world supervised learning task, namely, malware detection.

Evaluation Setup. All the experiments were conducted on a server with $36 \mathrm{CPU}$ cores (Intel E5-2699 2.30GHz processor), NVIDIA GeForce GTX TITAN Black GPU and 200 
Table 6.2: Benchmark dataset statistics

\begin{tabular}{|c|c|c|c|}
\hline Dataset & \# samples & $\begin{array}{l}\text { \# nodes } \\
\text { (avg.) }\end{array}$ & $\begin{array}{c}\text { \# distinct } \\
\text { node labels }\end{array}$ \\
\hline MUTAG & 188 & 17.9 & 7 \\
PTC & 344 & 25.5 & 19 \\
PROTEINS & 1113 & 39.1 & 3 \\
NCI1 & 4110 & 29.8 & 37 \\
NCI109 & 4127 & 29.6 & 38 \\
\hline
\end{tabular}

Table 6.3: Average Accuracy ( \pm std dev.) for SUBGRAPH2VEC and state-of-the-art graph kernels on benchmark graph classification datasets

\begin{tabular}{|l|l|l|l|l|l|}
\hline Dataset & MUTAG & PTC & PROTEINS & NCI1 & NCI109 \\
\hline WL [77] & $80.63 \pm 3.07$ & $56.91 \pm 2.79$ & $72.92 \pm 0.56$ & $80.01 \pm 0.50$ & $80.12 \pm 0.34$ \\
Deep WLYV [85] & $82.95 \pm 1.96$ & $59.04 \pm 1.09$ & $73.30 \pm 0.82$ & $\mathbf{8 0 . 3 1} \pm 0.46$ & $\mathbf{8 0 . 3 2} \pm 0.33$ \\
SUBGRAPH2VEC & $\mathbf{8 7 . 1 7} \pm 1.72$ & $\mathbf{6 0 . 1 1} \pm 1.21$ & $\mathbf{7 3 . 3 8} \pm 1.09$ & $78.05 \pm 1.15$ & $78.39 \pm 1.89$ \\
\hline
\end{tabular}

GB RAM running Ubuntu 14.04.

\subsubsection{Classification on Benchmark Datasets}

Datasets. Five benchmark graph classification datasets namely MUTAG, PTC, PROTEINS, NCI1 and NCI109 are used in this experiment. These datasets belong to chemoand bio-informatics domains and the statistics on the same are reported in Table 6.2. MUTAG dataset consists 188 chemical compounds where class label indicates whether or not the compound has a mutagenic effect on a bacterium. PTC dataset comprises of 344 compounds and the classes indicate carcinogenicity on female/male rats. PROTEINS is a graph collection where nodes are secondary structure elements and edges indicate neighborhood in the amino-acid sequence or in 3D space. NCI1 and NCI109 datasets contain compounds screened for activity against non-small cell lung cancer and ovarian cancer cell lines. Graphs are classified as enzyme or non-enzyme. All these datasets are made available in $[77,85]$.

Comparative Analysis. For classification tasks on each of the datasets, we use the embeddings learnt using SubGRAPH2vec and build the Deep WL kernel as explained in §6.5.3. 
We compare SubGRAPH2VEC against the WL kernel [77] and Yanardag and Vishwanathan's formulation of deep WL kernel [85] (denoted as Deep $\mathrm{WL}_{\mathrm{YV}}$ ).

Configurations. For all the datasets, $90 \%$ of samples are chosen at random for training and the remaining $10 \%$ samples are used for testing. The hyper-parameters of the classifiers are tuned based on 5-fold cross validation on the training set.

Evaluation Metric. The experiment is repeated 5 times and the average accuracy (along with std. dev.) is used to determine the effectiveness of classification. Efficiency is determined in terms of time consumed for learning subgraph embeddings ( $a k a$ pre-training duration).

\section{Results and Discussion.}

Accuracy. Table 6.3 lists the results of the experiments. It is clear that SVMs with SuBGRAPH2VEC's embeddings achieve better accuracy on 3 datasets (MUTAG, PTC and PROTEINS) and comparable accuracy on the remaining 2 datasets (NCI1 and NCI109).

Efficiency. Out of the methods compared, only Deep $\mathrm{WL}_{\mathrm{YV}}$ kernel and SubGraPH2vec involve pre-training to compute vectors of subgraphs. Evidently, pre-training helps them capture latent similarities between the substructures in graphs and thus aids them to outperform traditional graph kernels. Therefore, it is important to study the cost of pre-training. To this end, we report the pre-training durations of these two methods in Fig. 6.2. Being similar in terms of pre-training, both methods require very similar durations to build the pre-trained vectors. However, for the datasets under consideration, SubGRAPH2vec requires lesser time than Deep $\mathrm{WL}_{\mathrm{Yv}}$ kernel as its radial skipgram involves slightly lesser computations than the vanilla skipgram used in Deep $\mathrm{WL}_{\mathrm{Yv}}$ kernel.

However it is important to note that classification on these benchmark datasets are much simpler than real-world classification tasks. In fact, by using trivial features such as number of nodes in the graph, [105] achieved comparable accuracies to the state-of-the-art graph 


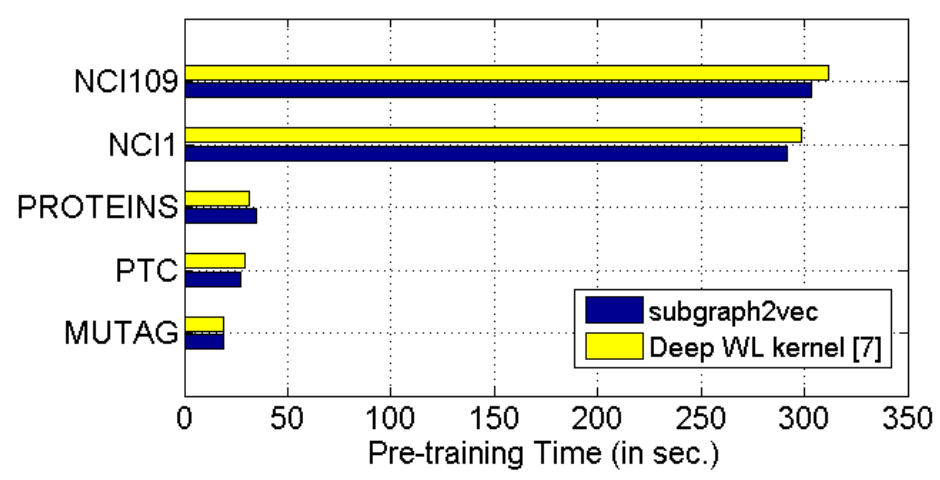

Figure 6.2: Deep WL kernel Vs SubGraPh2vecPre-training Durations.

Table 6.4: Clone Detection Dataset Statistics

\begin{tabular}{|c|c|c|c|c|}
\hline Dataset & \# samples & \# clusters & $\begin{array}{c}\text { \# nodes } \\
\text { (avg.) }\end{array}$ & $\begin{array}{c}\text { \# edges } \\
\text { (avg.) }\end{array}$ \\
\hline CLONE 260 [109] $^{\text {(avg }}$ & 260 & 100 & 9829.15 & 31026.30 \\
\hline
\end{tabular}

kernels. It would be incomplete if we evaluate SUBGRAPH2vec only on these benchmark datasets. Hence in the two subsequent experiments, we involve real-world datasets on practical graph clustering and classification tasks.

\subsubsection{Clone Detection}

Android apps are cloned across different markets by unscrupulous developers for reasons such as stealing advertisement revenue [109]. Detecting and removing such cloned apps is an important task for app market curators that helps maintaining quality of markets and app ecosystem. In this experiment, we consider a set of Android apps and our goal is to cluster them such that clone (semantically similar) apps are grouped together. Hence, this amounts to unsupervised code similarity detection.

Dataset. We acquired a dataset of 260 apps collected from the authors of a recent clone detection work, 3D-CFG [109]. We refer to this dataset as CLONE260. All the apps in CLONE$_{260}$ are manually analyzed and 100 clone sets (i.e. ground truth clusters) are identified by the authors of [109]. The details on this dataset are furnished in Table 6.4. As it could 
Table 6.5: Clone Detection - Results

\begin{tabular}{|c|c|c|c|}
\hline Kernel & WL [77] & Deep WL $L_{Y V}[85]$ & SUBGRAPH2VEC \\
\hline Pre-training duration & & $421.7 \mathrm{~s}$ & $409.28 \mathrm{~s}$ \\
\hline ARI & 0.67 & 0.71 & 0.88 \\
\hline
\end{tabular}

be seen from the table, this problem involves graphs that are much larger/denser than the benchmark datasets used in $§ 6.6 .1$.

Our objective is to reverse engineer these apps, obtain their bytecode and represent the same as graphs. Subsequently, we cluster similar graphs that represent cloned apps together. To achieve this, we begin by representing reverse engineered apps as ICFGs. Nodes of the ICFGs are labeled with Android APIs that they access ${ }^{3}$. Subsequently, we use SUBGRAPH2vEc to learn the vector representations of subgraphs from these ICFGs and build a deep kernel matrix (using eq. (6.2)). Finally, we use AP clustering algorithm [108] over the kernel matrix to obtain clusters of similar ICFGs representing clone apps.

Comparative Analysis. We compare SubGRAPH2vec's accuracy on the clone detection task against the WL [77] and Deep WL $\mathrm{WV}_{\mathrm{Y}}$ [85] kernels.

Evaluation Metric. A standard clustering evaluation metric, namely, Adjusted Rand Index (ARI) is used to determine clone detection accuracy. The ARI values lies in the range $[-1,1]$. A higher ARI means a higher correspondence to ground-truth clone sets.

\section{Results and Discussion.}

Accuracy. The results of clone detection using the three kernels under discussion are presented in Table 6.5. Following observations are drawn from the table:

- Subgraph2vec outperform WL and Deep WLyv kernels by more than $21 \%$ and $17 \%$, respectively. The difference between using Deep WL kernel and SubGRAPH2vec embeddings is more pronounced in the unsupervised learning task.

- WL kernel perform poorly in clone detection task as it, by design, fails to identify the ${ }^{3}$ For more details on app representations, we refer to [28]. 
Table 6.6: Malware Detection Dataset Statistics

\begin{tabular}{|c|c|c|c|c|c|}
\hline Dataset & Class & Source & \# apps & $\begin{array}{c}\text { \# nodes } \\
\text { (avg.) }\end{array}$ & $\begin{array}{c}\text { \# edges } \\
\text { (avg.) }\end{array}$ \\
\hline \multirow{2}{*}{ TraIN $_{10 K}$} & Malware & DrebIN [25] & 5600 & 9590.23 & 19377.96 \\
& Benign & Google Play [4] & 5000 & 20873.71 & 38081.24 \\
\hline \multirow{2}{*}{ TeST $_{10 K}$} & Malware & Virus Share [14] & 5000 & 13082.40 & 25661.93 \\
& Benign & Google Play [4] & 5000 & 27032.03 & 42855.41 \\
\hline
\end{tabular}

subgraph similarities, which is essential to precisely captures the latent program semantics. On the other hand, Deep $\mathrm{WL}_{\mathrm{Yv}}$ kernel performs reasonable well as it captures similarities among subgraphs of same degree. However, it fails to capture the complete semantics of the program due to its strong assumptions (see §6.1.3). Whereas, SubGRAPH2VEC was able to precisely capture subgraph similarities spanning across multiple degrees.

Efficiency. From Table 6.5, it can be seen that the pretraining duration for SUBGRAPH2VEC is slightly better than Deep $\mathrm{WL}_{\mathrm{Yv}}$ kernel. This observation is inline with the pretraining durations of benchmark datasets. WL kernel involves no pre-training and deep kernel computation and hence much more efficient than the other two methods.

\subsubsection{Malware Detection}

Malware detection is a challenging task in the field of cyber-security as the attackers continuously enhance the sophistication of malware to evade novel detection techniques. In the case of Android platform, many existing works such as [28], represent benign and malware apps as ICFGS and cast malware detection as a graph classification problem. Similar to clone detection, this task typically involves large graphs as well.

Datasets. DreBIN [25] provides a collection of 5,560 Android malware apps collected from 2010 to 2012. We collected 5000 benign top-selling apps from Google Play [4] that were released around the same time and use them along with the DreBIN apps to train the malware detection model. We refer to this dataset as $\operatorname{Train}_{10 K}$. To evaluate the performance of 
Table 6.7: Malware Detection - Results

\begin{tabular}{|c|c|c|c|}
\hline Classifier & WL [77] & Deep WL $L_{Y V}[85]$ & SUBGRAPH2VEC \\
\hline Pre-training dur & & $2631.17 \mathrm{~s}$ & $2219.28 \mathrm{~s}$ \\
\hline Accuracy & 66.15 & 71.03 & 74.48 \\
\hline
\end{tabular}

the model, we use a more recent set of 5000 malware samples (i.e., collected from 2010 to 2014) provided by Virus share [14] and an equal number of benign apps from Google Play that were released around the same time. We refer to this dataset as Test ${ }_{10 K}$. Hence, in total, our malware detection experiments involve 20,600 apps. The statistics of this dataset is presented in Table 6.6.

Comparative Analysis and Evaluation Metrics. The same type of comparative analysis and evaluation metrics against WL and Deep WLYv kernels used in experiments with benchmark datasets in $\S 6.6 .1$ are used here as well.

\section{Results \& Discussion.}

Accuracy. The results of malware detection using the three kernels under discussion are presented in Table 6.7. Following observations are drawn from the table:

- SVM built using Subgraph2vec embeddings outperform WL and Deep WLYv kernels by more than $12 \%$ and $4 \%$, respectively. This improvement could be attributed to SuBGRAPH2VEC's high quality embeddings learnt from apps' ICFGs.

- On this classification task, both Deep $\mathrm{WL}_{\mathrm{YV}}$ and SuBgRAPH2vec outperform WL kernel by a significant margin (unlike the experiments on benchmark datasets). Clearly, this is due to the fact that the former methods capture the latent subgraph similarities from ICFGs which helps them learn semantically similar but syntactically different malware features.

Efficiency. The inferences on pre-training efficiency discussed in $§ 6.6 .1$ and $\$ 6.6 .2$ hold for this experiment as well. 


\subsection{Conclusion}

In this chapter, we presented SubGRAPH2vec, an unsupervised representation learning technique to learn embedding of rooted subgraphs that exist in large graphs. Through our large-scale experiments involving benchmark and real-world graph classification and clustering datasets, we demonstrate that subgraph embeddings learnt by our approach could be used in conjunction with classifiers such as CNNs, SVMs and relational data clustering algorithms to achieve significantly superior accuracies. On real-world application involving large graphs, SubGRaPh2vec outperforms state-of-the-art graph kernels significantly without compromising efficiency of the overall performance. We make all the code and data used within this work available at: https://sites.google.com/site/subgraph2vec 


\section{Chapter 7}

\section{Conclusion and Future Research}

In this chapter, the research work that we have conducted in the thesis are summarized and our future research directions and works are discussed.

\subsection{Summary of Completed Work}

Along with the raising prominence of Android system, both its users and developers are facing critical threats from Android ecosystem in the form of malware and repackaged clone apps. In this thesis, we conducted a series of works to develop suitable representations of apps to perform large-scale semantic detection of malware and clone apps.

First, we observe that apps' PRGs are ideally suited for malware detection as they are robust against several transformation and evasion attacks. To embed these PRGs towards catering accurate and scalable malware detection, we propose a graph kernel named CWLK. Unlike the existing kernels which capture only the security-sensitive neighborhoods in PRGs, CWLK captures these neighborhoods along with the context under which they are reachable. This makes CWLK more expressive and in turn more accurate than existing kernels.

Second, we propose a framework, named CASANDRA, to perform context-aware, adap- 
tive, scalable and explainable Android malware detection. The main goals of CASANDRA are to automatically adapt to the drift malware population and to provide explanations of its predictions. To this end, the framework uses CWLK for representation and a linear online classifier for explainable and self-adaptive learning. Large-scale evaluations demonstrated that CASANDRa could adapt to evolution in real-world malware over time helping it to outperform existing techniques (that are not self-adaptive) significantly in terms of accuracy while maintaining comparable efficiency.

Third, we observe that multiple sets of features such as API dependencies, CFG structures, information sources/sinks could be extracted from PRGs each representing a distinct semantic views of the app. Capturing multiple such views would lead to a more comprehensive and accurate detection. To this end, we proposed a framework named MKLDROID that uses CWLK for PRG representation and MKL for multi-view learning. Besides comprehensive detection, a salient feature of MKLDRoID is its ability to locate malicious code portions in PRGs. Through several large-scale experiments, we demonstrated the framework's effective multi-view context-aware malware detection and malicious code localization potentials.

Fourth, we observe that when using PRGs with existing graph kernels such as WLK to perform malware or clone detection, they are inherently incapable of appropriately identifying the similarities of syntactically different but semantically equivalent portions of code in PRG subgraphs. To address this limitation, we proposed an unsupervised subgraph embedding learning technique named SuBGRAPH2vec and leveraged on it develop a deep learning variant of WLK. Our experiments revealed that Deep WLK could improve the accuracies of WLK on both clone and malware detection tasks. 


\subsection{Future Work}

In this thesis, we have developed techniques to represent PRGs facilitating semantic detection of malware and clone apps and also addressed some of the problem-specific challenges such as handling malware population drift and malicious code localization. However, due to the immensity of Android malware and repackaged clones, there are still many security issues that are not covered in this thesis. In future, we are going to conduct the following works:

- Deep CWLK and contextual variants of other existing kernels. WLK, as discussed earlier, suffers with limitations such as diagonal dominance and inability to evaluate semantic similarities between syntactically different PRG subgraphs. Addressing these, we developed a deep learning variant of WLK. However, CWLK which is a context-aware version of WLK still suffers from the two above mentioned limitations. Hence, an immediate and natural extension would be to develop a context-aware version of SUBgRAPH2vec and thereby deep CWLK. Also, on similar lines of future work, we plan to investigate incorporating contextual information in other sub-structure based graph kernels such as NHGK [78] and NSPDK [79] and subsequently, study their suitability for performing malware detection.

- Online MKL for adaptive and comprehensive malware detection. The strength of our CASANDRA framework lies in its use of online learning towards being selfadaptive to malware population drift and the strength of our MKLDRoID framework lies in its use of MKL to facilitate comprehensive multi-view detection. However, integrating these two learning paradigms towards offering a comprehensive and selfadaptive malware detection thereby combining the best from both the worlds remains as a future work. Specifically, we intend to explore using Online MKL algorithms such as Fourier Online Gradient Descent and Nystrom Online Gradient Descent [93] 
to achieve this. Towards incorporating more complementary semantic view, we intend to include data-flow analysis and dynamic analysis to our framework. Furthermore, on similar lines of research, we intend to explore other incremental learning paradigms such as progressive learning [120] to handle specific problems such as malware familial classification where the number of target classes keeps monotonically increasing.

- Cross-platform malware detection. As noted earlier, Android apps are which written predominantly in Java are much easier to be disassembled or reverse engineered for various unscrupulous activities. However, iOS apps which are written in objective $\mathrm{C}$ do not pose such ease of reversing engineering. Therefore, it is easier to analyze Android apps and detect malware and leverage this knowledge to detect iOS malware. To this end, we intend to study the relevance between Android and iOS platforms and use this knowledge for performing cross-platform malware detection. More specifically, we first use SubgraPh2VEc embedding with Machine Translation reproaches such as RNNs (e.g., encoder-decoder model [121]) to map Android APIs to similar iOS APIs. Subsequently, we leverage on this mapping and use API sequences as features to detect iOS malware that are semantically similar to existing Android ones.

- Other applications of code similarity search. We have developed two PRG representation techniques in this thesis, namely, CWLK and SubGraph2vec. Out of these CWLK is task-specific i.e., it could not be used for anything except malware detection. However, Subgraph2vec is a general purpose graph kernel and is highly effective for semantic comparison of arbitrary graphs, including PRGs. Hence, this makes SUBGRAPH2VEC a general software similarity detection technique and this opens up a plethora of potential applications which leverage on software similarity search such as vulnerability detection [126-128], app tagging [129], detecting third-party 
libraries [131, 132], recommending similar apps [130] and many more. We intend to focus on these applications of SUBGRAPH2vec in the future.

Finally, as a part of our ongoing extension of the works in this thesis, we are in the process of preparing a manuscript on the idea discussed below.

Asum: A Deep Learning based Framework for Malware Attack Summarization and Familial Analysis.

Existing ML based techniques have used semantic features such as system calls, APIs, control- and data-flow sequences to perform malware familial classification. Though these features facilitate capturing common malice behaviors, they are incapable of modeling syntactically different yet semantically similar behaviors. This capability is of paramount importance to malware familial profiling and identifying similar attacks across different generations of malware, different malware families and even different platforms. We refer to these semantically similar malice behaviors as attack summaries and the process of extracting them as attack summarization. To address the above-said limitation and perform effective attack summarization, we intend to use SUBGRAPH2VEC embeddings from PRGs as they demonstrate excellent capabilities to capture semantic similarities. In this work, we propose a three-stage framework named Asum that uses deep learning, classification and clustering techniques for summarizing attacks to perform malware familial classification. In the first stage, Asum uses Subgraph2vec to learn PRG subgraph embeddings such that semantically similar subgraphs have similar representations. In the second stage, we propose an approach for extracting attack summaries from subgraphs representations through a combined use of clustering and graph kernel techniques. In the third stage, Asum builds a profile for each malware family which is nothing but a vector of attack summaries. Subsequently, with these attack summary vectors, we use kernel classifiers to perform malware familial classification. 


\section{Author's Publications}

The publications that are not included in the thesis are marked with an asterisk.

\section{Accepted Journals}

1. Narayanan, A., Mahinthan, C., Chen, L., \& Liu, Y., Context-aware, Adaptive and Scalable Android Malware Detection through Online Learning. IEEE Transactions on Emerging Topics in Computational Intelligence 1, no. 3 (2017): 157-175.

2. Narayanan, A., Mahinthan, C., Chen, L., \& Liu, Y., A Multi-view Context-aware Approach to Android Malware Detection and Malicious Code Localization (accepted in Springer Empirical Software Engineering Journal).

\section{Accepted Conference/Workshop Papers}

1. Narayanan, A., Chandramohan, M., Chen, L., Liu, Y., \& Saminathan, S. "subgraph2vec: Learning distributed representations of rooted sub-graphs from large graphs". Mining and Learning with Graphs (MLG), KDD Workshop on. ACM, 2016.

2. Narayanan, A., Meng, G., Yang, L., Liu, J., \& Chen, L. (2016, July). Contextual Weisfeiler-Lehman Graph Kernel for Malware Detection. In Neural Networks (IJCNN), 2016 International Joint Conference on (pp. 4701-4708). IEEE. 
3. Narayanan, A., Yang, L., Chen, L., \& Jinliang, L. (2016, July). Adaptive and scalable Android Malware Detection through Online Learning. In Neural Networks (IJCNN), 2016 International Joint Conference on (pp. 2484-2491). IEEE.

4. * Meng, G., Xue, Y., Mahinthan, C., Narayanan, A., Liu, Y., Zhang, J., \& Chen, T. (2016, May). Mystique: Evolving Android Malware for Auditing Anti-Malware Tools. In Proceedings of the 11th ACM on Asia Conference on Computer and Communications Security (pp. 365-376). ACM. Chicago

5. * Meng, G., Xue, Y., Xu, Z., Liu, Y., Zhang, J., \& Narayanan, A. (2016, July). Semantic modelling of Android malware for effective malware comprehension, detection, and classification. In Proceedings of the 25th International Symposium on Software Testing and Analysis (pp. 306-317). ACM.

6. * Narayanan, A., Chen, L., \& Chan, C. K. (2014, April). Addetect: Automated Detection of Android Ad Libraries using Semantic Analysis. In Intelligent Sensors, Sensor Networks and Information Processing (ISSNIP), 2014 IEEE Ninth International Conference on (pp. 1-6). IEEE.

\section{Under Preparation}

1. Narayanan, A., Chandramohan, M., Chen, L., \& Liu, Y., A Asum: Deep Learning based Framework for Malware Attack Summarization and Familial Analysis. — To be submitted to IEEE Trans. on Information Forensics and Security. 


\section{References}

[1] Kaspersky 2016 Threat Report. https://kasperskycontenthub.com/ securelist/files/2016/12/Kaspersky_Security_Bulletin_2016_Review_ ENG.pdf

[2] Symantec 2016 Threat Report. https://www.symantec.com/content/dam/ symantec/docs/reports/istr-21-2016-en.pdf

[3] Smartphone OS Market Share, 2016 Q2. http://www.idc.com/prodserv/ smartphone-os-market-share.jsp

[4] Google Play. https://play.google.com/store

[5] URL https://www. amazon.com/mobile-apps/b?ie=UTF8\&node=2350149011

[6] Anzhi third-party market. www.anzhi.com

[7] AppChina third-party market. www. appchina.com

[8] SlideMe third-party market. www.SlideME.org

[9] HiApk third-party market. www. hiapk.com

[10] FDroid third-party market. www. fdroid.org

[11] Angeeks third-party market. http://apk. angeeks.com

[12] AppChina third-party market. www. appchina.com

[13] Android Drawer third-party market. https: //www. androiddrawer.com

[14] Virus Share malware dataset: http://virusshare.com

[15] Virus Total web portal. https://www.virustotal.com/ 
[16] Androguard. https://code.google.com/p/androguard

[17] Soot framework. http://sable.github.io/soot

[18] POUIK, AND G0RFI3LD. Similarities for fun \& profit. Phrack 14, 68 (April 2012). http: //www. phrack.org/issues.html?id=15\&issue=68

[19] Adagio source code. https://github.com/hgascon/adagio

[20] McAfee, McAfee Labs Threats Report, Tech. Rep., Mar 2016.

[21] Cesare, S., \& Xiang, Y. (2010, January). Classification of malware using structured control flow. In Proceedings of the Eighth Australasian Symposium on Parallel and Distributed Computing-Volume 107 (pp. 61-70). Australian Computer Society, Inc.

[22] Guyon, I., \& Elisseeff, A. (2003). An introduction to variable and feature selection. The Journal of Machine Learning Research, 3, 1157-1182.

[23] Tam, Kimberly, et al. "The Evolution of Android Malware and Android Analysis Techniques.” ACM Computing Surveys (CSUR) 49.4 (2017): 76.

[24] Zhou, Yajin, and Xuxian Jiang. "Dissecting android malware: Characterization and evolution.” Security and Privacy (SP), 2012 IEEE Symposium on. IEEE, 2012.

[25] Arp, Daniel, et al. "Drebin: Effective and explainable detection of android malware in your pocket." Proceedings of the Annual Symposium on Network and Distributed System Security (NDSS). 2014.

[26] Allix, Kevin, et al. "Empirical assessment of machine learning-based malware detectors for Android." Empirical Software Engineering (2014): 1-29.

[27] Allix, Kevin, et al. "Machine Learning-Based Malware Detection for Android Applications: History Matters!", TR-2014

[28] Yang, Wei, et al. "Appcontext: Differentiating malicious and benign mobile app behaviors using context." Software Engineering (ICSE), 2015 IEEE/ACM 37th IEEE International Conference on. Vol. 1. IEEE, 2015.

[29] Zhang, Mu, et al. "Semantics-aware Android malware classification using weighted contextual API dependency graphs." Proceedings of the 2014 ACM SIGSAC Conference on Computer and Communications Security. ACM, 2014. 
[30] Elish, Karim O., et al. "Profiling user-trigger dependence for Android malware detection." Computers \& Security 49 (2015): 255-273.

[31] Yang, Chao, et al. "Droidminer: Automated mining and characterization of finegrained malicious behaviors in android applications." Computer Security-ESORICS 2014. Springer International Publishing, 2014. 163-182.

[32] Gascon, Hugo, et al. "Structural detection of android malware using embedded call graphs." Proceedings of the 2013 ACM workshop on Artificial intelligence and security. ACM, 2013.

[33] Mariconti, Enrico, et al. "MAMADROID: Detecting Android Malware by Building Markov Chains of Behavioral Models." arXiv preprint arXiv:1612.04433 (2016).

[34] Chen, Kai, et al. "Finding unknown malice in 10 seconds: Mass vetting for new threats at the google-play scale." 24th USENIX Security Symposium (USENIX Security 15). 2015.

[35] Peng, Wei, et al. "ACTS: Extracting Android App Topological Signature through Graphlet Sampling."

[36] Martinelli, Fabio, et al. "Classifying android malware through subgraph mining." Data Privacy Management and Autonomous Spontaneous Security. Springer Berlin Heidelberg, 2014. 268-283.

[37] Meng, Guozhu, et al. "Semantic modelling of Android malware for effective malware comprehension, detection, and classification." Proceedings of the 25th International Symposium on Software Testing and Analysis. ACM, 2016.

[38] Sahs, Justin, and Latifur Khan. "A machine learning approach to android malware detection.” Intelligence and Security Informatics Conference (EISIC), 2012 European. IEEE, 2012.

[39] Chakradeo, Saurabh, et al. "Mast: triage for market-scale mobile malware analysis." Proceedings of the sixth ACM conference on Security and privacy in wireless and mobile networks. ACM, 2013.

[40] Saracino, Andrea, et al. "Madam: Effective and efficient behavior-based android malware detection and prevention." IEEE Transactions on Dependable and Secure Computing (2016). 
[41] Aafer, Yousra et al. "DroidAPIMiner: Mining API-level features for robust malware detection in android." International Conference on Security and Privacy in Communication Systems. Springer International Publishing, 2013.

[42] Peiravian, Naser, and Xingquan Zhu. "Machine learning for android malware detection using permission and api calls." 2013 IEEE 25th International Conference on Tools with Artificial Intelligence. IEEE, 2013.

[43] Gorla, Alessandra, et al. "Checking app behavior against app descriptions." Proceedings of the 36th International Conference on Software Engineering. ACM, 2014.

[44] Lindorfer, Martina, et al. "Marvin: Efficient and comprehensive mobile app classification through static and dynamic analysis." Computer Software and Applications Conference (COMPSAC), 2015 IEEE 39th Annual. Vol. 2. IEEE, 2015.

[45] Zhauniarovich, Yury, et al. "Stadyna: Addressing the problem of dynamic code updates in the security analysis of android applications." Proceedings of the 5th ACM Conference on Data and Application Security and Privacy. ACM, 2015.

[46] Xu, Lifan, et al. "HADM: Hybrid Analysis for Detection of Malware."

[47] Chen, Sen, et al. "StormDroid: A Streaminglized Machine Learning-Based System for Detecting Android Malware." Proceedings of the 11th ACM on Asia Conference on Computer and Communications Security. ACM, 2016.

[48] Dash, Santanu Kumar, et al. "DroidScribe: Classifying Android Malware Based on Runtime Behavior.” Mobile Security Technologies (MoST 2016) 7148 (2016): 1-12.

[49] Suarez-Tangil, Guillermo, et al. "DroidSieve: Fast and Accurate Classification of Obfuscated Android Malware." (2016).

[50] Deo, Amit, et al. "Prescience: Probabilistic Guidance on the Retraining Conundrum for Malware Detection" Proceedings of the 2016 ACM workshop on Artificial intelligence and security. ACM, 2016.

[51] Au, Kathy Wain Yee, et al. "Pscout: analyzing the android permission specification." Proceedings of the 2012 ACM conference on Computer and communications security. ACM, 2012. 
[52] Arzt, Steven, et al. "Susi: A tool for the fully automated classification and categorization of android sources and sinks." In 2014 Network and Distributed System Security Symposium (NDSS).

[53] Arzt, Steven, et al. "Flowdroid: Precise context, flow, field, object-sensitive and lifecycle-aware taint analysis for android apps.” Acm Sigplan Notices 49.6 (2014): 259-269.

[54] Li, Li, et al. "Iccta: Detecting inter-component privacy leaks in android apps." Proceedings of the 37th International Conference on Software Engineering-Volume 1. IEEE Press, 2015.

[55] Gordon, Michael I., et al. "Information Flow Analysis of Android Applications in DroidSafe." NDSS. 2015.

[56] Octeau, Damien, et al. "Effective inter-component communication mapping in android: An essential step towards holistic security analysis." Presented as part of the 22nd USENIX Security Symposium (USENIX Security 13). 2013.

[57] Octeau, Damien, et al. "Composite constant propagation: Application to android inter-component communication analysis." Proceedings of the 37th International Conference on Software Engineering-Volume 1. IEEE Press, 2015.

[58] Avdiienko, Vitalii, et al. "Mining apps for abnormal usage of sensitive data." Software Engineering (ICSE), 2015 IEEE/ACM 37th IEEE International Conference on. Vol. 1. IEEE, 2015.

[59] Garcia, Joshua, et al. "Obfuscation-resilient, efficient, and accurate detection and family identification of android malware.” Department of Computer Science, George Mason University, Tech. Rep (2015).

[60] Burguera, Iker, et al. "Crowdroid: behavior-based malware detection system for android." Proceedings of the 1st ACM workshop on Security and privacy in smartphones and mobile devices. ACM, 2011.

[61] Tripp, Omer, and Julia Rubin. "A Bayesian Approach to Privacy Enforcement in Smartphones." USENIX Security. Vol. 14. 2014.

[62] Enck, William, et al. "TaintDroid: an information-flow tracking system for realtime privacy monitoring on smartphones." ACM Transactions on Computer Systems (TOCS) 32.2 (2014): 5. 
[63] Fredrikson, Matt, et al. "Synthesizing near-optimal malware specifications from suspicious behaviors." Security and Privacy (SP), 2010 IEEE Symposium on. IEEE, 2010.

[64] Anderson, Blake, et al. "Graph-based malware detection using dynamic analysis." Journal in Computer Virology 7.4 (2011): 247-258.

[65] Singh, Anshuman, et al. "Tracking concept drift in malware families." Proceedings of the 5th ACM workshop on Security and artificial intelligence. ACM, 2012.

[66] Kantchelian, Alex, et al. "Approaches to adversarial drift." Proceedings of the 2013 ACM workshop on Artificial intelligence and security. ACM, 2013.

[67] Ma, Justin, et al. "Identifying suspicious URLs: an application of large-scale online learning." Proceedings of the 26th Annual International Conference on Machine Learning. ACM, 2009.

[68] Roy, Sankardas, et al. "Experimental study with real-world data for android app security analysis using machine learning." Proceedings of the 31st Annual Computer Security Applications Conference. ACM, 2015.

[69] Allix, Kevin, et al. "Androzoo: Collecting millions of android apps for the research community." Proceedings of the 13th International Conference on Mining Software Repositories. ACM, 2016.

[70] Li, Li, et al. "Understanding Android App Piggybacking: A Systematic Study of Malicious Code Grafting.” IEEE Transactions on Information Forensics and Security (2017).

[71] Li, Li, et al. "Automatically Locating Malicious Packages in Piggybacked Android Apps." Proceedings of the International Workshop on Mobile Software Engineering and Systems. ACM, 2017.

[72] Tian, Ke, et al. "Analysis of Code Heterogeneity for High-Precision Classification of Repackaged Malware.” Security and Privacy Workshops (SPW), 2016 IEEE. IEEE, 2016.

[73] Kriege, Nils M., et al. "A Unifying View of Explicit and Implicit Feature Maps for Structured Data: Systematic Studies of Graph Kernels." arXiv preprint arXiv:1703.00676 (2017). 
[74] Gärtner, Thomas, et al. "On graph kernels: Hardness results and efficient alternatives." Learning Theory and Kernel Machines. Springer Berlin Heidelberg, 2003. 129-143.

[75] Kang, U., et al. "Fast random walk graph kernel." Proceedings of the 2012 SIAM International Conference on Data Mining. Society for Industrial and Applied Mathematics, 2012.

[76] Borgwardt, Karsten M., and Hans-Peter Kriegel. "Shortest-path kernels on graphs." Fifth IEEE International Conference on Data Mining (ICDM'05). IEEE, 2005.

[77] Shervashidze, Nino, et al. "Weisfeiler-lehman graph kernels." The Journal of Machine Learning Research 12 (2011): 2539-2561.

[78] Hido, Shohei, and Hisashi Kashima. "A linear-time graph kernel." Data Mining, 2009. ICDM'09. Ninth IEEE International Conference on. IEEE, 2009.

[79] Costa, Fabrizio, and Kurt De Grave. "Fast neighborhood subgraph pairwise distance kernel." Proceedings of the 26th International Conference on Machine Learning. Omnipress, 2010.

[80] Navarin, N., Sperduti, A., \& Tesselli, R. (2015, November). Extending local features with contextual information in graph kernels. In Neural Information Processing (pp. 271-279). Springer International Publishing.

[81] Fröhlich, Holger, Jörg K. Wegner, and Andreas Zell. ”Assignment kernels for chemical compounds." Neural Networks, 2005. IJCNN'05. Proceedings. 2005 IEEE International Joint Conference on. Vol. 2. IEEE, 2005.

[82] Mahé, Pierre, et al. "Extensions of marginalized graph kernels." Proceedings of the twenty-first international conference on Machine learning. ACM, 2004.

[83] Borgwardt, Karsten M., et al. "Protein function prediction via graph kernels.” Bioinformatics 21.suppl 1 (2005): i47-i56.

[84] Harchaoui, Zäid, and Francis Bach. "Image classification with segmentation graph kernels." Computer Vision and Pattern Recognition, 2007. CVPR'07. IEEE Conference on. IEEE, 2007.

[85] P. Yanardag and S. Vishwanathan. "Deep graph kernels." In Proc. of SIGKDD, 2015. 
[86] Friedman, Jerome, et al. "The elements of statistical learning." Vol. 1. Springer, Berlin: Springer series in statistics, 2001.

[87] Pedregosa, Fabian, et al. "Scikit-learn: Machine learning in Python." Journal of Machine Learning Research 12.Oct (2011): 2825-2830.

[88] Ribeiro, M. T., et al. "Why Should I Trust You?”: Explaining the Predictions of Any Classifier. In Proc. of SIGKDD, 2016.

[89] Harrold, M. J., et al. "Computation of interprocedural control dependence.” In ACM SIGSOFT Software Engineering Notes (Vol. 23, No. 2, pp. 11-20).

[90] Biggio, Battista, et al. "Poisoning behavioral malware clustering." Proceedings of the 2014 Workshop on Artificial Intelligent and Security Workshop. ACM, 2014.

[91] Gönen, Mehmet, and Ethem Alpaydın. "Multiple kernel learning algorithms.” Journal of Machine Learning Research 12.Jul (2011): 2211-2268.

[92] Sun, Z., Ampornpunt, N., Varma, M., \& Vishwanathan, S. (2010). Multiple kernel learning and the SMO algorithm. In Advances in neural information processing systems (pp. 2361-2369).

[93] Lu, Jing, et al. ”Large scale online kernel learning.” J. Mach. Learn. Res (2015).

[94] Forman, George. "An extensive empirical study of feature selection metrics for text classification.” Journal of machine learning research 3.Mar (2003): 1289-1305.

[95] Burges, Christopher JC. "A tutorial on support vector machines for pattern recognition.” Data mining and knowledge discovery 2.2 (1998): 121-167.

[96] Meng, Guozhu, et al. "Mystique: Evolving Android Malware for Auditing AntiMalware Tools." Proceedings of the 11th ACM on Asia Conference on Computer and Communications Security. ACM, 2016.

[97] Hassen, Mehadi, and Philip K. Chan. "Scalable Function Call Graph-based Malware Classification."

[98] Searles, Robert, et al. "Parallelization of Machine Learning Applied to Call Graphs of Binaries for Malware Detection." Proceedings of the 25th Euromicro International Conference on Parallel, Distributed and Network-Based Processing, PDP 2017, St. Petersburg, Russia, 2017. 
[99] Vishwanathan, S. V. N., et al. "Graph kernels." The Journal of Machine Learning Research 11 (2010): 1201-1242.

[100] Shervashidze, Nino, et al. "Efficient graphlet kernels for large graph comparison." International conference on artificial intelligence and statistics. 2009.

[101] Perozzi, Bryan, et al. "Deepwalk: Online learning of social representations." Proceedings of the 20th ACM SIGKDD international conference on Knowledge discovery and data mining. ACM, 2014.

[102] Niepert, Mathias, et al. "Learning Convolutional Neural Networks for Graphs." Proceedings of the 33rd annual international conference on machine learning. ACM, 2016.

[103] Grover, Aditya, \& Leskovec, Jure. "node2vec: Scalable Feature Learning for Networks." Proceedings of the 22nd ACM SIGKDD International Conference on Knowledge Discovery and Data Mining. ACM, 2016.

[104] Horváth, Tamás, et al. Cyclic pattern kernels for predictive graph mining. In Proceedings of the International Conference on Knowledge Discovery and Data Mining (KDD), pages 158-167, 2004.

[105] Orlova, Yuliia, et al. "Graph kernel benchmark data sets are trivial!" ICML Workshop on Features and Structures FEATS 2015.

[106] Yanardag, Pinar, and S. V. N. Vishwanathan. "A Structural Smoothing Framework For Robust Graph Comparison.” Advances in Neural Information Processing Systems. 2015.

[107] Mikolov, Tomas, et al. "Efficient estimation of word representations in vector space." ICLR Workshop, 2013.

[108] Frey, Brendan J., and Delbert Dueck. "Clustering by passing messages between data points." science 315.5814 (2007): 972-976.

[109] Chen, Kai, et al. "Achieving accuracy and scalability simultaneously in detecting application clones on android markets." Proceedings of the 36th International Conference on Software Engineering. ACM, 2014. 
[110] Wang, Haoyu, et al. "Wukong: a scalable and accurate two-phase approach to android app clone detection." Proceedings of the 2015 International Symposium on Software Testing and Analysis. ACM, 2015.

[111] Zhou, Wu, et al. "Fast, scalable detection of piggybacked mobile applications." Proceedings of the third ACM conference on Data and application security and privacy. ACM, 2013.

[112] Blum, Avrim. On-line algorithms in machine learning. Springer Berlin Heidelberg, 1998.

[113] Ma, Justin, et al. "Identifying suspicious URLs: an application of large-scale online learning." Proceedings of the 26th Annual International Conference on Machine Learning. ACM, 2009.

[114] Rosenblatt, Frank. "The perceptron: a probabilistic model for information storage and organization in the brain." Psychological review 65.6 (1958): 386.

[115] Zhang, Tong. "Solving large scale linear prediction problems using stochastic gradient descent algorithms." Proceedings of the twenty-first international conference on Machine learning. ACM, 2004.

[116] Crammer, Koby, et al. "Online passive-aggressive algorithms." The Journal of Machine Learning Research 7 (2006): 551-585.

[117] Dredze, Mark, et al. "Confidence-weighted linear classification." Proceedings of the 25th international conference on Machine learning. ACM, 2008.

[118] Dekel, Ofer, et al. "The Forgetron: A kernel-based perceptron on a fixed budget." Advances in neural information processing systems. 2005.

[119] Orabona, Francesco, et al. "The projectron: a bounded kernel-based perceptron." Proceedings of the 25th international conference on Machine learning. ACM, 2008.

[120] Venkatesan, Rajasekar, and Meng Joo Er. "A novel progressive learning technique for multi-class classification.” Neurocomputing 207 (2016): 310-321.

[121] Cho, Kyunghyun, et al. "Learning phrase representations using RNN encoderdecoder for statistical machine translation." arXiv preprint arXiv:1406.1078 (2014). 
[122] Li, Wenchao, et al. "Detecting similar programs via the Weisfeiler-Leman graph kernel.” International Conference on Software Reuse. Springer International Publishing, 2016 .

[123] Bengio, Yoshua, et al. "Representation learning: A review and new perspectives." IEEE transactions on pattern analysis and machine intelligence 35.8 (2013): 17981828.

[124] Socher, Richard, et al. "Deep learning for NLP." Tutorial at Association of Computational Logistics (ACL), 2012, and North American Chapter of the Association of Computational Linguistics (NAACL) (2013).

[125] Perozzi, Bryan, Vivek Kulkarni, and Steven Skiena. "Walklets: Multiscale Graph Embeddings for Interpretable Network Classification." arXiv preprint arXiv:1605.02115 (2016).

[126] Watanabe, Takuya, et al. "A Study on the Vulnerabilities of Mobiles Apps associated with Software Modules.” arXiv preprint arXiv:1702.03112 (2017).

[127] Lu, Long, et al. "Chex: statically vetting android apps for component hijacking vulnerabilities." Proceedings of the 2012 ACM conference on Computer and communications security. ACM, 2012.

[128] Sounthiraraj, David, et al. "Smv-hunter: Large scale, automated detection of ssl/tls man-in-the-middle vulnerabilities in android apps." In Proceedings of the 21st Annual Network and Distributed System Security Symposium (NDSS'14. 2014.

[129] Chen, Ning, et al. "Mobile app tagging." Proceedings of the Ninth ACM International Conference on Web Search and Data Mining. ACM, 2016.

[130] Chen, Ning, et al. "SimApp: A framework for detecting similar mobile applications by online kernel learning." Proceedings of the Eighth ACM International Conference on Web Search and Data Mining. ACM, 2015.

[131] Backes, Michael, et al. "Reliable third-party library detection in android and its security applications." Proceedings of the 2016 ACM SIGSAC Conference on Computer and Communications Security. ACM, 2016.

[132] Ma, Ziang, et al. "Libradar: Fast and accurate detection of third-party libraries in android apps." Proceedings of the 38th International Conference on Software Engineering Companion. ACM, 2016. 
[133] Shawe-Taylor, John, and Nello Cristianini. Kernel methods for pattern analysis. Cambridge university press, 2004.

[134] Wilcoxon, Frank. "Individual comparisons by ranking methods." Biometrics bulletin 1.6 (1945): 80-83. 